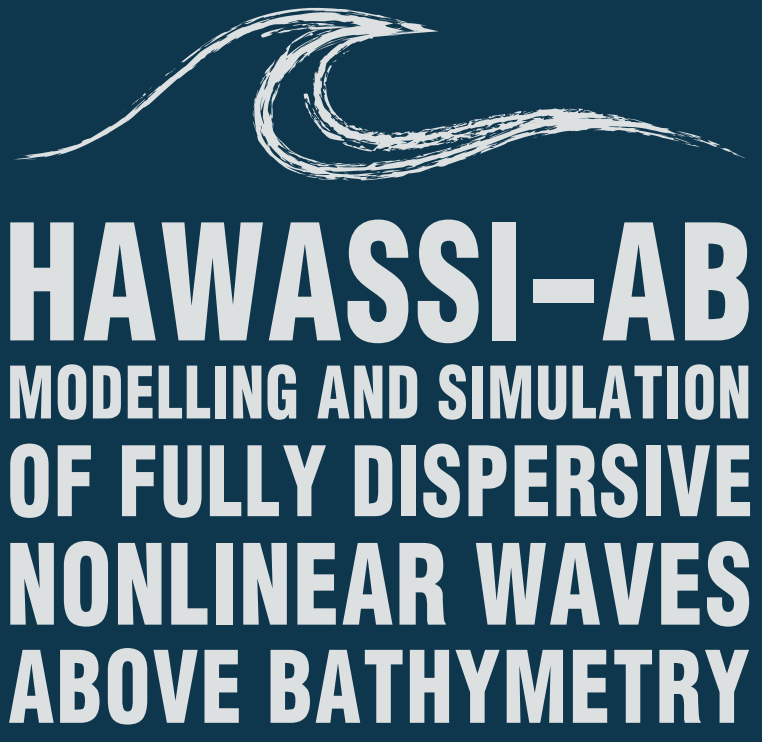

RUDDY KURNIA 


\section{HAWASSI-AB MODELLING AND SIMULATION OF FULLY DISPERSIVE NONLINEAR WAVES ABOVE BATHYMETRY}

Ruddy Kurnia 


\section{Samenstelling promotiecommissie:}

Voorzitter en secretaris:

prof. dr. P. M. G. Apers University of Twente

\section{Promotor}

prof. dr. ir. E. W. C. van Groesen University of Twente

\section{Leden}

prof. dr. S. A. van Gils

University of Twente

prof. dr. A. E. P. Veldman

University of Twente

prof. dr. ir. R. H. M. Huijsmans

Delft University of Technology

prof. dr. F. Dias

University College Dublin, Ireland

prof. dr. B. Jayawardhana

University of Groningen

dr. ir. T. Bunnik

MARIN

\section{UNIVERSITY OF TWENTE.}

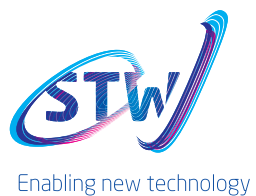

The research presented in this dissertation was carried out at the Applied Analysis group, Departement of Applied Mathematics, Faculty of Electrical Engineering, Mathematics and Computer Science (EEMCS) of the University of Twente, PO Box 217, 7500 AE Enschede, The Netherlands.

This research is supported by the Dutch Technology Foundation STW, which is part of the Netherlands Organisation for Scientific Research (NWO) and partly funded by the Ministry of Economic Affairs (project number 11642).

Copyright (c) 2016, Ruddy Kurnia, Enschede, The Netherlands

Cover: Erika Tivarini, www.erikativarini.carbonmade.com

Printed by Gildeprint, Enschede

ISBN 978-90-365-4039-1

DOI $10.3990 / 1.9789036540391$

http://dx.doi.org/10.3990/1.9789036540391 


\title{
HAWASSI-AB MODELLING AND SIMULATION OF FULLY DISPERSIVE NONLINEAR WAVES ABOVE BATHYMETRY
}

\author{
DISSERTATION
}

to obtain

the degree of doctor at the University of Twente, on the authority of the rector magnificus, prof. dr. H. Brinksma,

\begin{abstract}
on account of the decision of the graduation committee,
\end{abstract} to be publicly defended on Friday 19 February 2016 at 16:45

$$
\text { by }
$$

\section{Ruddy Kurnia}

born on the $1^{\text {th }}$ of May 1987

in Bandung, Indonesia 
Dit proefschrift is goedgekeurd door de promotor prof. dr. ir. E. W. C. van Groesen 
To my parents 



\section{Summary}

Water waves propagating from the deep ocean to the coast show large changes in the profile, wave speed, wave length, wave height and direction. The fascinating processes of the physical wave phenomena give challenges in the study of water waves. The motion can exhibit qualitative differences at different scales such as deep water versus shallow water, long waves versus short waves. Therefore, the existing mathematical models are restricted to the limiting cases. This dissertation concerns the development of an accurate and efficient model that can simulate wave propagation in any range of wave lengths, in any water depth and moreover can deal with various inhomogeneous problems such as bathymetry and walls, leading to wave structure interactions.

The derivation of the model is based on a variational principle of water waves. The resulting dynamic equations are of Hamiltonian form for wave elevation and surface potential with non-local operators applied to the canonical surface variables. The Hamiltonian is the total energy, i.e the sum of kinetic energy and potential energy. Since the kinetic energy cannot be expressed explicitly in the basic variables an approximation is required. The corresponding approximated Hamiltonian leads to approximated Hamilton equations.

The approximate Hamilton equations are expressed in pseudo-differential operators applied to the surface variables. The pseudo-differential operator has a physical interpretation related to the phase velocity. The phase velocity as function of wave length is specified by a dispersion relation. Dispersion is one of the most important physical properties in the description of water waves. Accurate modelling of dispersion is essential to obtain high-quality wave propagation results.

Using spatial-spectral methods and a straightforward numerical implementation, accurate and fast performance of the model can be obtained. Moreover, the spatialspectral implementation with the global pseudo-differential operators or a generalization with global Fourier integral operators (FIO) can retain the exact dispersion property of the model. Other numerical implementations with local differential operators such as finite difference or finite element methods require that the dispersion is approximated by an algebraic function. Such an approximation leads to restrictions on the range of wave lengths that are modelled correctly.

To deal with practical applications, several extensions of the model are imple- 
mented. The model with localization methods in the global FIO can deal with localized effects such as breaking waves, partially or fully reflective walls, submerged bars, run-up on shores, etc. The inclusion of a fixed-structure in the spatial-spectral setting is a challenging task. The method as presented here perhaps serves as a first contribution in this topic.

An extended eddy viscosity breaking model and a breaking kinematic criterion are used for the wave breaking mechanism. The extended eddy viscosity breaking model can deal with fully dispersive waves. The kinematic breaking criterion prescribes that a wave will break when the horizontal particle speed exceeds (a fraction of) the crest speed. A universal or deterministic value of this parameter is not known yet.

In many applications, such as the calculation of wave forces on structures, requires information of interior flow properties. A method to calculate the interior flows in a post-processing step of the Boussinesq model is described.

Performance of the model is shown by comparing the simulation result with measurement data of various long crested cases of breaking and non-breaking waves. The model has been extensively tested against at least 50 measurement data. Moreover, 30 measurement data of wave breaking experiments were designed by the accurate wave model. It will be shown that an efficient and accurate code can optimize the experiments.

The models and methods presented in this dissertation have been packaged as software under the name HAWASSI-AB; here HAWASSI stands for Hamiltonian Wave-Ship-Structure Interaction, while AB stands for Analytic Boussinesq. More information of the software can be found on http://hawassi.labmath-indonesia.org. 


\section{Samenvatting}

Watergolven vertonen gedurende hun reis van de diepe oceaan naar de ondiepe kust grote veranderingen in vorm, snelheid, golflengte en richting. De fascinerende processen van deze fysische golfverschijnselen leiden tot uitdagend onderzoek. De beweging kan leiden tot kwalitatieve verschillen op verschillende schalen van waterdiepte en golflengte. Daarom zijn veel wiskundige modellen beperkt tot limiet gevallen. Dit proefschrift behandelt het ontwikkelen van een nauwkeurig en efficint model dat de voortplanting kan beschrijven en berekenen van golven met willekeurige golflengte boven willekeurige waterdiepte, zelfs in interactie met inhomogeniteiten zoals veranderende bodemdiepte of de aanwezigheid van wanden.

De afleiding van het model is gebaseerd op een variatieprincipe voor watergolven. De resulterende dynamische vergelijkingen zijn een Hamiltons systeem voor de golfhoogte en de oppervlakte potentiaal met niet-lokale operatoren die werken op deze canonieke oppervlakte variabelen. De Hamiltoniaan is de totale energie, de som van kinetische en potentiele energie. Omdat de kinetische energie niet expliciet uitgedrukt kan worden in de basisgrootheden is een benadering vereist. De daarmee corresponderende Hamiltoniaan leidt tot de benaderde Hamilton vergelijkingen.

Deze vergelijkingen zijn uitdrukkingen met pseudo-differentiaal operatoren toegepast op de oppervlakte variabelen. Deze operator is fysisch gerelateerd aan de fase-snelheid. Deze snelheid wordt bepaald door de golflengte via de zogenaamde dispersie-relatie. Dispersie is een van de meest belangrijke eigenschappen in de beschrijving van watergolven, en een goede benadering is essentieel om goede resultaten te verkrijgen voor golfvoortplanting.

Ruimtelijk-spectrale methoden en een directe numerieke implementatie leiden tot nauwkeurige en snelle resultaten. Bovendien kan door de ruimtelijk-spectrale implementatie van de globale pseudo-differentiaal operatoren, of de generalisatie naar Fourier integraal operatoren (FIO), de exacte dispersie-eigenschappen van het model bewaard worden. Overige numerieke implementaties met lokale differentiaal operatoren, zoals eindige-differentie of eindige-element methoden, vereisen een benadering van de dispersie met een algebrasche functie, hetgeen tot beperkingen leidt van de golflengten die nauwkeurig voortgeplant worden.

Om praktische problemen aan te kunnen pakken zijn meerdere uitbreidingen geimplementeerd. Localisatie-methoden voor de globale FIOs maken het mogelijk 
gelocaliseerde effecten te simuleren, zoals brekende golven, gedeeltelijk of volledig reflecterende wanden, onderwaterdrempel, oploop op de kust, etc. Dit toevoegen van vaste structuren in ruimtelijk-spectrale modellen is een uitdagende taak; de bijdragen daaraan die hier worden gepresenteerd zijn misschien de eersten van dit soort.

Een eddy-viscositeits breking model met een kinematisch breking criterium worden gebruikt voor golfbreking; het brekingmodel is uitgebreid zodat het bruikbaar is voor volledig dispersieve golven. Het kinematisch criterium zorgt ervoor dat een golf breekt als de horizontale deeltjessnelheid groter is dan een fractie van de snelheid van de golftop. Een universele of deterministische waarde voor die fractie is nog niet bekend.

In veel toepassingen zijn de interne stromingssnelheden van belang, bijvoorbeeld voor de berekening van krachten op structuren. Er wordt een methode gepresenteerd om de interne stroming te berekenen nadat de oppervlaktegrootheden van het Boussinesq model zijn berekend.

De prestaties van het model zijn aangetoond voor meer dan 50 gevallen door berekende resultaten te vergelijken met meetdata van experimenten van langkammige golven met of zonder breking. Bovendien zijn met de software 30 experimenten van golfbreking ontworpen, waarmee aangetoond wordt dat daarmee het experimenteren geoptimaliseerd kan worden.

Het model inclusief alle nieuwe methoden is als software beschikbaar onder de naam HAWASSI-AB. De afkorting HAWASSI staat voor Hamiltonian Wave-ShipStructure Interaction, en AB voor Analytic Boussinesq; meer informatie is te verkrijgen op http://hawassi.labmath-indonesia.org. 


\section{Contents}

Summary vii

Samenvatting ix

$\begin{array}{lll}1 & \text { Introduction } & 1\end{array}$

1.1 A historical note on the study of water waves . . . . . . . . . . 3

1.2 Variational water wave modelling . . . . . . . . . . . . . . 6

1.3 Contributions in this dissertation $\ldots \ldots \ldots \ldots \ldots$

1.4 Outline of the dissertation . . . . . . . . . . . . . . . 11

2 High order Hamiltonian water wave models 13

2.1 Introduction . . . . . . . . . . . . . . . . . . . . . . . . 14

2.2 Variational wave description $\ldots \ldots \ldots \ldots \ldots \ldots \ldots$

2.2 .1 Hamiltonian formulation . . . . . . . . . . . . . . . 16

2.2 .2 Consistent approximations . . . . . . . . . . . . . . . . 17

2.2 .3 Hybrid Spatial Spectral implementation . . . . . . . . . . . 20

2.3 Wave-breaking mode] . . . . . . . . . . . . . . . . . . . . . 21

2.3 .1 Eddv-viscosity model . . . . . . . . . . . . . . . . . . 21

2.3 .2 Kinematic breaking criterion . . . . . . . . . . . . . . 23

2.3 .3 Alternative viscosity model . . . . . . . . . . . . . 25

2.4 Numerical implementation . . . . . . . . . . . . . . . . . . . . 26

2.4 .1 Damping zones . . . . . . . . . . . . . . . . . . 27

2.4 .2 Nonlinear wave generation . . . . . . . . . . . . . . 27

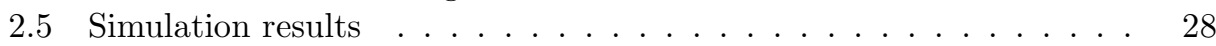

2.5 .1 Irregular wave breaking over a flat bottom . . . . . . . . 28

2.5 .2 Wave breaking over a bar . . . . . . . . . . . . . 36

2.6 Conclusion and remarks . . . . . . . . . . . . . . . 41

3 Localization for spatial-spectral implementations 43

3.1 Introduction . . . . . . . . . . . . . . . . 44

3.2 Spatial-spectral modelling within the Hamiltonian structure . . . . . 45 


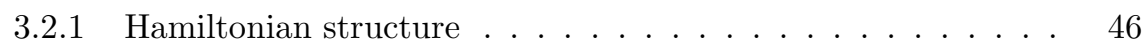

3.2.2 Limiting cases . . . . . . . . . . . . . . . . . . . 47

3.2.3 Second order accurate approximation above bathymetry . . . 48

3.2.4 Wave breaking and bottom friction . . . . . . . . . . . . . . 49

3.2.5 Internal flow and pressure . . . . . . . . . . . . . . . . . . . 50

3.3 Localization . . . . . . . . . . . . . . . . . . . . 51

3.3.1 Partially reflecting wall ... . . . . . . . . . . . 51

3.3.2 Frequency dependent reflecting wall . . . . . . . . . . . . 52

3.3.3 Run-up on coast . . . . . . . . . . . . . . . . . . . . . . . . . . . . . . . . . 52

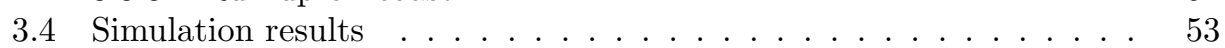

$3.4 .1 \quad$ Irregular waves running up a slope . . . . . . . . . . . . . . . . 53

3.4.2 Irregular wave breaking over a bar . . . . . . . . . . . . . . . . . 55

3.4.3 Harmonic breaking wave running up a coast . . . . . . . . . . 57

3.4.4 Wave-wall interactions . . . . . . . . . . . . . . . 59

3.4.5 Dam-break problem . . . . . . . . . . . . . . 63

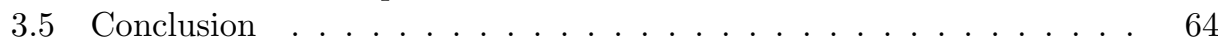

4 Design of wave breaking experiments and a-posteriori simulations 65

4.1 Introduction . . . . . . . . . . . . . . . . 65

4.2 Experimental set up . . . . . . . . . . . . . . . . . . 66 66

4.3 Simulation model . . . . . . . . . . . . . . . . . . . . . . 67

4.4 Design and reconstruction . . . . . . . . . . . . . . . . . . . . . . . . . 68

4.4 .1 Design cases ... . . . . . . . . . . . . . . . . . 68 68

4.4.2 Reconstruction cases . . . . . . . . . . . . . . . . . . 70

4.5 Conclusions ............................ 71

5 Conclusions and recommendations $\quad 75$

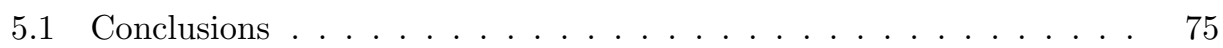

5.2 Recommendations . . . . . . . . . . . . . 76

\begin{tabular}{|ll|}
\hline Appendix A Supplementary files of the experiments & $\mathbf{7 7}$
\end{tabular}

A.1 The characteristic quantities of the designed waves . . . . . . . . . . 77

A.2 Comparison of experiments and a-priori simulations . . . . . . . . . 79

A.3 Comparison of experiments and a-posteriori simulations . . . . . . . 82

\begin{tabular}{lr}
\hline Bibliography & 113
\end{tabular}

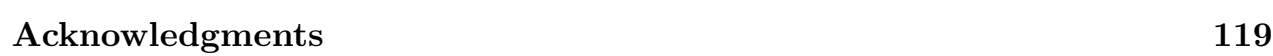

\begin{tabular}{|lr}
\hline About the author & 121
\end{tabular} 


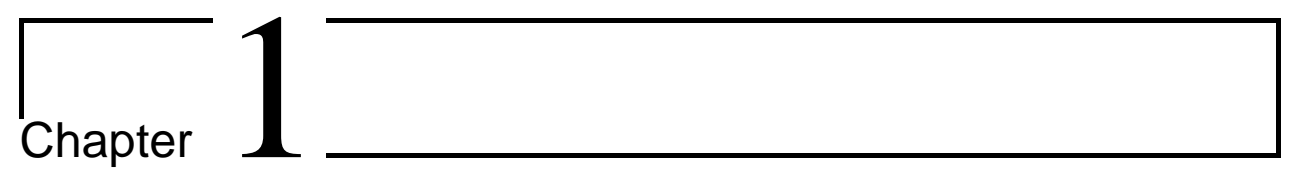

\section{Introduction}

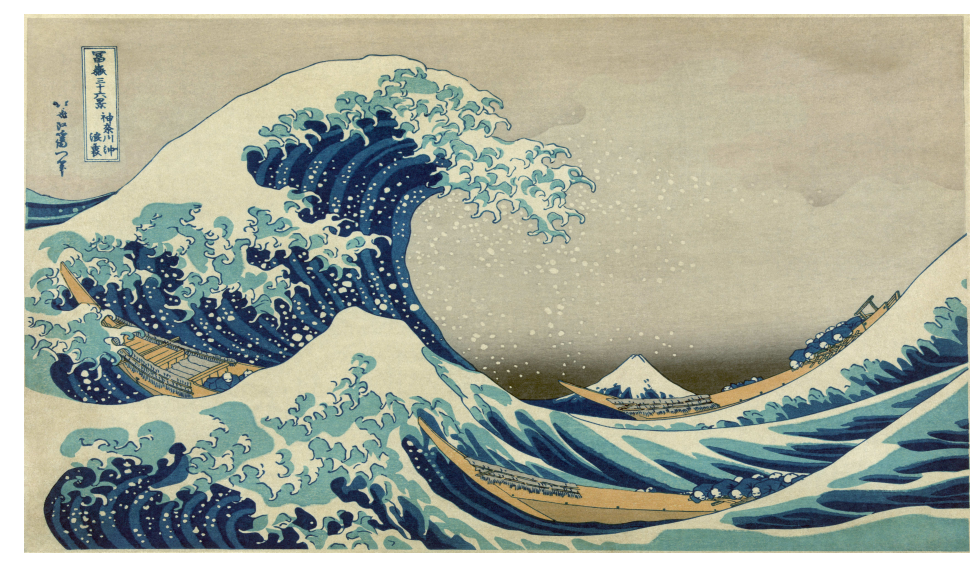

Figure 1.1: The Great Wave off Kanagawa, by Katushika Hokusai (18 ${ }^{\text {th }}$ century) (source: www.wikipedia.org)

Ocean waves are fascinating. The wind blowing over the sea surface generates wind waves. During storms, waves can become very high and develop foamy crests with very complex patterns. Waves approaching the shore get higher and steeper and may break to form waves that are spectacularly used by surfers. The breaking of large oceanic waves has drawn the most attention of human beings to observe this magnificent phenomenon. These natural processes have repeatedly been the themes in paintings. The Great Wave off Kanagawa (Fig. 1.1) is a well-known paintings, published in $18^{\text {th }}$ century by Katsushika Hokusai. The wave has been discussed in scientific notes of Cartwright and Nakamura, 2009, Dudley et al., 2013]. It is stated that the location of the wave is estimated to be $3 \mathrm{~km}$ offshore Tokyo Bay. The estimated wave height of around $10 \mathrm{~m}$ leads to the conclusion that this would be a wave of exceptionally large amplitude for this area and would likely be a rogue 
or freak wave. No less important are the scientific studies or concept of the wave phenomena and the ocean as well.

For thousands of years, people have been depending on the ocean as a source of food and mineral, and as relatively easy medium for transport of people and goods. Nowadays, with developing of knowledge and technology, the ocean gives even more benefits. Resources of renewable energy such as wind farms, tidal and wave energy are mainly located in coastal areas. Moreover, coastal areas are centres of industrial activities, products and therefore money flows into countries through ports. This leads to the fact that half of the world population lives less than $150 \mathrm{~km}$ from the coast.
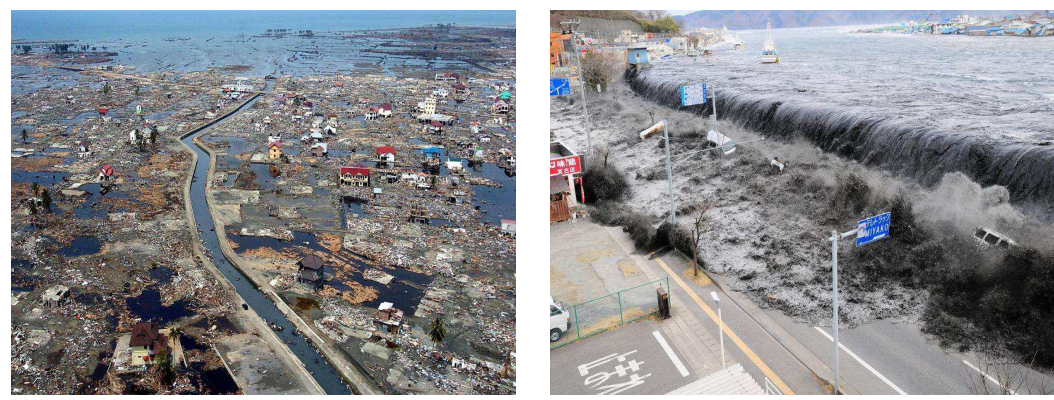

Figure 1.2: At the left: a photo taken on January 5, 2005 of the devastated district of Banda Aceh, Indonesia in the aftermath of the December 26, 2004 tsunami. Credit: Choo Youn-Kong/AFP/Getty Images. Source: theatlantic.com. At the right: Tsunami wave approaches Miyako city in Japan on 11 March 2011. Credit: Mainichi Shimbun /Reuters. Source: reuters.com.
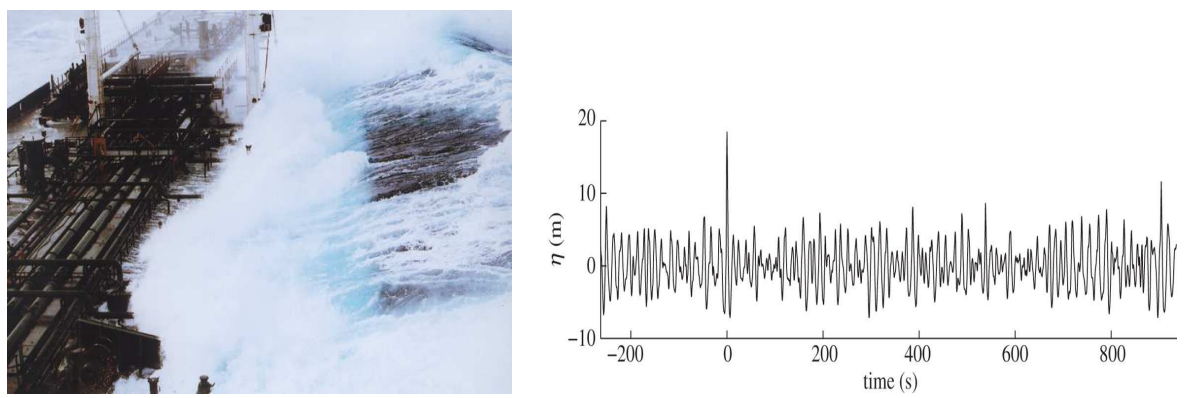

Figure 1.3: At the left: A rogue wave reaching a height of $18 \mathrm{~m}$ hit a tanker headed south from Valdez, Alaska in February 1993. Credit: Captain Roger Wilson, NOAA National Weather Service Collection. At the right: The surface elevation time history recorded at the Draupner platform, which includes the New Year Wave. Source: [Adcock et al., 2011].

Apart from the profits, waves can also give problems. High waves during storms or caused by bathymetry or by collision against constructions or by undersea earthquakes can do great harm to ships, constructions and to people living near the coast. 
On 26 December 2004, a $\mathrm{M}_{w} 9.1$ undersea megathrust earthquake at the west coast of northern Sumatra, Indonesia generated a series of devastating tsunamis along the coasts of the Indian ocean. The series of waves reached the coasts of Banda Aceh (northwest corner of Sumatra) within 15 min after the earthquake, thus inundating $100 \mathrm{~km}^{2}$ of land. The waves were $5-30 \mathrm{~m}$ high at the coast and runup to $51 \mathrm{~m}$ and $6 \mathrm{~km}$ inland Paris et al., 2007. The reported number of casualties were approximately 230.000 killed in Indonesia, at least 29,000 killed in Sri Lanka, more than 10,000 in India, more than 5,000 in Thailand, and 82 killed in Maldives, and more than 22,000 are still missing Kawata et al., 2005].

On 11 March 2011, a $\mathrm{M}_{w} 9.0$ earthquake in the Pacific ocean close to Tohoku generated tsunami waves. The waves inundated the area with wave heights up to $15 \mathrm{~m}$, runup height reached over $39 \mathrm{~m}$ and $6 \mathrm{~km}$ inland. Over 14.000 people were reported as dead and over 11.000 were missing [Mimura et al., 2011].

Furthermore, extreme waves, also known as rogue waves or freak waves, have been major causes of numerous accidents of oil-platforms and ships. Practically, a rogue wave is expected to be at least twice larger than the significant wave height.

In February 1993, a rogue wave in the Gulf of Alaska was photographed by Captain Roger Wilson Wilson, 1993]. The wave was reaching a height of $18 \mathrm{~m}$ above $7.6 \mathrm{~m}$ water depth. The rogue wave hit a tank ship on the starboard side when the ship was heading to south from Valdez, Alaska.

On 1 January 1995, the "New Year wave" was recorded in the North Sea at the Statoil-operated "Draupner" platform Adcock et al., 2011]. The Draupner wave was the first rogue wave to be detected by a measuring instrument. It was recorded that the crest height was $18.6 \mathrm{~m}$, wave height $25.6 \mathrm{~m}$ above $70 \mathrm{~m}$ water depth. Fortunately this wave did not cause substantial damage, but attracted attention of the scientists to this problem. Since then, numerous accidents of oil-platforms and ships have been linked to the rogue wave occurrence.

Nikolkina and Didenkulova 2011] collected evidence of rogue wave existence during the 5 year period, 2006-2010. From the total of 131 reported events, 78 were identified as evidence of rogue waves. Only events associated with damage and human loss were included. It is also stated that the extreme waves cause more damage in shallow waters and at the coast than in the deep sea.

Therefore, a sustainable and safe development of the oceanic and coastal areas is of paramount importance. The fascination and the practical relevance have been motivating extensive study of water waves, probably as long as people live on earth but certainly in the past centuries. The past and recent studies of water waves are summarized in the following sections. Section 1.1 summarizes the extensive study in the past centuries, and Section 1.2 gives a description of variational water wave modelling. Highlights of the contributions of this dissertation are presented in Section 1.3. The outline of the dissertation will finish this chapter.

\subsection{A historical note on the study of water waves}

The water wave problem in fluid mechanics has been known since more than three hundreds years Craik, 2004]. In 1687, Isaac Newton attempted a theory of water 
waves with an analogy of a fluid oscillations in a U-shaped tube. He correctly deduced that the frequency of deep-water waves must be proportional to the inverse of the square root of the breadth of the wave.

In 1757, Leonhard Euler published a physically and mathematically successful description of the behaviour of an idealized fluid (inviscid flow). These Euler equations represent conservation of mass (continuity) and momentum. The Euler equations became the foundation of a realistic description of water which was derived 65 years later by Claude Navier. That is now known as the Navier-Stokes equations.

Pierre-Simon Laplace (1776) derived a fundamental equation of tidal motion. He focused on free surface propagation, which only occurs if the cause of the wave is localized in space and time. This leads to the general initial value problem: Given any localized initial disturbance of the liquid surface, what is the subsequent motion? Cauchy and Poisson later addressed this problem.

Later, Joseph Louis Lagrange (1781) also worked on the governing equation of linear water waves and obtained the solution in the limiting case of shallow water. He found that the speed of propagation of waves will be independent of wavelength and proportional to the square root of water depth; that is $(g h)^{1 / 2}$ where $g$ is the gravitational acceleration and $h$ the water depth.

In December 1813, the French Académie des Sciences announced a mathematical prize competition on wave propagation on infinite depth. In 1816 Cauchy won the prize and his work was published in 1827. Independently, Poisson, who was one of the judges, deposited a memoir of his own work that was published in 1818 . The Cauchy-Poisson analysis is now acknowledged as an important milestone in the mathematical theory of initial-value problem.

Cauchy employed Fourier transform in analysing the Laplace equation for the velocity potential $\Phi(x, y, z)$ with $x, y$ the horizontal coordinates and $z$ the vertical coordinate

$$
\frac{\partial^{2} \Phi}{\partial x^{2}}+\frac{\partial^{2} \Phi}{\partial y^{2}}+\frac{\partial^{2} \Phi}{\partial z^{2}}=0
$$

incorporating the linearized free surface condition, with $g$ the acceleration of gravity,

$$
\frac{\partial^{2} \Phi}{\partial t^{2}}+g \frac{\partial \Phi}{\partial z}=0
$$

Cauchy then takes the second time-derivative of Eq. 1.2 as

$$
\frac{\partial^{4} \Phi}{\partial t^{4}}=-g \frac{\partial^{3} \Phi}{\partial t^{2} \partial z}=-g \frac{\partial^{3} \Phi}{\partial z \partial t^{2}}=g^{2} \frac{\partial^{2} \Phi}{\partial z^{2}} .
$$

Using Eq. 1.1 Cauchy's equation is given by

$$
\frac{\partial^{4} \Phi}{\partial t^{4}}+g\left(\frac{\partial^{2} \Phi}{\partial x^{2}}+\frac{\partial^{2} \Phi}{\partial y^{2}}\right)=0 .
$$

For a periodic wave of form $\exp \left[i\left(k_{x} x+k_{y} y-\omega t\right)\right]$, the correct dispersion relation of deep-water waves can be obtained as

$$
\omega^{2}=g\left(k_{x}^{2}+k_{y}^{2}\right)^{1 / 2} .
$$


However, Cauchy's equation is only valid in the case of infinite depth since the bottom condition is neglected.

In 1834, solitons, waves that propagate with constant speed and constant shape, were observed for the first time by the British scientist John Scott Russell. He was watching a barge being towed along a canal between Glasgow and Edinburg. On its sudden stop, a wave was observed, that propagated for nearly a mile with only little change of form. This phenomenon inspired him to perform experiments. Substantial reports by Russell and Robinson were published in 1837 and 1840 . Russell wrote a brief supplementary report (1842) and then his major "Report on Waves" (1844). Later, Boussinesq in 1872, and Korteweg and de Vries in 1895 produced theoretical results of the soliton wave.

In 1841, George Biddel Airy published an influential article 'Tides and Waves'. His work became a major contribution of water wave theory. He gave a complete formulation of linear propagation of gravity waves. In his formulation, an impermeable boundary-condition was taken into account. The formulation is given as follows

$$
\begin{aligned}
\frac{\partial^{2} \Phi}{\partial x^{2}}+\frac{\partial^{2} \Phi}{\partial y^{2}}+\frac{\partial^{2} \Phi}{\partial z^{2}}=0 & \text { for }-h \leq z \leq 0 \\
\frac{\partial \Phi}{\partial t}+g \eta=0 & \text { for } z=0 \\
\frac{\partial \eta}{\partial t}=\frac{\partial \Phi}{\partial z} & \text { for } z=0 \\
\frac{\partial \Phi}{\partial z}=0 & \text { for } z=-h
\end{aligned}
$$

in which $\Phi$ is the fluid potential, $\eta$ the surface elevation, $h$ the water depth and $g$ the gravity acceleration.

Airy's equations represent incompressible and irrotational flow in the interior (Eq. 1.5), a dynamic free surface condition (Eq. 1.6), a kinematic free surface condition (Eq. 1.7) and an impermeable bottom condition (Eq. 1.8). Observe that the free surface conditions are actually the Cauchy condition (Eq. 1.2). Airy's linear theory produces a correct dispersion relation for a propagating monochromatic wave, $\eta(x, y, t)=a \cos \left(k_{x} x+k_{y} y-\omega t\right)$ with $a$ the amplitude, $k=\sqrt{k_{x}^{2}+k_{y}^{2}}$ the wave number and $\omega$ the angular frequency. The dispersion relation of wave propagation above a depth $h$ is given by

$$
\omega^{2}=g k \tanh (k h) .
$$

The dispersion relation also tells that the wave speed (which is the quotient of $\omega$ and $k$ ) depends on wavelength. As a consequence, the shorter waves travels slower.

The work of Airy on the linear wave theory and the remarkable experiments of Russel motivated Stokes to investigate the water wave problem. In 1847, Stokes published his work on nonlinear wave theory that is accurate up to third order in wave steepness $(k . a)$. He showed surface elevation $\eta$ in a plane wavetrain on deep water could be expanded in powers of the amplitude $a$ as

$$
\eta(x, t)=a\left[\cos (k x-\omega t)+\frac{1}{2} k a \cos 2(k x-\omega t)+\frac{3}{8}(k a)^{2} \cos 3(k x-\omega t)+\cdots\right]
$$


where $\omega^{2}=g k\left(1+(k a)^{2}+\cdots\right)$ the nonlinear dispersion. As a consequence of the nonlinear dispersion, the steeper the wave the faster it travels. He also showed that a wave with maximum height has a crest angle of $120^{\circ}$. The nonlinear effect influences the wave shape with sharp and higher crest and flatter at the trough.

As a response to the observation and the experiment of soliton wave by John Scott Russel, Boussinesa [1872] derived equations that are now known as the Boussinesq equations. Boussinesq simplified the Euler equations for irrotational, incompressible fluid. He approximated the depth dependence of the Laplace equation in the interior fluid potential. In the approximation, a Taylor expansion up to a certain order around still water level is applied at the velocity potential function with incorporating the frequency dispersion. That leads to bi-directional and dispersive dynamic equation for the surface elevation and the velocity. These 'classical' Boussinesq equations are valid for weakly nonlinear and fairly long waves. Of major importance is the fact that the whole dynamics is expressed solely by quantities at the surface, without any explicit equations for the interior flows. Nowadays, therefore, such models are more generally called Boussinesq (type of) equations.

Korteweg and de Vries [1895] derived a simplified Boussinesq equation. The simplification was obtained in such a way that the bi-directional dynamic equations lead to one unidirectional dynamic equation. The KdV equation is valid for weakly nonlinear and weakly dispersive long waves and can be expressed as

$$
\eta_{t}+\left(c_{0}+c_{1} \eta\right) \eta_{x}+\nu \eta_{x x x}=0
$$

where $c_{0}, c_{1}$ and $\nu$ are constants. The $\mathrm{KdV}$ equation has the same properties as the Boussinesq equation that both have (periodic) cnoidal and soliton profiles as solution. These theoretical results answered the observation and the experimental result of solitons by Scott Russel.

\subsection{Variational water wave modelling}

Many mathematical models of surface gravity wave have been developed recently. Most of the effort is to improve the accuracy of the model in terms of dispersion and nonlinear properties. A fascinating feature of the study of water waves is that the motion can exhibit qualitative differences at different scales such as deep water versus shallow water, long wavelength versus short wavelength. Therefore the existing wave models were constructed by various approximations to the limiting cases.

In shallow water, there are equations of Boussinesd 1872, Korteweg and de Vries 1895], Beniamin et al. [1972], Serre [1953], Green and Naghdi [1976], Camassa and Holm [1993], and others. On finite depth and deep water, there are equations of [Stokes, 1847], nonlinear Schrödinger type of Dysthe [1979], Peregrine [1983] and others. Generally, these equations are valid for a limited range of the the relative water depth ( $k h$, in which $k$ the wave number, $h$ the water depth). Most of the models were derived using some perturbation techniques that are valid for relatively small amplitude.

However, in many applications it is desired to use a wave model that is uniformly valid for all depths and also accurate for large amplitudes. To that end, a different 
approach of modelling, the so-called variational formulation is used in this work. In this section, the development of the variational formulation of water waves is summarized.

Luke [1967] formulated a Lagrangian variational description of the motion of surface gravity waves on an incompressible and irrotational fluid with a free surface as

$$
\operatorname{Crit}_{\Phi, \eta} \int \mathcal{P}(\Phi, \eta) d t \quad \text { where } \quad \mathcal{P}(\Phi, \eta)=\int\left[\int_{-h}^{\eta}\left(\partial_{t} \Phi+\frac{1}{2}\left|\nabla_{3} \Phi\right|^{2}+g z\right) d z\right] d \mathbf{x}
$$

The variables in this variational principles are the surface elevation $\eta$ (depending on the two horizontal dimensions $x$ and $y$ ) and the fluid potential $\Phi$ inside the fluid, so depending on horizontal and vertical dimensions. Note that this is a 'pressure principle' since the integrand $\mathcal{P}$ denotes the pressure in the fluid, according to Bernoulli's formulation of the Euler equation for irrotational fluid. The pressure principle has been remarked before by Bateman [1929] but without considering variations of the free surface $\eta$.

The following is a derivation of the water wave problem from the Luke's Lagrangian functional. The vanishing of the first variation of the functional with respect to variation $\delta \Phi$ in $\Phi$ leads to

$$
\int d t \int d \mathbf{x}\left[\int_{-h}^{\eta} \partial_{t}(\delta \Phi)+\nabla_{3} \Phi \cdot \nabla_{3}(\delta \Phi) d z\right]=0 .
$$

It can be rewritten by applying Leibniz's integral rule for the first term

$$
\int_{-h}^{\eta} \partial_{t}(\delta \Phi)=\partial_{t}\left(\int_{-h}^{\eta}(\delta \Phi) d z\right)-(\delta \Phi)_{z=\eta} \partial_{t} \eta-(\delta \Phi)_{z=-h} \partial_{t} h
$$

and the use Gauss's theorem for partial integration

$$
\int d \mathbf{x}\left[\int_{-h}^{\eta} \nabla_{3} \Phi \cdot \nabla_{3}(\delta \Phi) d z\right]=-\int d \mathbf{x}\left[\left(\nabla_{3} \cdot \nabla_{3} \Phi\right) \delta \Phi d z\right]+\int d \mathbf{x}\left[\left(\partial_{N} \Phi\right) \delta \Phi\right]_{z=-h}^{z=\eta} .
$$

Here a boundary term at the lateral boundaries has been neglected. Then the vanishing for all variations $\delta \Phi$ leads to Laplace equation in the interior fluid, the impermeable bottom and the kinematic free surface conditions. The equations are explicitly expressed as

$$
\begin{aligned}
& \Delta_{3} \Phi=0 \text { for } \quad-h<z<\eta \\
& \nabla_{3} \Phi \cdot N_{b}=0 \text { at } z=-h, \text { where } N_{b}=(-\nabla h,-1) \\
& \nabla_{3} \Phi \cdot N_{s}=\partial_{t} \eta \text { at } z=\eta \text {, where } N_{s}=(-\nabla \eta, 1) \text {. }
\end{aligned}
$$

The dynamic free surface condition can be obtained by vanishing the first variation of the pressure principle with respect to variations $\delta \eta$ in $\eta$, leading to the result as

$$
\partial_{t} \Phi+\frac{1}{2}\left|\nabla_{3} \Phi\right|^{2}+g \eta(\mathbf{x}, t)=0 \text { at } z=\eta(\mathbf{x}, t)
$$


That is Bernoulli's equation which states that the pressure at the surface of the water should vanish, as the assumed pressure condition.

Zakharov [1968] and later independently Broer, 1974] showed a Hamiltonian structure for the dynamic of surface gravity waves. The relation between the Hamiltonian structure of the water wave dynamics and Luke's Lagrangian variational principle was shown by Miles 1977.

Miles reformulated Luke's variational principle in the basic (canonical) variables: the surface elevation $\eta(\mathbf{x}, t)$ and the surface potential $\phi(\mathbf{x}, t)=\Phi(\mathbf{x}, z=\eta(\mathbf{x}, t), t)$. It is known that by prescribing the surface potential, the solution of the interior Laplace problem is uniquely determined and moreover the dimension reduction is achieved. Then, using Leibniz's integral rule

$$
\int_{-h}^{\eta} \partial_{t} \Phi d z=\partial_{t}\left(\int_{-h}^{\eta} \Phi d z\right)-\phi \partial_{t} \eta-\left.\Phi\right|_{z=-h} \partial_{t} h
$$

the functional $\mathcal{P}(\Phi, \eta)$ can be rewritten as

$$
\begin{aligned}
\mathcal{P}(\Phi, \eta) & =-\left[\int \phi \partial_{t} \eta d \mathbf{x}-\frac{1}{2} \int d \mathbf{x} \int\left|\nabla_{3} \Phi\right|^{2} d z-\frac{1}{2} \int g\left(\eta^{2}-h^{2}\right) d \mathbf{x}\right] \\
& +\partial_{t} \int d \mathbf{x} \int_{-h}^{\eta} \Phi d z .
\end{aligned}
$$

By neglecting an unimportant term at the boundary of the time interval and the constant term $\int h^{2} d \mathbf{x}$, this then leads to the canonical action principle for a Hamiltonian $\mathcal{H}(\phi, \eta)$

$$
\operatorname{Crit}_{\phi, \eta} \int d t\left[\int \phi \partial_{t} \eta d \mathbf{x}-\mathcal{H}(\phi, \eta)\right] \text {. }
$$

The Hamiltonian is, just as in Classical Mechanics, the total energy, i.e. the sum of the kinetic energy and the potential energy

$$
\mathcal{H}(\phi, \eta)=\mathcal{K}(\phi, \eta)+\frac{1}{2} \int g \eta^{2} d \mathbf{x}
$$

The kinetic energy is given by

$$
\mathcal{K}(\phi, \eta)=\min _{\Phi}\left\{\frac{1}{2} \iint_{-h}^{\eta}|\nabla \Phi|^{2} d z d \mathbf{x} \mid \Phi(\mathbf{x}, z=\eta)=\phi\right\}
$$

Observe that the $\Phi$ satisfies the Laplace equation in the interior with vanishing normal derivative at the impermeable bottom and at lateral boundaries.

The governing equations, the so-called Hamilton equations, are obtained by variation with respect to $\phi$ and $\eta$ in the action principle and are explicitly given by

$$
\begin{aligned}
& \partial_{t} \eta=\delta_{\phi} \mathcal{H}(\phi, \eta) \\
& \partial_{t} \phi=-\delta_{\eta} \mathcal{H}(\phi, \eta) .
\end{aligned}
$$


The reformulation of Luke's principle above results a formulation in Hamiltonian form with the basic variables that depend only on horizontal dimensions $\mathbf{x}$ and time $t$ but not on the vertical dimension $z$ any more. However, the Hamiltonian contains the kinetic energy functional $\mathcal{K}(\phi, \eta)$ (Eq. 1.16) which cannot be expressed explicitly in the basic variables, leading to the essential problem of surface wave theory. Therefore an approximation is needed.

The idea of the approximation is to restrict the minimization of the kinetic energy (Eq. 1.16) to a class of wave motion (for instance small amplitude and/or long waves, etc.) that one is interested in. As a consequence, the corresponding approximate Hamiltonian leads to the approximate Hamilton equations. Note that approximating within the variational structure has the advantage that all special properties that are a consequence of the variational form are retained. But the quality depends on the approximate kinetic energy functional for the class of waves under investigation.

Recently, the variational formulation has led to two classes of surface wave model that are known as Variational Boussinesq Model (VBM) and Analytic Boussinesq (AB) model. As introduced by Klopman et al. [2010], a Ritz method is applied in the vertical structure of the fluid potential $\Phi$ in the kinetic energy functional and then the approximate Hamilton equations leads to the VBM model. An optimization of the model has been obtained by Lakhturov et al. 2012] to deal with broad-band waves. The optimization is applied such that the error between the exact and the VBM kinetic energy is minimum. The VBM with finite element numerical implementation performs reasonably well in several practical applications as shown in Adytia and van Groesen, 2012, Adytia, 2012.

The Analytic Boussinesq (AB) model was derived by van Groesen and Andonowati [2007]. In the derivation, the kinetic energy is approximated around the still water level, keeping the exact dispersion properties. Applying uni-directionalization in the Action principles leads to the $\mathrm{AB}$ equation as the (improved) KdV type of equation. The $\mathrm{AB}$ equation is accurate up to and including second-order in wave-height and applicable for finite and infinite-depth dispersion. A spectral implementation makes it possible to treat the non-algebraic dispersion relation in an exact way above flat bottom. A quasi-homogeneous approximation can be applied to deal with varying bottom as shown in van Groesen and Andonowati, 2011, van Groesen and van der Kroon, 2012].

The AB model shows good accuracy and fast calculations in the most practical cases as presented in van Groesen et al. [2010] for the case of narrow spectra of bichromatic waves and Latifah and van Groesen 2012] for the case of very broad spectra of focussing wave group and the New Year wave. An extension of the AB model from one to two horizontal dimensions has been done as presented in Lie and van Groesen [2010], Lie [2013]. The 2D AB model leads to the (improved) Kadomtsev-Petviashvili (KP) type of equation.

\subsection{Contributions in this dissertation}

Practical applications in oceanic and coastal waves motivates the writer to contribute in developing a tool that accurately and efficiently calculates, simulates and analy- 
ses wave propagation. That means for efficiency and safety in design of harbours, breakwater, ships, off-shores structures, etc..

There are two main contributions in this dissertation. First, accurate dispersive wave models with high order nonlinearity including wave breaking mechanism that are applicable for any water depth. Second, localization methods in the spatial-spectral implementation to deal with various inhomogeneous problems such as bathymetry and walls, leading to wave structure interactions.

The accurate dispersive wave models, the so called ABHS (Analytic Boussinesq Hamiltonian system) Kurnia and van Groesen, 2014a are an extension of the unidirectional AB wave model van Groesen and Andonowati, 2007] to bi-directional waves with high order nonlinearity. As in the $\mathrm{AB}$ model an expansion around the still water level is used to approximate the exact kinetic energy to any desired order in the wave elevation, keeping the exact dispersion properties. The approximation leads to dynamic equations that are expressed in a phase velocity operator that depends on the varying bottom in second, third and fourth order surface elevation.

A spatial-spectral implementation using pseudo-differential operator (PDO) or the generalization with Fourier integral operator (FIO) avoids an approximation of the dispersion relation. Other numerical implementations with local differential operators i.e. finite differences, finite element, etc. require that the dispersion is approximated by an algebraic function. The approximation of the dispersion relation leads to restrictions on the range of wave lengths that are modelled correctly.

An extension of the eddy-viscosity breaking model [Kennedy et al., 2000] is implemented. The extension makes it possible to deal with fully dispersive waves. As trigger mechanism, a kinematic breaking criterion is used. The kinematic breaking criterion requires that the wave will break when the horizontal particle speed exceeds (a fraction of) the crest speed.

\section{2 .}

Details of derivation and validations of the ABHS models can be found in Chapter

Generalizing the PDO associated with the linear phase speed operator of Airy's theory by the FIO associated with the nonlinear phase speed operator leads to a generalized AB model. The generalized AB model with localization methods in the global FIO performs well for even the most difficult cases such as waves interaction with coasts, submerged bars, fully or partially reflective walls. The inclusion of such fixed structures in a spatial-spectral setting perhaps serves as first contribution in this topic.

In Boussinesq-type of models, the output of simulations are the quantities of the surface variables, wave elevation and surface potential. However, in many applications it is desired to have information in the interior. A method to calculate or recover the internal flow properties is described.

Details of derivation, validations and applicability of the generalized AB model can be found in Chapter 3 .

Performance of the models is shown by comparing the simulation result with measurement data of long crested cases of breaking and non-breaking waves. The models have been validated with at least 50 measurement data and presented in several publications Kurnia and van Groesen, 2014a, b, 2015a, b. c, Kurnia et al., 2015. 30 measurement data of wave breaking experiments were designed using the ABHS 
models. The experiments were conducted in a wave-tank of Technical University of Delft (TUD). The design of wave breaking experiments and a-posteriori simulations are presented in Chapter 4 .

The models have been packaged as software under the name HAWASSI-AB, here HAWASSI stands for Hamiltonian Wave-Ship-Structure Interaction, while AB stands for Analytic Boussinesq. Presently the software is for simulation of wavestructure interactions; coupled wave-ship interaction is foreseen in future releases. The copyright of HAWASSI software is with LabMath-Indonesia, an independent research institute in Bandung, Indonesia. Further information of the software can be found on http://hawassi.labmath-indonesia.org, where also a demo version can be downloaded.

\subsection{Outline of the dissertation}

This dissertation consists of an introduction, three main chapters, conclusions and recommendations, and an appendix. An overview of the contents of each chapter is briefly described below.

The previous sections gave an introduction of this dissertation.

In Chapter 2, high order Analytic Boussinesq (AB) models are derived consistently from variational principle. This leads to Hamilton equations that are expressed in phase speed operator that depends on the varying bottom in second, third and fourth order surface elevation. A spatial-spectral implementation avoids an approximation of the dispersion relation, leading to the phase speed that is exact. An extended eddy viscosity breaking model is implemented as energy dissipation mechanism. A kinematic breaking criterion is used to detect the initiation breaking events. Irregular wave breaking above deep water and a plunging harmonic wave breaking over a bar are test cases to validate the simulations with the measurements data.

In Chapter 3, a generalized AB model is expressed in the Fourier-integral operators (FIO) associated with the nonlinear phase speed operator that is applied to the surface variables. The generalized AB model with localization methods in the global FIO can deal with the localized effects of partially or fully reflective walls, run-up on a coast, submerged bars, and the dam-break problem. A method to recover the internal flow properties from the surface wave models will be described. The model is validated with measurement data or analytical solutions for various test cases.

In Chapter 4, performance of the $\mathrm{AB}$ model in the design and reconstruction of wave breaking in a wave tank is presented. It is shown that the use of an efficient simulation code can optimize the experiments by designing the influx such that waves will break at a predefined position. A total of 30 different experiments were conducted but only three cases are presented in this Chapter. The information of designed waves and full comparison of experiments and simulations can be found in Appendix A.

Finally, conclusions and recommendations for future work will be discussed in Chapter 5. 



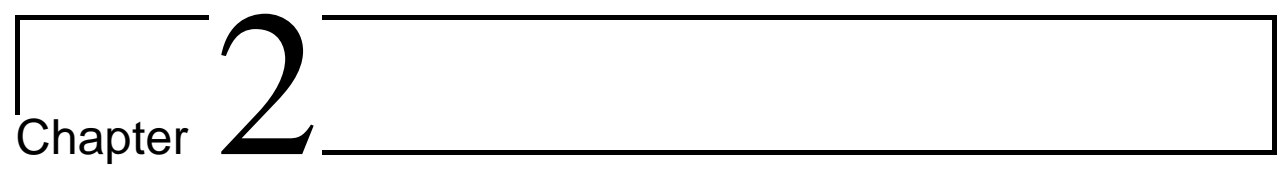

\title{
High order Hamiltonian water wave models with wave-breaking mechanism 1
}

\begin{abstract}
Based on the Hamiltonian formulation of water waves, using Hamiltonian consistent modelling methods, we derive higher order Hamiltonian equations by Taylor expansions of the potential and the vertical velocity around the still water level. The polynomial expansion in wave height is mixed with pseudo-differential operators that preserve the exact dispersion relation. The consistent approximate equations have inherited the Hamiltonian structure and give exact conservation of the approximate energy. In order to deal with breaking waves, we extend the eddy-viscosity model of Kennedy et al. [2000] to be applicable for fully dispersive equations. As breaking trigger mechanism we use a kinematic criterion based on the quotient of horizontal fluid velocity at the crest and the crest speed. The performance is illustrated by comparing simulations with experimental data for an irregular breaking wave with a peak period of $12 \mathrm{~s}$ above deep water and for a bathymetry induced periodic wave plunging breaker over a trapezoidal bar. The comparisons show that the higher order models perform quite well; the extension with the breaking wave mechanism improves the simulations significantly.
\end{abstract}

\footnotetext{
${ }^{1}$ Published as:

R. Kurnia and E. van Groesen. High order Hamiltonian water wave models with wave-breaking mechanism. Coast. Eng., 93:55-70, 2014.
} 


\subsection{Introduction}

Accurate simulations of waves in deep water and in the coastal zone are important for various offshore activities and environmental issues. Efficiency and safety in design, installation and operations are most important for offshore wind farms, oil and gas platforms, ship and harbour design and for sustainable coastal management. Accurate wave models are needed to predict and describe waves also in extreme cases. This paper aims to contribute in presenting good and efficient models that are capable to describe rough waves and breaking waves.

The paper starts with the derivation of higher order Boussinesq-type models with exact dispersion by using the basic variational formulation of incompressible, irrotational surface waves with free surface under the influence of gravity. Nonlinear gravity waves have been the object study in many theoretical, numerical and experimental investigations. Boussinesa [1872] simplified the Euler equations for irrotational, incompressible fluid by approximating the Laplace equation for the interior fluid potential to obtain equations in horizontal quantities only by approximating the depth dependence. This leads to bi-directional and dispersive dynamic equations for the surface elevation and a fluid velocity. Korteweg and de Vries [1895] $(\mathrm{KdV})$ derived special solutions, the soliton and periodic equivalents, of a simplified Boussinesq equation for which the velocity variable is related to the surface elevation in such a way that the two dynamic equations lead to one unidirectional dynamic equation.

Zakharov [1968 formulated the basic Hamiltonian formulation of water waves on the surface of an infinitely deep fluid. The Hamiltonian in this formulation contains the kinetic energy, which is the Dirichlet integral of the fluid potential, that has to be expressed for given surface elevation in the other canonical variable which is the fluid potential at the surface. Craig and Sulem 1993 approximated Zakharov's formulation up to fifth order accuracy by a Taylor expansion of the Dirichlet-to-Neumann operator that maps the fluid potential at the fluid surface to the normal derivative of fluid potential at the surface. Recent KdV-type of models for waves above finite or infinite depth, called AB equations, have been developed by van Groesen and Andonowati [2007]. Using a second order Taylor expansion for the surface potential and for the vertical velocity around the still water level, leads to an approximation with exact dispersion in first and second order terms.

In this paper we present a simple derivation of higher order Hamiltonian equations for bi-directional waves. Just as in van Groesen and Andonowati [2007] an expansion around the still water level will be used to approximate the exact kinetic energy to any desired order in the wave amplitude, keeping the exact dispersion properties. To that end, the Taylor approximation of the normal velocity in the still water potential is expressed in the desired potential at the free surface after inverting the expansion of the free surface potential in the still water potential. The dynamic equations are then obtained by taking variations of the Hamiltonian which is explicitly expressed in the canonical variables. By invoking a uni-directionalization assumption, higher order $\mathrm{KdV}$ equations can be obtained as extensions of the $\mathrm{AB}$ equations; therefore the higher order Hamiltonian equations will be called AB Hamiltonian Systems (ABHS). 
Following the basic ideas used for other type of Boussinesq equations, we will extend the ABHS equations with a mechanism to deal with breaking waves. To that end, a trigger mechanism for the initiation of the wave breaking, and an energy dissipation mechanism have to be chosen.

Three dominant types of dissipation models in the current literature are the surface roller model (Schäffer et al. 1993], Madsen et al. [1997]), the vorticity model (Svendsen et al. 1996); Veeramonv and Svendsen 2000|) and the eddy viscosity model (Heitner and Housner [1970]; Zelt [1991]; Kennedy et al. [2000]). For the initiation of the breaking, different methods have been described: the trigger mechanism based on the slope angle variation (Schäffer et al. [1993]), based on the normal speed of the free surface elevation exceeding some threshold value (Kennedv et al. [2000]), Relative Trough Froude Number (RTFN) (Okamoto and Basco [2006]) and recently the Breaking Celerity Index method that couples the criterion proposed by Kennedy and the RTFN (D'Alessandro and Tomasicchio [2008]).

In this paper, we will implement for the ABHS models an extension of the eddy viscosity breaking model of Kennedy et al. 2000]. The extension makes it possible to deal with fully dispersive waves and will be applicable not only in shallow water but also in deep water. Besides that, we will investigate two variants for the viscosity coefficient. In one variant the decay is determined by the normal velocity as in Kennedy et al. 2000], while in a second variant the decay is determined by the tangential velocity; both variants will lead to almost similar results.

As trigger mechanism, we use the kinematic breaking criterion that the wave will break when the horizontal particle speed exceeds (a fraction of) the crest speed. The crest speed will be determined by an explicit expression of the local wavenumber as suggested by Stansell and MacFarlane 2002] by applying the spatial Hilbert transform; this mechanism will shown to be quite robust and applicable for all water depths.

The organization of the paper is as follows. In section 2.2 we present the variational description of surface waves and the consistent approximation of the ABHS equations up to fourth order. Section 2.3 deals with the extension to wave breaking with the eddy viscosity model and the kinematic breaking criterion. The numerical implementation with a pseudo-spectral code is briefly described in section 2.4. In section 2.5 we show results of simulations and compare these with accurate data. Experiments of deep water, irregular, breaking waves are available from the hydrodynamic laboratory MARIN (Maritime Research Institute Netherlands). Bathymetry induced breaking is compared with experiments of periodic long waves plunging breaking over a bar by Beji and Batties 1993. Conclusions and remarks will finish the paper.

\subsection{Variational wave description}

In section 2.2.1 we start with the description of the Hamiltonian formulation for surface water waves. In the expression of the kinetic energy functional appears the vertical velocity implicitly defined as operator linear in the surface potential and 
nonlinear in the elevation. In section 2.2 .2 we approximate the kinetic energy by using Taylor expansion with the potential at the still water level as intermediate variable. The approximation of the kinetic energy in second to fifth order leads to approximations of the dynamic equations with first to fourth order accuracy. We will verify that the Hamiltonian with the approximate kinetic energy leads to the same results as an approximation of the exact equations; as one consequence of this Hamiltonian consistent modelling, exact conservation of the approximate energy is guaranteed. In section 2.2.3 we describe a Hybrid Spatial Spectral method to deal with varying bottom in the application in section 2.5 .2

\subsubsection{Hamiltonian formulation}

Zakharov [1968], and later independently Broer [1974], showed that waves in one horizontal direction $x$ on the surface of an incompressible, inviscid fluid under the influence of gravity can be described by a set of Hamilton equations for the surface elevation $\eta(x, t)$ and the surface fluid potential $\phi(x, t)$ as canonical variables. Miles 1977. showed that this could have been derived from a variational pressure principle as formulated by Luke [1967] that could easily be rewritten as an action functional

$$
\int\left[\int \phi \partial_{t} \eta d x-\mathcal{H}(\phi, \eta)\right] d t
$$

for which the critical points, just as in Classical Mechanics, satisfy the Hamilton equations:

$$
\begin{aligned}
& \partial_{t} \eta=\delta_{\phi} \mathcal{H}(\phi, \eta) \\
& \partial_{t} \phi=-\delta_{\eta} \mathcal{H}(\phi, \eta) .
\end{aligned}
$$

Here we use the notation $\delta_{\phi}$ and $\delta_{\eta}$ to denote the variational derivative with respect to $\phi$ and $\eta$ respectively. The Hamiltonian is the total energy, the sum of potential and kinetic energy:

$$
\mathcal{H}(\phi, \eta)=\frac{1}{2} \int g \eta^{2} d x+\mathcal{K}(\phi, \eta)
$$

where the kinetic energy is formally given for finite and infinite depth, by

$$
\mathcal{K}(\eta, \phi)=\frac{1}{2} \iint|\nabla \Phi|^{2} d x d z
$$

Here $\Phi$ is the fluid potential that satisfies the Laplace equation in the interior fluid domain (representing the incompressibility condition for irrotational fluid motion), the surface condition $\Phi=\phi$ at $z=\eta$ and the impermeable bottom boundary condition. By applying Green's theorem, the kinetic energy can be expressed as

$$
\mathcal{K}(\eta, \phi)=\frac{1}{2} \int \phi \partial_{\mathbf{n}} \Phi d x
$$

With $W$ the vertical velocity $W=\Phi_{z}(x, \eta)$, here $\partial_{\mathbf{n}} \Phi=W-\eta_{x} \Phi_{x}$ at $z=\eta$ is the normal velocity at the surface, the Dirichlet-to-Neumann operator. Since 
$\phi_{x}=\Phi_{x}(x, \eta)+\eta_{x} W$, we get $\partial_{\mathbf{n}} \Phi=W\left(1+\eta_{x}^{2}\right)-\eta_{x} \phi_{x}$ and the kinetic energy can be rewritten as

$$
\mathcal{K}(\phi, \eta)=\frac{1}{2} \int \phi\left\{W\left(1+\eta_{x}^{2}\right)-\eta_{x} \phi_{x}\right\} d x
$$

It holds that $\delta_{\phi} \mathcal{K}=\partial_{\mathbf{n}} \Phi$, as shown by Zakharov [1968], and we get for the first Hamilton equation $\partial_{t} \eta=\delta_{\phi} \mathcal{H}(\phi, \eta)=W\left(1+\eta_{x}^{2}\right)-\phi_{x} \eta_{x}$. This is the kinematic surface condition, the continuity equation.

For variations of the Hamiltonian with respect to $\eta$, in the variation of kinetic energy it is important to realise that $\phi$ actually depends on $\eta$ since $\phi$ is the potential at the surface. Hence, for given variation $\delta \eta$, to keep $\phi$ fixed at the varied surface, we also get a contribution from the induced change $\delta \phi=W \delta \eta$. To compensate this, in taking variations of $\eta$ at fixed $\phi$ we have

$$
\left\langle\delta_{\eta} \mathcal{K}, \delta \eta\right\rangle=\mathcal{K}\left(\eta+\delta \eta, \phi-W \delta_{\eta}\right)=\left\langle\bar{\delta}_{\eta} \mathcal{K}, \delta \eta\right\rangle-\left\langle\delta_{\phi} \mathcal{K}, W \delta \eta\right\rangle
$$

where we denote by $\bar{\delta}_{\eta}$ the total variation with respect to $\eta$, allowing $\phi$ to change. Since

$$
\bar{\delta}_{\eta} \mathcal{K}=\frac{1}{2}|\nabla \Phi|_{z=\eta}^{2}=\frac{1}{2}\left[\left(\phi_{x}-\eta_{x} W\right)^{2}+W^{2}\right]
$$

we get in total

$$
\delta_{\eta} \mathcal{K}=\frac{1}{2}\left[\left(\phi_{x}-\eta_{x} W\right)^{2}+W^{2}\right]-W \partial_{\mathbf{n}} \Phi=\frac{1}{2}\left[\phi_{x}^{2}-W^{2}\left(1+\eta_{x}^{2}\right)\right] .
$$

This leads to the dynamic equation at the free surface $\partial_{t} \phi=-g \eta-\frac{1}{2} \phi_{x}^{2}+\frac{1}{2} W^{2}\left(1+\eta_{x}^{2}\right)$, which can be rewritten as $\partial_{t} \Phi+\frac{1}{2}|\nabla \Phi|^{2}+g \eta=0$ at $z=\eta$, which is the familiar Bernoulli equation.

For later reference we assemble the two Hamilton equations

$$
\begin{aligned}
& \partial_{t} \eta=W\left(1+\eta_{x}^{2}\right)-\phi_{x} \eta_{x} \\
& \partial_{t} \phi=-g \eta-\frac{1}{2} \phi_{x}^{2}+\frac{1}{2} W^{2}\left(1+\eta_{x}^{2}\right)
\end{aligned}
$$

\subsubsection{Consistent approximations}

The Laplace problem with prescribed potential $\phi_{0}$ at the still water level $z=0$ above a flat bottom can be solved explicitly using spatial Fourier transformation; in particular we can find explicitly the vertical velocity at the still water level which will be denoted by $W_{0}$. The result can be written using pseudo-differential operators. To that end we introduce the phase velocity operator $C_{0}$ which has symbol $\hat{C}_{0}=$ $\sqrt{g \tanh (k h) / k}$ in which $h$ is the depth and $k$ the wavenumber corresponding to $x$. Then $W_{0}=\partial_{z} \Phi(x, 0)$ is a linear operator in the surface potential $\phi_{0}$ given by

$$
W_{0}=-\frac{1}{g} \partial_{x} C_{0}^{2} \partial_{x} \phi_{0}=L \phi_{0}
$$

where we define $L=-\frac{1}{g} \partial_{x} C_{0}^{2} \partial_{x}$. Note that since the symbols of $C_{0}$ and $L$ are real and symmetric in the wavenumber, both these operators are real and symmetric. (Although the operators $C_{0}$ and $\partial_{x}$ commute, we prefer to use the notation $\partial_{x} C_{0}^{2} \partial_{x}$ 
to express the symmetry, and to emphasise that when using the tangential velocity $u=\partial_{x} \phi$ later on, it holds $L \phi=-\frac{1}{g} \partial_{x} C_{0}^{2} u$. )

The restriction to the still water level is sufficient to obtain the linearized equations. The kinetic energy is

$$
\mathcal{K}_{0}=\frac{1}{2} \int \phi_{0} . W_{0} d x=\frac{1}{2} \int \phi_{0} \cdot L \phi_{0} d x
$$

and the Hamilton equations read

$$
\begin{gathered}
\partial_{t} \eta=\delta_{\phi_{0}} \mathcal{H}\left(\phi_{0}, \eta\right)=W_{0} \\
\partial_{t} \phi_{0}=-\delta_{\eta} \mathcal{H}(\phi, \eta)=-g \eta
\end{gathered}
$$

Note that upon eliminating $\phi_{0}$ we obtain $\partial_{t}^{2} \eta=g L_{0} \eta$, which leads to the dispersion relation for harmonic modes $\omega^{2}=k^{2} \hat{C}_{0}^{2}(k)$, the exact relation for infinitesimal surface waves.

In order to obtain an expression for the kinetic energy as the quadratic functional of $\phi$ that depends on the finite surface elevation, we will use Taylor expansions. First we derive the expression for $\phi$ and $W$ around the still water level:

$$
\begin{gathered}
\phi=\Phi(x, \eta)=\Phi(x, 0)+\Phi_{z}(x, 0) \eta+\frac{1}{2} \Phi_{z z}(x, 0) \eta^{2}+\cdots \\
W=\Phi_{z}(x, \eta)=\Phi_{z}(x, 0)+\Phi_{z z}(x, 0) \eta+\frac{1}{2} \Phi_{z z z}(x, 0) \eta^{2}+\cdots
\end{gathered}
$$

Since $\Phi$ has to satisfy the Laplace equation, we can replace second derivative wrt $z$ of $\Phi$ by second derivatives wrt $x$ of $-\Phi$, so that $\phi=\phi_{0}(x)+\Phi_{z}(x, 0) \eta-\frac{1}{2} \Phi_{x x}(x, 0) \eta^{2}+$ $\ldots$ and $W=\Phi_{z}(x, 0)-\Phi_{x x}(x, 0) \eta-\frac{1}{2} \Phi_{x x z}(x, 0) \eta^{2}+\ldots$, which can be rewritten to third order of powers of $\eta$ in terms of $\phi_{0}$ and $W_{0}=L \phi_{0}$ as:

$$
\begin{aligned}
\phi(x) & =\left(1+\eta L-\frac{1}{2} \eta^{2} \partial_{x}^{2}-\frac{1}{3 !} \eta^{3} \partial_{x}^{2} L+\cdots\right) \phi_{0} \\
W(x) & =\left(L-\eta \partial_{x}^{2}-\frac{1}{2} \eta^{2} \partial_{x}^{2} L+\frac{1}{3 !} \eta^{3} \partial_{x}^{4}+\cdots\right) \phi_{0}
\end{aligned}
$$

Note that the $x$-derivatives do not act on the elevation. In order to express the expansion of $W$ in terms of $\phi$, we write $\phi=(1+A) \phi_{0}$ and approximate the inverse relation between $\phi$ and $\phi_{0}$ using $(1+A)^{-1}=\left(1-A+A^{2}-A^{3}+\cdots\right)$ and get (taking care of the order of the operators)

$$
\phi_{0}=\left(\begin{array}{c}
1-\eta L+\frac{1}{2} \eta^{2} \partial_{x}^{2}+\eta L \eta L \\
+\frac{1}{3 !} \eta^{3} \partial_{x}^{2} L-\frac{1}{2} \eta^{2} \partial_{x}^{2} \eta L-\frac{1}{2} \eta L \eta^{2} \partial_{x}^{2}-\eta L \eta L \eta L+\cdots
\end{array}\right) \phi
$$

Using this in the expression for $W$ in (Eq. 2.6) leads to the desired expression of $W$ 
in $\phi$, explicitly given for terms up to third order in $\eta$ :

$$
\begin{aligned}
W & =W_{0}+W_{1}+W_{2}+W_{3}+\cdots, \text { with } \\
W_{0} & =L \phi, \quad W_{1}=\left(-\eta \partial_{x}^{2}-L \eta L\right) \phi \\
W_{2} & =\left(L \eta L \eta L+\eta \partial_{x}^{2} \eta L+\frac{1}{2}\left(L \eta^{2} \partial_{x}^{2}-\eta^{2} \partial_{x}^{2} L\right)\right) \phi \\
W_{3} & =\left(\begin{array}{c}
\frac{1}{3 !} \eta^{3} \partial_{x}^{4}+\frac{1}{2} \eta^{2} \partial_{x}^{2} L \eta L-\frac{1}{2} \eta \partial_{x}^{2} \eta^{2} \partial_{x}^{2}-\eta \partial_{x}^{2} \eta L \eta L \\
-\frac{1}{2} L \eta^{2} \partial_{x}^{2} \eta L-\frac{1}{2} L \eta L \eta^{2} \partial_{x}^{2}-L \eta L \eta L \eta L+\frac{1}{3 !} L \eta^{3} \partial_{x}^{2} L
\end{array}\right) \phi
\end{aligned}
$$

Inserting the expansion in the kinetic energy, after some tedious but straightforward manipulations with partial integrations, we obtain the energy as an explicit functional in $\phi$ and $\eta$, for any desired order, given by

$$
\begin{aligned}
\mathcal{K}_{\text {exp }}(\phi, \eta) & =\frac{1}{2} \int \phi\left\{W\left(1+\eta_{x}^{2}\right)-\eta_{x} \phi_{x}\right\} d x \\
& =\mathcal{K}_{2}+\mathcal{K}_{3}+\mathcal{K}_{4}+\mathcal{K}_{5}+\cdots
\end{aligned}
$$

in which

$$
\begin{aligned}
\mathcal{K}_{2} & =\frac{1}{2} \int \phi W_{0} d x=\frac{1}{2} \int \phi L \phi d x \\
\mathcal{K}_{3} & =\frac{1}{2} \int \phi\left\{W_{1}-\phi_{x} \eta_{x}\right\} d x=\frac{1}{2} \int \phi M_{1} \phi d x \\
\mathcal{K}_{4} & =\frac{1}{2} \int \phi\left\{W_{2}+W_{0} \eta_{x}^{2}\right\} d x=\frac{1}{2} \int \phi M_{2} \phi d x \\
\mathcal{K}_{5} & =\frac{1}{2} \int \phi\left\{W_{3}+W_{1} \eta_{x}^{2}\right\} d x=\frac{1}{2} \int \phi M_{3} \phi d x
\end{aligned}
$$

with the symmetric operator $M_{n}$ given by

$$
\begin{aligned}
& M_{1}=\left[-L \eta L-\partial_{x}\left(\eta \partial_{x}\right)\right] \quad M_{2}=\left[L \eta L \eta L+\frac{1}{2}\left(\partial_{x}^{2}\left(\eta^{2} L\right)+L\left(\eta^{2} \partial_{x}^{2}\right)\right)\right] \\
& M_{3}=\left[\begin{array}{l}
-\frac{1}{3} \partial_{x}^{2}\left(\eta^{3} \partial_{x}^{2}\right)-L \eta L \eta L \eta L-\frac{1}{2} L \eta L\left(\eta^{2} \partial_{x}^{2}\right) \\
-\frac{1}{2} \partial_{x}^{2}\left(\eta^{2} L \eta L\right)-\frac{1}{3} L \partial_{x}\left(\eta^{3} \partial_{x} L\right)-\frac{1}{2} L\left(\eta^{2}\left(\partial_{x}^{2} \eta\right) L\right)
\end{array}\right] .
\end{aligned}
$$

The dynamic equations are obtained by taking the variational derivative of the Hamiltonian with respect to $\eta$ and $\phi$, and the result can be written as

$$
\begin{aligned}
& \partial_{t} \eta=\delta_{\phi} \mathcal{H}(\phi, \eta)=\left(L+M_{1}+M_{2}+M_{3}\right) \phi \\
& \partial_{t} \phi=-\delta_{\eta} \mathcal{H}(\phi, \eta) \\
& =-\left\{\begin{array}{c}
g \eta+\left[\frac{1}{2}\left(\left(\partial_{x} \phi\right)^{2}-(L \phi)^{2}\right)\right]+\left[L \phi \cdot\left(\eta \partial_{x}^{2} \phi+L \eta L \phi\right)\right] \\
+\left[\begin{array}{l}
-\frac{1}{2} \eta^{2}\left(\partial_{x}^{2} \phi\right)^{2}-\frac{1}{2}(L \eta L \phi)^{2}-L \eta L \eta L \phi \cdot(L \phi) \\
-\eta\left(\partial_{x}^{2} \phi\right) \cdot L \eta L \phi-\frac{1}{2} L\left(\eta^{2} \partial_{x}^{2} \phi\right) \cdot L \phi+\frac{1}{2} \eta^{2}\left(\partial_{x} L \phi\right)^{2} \\
-\frac{1}{2} \eta \partial_{x}^{2} \eta \cdot(L \phi)^{2}-\frac{1}{4} \partial_{x}^{2}\left(\eta^{2}(L \phi)^{2}\right)
\end{array}\right]
\end{array}\right\}
\end{aligned}
$$

Terms of the same order in $\eta$ and $\phi_{x}$ have been grouped together in square brackets here. It can be verified that this result is the same as an expansion of the right hand 
side of the exact dynamic equations (Eq. 2.4). The approximate equations have inherited the Hamiltonian structure, with exact conservation of the positive definite approximate energy as a consequence.

We finish with a few remarks and extensions.

The method described above is an extension of the second order uni-directional AB-equation (van Groesen and Andonowati [2007]) to bi-directional equations of arbitrary order in surface elevation $(\eta)$ and fluid potential $(\phi)$ at the free surface. To show this, consider the second order Hamiltonian equations, obtained from the Hamiltonian with terms up to third order

$$
\begin{aligned}
\mathcal{H}(\phi, \eta) & =\frac{1}{2} \int g \eta^{2}+\phi\left\{\left(W_{0}+W_{1}\right)-\eta_{x} \phi_{x}\right\} d x \\
& =\frac{1}{2} \int g \eta^{2}+\phi L \phi+\eta\left\{\phi_{x}^{2}-(L \phi)^{2}\right\} d x .
\end{aligned}
$$

Restriction to uni-directional waves to derive the $\mathrm{AB}$ equation, the dynamic equation $\partial_{t} \phi=-g \eta$ is combined with the equation for uni-directional propagation $\partial_{t} \phi=-A \phi$ where $A=C_{0} \partial_{x}$ to get $\phi=g A^{-1} \eta$. Restricting the action functional (Eq. 2.1) by substituting the relation $\phi=g A^{-1} \eta$, there results

$$
\mathcal{A}(\eta)=\int\left[\int g A^{-1} \eta . \partial_{t} \eta d x-\mathcal{H}\left(g A^{-1} \eta, \eta\right)\right] d t .
$$

The equation for critical points of this restriction $\mathcal{A}(\eta)$ leads to the equation

$$
\partial_{t} \eta=-A \delta_{\eta} \frac{1}{2 g} \mathcal{H}\left(g A^{-1} \eta, \eta\right) .
$$

Since $A=C_{0} \partial_{x}$ is a skew-symmetric operator, this equation has a Hamiltonian structure. Writing

$$
\partial_{t} \eta=-A \delta_{\eta} \mathcal{H}_{A B}(\eta), \text { with } \mathcal{H}_{A B}(\eta)=\frac{1}{2 g} \mathcal{H}\left(g A^{-1} \eta, \eta\right)
$$

and introducing the symmetric operator $B=C_{0}^{-1}$, the Hamiltonian $\mathcal{H}_{A B}$ can be written as in van Groesen and Andonowati [2007]

$$
\mathcal{H}_{A B}=\frac{1}{2} \int\left\{\eta^{2}+\frac{1}{2} \eta\left[g(B \eta)^{2}-\frac{1}{g}(A \eta)^{2}\right]\right\} d x .
$$

The AB equation has been extended for multi-directional waves to two horizontal directions (Lie and van Groesen [2010]) and for varying bottom (van Groesen and van der Kroon [2012]). In the same way the Hamiltonian systems above of any order can be extended for multi-directional waves above varying bottom.

\subsubsection{Hybrid Spatial Spectral implementation}

In order to deal with varying bottom, we use a Hybrid Spatial Spectral method to approximate the square phase speed operator $C_{0}^{2}(k, h)$. The method has been 
described in van Groesen and van der Kroon 2012] to approximate the dispersion relation above varying bottom.

The way to approximate the operator is by using a suitable smooth interpolation between the squared phase speed at the deep part $\left(h_{0}\right)$ and the shallow part $\left(h_{1}\right)$

$$
C_{\text {app }}^{2}(k, h(x))=a(x) C^{2}\left(k, h_{0}\right)+b(x) C^{2}\left(k, h_{1}\right)
$$

The coefficients $a(x), b(x)$ are determined using the value of a characteristic peak frequency, say $\omega=\nu$. Let $\kappa(x)$ be the wave number corresponding to this frequency at depth $x$

$$
\Omega(\kappa(x), h(x))=\nu .
$$

Then we require that the squared phase speed has the exact value for all these wave numbers

$$
C_{\text {exact }}^{2}(\kappa(x), h(x))=a(x) C_{\text {exact }}^{2}\left(\kappa(x), h_{0}\right)+b(x) C_{\text {exact }}^{2}\left(\kappa(x), h_{1}\right) .
$$

In addition we require that the frequency is also exact for these wave numbers:

$$
\Omega_{\text {exact }}(\kappa(x), h(x))=a(x) \Omega_{\text {exact }}\left(\kappa(x), h_{0}\right)+b(x) \Omega_{\text {exact }}\left(\kappa(x), h_{1}\right) .
$$

\subsection{Wave-breaking model}

To be able to simulate breaking waves in the ABHS models, we will describe in this section how to include an eddy-viscosity model for wave-breaking as an extension of the Hamilton equations. To that end, the eddy-viscosity model of Kennedy et al. [2000] will be modified in two ways. We start with the changes that are needed so that it becomes applicable for fully dispersive equations. In the second subsection we describe the way how the onset of breaking can be determined based on a kinematic criterion for the quotient of the fluid velocity at the crest and the crest velocity. In the last subsection we describe an alternative viscosity model.

\subsubsection{Eddy-viscosity model}

An eddy viscosity model is used to model turbulent mixing and dissipation caused by breaking. To achieve this, an eddy viscosity source term is added in the momentum equation, leaving the continuity equation unchanged. The source term should be chosen such that the momentum remains conserved while the energy will be dissipated.

The formulation is most naturally described using the elevation and velocity formulation of the equations. Hence we introduce the velocity $u$ as $u=\partial_{x} \phi$; this implies that $u$ is the tangential velocity at the surface. The Hamiltonian in the variables $\eta, u$ will be denoted by $\mathcal{H}(u, \eta)$. The variational derivative with respect to $\phi$ is then translated to the derivative with respect to $u$ according to $\delta_{\phi} \mathcal{H}(\phi, \eta)=$ $-\partial_{x} \delta_{u} \mathcal{H}(u, \eta)$. Upon adding an additional term $R$ to the momentum equation, the equations become:

$$
\begin{aligned}
& \partial_{t} \eta=-\partial_{x} \delta_{u} \mathcal{H}(u, \eta) \\
& \partial_{t} u=-\partial_{x} \delta_{\eta} \mathcal{H}(u, \eta)+R
\end{aligned}
$$


To derive an eddy-viscosity breaking model, the source term $R$ has to be chosen such that the momentum is not affected, in particular so that the momentum is constant if the bottom is flat. The momentum is defined by

$$
\mathcal{M}=\int H . u d x
$$

with $H=h+\eta$ the total depth. Physically this definition is plausible; this is confirmed by the fact that the Hamiltonian flow of this functional provides a pure translation, and therefore implies translation symmetry in the Hamilton equations above flat bottom:

$$
\begin{aligned}
& \partial_{t} \eta=-\partial_{x} \delta_{u} \mathcal{M}(u, \eta)=-\partial_{x} H=-\partial_{x} \eta \\
& \partial_{t} u=-\partial_{x} \delta_{\eta} \mathcal{M}(u, \eta)=-\partial_{x} u
\end{aligned}
$$

For the extended Hamiltonian system with source term $R$ we get for the time derivative of the momentum above flat bottom

$$
\frac{d}{d t} \mathcal{M}(u, \eta)=\int H R d x
$$

The requirement that the momentum should be conserved in the presence of $R$, we conclude that a general form for $R$ is as follows

$$
R=\frac{1}{H} \partial_{x} F
$$

where $\mathrm{F}$ is a 'flux' that should vanish for vanishing waves.

In the shallow water theory of Kennedy et al. [2000] the flux is taken to be $F=\nu . \partial_{x}(H u)$. Observe that this can be written in the shallow water model as $F=-\nu \cdot N$, where $N=\partial_{t} \eta$ is the normal velocity. This last expression can be used as a direct generalization to fully dispersive equations. Hence, with $N=-\partial_{x} \delta_{u} \mathcal{H}(u, \eta)$, we take

$$
F=-\nu . N \text { with } \nu=\beta H . N
$$

The so-called eddy viscosity coefficient $\nu$ contains the quantity $\beta$ which will determine the details of the breaking process, the initiation and cessation, and the decay. Here we will follow the approach of Kennedy et al. [2000] in which the process is described in terms of $N$. In the last subsection we will describe an alternative.

In the approach of Kennedy et al. 2000] the quantity $\beta$ depends on $N$, given by

$$
\beta=\delta_{B}^{2} \cdot B(N)
$$

Here $\delta_{B}$ is a mixing length coefficient; typically $\delta_{B}=1.2$, but it is stated that results are relatively insensitive to changes in this parameter for values in the interval $[0.9,1.5]$. The function $B$ depends smoothly on $N$ and will describe the breaking process between the start and cessation. $B$ vanishes for $N \leq N^{*}$ and varies monotonically from 0 to 1 to avoid an impulsive start of the breaking process that may 
lead to instability. A specific example is given by

$$
B(N)=\left\{\begin{array}{lll}
0 & \text { for } \quad & N \leq N^{*} \\
\frac{N}{N^{*}}-1 & & N^{*}<N<2 N^{*} \\
1 & & N \geq 2 N^{*}
\end{array}\right.
$$

The parameter $N^{*}$ will actually be taken a function of time: $N^{*}$ decreases over time from some initial value $N^{I}$ to a terminal quantity $N^{F}$. Breaking starts once the normal velocity $N$ exceeds the initial threshold value, $N^{I}$. Using $N$ in the breaking process with parameter $N^{*}$ as threshold value for the onset and cessation of breaking ensures in a simple manner that the dissipation is concentrated on the front face of the wave, as in nature. As breaking develops, the wave will continue to break even if $N$ drops below $N^{I}$. A simple linear relation is adopted to model the evolution of $N^{*}$

$$
N^{*}=\left\{\begin{array}{lll}
N^{I}-\tau\left(N^{I}-N^{F}\right) & \text { for } \quad 0 \leq \tau<1 \\
N^{F} & \text { for } \quad \tau \geq 1
\end{array} \quad \text { with } \tau=\frac{t-t_{b}}{T^{*}} .\right.
$$

Here $t_{b}$ is the initiation time, $T^{*}$ the transition time and $\tau$ is the relative elapsed time of the breaking process. Kennedy et al. [2000] proposed $N^{I}=b \sqrt{g h}$ for the initiation, $N^{F}=d \sqrt{g h}$ for the terminal quantity and $T^{*}=5 \sqrt{h / g}$ for the transition time. The value of $b$ is taken in the interval $[0.35,0.65]$ and $d=0.15$. None of these quantity should be regarded as universal values, they were chosen to match results of breaking simulations with measurements.

\subsubsection{Kinematic breaking criterion}

In Kennedy et al. 2000] the onset and cessation of breaking are determined by the values $N^{I}$ and $N^{F}$ of the normal velocity $N$ that are both proportional to the speed $\sqrt{g h}$; this is the speed in the shallow water case, which is the largest speed of the longest waves in dispersive models. For dispersive models, this could be replaced by a criterion that is related to the phase speed of some characteristic or dominant wave component. Since the normal velocity vanishes at the crest and is maximal near a zero-crossing, any condition formulated in $N$ does not refer directly to the properties of the wave in the crest. This is different for a so-called kinematic breaking criterion.

With reference to laboratory investigations, the kinematics of breaking waves has been discussed with measurement generally conducted by means of ElectroMagnetic Current-meter (ECM), Acoustic Doppler Velocimeters (ADV), Acoustics Doppler Velocity Profiler (ADVP), Laser Doppler Velocimeters (LDV), Particle Image Velocimetry (PIV) and also Micropropellers. Researchers have to select the most appropriate instrument to use based on the desired sampling frequency, length scale and type of phenomena. In the last two decades, advances in measuring techniques have made possible accurate measurement of the internal velocity field (e.g Ting and Kirby 1994, Doering and Donelan 1997], Brunone and Tomasicchio [1997], Cox and Kobavashi [2000], Tomasicchio 2006]). In particular, many researchers (Perlin et al. 1996], Chang and Liu [1998], Melville and Matusov [2002], Banner and Peirson 2007], Kimmoun and Branger 
[2007], Tian et al. [2010], Perlin et al. [2013], Shemer 2013]) have adopted the PIV technique to investigate the kinematic criterion; their results show that a wave will break when at crest the ratio of horizontal fluid velocity, denoted by $U$, and the crest speed, denoted by $C$, lies in the range between 0.75 to 0.95 .

Stansell and MacFarlane 2002] examined kinematic criterion by assessing three definitions of the wave speeds, namely the phase speed based on linear wave theory, partial Hilbert transforms of measured surface elevation, and the local position of maximum surface elevation. They found that $U / C \geq 0.95$ for spilling breakers and $U / C \geq 0.81$ for plunging breakers. This suggest that $U / C \geq 1$ may only be a sufficient but not necessary condition for the onset of wave breaking. Based on these results, we will use as kinematic breaking criterion the condition $U / C \geq b$, where $b \in[0.7,1]$ is an empirical parameter which can be tuned to measurement data.

As stated by Banner and Peregrine [1993], applying the kinematic breaking criterion to an irregular wave is complicated by the difficulty in defining or measuring the phase speed of a wave that is not of permanent form. To overcome this problem, Stansell and MacFarlane 2002] proposed a specific definition of phase speed based on the partial Hilbert transform with respect to time of the surface elevation of a wave $\eta(x, t)$. Different from this reference, we use the partial Hilbert transform with respect to $x$ to determine the instantaneous or local of wave number $k(x, t)$. Then, using the linear dispersion relation, the crest speed is defined by

$$
C(x, t)=\sqrt{\frac{g \cdot \tanh (k(x, t) \cdot h)}{k(x, t)}} .
$$

In order to determine the local wave number, first we define the spatial Hilbert transform by

$$
\mathbb{H}[\eta(x, t)]=\frac{1}{\pi} P \int_{-\infty}^{\infty} \frac{\eta\left(x^{\prime}, t\right)}{\left(x-x^{\prime}\right)} d x^{\prime}
$$

where $P$ stands for the Cauchy principal value of the integral. Then the phase function $\theta(x, t)$ is given by

$$
\tan \theta(x, t)=\frac{\mathbb{H}[\eta(x, t)]}{\eta(x, t)}
$$

and the local wave-number by

$$
k(x, t) \equiv \frac{\partial}{\partial x} \theta(x, t)
$$

This can be directly expressed in the Hilbert transform by differentiating (Eq. 2.19) with respect to $x$

$$
\frac{\partial \theta}{\partial x}=\frac{\cos ^{2} \theta}{\eta^{2}}\left(\eta \frac{\partial}{\partial x} \mathbb{H}[\eta]-\mathbb{H}[\eta] \frac{\partial}{\partial x} \eta\right) .
$$

Using the relation $\cos ^{2} \theta=\eta^{2} /\left(\eta^{2}+\mathbb{H}^{2}[\eta]\right)$, the local wavenumber is given by

$$
k(x, t)=\frac{1}{\eta^{2}+\mathbb{H}^{2}[\eta]}\left(\eta \frac{\partial}{\partial x} \mathbb{H}[\eta]-\mathbb{H}[\eta] \frac{\partial}{\partial x} \eta\right) .
$$


Then the value of $N^{I}$ is determined by the maximum value of the normal velocity in the so-called breaking nodes when the kinematic criterion $U / C \geq b$ is satisfied in the crest:

$$
N^{I}=\max _{x_{c}<x<x_{t}} N\left(x, t_{b}\right)
$$

The breaking nodes are determined from the crest $\left(x_{c}\right)$ to the trough $\left(x_{t}\right)$ position of the front face wave by tracking the sign-change of the gradient of the wave profile. The breaking process between the start $\left(t_{b}\right)$ and cessation $\left(t_{f}\right)$ concentrate on the front face wave locally, so that $N(x, t)$ in Eq. 2.16 is restricted to $x \in\left[x_{c}, x_{t}\right]$ and $t \in\left[t_{b}, t_{f}\right]$. We use as terminal quantity the value $N^{F}=d . N^{I}$ with $d \approx 0.3$ as empirical value; for the transition time we take $T^{*}=\frac{T_{p}}{2}$, based on a suggestion by Schäffer et al. [1993] : $T^{*} \in\left[\frac{T_{p}}{10}, \frac{T_{p}}{2}\right]$, where $T_{p}$ is the peak period of the irregular wave.

In order to show the relation between the kinematic criterion and the critical slope condition, Fig. 2.1 present the results of a simulation described in section 2.5.1 Fig. 2.1 shows that with this approach, the value of $N^{I}$ for all breaking events lie in the range from $8 \mathrm{~m} / \mathrm{s}$ to $17 \mathrm{~m} / \mathrm{s}$ with the most frequent $10 \mathrm{~m} / \mathrm{s}$. The slope of the wave front $\left(\partial_{x} \eta\right)$ is calculated using the relation $\partial_{t} \eta+c \partial_{x} \eta=0$ with $c$ the wave celerity. For the peak wave number $\left(k_{\nu}\right)$ in the irregular wave simulation $\left(k_{\nu}=0.0298\right.$, depth $\left.=510 \mathrm{~m}\right)$ the phase speed is $c=18.15 \mathrm{~m} / \mathrm{s}$. Therefore the critical slope of the wave front is in between 0.45 and 0.94 with the most frequent is 0.55 , the critical angle is in between $23.8^{\circ}$ and $43.1^{\circ}$ with the most frequent $28.9^{\circ}$.

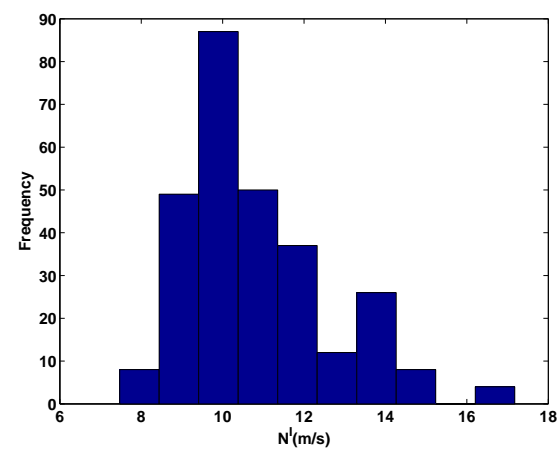

Figure 2.1: Histogram of the initial normal velocity $\left(N^{I}\right)$ for all breaking events during the ABHS 4break simulation with criterion $U / C>1$ for irregular breaking over a flat bottom. (See section 2.5.1)

\subsubsection{Alternative viscosity model}

The viscous decay of the wave as described above is determined by the decay in time of $N^{*}$ through $B(N)$. When using the kinematic breaking criterion above, and hence having available the quotient $U / C$, it seems more natural to let the decay be 
determined by the tangential velocity $(u)$. This leads to an alternative approach where $B(N)$ and $N^{*}$ are replaced by $B(u)$ and $u^{*}$ as

$$
B(u)=\left\{\begin{array}{lll}
0 & \text { for } \quad u \leq u^{*} \\
\frac{u}{u^{*}}-1 & & u^{*}<u<2 u^{*} \\
1 & & u \geq 2 u^{*}
\end{array}\right.
$$

with

$$
u^{*}=\left\{\begin{array}{ll}
u^{I}-\tau\left(u^{I}-u^{F}\right) & \text { for } \quad 0 \leq \tau<1 \\
u^{F} & \text { for } \tau>1
\end{array} \quad \text { with } \tau=\frac{t-t_{b}}{T^{*}} .\right.
$$

where $u^{I}=U$, when the kinematic criterion $U / C \geq b$ is satisfied in the crest. For the terminal quantity we take the value $u^{F}=d \cdot u^{I}$ with $d \approx 0.3$ and for the transition time $T^{*}=\frac{T_{\mathrm{peak}}}{2}$. Similar with the previous scheme, the breaking process is locally concentrated on the front face of the wave, so that $u(x, t)$ in Eq. 2.22 is restricted to $x \in\left[x_{c}, x_{t}\right]$ and $t \in\left[t_{b}, t_{f}\right]$.

The difference in the resulting dynamics for the two alternative viscosities is not easy to predict. The difference will show itself for instance in the decay of the Hamiltonian, which can be calculated by investigating the time derivative:

$$
\begin{aligned}
\frac{d}{d t} \mathcal{H}(u, \eta) & =\int R \cdot \delta_{u} \mathcal{H} d x=\int \partial_{x}^{-1} R \cdot\left(-\partial_{x} \delta_{u} \mathcal{H}\right) d x \\
& =\int \partial_{x}^{-1} R \cdot N d x=\int \partial_{x}^{-1}\left(\frac{1}{H} \partial_{x} F\right) \cdot N d x .
\end{aligned}
$$

For a more explicit expression we consider the approximation for deep water, so that $1 / H$ can be considered to be constant. Then

$$
\frac{d}{d t} \mathcal{H}(u, \eta) \approx \int \frac{1}{H} F \cdot N d x=-\int \beta N^{3} d x .
$$

Hence, the choices $\beta \sim B(u)$ or $\beta \sim B(N)$ will give somewhat different decays. In section 2.5.1 we will show that for a characteristic irregular wave breaking case the differences in energy decay and in the resulting wave profiles and wave signals are rather small.

\subsection{Numerical implementation}

For the simulations of waves above a flat bottom to be described in the next section, we use a pseudo-spectral implementation of the equations. The implementations were translated in Matlab codes, and the built-in Runge Kutta ODE45 solver with variable step size was used for time advancement. All variables in the following are dimensional, and the value of the gravitational acceleration was taken to be $g=9.81 \mathrm{~m} / \mathrm{s}^{2}$.

The lay-out of the equations for the numerical implementation contains various additional terms, and can be written as

$$
\begin{aligned}
& \partial_{t} \eta=R H S-\alpha C(k) \eta \cdot \chi_{\mathrm{damp}}+S(x, t) \\
& \partial_{t} u=R H S+R-\alpha C(k) u \cdot \chi_{\mathrm{damp}} .
\end{aligned}
$$


Here $R H S$ is the right hand side of the Hamiltonian equation of a specific order (Eq. 2.7). We briefly describe the various new terms.

\subsubsection{Damping zones}

In the spectral implementation, the right end of the spatial interval is coupled to the left by periodicity; to decouple these endpoints, to prevent 'spatial looping', we use a damping zone near the endpoints, similar as in van Groesen and van der Kroon 2012]. To that end we designed a smoothed characteristic function $\chi_{\text {damp }}$ that is non-vanishing only in an area near the endpoints of total x-interval. Then the terms $-\alpha C(k) \eta \chi_{\text {damp }}$ and $-\alpha C(k) u \chi_{\text {damp }}$ with $\alpha>0$ attenuate the waves in the damping zone, in an exponential way with a factor $\exp (-\alpha \ell)$ where $\ell$ is the length of the damping zone. For the simulation in this paper, $\alpha$ and $\ell$ are chosen such that attenuation is of the order $10^{-2}$ or less; specifically we took $\alpha=1$ and $\ell=7$.

\subsubsection{Nonlinear wave generation}

In order to generate waves that correspond to a measured time signal $s(t)$ of the surface elevation at a specific position $x_{0}$, we use a source term $S(x, t)$ as an embedded influx to generate to the right travelling waves with surface elevation $s(t)$ at $x_{0}$ as described in Lie et al. 2014]. The source term is not unique; if we restrict to sources of the form $S(x, t)=\gamma(x) f(t)$, the spatial-temporal Fourier transform $\bar{S}(k, \omega)$ is unique only if $k$ and $\omega$ are related by the dispersion relation. One possible choice (for $x_{0}=0$ ) would be $\bar{S}(k, \omega)=V_{g}(k) \check{s}(\omega)$, where $V_{g}$ is the group velocity and $\check{s}(\omega)$ the temporal Fourier transform of $s(t)$. However, on deep water, as in the example in the next section, the inverse Fourier transform $\gamma_{0}(x)$ of $V_{g}$ has a wide spatial extent in which the waves are not completely developed yet. Therefore we adjust the generation domain with influx position $X_{i n}=0$ by multiplying $\gamma_{0}(x)$ with a Gaussian function $\zeta(x)=\exp \left(-\left(x-X_{\text {in }}\right)^{2}\right) /\left(2 L_{\text {sup }}^{2}\right)$ with $L_{\text {sup }}=0.4 \lambda_{p}$, leading to the spatial generation function $\gamma(x)=\zeta(x) \gamma_{0}(x)$. Then the influx signal $f(t)$ has to be taken to be the inverse temporal Fourier transform of $\check{s}(\omega) . V_{g}(k(\omega)) / \hat{\gamma}(k(\omega))$ where $k(\omega)$ follows from the dispersion relation, and $\hat{\gamma}$ is the spatial Fourier transfor of $\gamma$. We use this source term in the simulations in the next section, with one additional adjustment.

The adjustment is needed when influxing very nonlinear waves; then the linear influxing in the nonlinear dynamic model may generate spurious modes. To prevent this problem, as described in Lie et al. 2014], we seperate the linear term from the nonlinear terms $R H S_{\text {nonlin }}$ in $R H S$ and multiply the nonlinear terms with a smoothing function $\chi_{\text {nonlin }}$. For $\chi_{\text {nonlin }}$ we choose

$$
\begin{aligned}
\chi_{\text {nonlin }}= & 1-\max \left\{\operatorname{sign}\left(R_{1}-x\right), \min \left[1+\operatorname{sign}\left(R_{1}+\Delta R-x\right),\right.\right. \\
& \left.\left.\left(\sin \left(\pi\left(x-R_{1}+\Delta R\right) /(2 \Delta R)\right)\right)^{2}\right]\right\}
\end{aligned}
$$

where $\Delta R=R_{2}-R_{1}, R_{1}=X_{i n}+0.1 \lambda_{p}, R_{2}=R_{1}+0.5 \lambda_{p}$ and $\lambda_{p}$ is the peak wavelength of the irregular wave. The plot of $\chi_{\text {nonlin }}$ is shown in Fig. 2.3 at the right. 


\subsection{Simulation results}

In this section we show the performance of the ABHS equations extended with the breaking model. We compare results of the simulations with measurements as performed at MARIN hydrodynamic laboratory and measurements were conducted by Beji and Batties 1993. The first subsection describes simulations of an irregular wave with peak period $12 \mathrm{~s}$ in deep water. Comparison with experiments are given for simulation with fourth order accuracy, without and with breaking. For the wave breaking process, two values of the kinematic breaking condition are used, and results for two different models of the viscosity are shown. The second subsection describes for periodic waves the bathymetry induced breaking over a bar Beji and Batties, 1993 ; we focus on the long wave $(0.4 \mathrm{~Hz})$ plunging breaking. Comparison with measurement of the ABHS simulations with second and third order accuracy with wave-breaking are given.

\subsubsection{Irregular wave breaking over a flat bottom}

\section{Geophysical layout and numerical settings}

The influx signal generated is an irregular wave in which breaking was observed. All the parameters to be given below correspond to a spatial scaling with a factor 50 of the wavetank measurement at MARIN hydrodynamic laboratory, measurement identification $223002 F$.

The wave above a depth of $h=510 \mathrm{~m}$ is an irregular wave with a spectrum of JONSWAP-type. The significant wave height is approximately $10.2 \mathrm{~m}$, the peak period $12 \mathrm{~s}$, and the corresponding peak wavelength $\lambda_{p}=211 \mathrm{~m}$; the initial steepness of the peak wave is $k_{p} . a=0.256$. In the experiment there are five positions where the wave height is measured: W1 at $x=0, \mathrm{~W} 2$ at $x=275.4 \mathrm{~m}, \mathrm{~W} 3$ at $x=312.4$ $\mathrm{m}, \mathrm{W} 4$ at $x=642.6 \mathrm{~m}$ and W5 at $x=1593.4 \mathrm{~m}$. The measured surface elevation at the first position W1 is used as influx signal for our simulation; taking the measured elevation avoids errors that may arise when translating the flap motion to a surface elevation.

The layout of the experimental set up is given in Fig. 2.2, In Fig. 2.3, the plots at the left show the time signal and the normalized spectrum at the influx position W1. At the right the spatial calculation domain is shown with the generation function $\gamma(x)$ centered at W1 that is used in the source function, and with the smooth characteristic functions for the damping zone and the nonlinear adaptation (Eq. 2.26).

Most results to be presented were obtained with simulations with grid size $d x=$ $2.63 \mathrm{~m}$ (taking $2^{11}$ modes in the numerical computation interval). This choice is motivated to prevent aliasing for fourth order models. Actually, for fourth order accuracy we want to be able to simulate frequencies correctly up to $4 \omega_{p}$, which corresponds to wavenumbers up to $\Omega^{-1}\left(4 \omega_{p}\right)$ where $\Omega^{-1}$ is inverse of the dispersion relation and $\omega_{p}$ is the peak frequency. To prevent aliasing in 4 th order, this would lead to $k_{\max }=8 \Omega^{-1}\left(4 \omega_{p}\right)$. Since the 4 th order nonbreaking codes cannot deal with this value, we relaxed it somewhat to $k_{\max }=6 \Omega^{-1}\left(4 \omega_{p}\right)$ which corresponds to $2^{11}$, 


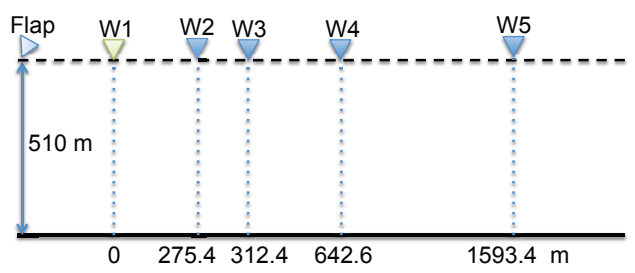

Figure 2.2: Layout of the experimental set-up. The flat bottom has depth $510 \mathrm{~m}$. The waves are generated by the wave flap at the left, and the elevation is measured at positions W1, W2, W3, W4, W5. The elevation signal at $W 1$ is used as influx for the simulations; the other measurements are used for comparison with simulation results.
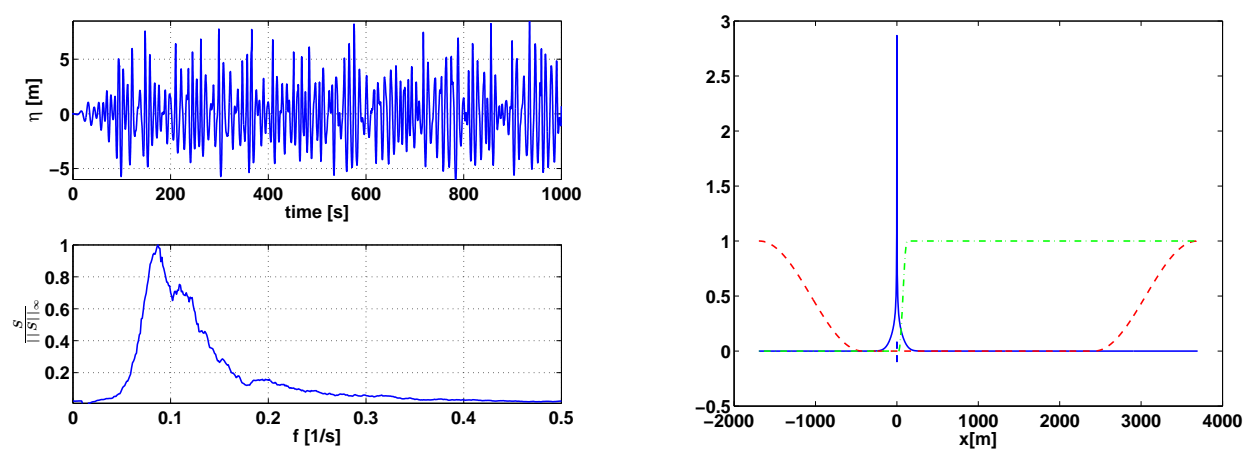

Figure 2.3: The plots at the left show the time signal and normalized spectrum at influx position W1. At the right the spatial calculation domain is shown with the generation function $\gamma(x)$ centered at W1 (blue, solid) used in the source function, and with the smooth characteristic functions for the damping zone (red, dashed) and the nonlinear adaptation (green, dashed-dot).

which was shown to be sufficient for most calculations. This leads to more than 80 points per wavelength at peak frequency and more than 16 points for the smallest wavelength. From simulations based on these considerations we observed that this choice of $d x$ gave in most cases the best results; smaller values of $d x$ may lead to too much high wavenumber generation, and for larger values the high frequency removal is too large and essential phenomena, such as high waves, are not well presented. For plotting purposes and to get higher precision of the distance between a grid point and the measurements positions, we interpolated the calculated results on a grid size of $d x / 2$.

We show simulation results for various models, for ABHS2, 3 and 4 and for ABHS4break which is ABHS4 with eddy viscosity breaking model.

In plots of elevation signals we compare the MARIN signals (solid, black) with signals of various simulation methods; the horizontal axis is in seconds $[\mathrm{s}]$, and the vertical axis is the elevation in meters [m]; note that each plot has different scale to show the results in the best possible way.

In plots of spectra, we split plot of measured and simulated spectra. The horizon- 
tal axis is the linear frequency in Hz: $f[1 / s]$. The plotted spectrum is the amplitude spectrum of the measured waves: the absolute value of the temporal Fourier transform of the signal, normalized by the maximum of the influx spectrum, with axis at the left. Using the amplitude spectrum, instead of the energy spectrum, gives a clearer representation of the high frequencies. Overlaying the spectrum plot various curves show the difference of the simulated spectrum with the measured spectrum, normalized by the maximal value of the influx spectrum, the values of these differences can be read from the axis at the right.

\section{Results}

We start the description of the performance of simulations with various models with some quantitative information. In Table 2.1. we show the correlations and the relative computation times. The correlation between the time traces of the measurement and the simulation is defined as the inner product between the normalized signals. Deviations from the maximal value 1 of the correlation measures especially the error in phase, a time shift of the simulation; for correlation -1 the simulation is in counter phase with the measurement. The length of the time interval over which the quantities are calculated is the full time trace $(1000 \mathrm{~s})$. The relative computation time is defined as the cpu-time divided by the total geotime of the simulation; the calculations were performed on a desktop computer with $\mathrm{CPU}$ i7, 3.4 GHz processor with 16 GB memory.

The performance as expressed by the correlation shows a consistent trend: higher order models perform better over all measurement positions; when breaking plays a role, at positions $\mathrm{W} 4$ and $\mathrm{W} 5$, the extension with the breaking method performs substantially better. Higher order and including breaking increases the computation time, as expected. For one breaking method we give in Table 2.2 also the variance quotient and the values of the significant wave height (approximated as 4 times the square root of the variance) for the simulation and the measurement.

Table 2.1: Correlations at measurement positions between simulations and measurements and the relative computation time for the various ABHS models.

\begin{tabular}{lccccc}
\hline & \multicolumn{4}{c}{ Correlation } & \\
& W2 & W3 & W4 & W5 & \\
\hline ABHS2 & 0.87 & 0.86 & 0.76 & 0.71 & 0.14 \\
ABHS3 & 0.92 & 0.91 & 0.89 & 0.77 & 0.21 \\
ABHS4 & 0.92 & 0.92 & 0.90 & 0.74 & 0.35 \\
ABHS4break $U / C \geq 0.8$ & 0.92 & 0.92 & 0.89 & 0.87 & 0.54 \\
ABHS4break $U / C \geq 1$ & 0.92 & 0.92 & 0.89 & 0.87 & 0.53 \\
\hline
\end{tabular}

We now describe the main results for the non-breaking model ABHS4, and the breaking model ABHS4break. In Fig. 2.4, we show the time signal at W2, W3, W4 and W5, at the top for the time interval $(100 ; 500) \mathrm{s}$ and at the bottom for the time interval $(600,1000)$. In Fig. 2.5 at the left, we show amplitude spectra of the full simulation time (1000) s. There are two simulations for ABHS4break, the difference being the value for the onset of breaking, one for the criterion $U / C \geq 1$ and 
Table 2.2: Specific information for model ABHS4break with $U / C \geq 1$.

\begin{tabular}{lcccr}
\hline & W2 & W3 & W4 & W5 \\
\hline Variance quotient & 0.99 & 0.96 & 0.89 & 1.01 \\
$H_{s}($ Simul,Meas $)$ & $(9.91,9.98)$ & $(9.8,10.01)$ & $(9.48,10.07)$ & $(8.52,8.45)$ \\
Correlation & 0.92 & 0.92 & 0.89 & 0.87 \\
\hline
\end{tabular}
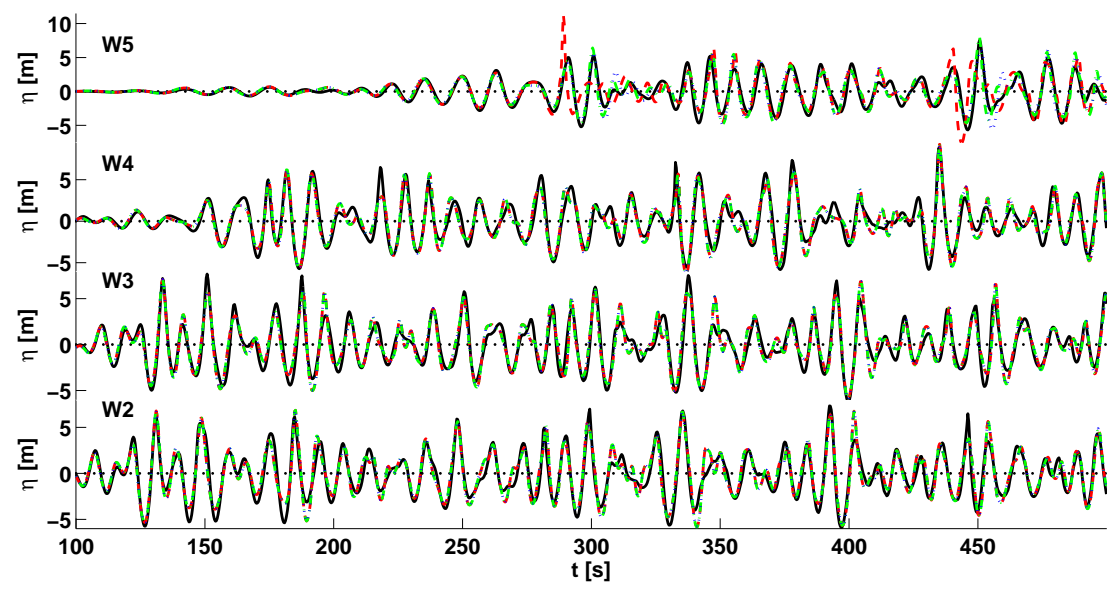

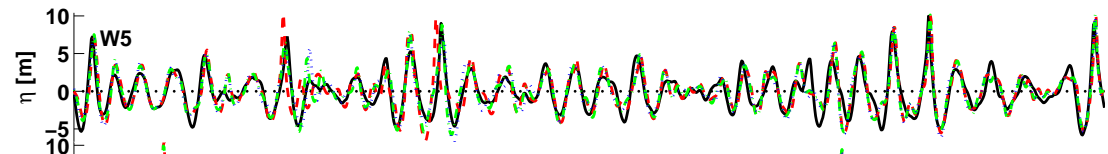

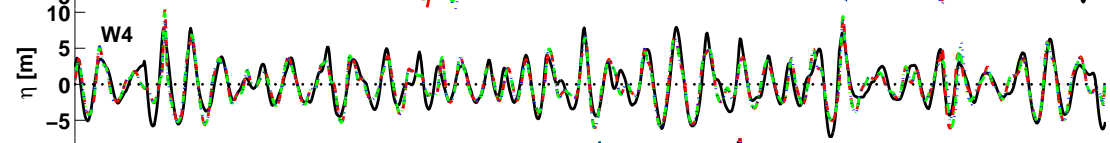

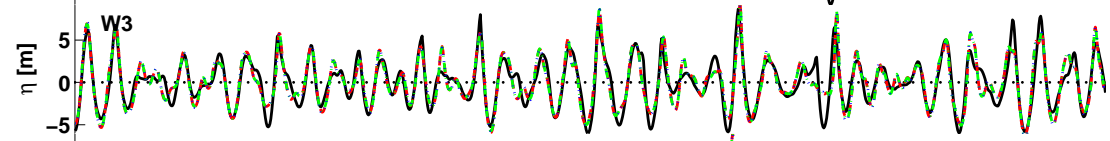

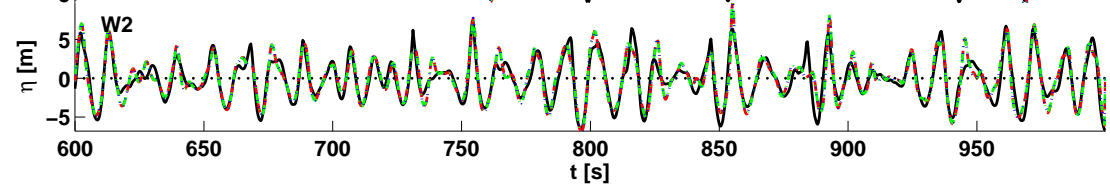

Figure 2.4: Elevation time traces at positions W2, $x=275.4 \mathrm{~m}, \mathrm{~W} 3, x=312.4 \mathrm{~m}$, $\mathrm{W}_{4}$, $x=642.6 \mathrm{~m}$ and $\mathrm{W5}, x=1593.4 \mathrm{~m}$ are shown for the measurement (black, solid) and for simulations with the non-breaking model ABHS4 (red, dashed), and the breaking models ABHS4break $U / C \geq 1$ (green, dashed-dot), and ABHS4break $U / C \geq 0.8$ (blue, dotted); in the top for the time interval $(100 ; 500) s$ and in the bottom for the interval $(600 ; 1000) s$.

one for $U / C \geq 0.8$. Differences between ABHS4 and ABHS4break are particularly noticeable in the time signal at W5 around $t=290 \mathrm{~s}, t=430 \mathrm{~s}, t=690 \mathrm{~s}$ and 

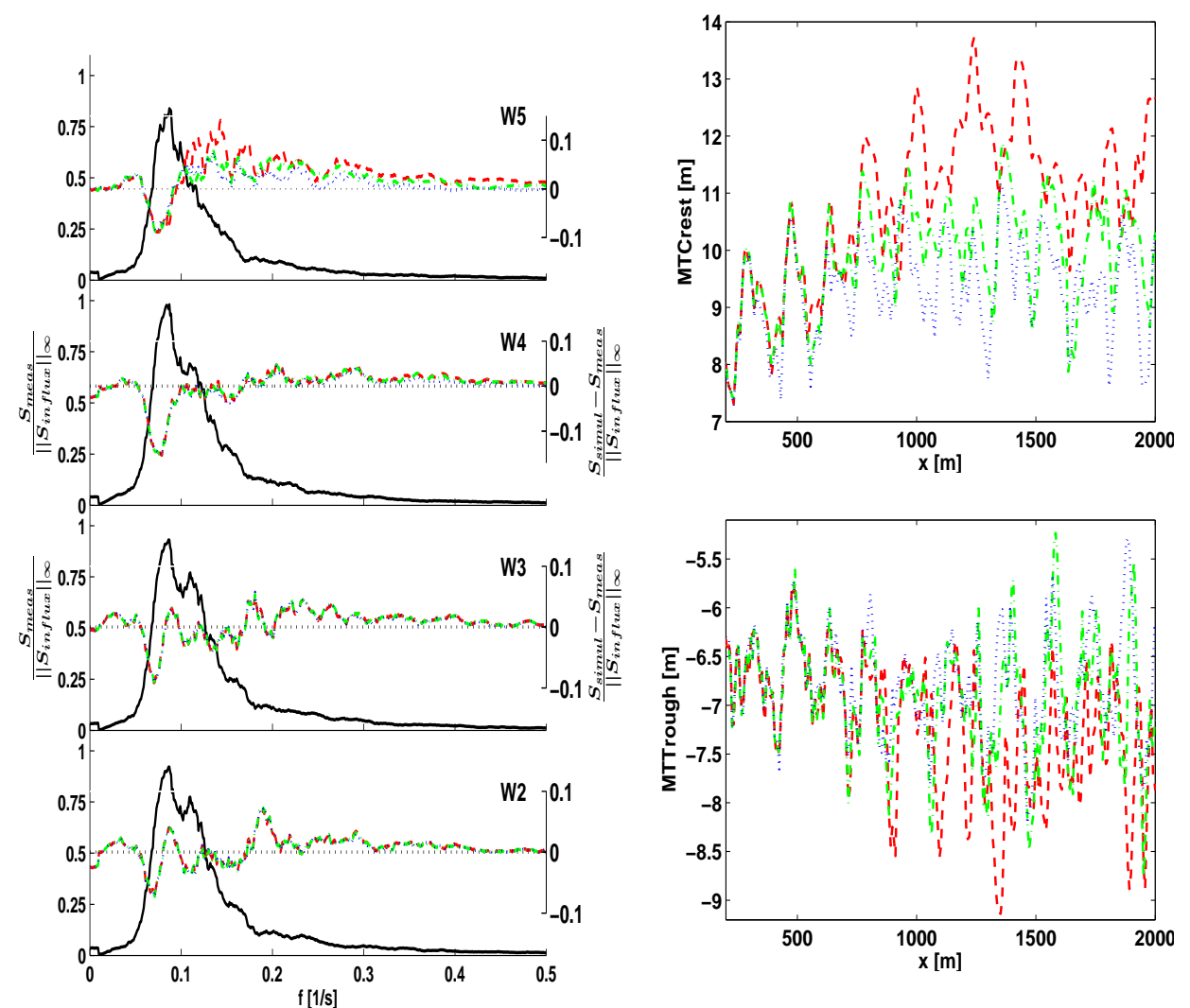

Figure 2.5: The left column shows the normalized amplitude spectra of the measurement (black, solid) at the four positions; the plots in the overlay show the differences for the simulations. In the right column, the plots show the Maximal Temporal Crest (MTC at the top) and Minimal Temporal Trough (MTT at the bottom) for different simulations. The simulations are ABHS4 (red, dashed), ABHS4break $U / C \geq 1$ (green,dashed-dot), and ABHS4break $U / C \geq 0.8$ (blue, dotted).

$t=720 \mathrm{~s}$; the high wave of ABHS4 is reduced substantially in ABHS4break by the viscous dissipation model, and the wave height and phase agree better with the measurement data. Also the spectra are improved for the breaking models for higher frequencies. The quantitative data in Table 2.1 show that ABHS4break has a higher correlation, an improvement with $16 \%, 10 \%$ and $13 \%$ compared to ABHS2, ABHS3 and ABHS4 respectively.

The Maximum Temporal Amplitudes in Fig. 2.5 at the right show that the expected decrease of extreme wave height due to wave-breaking is visible. ABHS4break with $U / C \geq 0.8$ is more dissipative than ABHS4break with $U / C \geq 1$, as expected. The maximal wave heights on these curves are now 18.7, 14.7 and $13.11 \mathrm{~m}$ for ABHS4, ABHS4break with $U / C \geq 1$ and ABHS4break with $U / C \geq 0.8$ respectively.

Fig. 2.6 shows the evolution of the maximum horizontal particle speed $(U)$ and the crest speed $(C)$ for ABSH4, ABHS4break $U / C \geq 1$ and ABHS4break $U / C \geq 0.8$. 
The times of breaking initiation for $U / C \geq 1$ are visible as the intersection points of the $U$ and $C$ curves. For the onset criterion $U / C \geq 0.8$ the breaking starts earlier.

The wave breaking leads to a decrease of the crest height at W5, and to a decrease of the energy as shown by the value of the significant wave height in Table 2.2. Fig. 2.7 shows the evolution of the spatial wave profiles during the breaking process which starts at position $953.3 \mathrm{~m}$ at time $221 \mathrm{~s}$ and continues for $6.27 \mathrm{~s}$; the reduction in wave height during breaking is clearly visible. This breaking event gives a reduction of wave height at W5 around $t=290 \mathrm{~s}$. The plot of the evolution of $U$ and $C$ in Fig. 2.6 shows that around $t=290 \mathrm{~s}$ there is no breaking event in the simulation with breaking model. Therefore the reduced wave height at W5 around $t=290 \mathrm{~s}$ is caused by the breaking events at position 824.25 at time $204.17 \mathrm{~s}$ which continues for $6.2 \mathrm{~s}$, at position $953.3 \mathrm{~m}$ at time $221 \mathrm{~s}$ for $6.4 \mathrm{~s}$ and at position $1120 \mathrm{~m}$ at time $242.05 \mathrm{~s}$ for $1.28 \mathrm{~s}$.

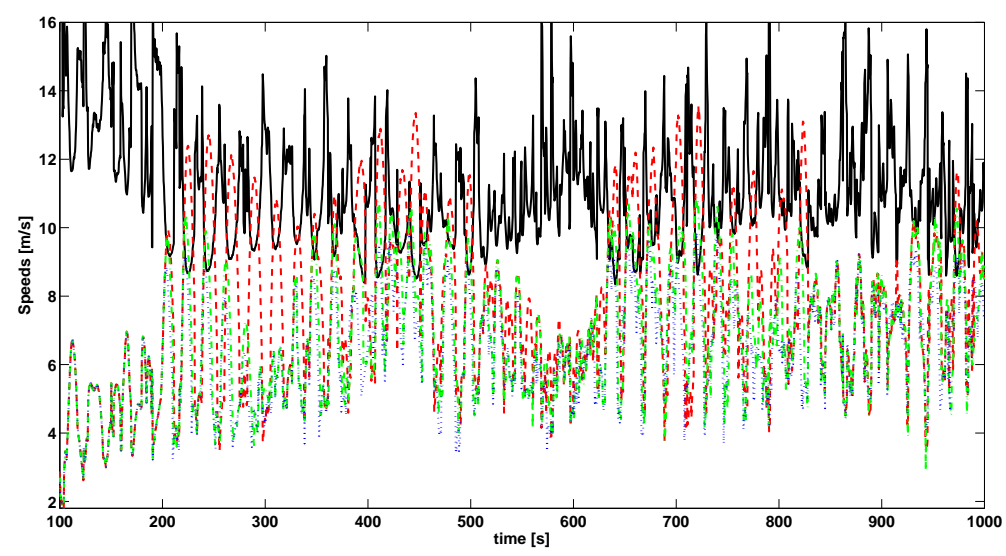

Figure 2.6: Plot of the crest speed (C) of ABHS4 (black, solid) and of the horizontal particle speed $(U)$ as calculated for 3 different models: ABHS4 (red, dashed), ABHS4break with $U / C \geq 1$ (green, dashed-dot) and ABHS4break with $U / C \geq 0.8$ (blue, dot).

\section{Simulation of alternative viscosity model}

In this subsection we show the comparison of ABHS4break simulations with the different viscosity model as described in subsection 2.3.3. both simulations use the same breaking criterion $U / C \geq 1$. In Fig. 2.8 and 2.9 at the left, we show time signals and spectra at W4 and W5. The differences between the simulations with the viscosity models for $\mathrm{B}(\mathrm{u})$ and $\mathrm{B}(\mathrm{N})$ are hardly visible, and the correlations with measurements are nearly the same as seen in Table 2.3. The Maximum Temporal Amplitude plots in Fig. 2.9 at the right show small differences, just as in Fig. 2.11 for the horizontal particle speed $(U)$ and the crest speed $(C)$.

The breaking process now starts at position $958(950.6) \mathrm{m}$, time $221.6(221) \mathrm{s}$ and continues for $9.8(6.2) \mathrm{s}$; the quantities in brackets refer to the values for $B(N)$. The plot of the evolution of $U$ and $C$ in Fig. 2.11 shows that the first breaking event occurs at time $204.17 \mathrm{~s}$ and position $824.25 \mathrm{~m}$ for both viscosity models $B(u)$ 

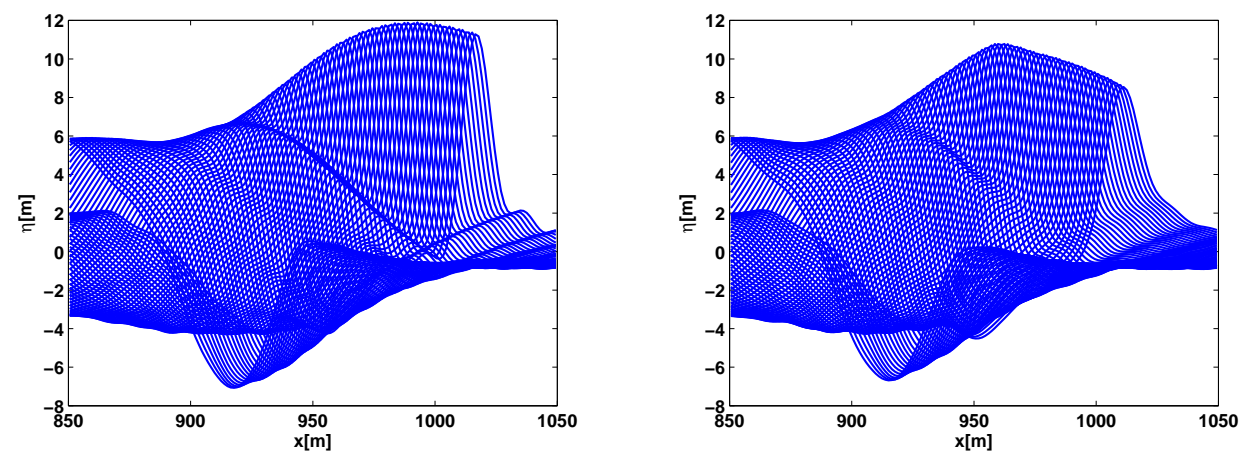

Figure 2.7: Calculated successive spatial wave profiles for times in the interval $(215 ; 226)$, at the left for ABHS4 without wave breaking, and at the right for $A B H S 4 b r e a k U / C \geq 1$. The breaking process starts at position $950.6 \mathrm{~m}$, time $221 \mathrm{~s}$ and the breaking continues for $6.4 \mathrm{~s}$.
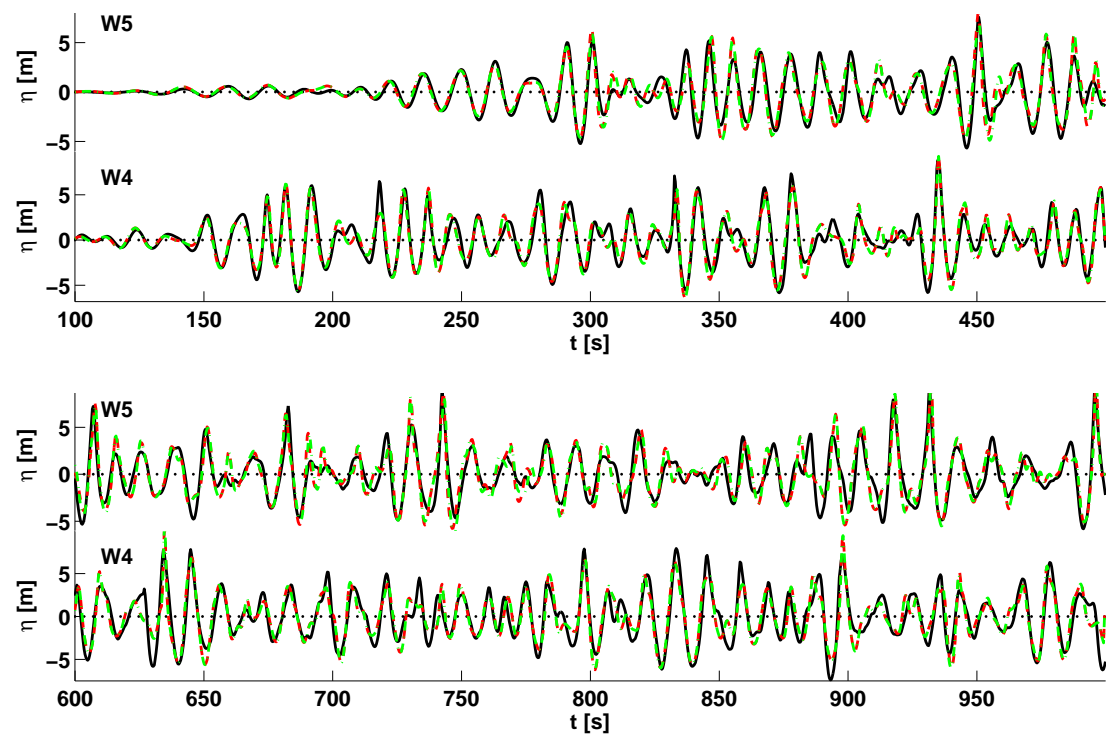

Figure 2.8: Same as Fig. 2.4, now for the breaking models ABHS4break with $B(N)$ (red, dashed), and ABHS4break with B(u) (green, dashed-dot).

and $B(N)$. However, after the first breaking, next breaking events occur at different position and time because of the different dissipation processes for the two different viscosity models. The longer breaking time for $B(u)$ leads to a somewhat smoother breaking process, as can be seen by comparing the evolution of the wave profiles during the breaking process with $B(u)$ in Fig. 2.10 with that for $B(N)$ in Fig. 2.7.

Fig. 2.12 shows the total energy over the whole time interval $(0 ; 1000) \mathrm{s}$. The total energy is calculated for the integration from position $10 \mathrm{~m}$ (close to the influx 

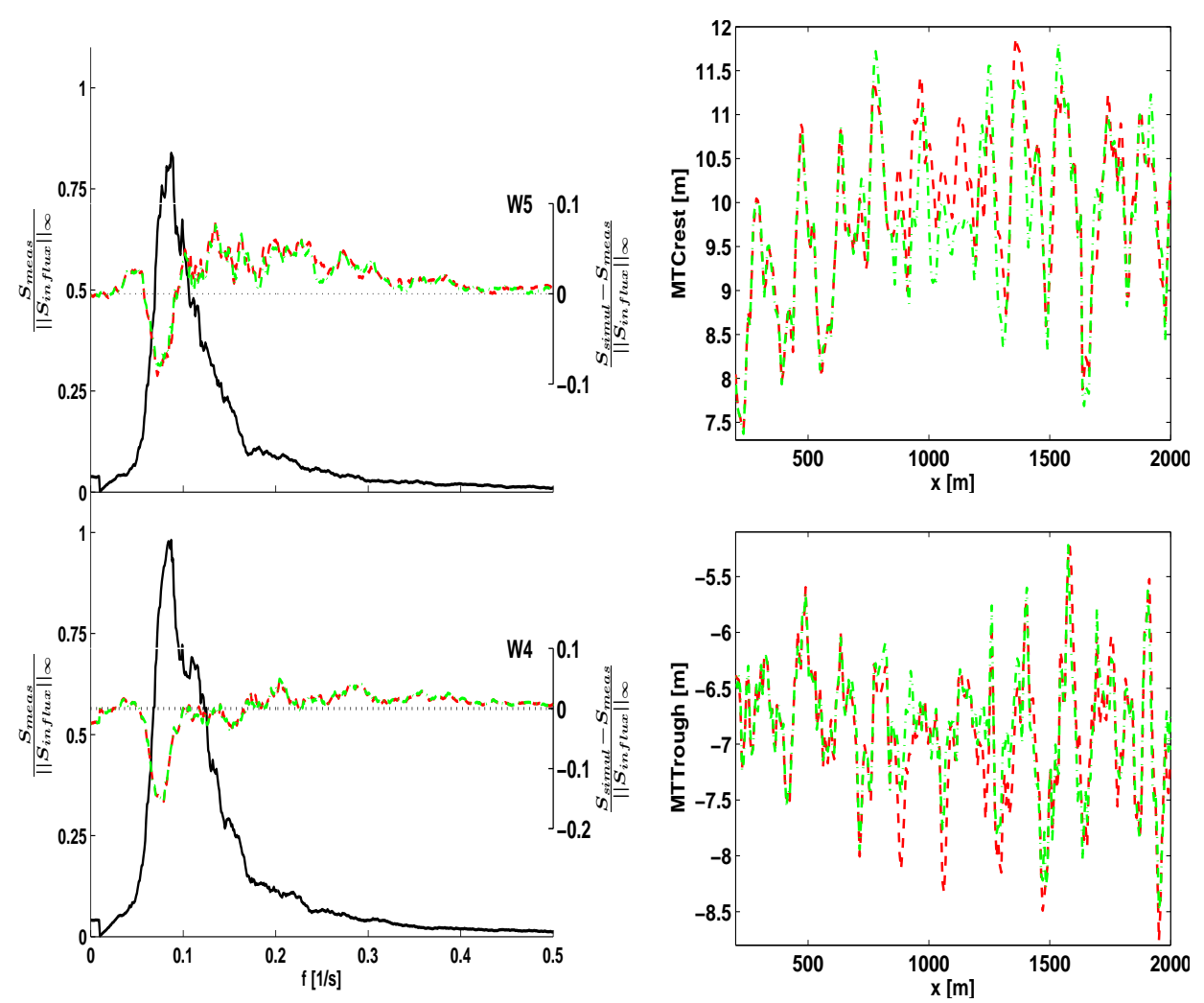

Figure 2.9: Same as Fig. 2.5, now for the breaking models ABHS4break with $B(N)$ (red, dashed), and ABHS4break with $B(u)$ (green, dashed-dot).

Table 2.3: Same as Table 2.1, now for ABHS4break with $B(N)$ and ABHS4break with $B(u)$.

\begin{tabular}{lccccc}
\hline & \multicolumn{4}{c}{ Correlation } & Crel \\
& W2 & W3 & W4 & W5 & \\
\hline ABHS4 & 0.921 & 0.917 & 0.895 & 0.735 & 0.35 \\
ABHS4break $B(N)$ & 0.921 & 0.917 & 0.894 & 0.868 & 0.54 \\
ABHS4break $B(u)$ & 0.921 & 0.917 & 0.895 & 0.873 & 0.57 \\
\hline
\end{tabular}

position) to $3695 \mathrm{~m}$ (end of the domain). The dissipation of energy by the wave breaking model is clearly visible. The difference of energy dissipation for the viscous model $\mathrm{B}(\mathrm{u})$ and $\mathrm{B}(\mathrm{N})$ is rather small. The large variations in the total energy are due to the influxing and damping process. 


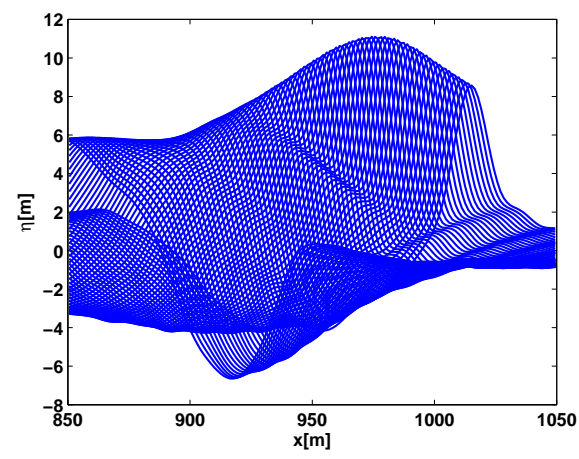

Figure 2.10: Same as Fig. 2.7 now for ABHS4break with B(u). The breaking process starts at position $958 \mathrm{~m}$, time $221.6 \mathrm{~s}$ and the breaking continues for $9.8 \mathrm{~s}$.

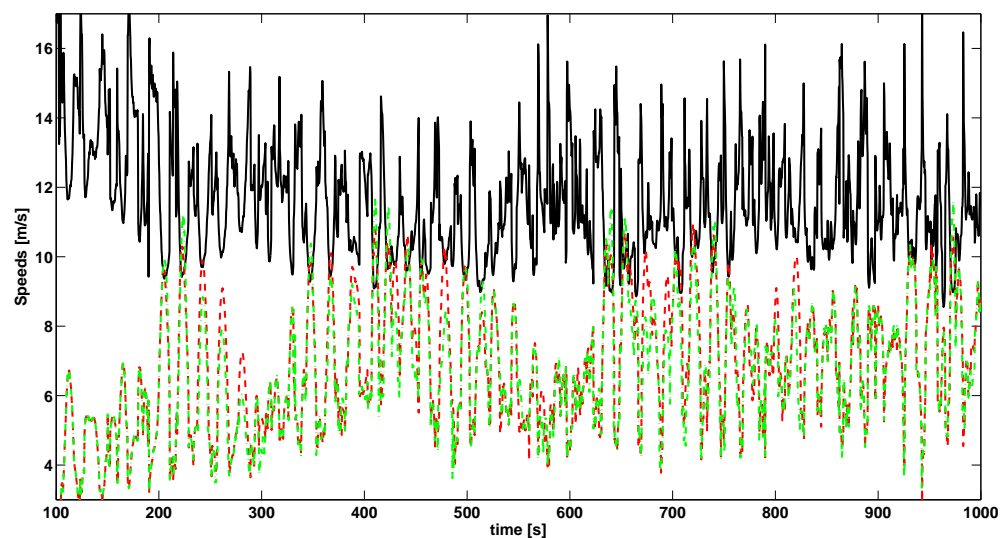

Figure 2.11: Same as Fig. [2.6 now for ABHS4break with $B(N)$ (red, dashed) and ABHS4break with $B(u)$ (green, dashed-dot).

\subsubsection{Wave breaking over a bar Beji and Battjes, 1993]}

Wave propagation over a submerged trapezoidal bar involves a number of complex processes such as the amplification of the bound harmonics during the shoaling process, wave breaking on the top of the bar, and wave decomposition in the deepening part (downslope). Therefore, it is a challenging test case for numerical models to give a good description of these transformations.

Beji and Battjes [1993] conducted a series of experiments concerning the propagation of regular waves over a submerged trapezoidal bar, corresponding to either non-breaking, spilling breaking or plunging breaking waves. In this section, we focus on the long wave plunging case $(\mathrm{f}=0.4 \mathrm{~Hz})$. This case has been successfully simulated for the first time by Tissier et al. 2012].

The bathymetry is presented in Fig. 2.13 the water depth varies from $0.4 \mathrm{~m}$ in the deeper region to $0.1 \mathrm{~m}$ over the top of the bar. In the experiment at eight positions the wave height is measured: s1, s2, s3, s4, s5, s6, s7, s8 at positions 


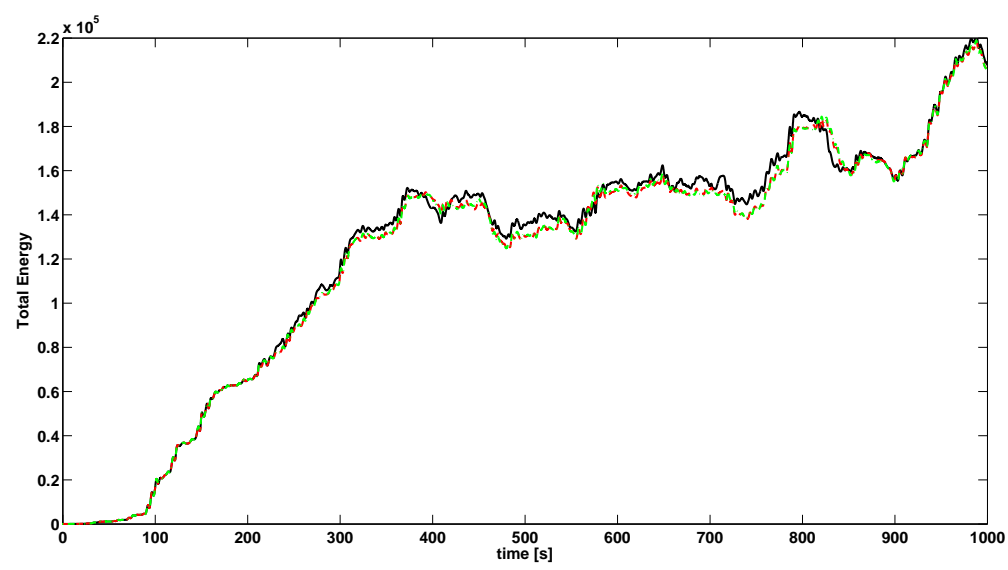

Figure 2.12: Plot of the total energy of ABHS4 (black, solid), ABHS4break with $B(N)$ (red, dashed), ABHS4break with B(u) (green, dashed-dotted).

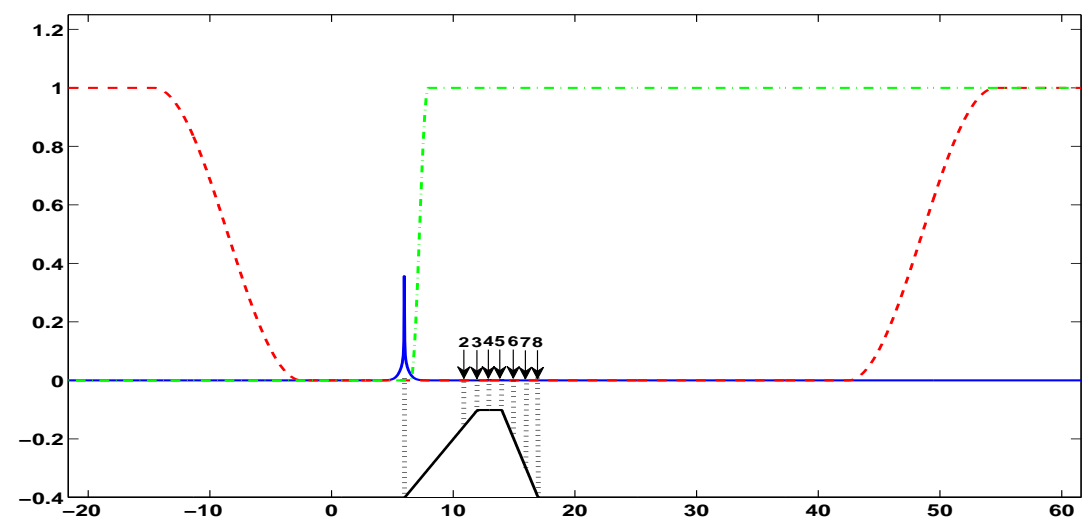

Figure 2.13: The spatial calculation domain for the Beii and Batties [1993 case. The generation function $\gamma(x)$ centered at 11 (blue, solid) is used in the source function. Shown are also the smooth characteristic functions for the damping zone (red, dashed), the nonlinear adaptation function (green,dashed-dot) and the trapezoidal bar (black, solid) with measurement positions (black, arrow).

$x=6,11,12,13,14,15,16,17 \mathrm{~m}$, respectively. Position $\mathrm{s} 2$ is at the upslope area (a 1:20 slope), positions s3, s4, s5 are at the top of the bar and positions s6, s7, s8 are at the downslope area (a 1:10 slope). In the experiment, a beach with a 1:25 slope was present at $18.95 \mathrm{~m}$ to $37.7 \mathrm{~m}$ that is not considered in our numerical simulation. The measured surface elevation at the first position s1 at the foot of the slope is used as influx signal for our simulations.

We now describe the results for the breaking models ABHS2break, and ABHS3break with criteria $U / C>0.7$ and $u^{F}=0.2 u^{I}$. In Fig. 2.14, we show 


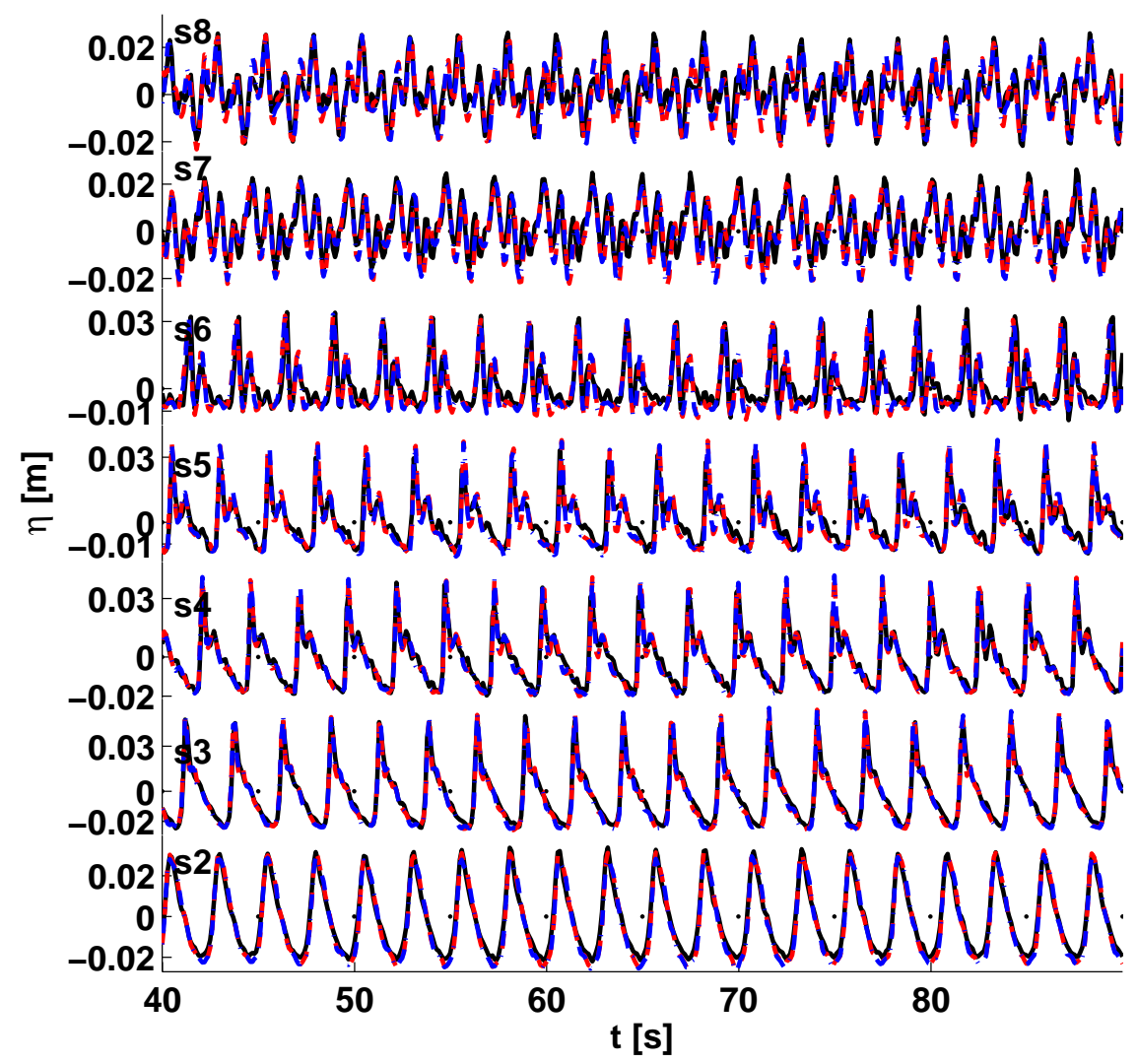

Figure 2.14: Elevation time traces for Beii and Batties [1993] case, in the time interval (40;90) s at positions s2 to s8. Shown are the measurement (black, solid), simulation with ABHS2break (red, dashed) and ABHS3break (blue, dashed-dot).

the results for the elevation time traces at all measurement points in the time interval $(40 ; 90)$ s. The simulated surface elevation is in good agreement with the measurements as shown in Fig. 2.14. The wave shape is well-reproduced and in phase during the shoaling process (at s2), at the onset of breaking (at s3) and during breaking at the top of the bar (at s4 and s5). After the top above downslope, the breaking stops and the wave decomposes in several smaller amplitude waves (at s6, s7 and s8). The simulation shows an underestimation of the crest height at s7 and $\mathrm{s} 8$.

In Fig. 2.15, we show the corresponding normalized amplitude spectra which describe the generation (upslope) and annihilation (downslope) of bound harmonics. The first harmonic $(0.4 \mathrm{~Hz})$ until the fifth harmonic $(2 \mathrm{~Hz})$ are clearly visible with decreasing normalized amplitude of spectrum in the upslope area (at s2). On the top 


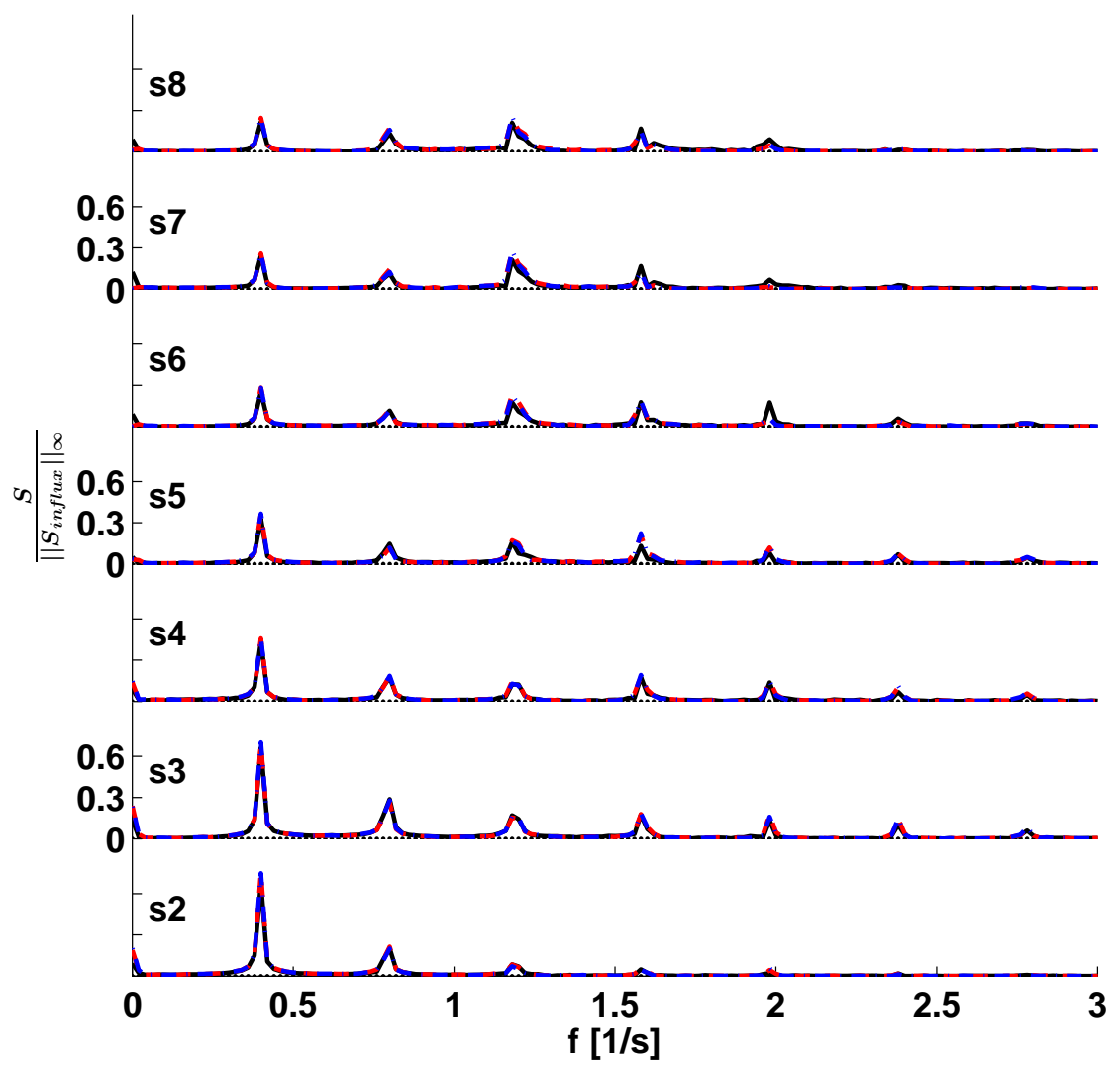

Figure 2.15: Normalized amplitude spectra for Beji and Batties [1993] case; measurement (black, solid) and simulations with ABHS2break (red, dashed), ABHS3break (blue, dasheddot) at position s2 to $s 8$.

(at s3, s4 and s5), the seventh harmonic $(2.8 \mathrm{~Hz})$ is clearly visible which reduces again to fifth harmonic in the downslope area (at s6, s7 and s8). A good agreement with the measurement is also shown by the simulated spectra. However, the simulation shows an underestimation of the amplitude spectrum at the sixth harmonics at s6, s7 and s8.

In Fig. 2.16, we show at the top the spatial evolution of the maximum temporal crest and minimum temporal trough (left) and the mean water level (right). Fig. 2.16 bottom shows the spatial evolution of the significant wave height, skewness $(S k)$ and asymmetry $(A s)$. The asymmetry and skewness are defined as Kennedy et al. 

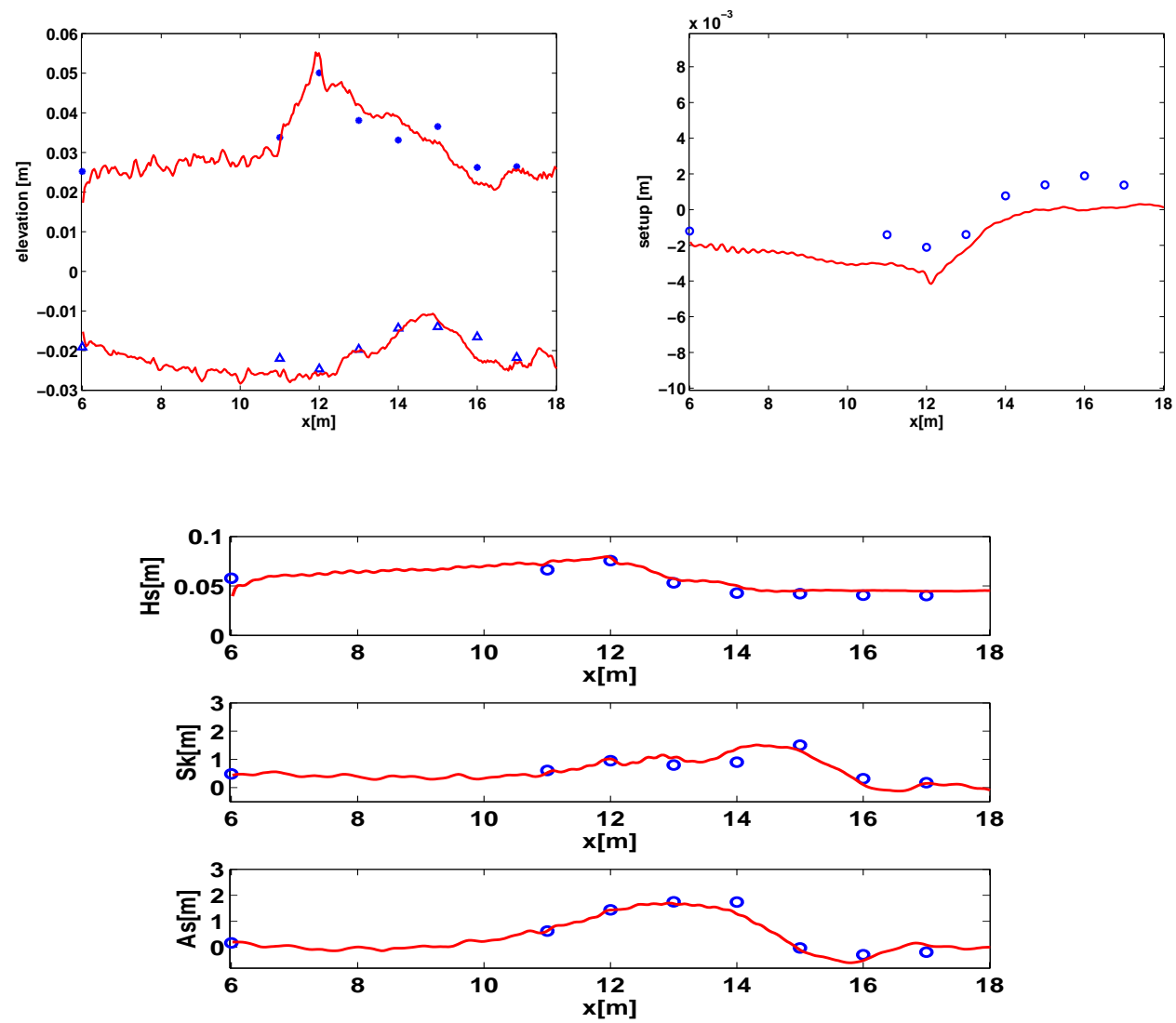

Figure 2.16: Spatial evolution of wave characteristics as computed by ABHS3break (red, lines) and measured (blue, symbols) for Beii and Batties [1993] case. At the left top, *: maximum temporal crest; $\triangle$ : minimum temporal trough, at the right top, o: mean water level. At the bottom, Hs: significant wave height (top), Sk: skewness (middle), As: asymmetry (bottom).

[2000], Tissier et al. 2012]):

$$
\begin{aligned}
& S k=\frac{\left\langle(\eta-\bar{\eta})^{3}\right\rangle}{\left\langle(\eta-\bar{\eta})^{2}\right\rangle^{3 / 2}} \\
& A s=-\frac{\left\langle\mathbb{H}(\eta-\bar{\eta})^{3}\right\rangle}{\left\langle(\eta-\bar{\eta})^{2}\right\rangle^{3 / 2}}
\end{aligned}
$$

where $\bar{\eta}$ is the wave-averaged surface elevation, \langle\rangle is time averaging operator and $\mathbb{H}$ the Hilbert transform. Asymmetry increases steadily as the wave approaches the top of the bar and decreases at the downslope. Skewness increases as the wave shoals and breaks, and then decreases at the downslope. Positive skewness corresponds to narrow crests and flat troughs. The plots show that the simulation captures the 
main wave characteristics in a good way, as confirmed in a different way by Table 2.4 .

Table 2.4: Same as Table 2.1, now for periodic wave breaking over a bar.

\begin{tabular}{lcccccccc}
\hline & \multicolumn{9}{c}{ Correlation } & & Crel \\
& s2 & s3 & s4 & s5 & s6 & s7 & s8 & \\
\hline ABHS2 & 0.989 & 0.977 & 0.841 & 0.702 & 0.589 & 0.491 & 0.54 & 0.46 \\
ABHS2break & 0.99 & 0.983 & 0.961 & 0.912 & 0.838 & 0.832 & 0.851 & 1.15 \\
ABHS3 & 0.991 & 0.984 & 0.83 & 0.719 & 0.664 & 0.589 & 0.617 & 0.84 \\
ABHS3break & 0.986 & 0.988 & 0.964 & 0.918 & 0.863 & 0.867 & 0.884 & 1.52 \\
\hline
\end{tabular}

The quantitative data in Table 2.4 show that the breaking models ABHS2break and ABHS3break has a higher correlation, an improvement with approximately 20 to $30 \%$ compared to the nonbreaking models ABHS2, and ABHS3. The higher order models show better correlation, at the cost of somewhat increased relative computation time (Crel) as expected.

\subsection{Conclusion and remarks}

The derivation of the higher order ABHS equations from the basic Hamiltonian formulation is rather straightforward by using the Taylor expansion around the still water level of the potential and the vertical velocity at the deformed wave profile. The mix of the polynomial expansion in the spatial elevation $\eta$ and the global pseudo-differential operators in the second and higher order terms, works out correctly provided the composition of the operators is retained. This is also true if instead of the non-algebraic exact dispersion relation an approximation is used; if the approximation is algebraic, discretization methods such as finite difference and finite element can be used. In our pseudo-spectral implementation, the exact dispersion properties can be retained, which makes it possible to deal with applications in which waves of very different wave length play a role. This improves the properties of more classical Boussinesq type of equations, and the Hamiltonian consistent approximation guarantees correct evolution of momentum and (approximate) energy.

From the large collection of published breaking models, we have chosen to modify the well known Kennedy et al. [2000] viscous breaking model; the modification can not only deal with long waves but also with fully dispersive equations. This is shown for the test case, an irregular wave with peak wavelength of $211 \mathrm{~m}$ above a depth of $510 \mathrm{~m}$. For this case, a comparison with measurements show that the time traces of simulations at the measurement positions are quite accurate; the substantial changes in measured spectra at the successive positions is reasonably well simulated, although near the peak frequency a somewhat smaller energy can be observed. Two different viscous decay models, one governed by the normal velocity and one by the tangential velocity, give for practical applications almost similar results. In that respect it is interesting to observe that also the non-breaking model ABHS4 performs quite well away from the breaking events; near breaking, with ABHS4 the elevation is calculated too high which also leads to a small shift in the high wave position. 
For the test case of periodic wave plunging breakers over a trapezoidal bar, the comparison with measurements show that the time traces of simulations at the measurement positions are quite accurate; the wave shape is well reproduced during shoaling, breaking and the wave decomposition process. The spectra are also well predicted, showing accurately the bound harmonic generation and annihilation. 


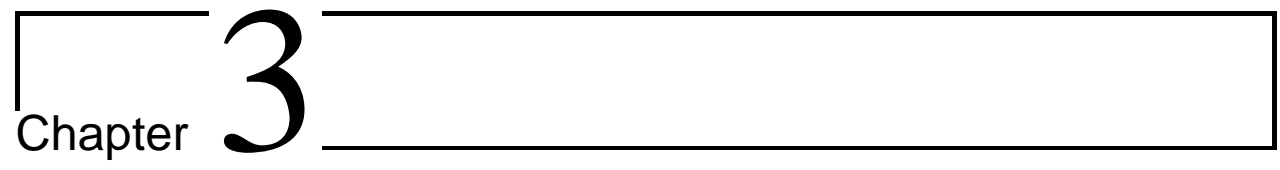

\section{Localization for}

\section{spatial-spectral}

\section{implementations of 1D \\ Analytic Boussinesq equations 1}

\section{Abstract}

Accurate simulations of waves in oceanic and coastal areas should take dispersive effects over a large range of frequencies into account in the relevant order of nonlinearity of the equation. Taking the exact Hamiltonian-Boussinesq formulation of surface waves as starting point, a fully Hamiltonian consistent spatial-spectral approximation of the kinetic energy was shown Kurnia and van Groesen, 2014a to lead to a phase velocity operator that depends on the varying bottom and second, third and fourth order accurate on the surface elevation. In this paper we describe and show with simulations of various $1 \mathrm{D}$ cases of breaking and non-breaking waves how localized effects of partially or fully reflective walls, run-up on a coast and the dam-break problem can be dealt with in the implementation with global Fourier integral operators; a dynamic or post-processing step will show to recover the interior flow properties.

\footnotetext{
${ }^{1}$ Submitted for publication as:

R. Kurnia and E. van Groesen. Localization for spatial-spectral implementations of 1D Analytic Boussinesq equations.
} 


\subsection{Introduction}

This paper starts with a concise summary of a spatial-spectral model for water waves derived recently Kurnia and van Groesen, 2014a and extends the possibilities of this model to deal in particular with localized structures such as walls. The model has a relatively simple implementation using nonlocal Fourier Integral Operators that can time-accurately and efficiently simulate a large variety of waves in complicated geometries that are encountered in coastal engineering applications.

Applications typically deal with a combination of irregular wind waves and swell, that reach the shallower coastal area, run up on the coast or enter and reflect from structures and harbours. For the design of fixed or flexible structures in the coastal area such as breakwater, wind mills, oil platforms, ships, etc. knowledge of expected forces in extreme waves are of paramount importance. Experiments in wave tanks are often used to obtain (impact) data in well-controlled circumstances. The experiments require to prepare the incoming wave such that the desired extreme behaviour takes place at the defined position of the structure. Accurate and efficient simulations can then be helpful in such applications to optimize the experiments.

Simulations of surface waves based on Boussinesq models, that avoid the interior calculation of the flow in full detail, are much more efficient for such applications than full CFD-codes or potential codes. For simulations of high quality, it is necessary that the dispersion is modelled correctly over a large range of wave lengths. Since the dispersion relation of linear waves is given by a non-algebraic function, models with local differential operators for implementations with finite differences or finite elements, require that the dispersion is approximated by an algebraic relation, which leads to restrictions on the range of wave lengths that are modelled correctly. Airy's theory that is exact for linear waves propagating above a flat bottom show that Fourier methods, using pseudo-differential operators (PDO), are required to avoid that restriction. In previous papers Kurnia and van Groesen, 2014a, van Groesen and Andonowati, 2011, van Groesen and van der Kroon, 2012. it was shown how Airy's theory can be extended to include bottom variations using Fourier integral operators (FIO) to replace the PDO's.

Although technically a bit more complicated, using an efficient approximation for the calculation with FIO's, models were derived with straightforward implementations that lead to fast simulations with excellent dispersive properties in second, third and fourth order of nonlinearity. Even wave breaking was included in the model.

The strategy is to use Hamiltonian consistent modelling starting with the fact that the irrotational motion in a layer of inviscid, incompressible fluid can be described as a dynamical system with a Hamiltonian structure and with the surface elevation and the fluid potential at the surface as canonical variables Luke, 1967, Zakharov, 1968, Broer, 1974, Miles, 1977]. Then, in order to obtain explicit equations, the kinetic energy part of the Hamiltonian has to be expressed in the canonical variables, which requires an approximation of the interior potential flow. Such Hamiltonian consistent approximations have exact conservation of the approximated energy.

Generalizing the pseudo-differential operator associated with the linear 
phase speed operator of Airy's theory, a model above varying bottom that is second, third and fourth order accurate in the wave height was derived van Groesen and Andonowati, 2011, van Groesen and van der Kroon, 2012, Kurnia and van Groesen, 2014a]. The coupled spatial-spectral model that results keeps the full profit of dispersive quality; for instance oceanic waves can be dealt with accurately. In this paper we show that a second order accurate model is obtained if the nonlinear phase speed operator is used, simplifying somewhat the second-order expression in Kurnia and van Groesen, 2014a].

However, Fourier expansion techniques, different from finite difference or finite element methods, lead to some problems when complicated geometric structures need to be included. Since we could not find references in the literature, this paper is mainly concerned with the inclusion of such fixed structures in a spatial-spectral setting. (In a forthcoming paper we will deal with floating and moving ships in interaction with ships and structures.) In particular, we will describe and illustrate a localization method how to deal with fully or partially reflecting walls, and even with prescribed frequency dependent reflections at a wall. Another example deals with the run-up of waves on a coast by taking at each instant the shore line as the position where the total depth vanishes at time; this method is valid without restrictions on the shape of the coastal bathymetry.

The fact that Boussinesq models do not explicitly calculate the interior flow, which may be needed for various applications, this does not mean it cannot be calculated, in a time dynamic way (at each time step), or in a post-processing step. The way how to calculate the flow and pressure, will be described, and for the nontrivial case of breaking waves during run-up, the quality will be illustrated by comparison with measurements.

The outline of the paper is as follows. Section 3.2 describes the spatial-spectral modelling in the Hamiltonian structure and the calculation of interior flow properties. Section 3.3 deals with localization for partially or fully reflecting walls, frequency dependent reflecting walls and run-up on a coast. Results of various test cases are presented in Section 3.4. Simulations are validated with experimental data or theoretical exact results. Conclusions and remarks will finish the paper.

The models and methods presented in this paper are part of HAWASSI software (Hamiltonian Wave Ship Structures Interactions) that has been developed over the past years.

\subsection{Spatial-spectral modelling within the Hamilto- nian structure}

In this section we start with the description of the Hamiltonian structure. We show by way of illustration the consistent approximation of the Kinetic energy in the limiting cases of Shallow Water and Airy's linear wave theory. Then we show a second order accurate approximation above bathymetry. Dissipation models for wave breaking and bottom friction are briefly explained. In the last subsection we describe the procedure to calculate the internal flow and pressure. 


\subsubsection{Hamiltonian structure}

Waves on a layer of incompressible, inviscid fluid can be described for irrotational internal fluid motion by canonical variables the elevation $\eta(x, t)$ and the fluid potential $\phi(x, t)$ that depend on the spatial variable $(x)$ and time $(t)$. This was described by Zakharov 1968], Broer [1974], and follows from Luke's variational action principle [Luke, 1967] as was shown by Miles [1977]. The action principle for Hamiltonian $\overline{\mathcal{H}}$ is given by

$$
\int\left[\int \phi \partial_{t} \eta d x-\overline{\mathcal{H}}(\phi, \eta)\right] d t
$$

for which the critical points, just as in Classical Mechanics, satisfy the Hamilton equations:

$$
\begin{aligned}
& \partial_{t} \eta=\delta_{\phi} \overline{\mathcal{H}}(\phi, \eta) \\
& \partial_{t} \phi=-\delta_{\eta} \overline{\mathcal{H}}(\phi, \eta) .
\end{aligned}
$$

For notational convenience in the following we also introduce the tangential velocity $u(x, t)=\partial_{x} \phi(x, t)$. Using this variable, the Hamiltonian system transforms with $\mathcal{H}(u, \eta)=\overline{\mathcal{H}}(\phi, \eta)$ to

$$
\begin{aligned}
& \partial_{t} \eta=-\partial_{x} \delta_{u} \mathcal{H}(u, \eta) \\
& \partial_{t} u=-\partial_{x} \delta_{\eta} \mathcal{H}(u, \eta) .
\end{aligned}
$$

The Hamiltonian is the sum of potential and kinetic energy

$$
\begin{aligned}
\overline{\mathcal{H}}(\phi, \eta) & =P(\eta)+K(\phi, \eta) \\
P(\eta) & =g \iint_{-D}^{\eta} z d z d x=\frac{g}{2} \int\left(\eta^{2}-D^{2}\right) d x \\
K(\phi, \eta) & =\frac{1}{2} \iint_{-D}^{\eta}|\nabla \Phi|^{2} d z d x .
\end{aligned}
$$

In the expression for $K$ it is understood that $\Phi$ satisfies the Laplace equation in the interior with vanishing normal derivative at the impermeable bottom and at lateral boundaries. This can be succinctly described with Dirichlet's principle Riemann, 1857] as

$$
K(\phi, \eta)=\min _{\Phi}\left\{\frac{1}{2} \iint_{-D}^{\eta}|\nabla \Phi|^{2} d z d x \mid \Phi(x, \eta)=\phi\right\} .
$$

With the normal velocity at the free surface $\partial_{N} \Phi=\nabla \Phi(x, \eta) \cdot\left(-\partial_{x} \eta, 1\right)$, the kinetic energy can be written with Green's identity as

$$
K(\phi, \eta)=\left.\frac{1}{2} \int \phi \partial_{N} \Phi\right|_{z=\eta} d x .
$$

The variational derivative with respect to $\phi$ is given by

$$
\delta_{\phi} K=\partial_{N} \Phi
$$


and shows that $\delta_{\phi} K$ is the Dirichlet to Neumann (DtN) operator, mapping the Dirichlet value $\phi(x)$ to the Neumann expression $\partial_{N} \Phi$.

In order to express $K$ in the basic variables $\phi, \eta$ explicitly, approximations of $K$ based on Dirichlet's principle will be made; then the approximated $K$ is used to calculate the approximate $D t N$ operator. In the following two subsections we show approximations for limiting cases and second order accurate approximations.

\subsubsection{Limiting cases}

In this section we show the approximation of $K$ for limiting cases such as Shallow water and Airy's linear wave theory.

\section{Shallow Water}

The simplest approximation is to restrict the minimization in (Eq. 3.5) to the single function $\Phi(x, z, t)=\phi(x, t)$. Then

$$
K_{\mathrm{SW}}(\phi, \eta)=\frac{1}{2} \int H(x, t)\left(\partial_{x} \phi\right)^{2} d x
$$

in which the total depth $H(x, t)=D(x)+\eta(x, t)$. Observe that $K$ can be written with the nonlinear phase velocity $C=\sqrt{g H}$ as $K_{\mathrm{SW}}(u, \eta)=\frac{1}{2 g} \int(C u)^{2} d x$. This leads correctly to the shallow water equations when we write down the Hamilton equations for the Hamiltonian $\frac{1}{2} \int g \eta^{2} d x+K_{\mathrm{SW}}(u, \eta)$. The other parts of this section can then be summarised as the method to generalise this for waves with arbitrary wavelength.

\section{Airy's linear wave theory}

Starting with a flat bottom at depth $D_{0}$, the linear theory is obtained using Fourier transformation. For convenience we introduce a compact notation to denote the direct and inverse Fourier transformation by $f(x) \hat{=} \hat{f}(k)$ and $\hat{f}(k) \doteq f(x)$ as short hand for

$$
\hat{f}(k)=\int f(x) e^{-i k x} d x \quad \text { and } \quad f(x)=\frac{1}{2 \pi} \int \hat{f}(k) e^{i k x} d k .
$$

Then the solution of the Laplace problem above flat bottom with depth $D_{0}$

$$
\Phi(x, z) \hat{=} \hat{\phi}(k) \frac{\cosh k\left(z+D_{0}\right)}{\cosh k D_{0}}
$$

leads to the explicit expression of the kinetic energy as

$$
K_{0}(u)=\frac{1}{2 g} \int\left(C_{0} u\right)^{2} d x
$$

where $C_{0}$ is the pseudo-differential operator (PDO) given by $C_{0} u \hat{=} \hat{C}_{0}\left(k, D_{0}\right) \hat{u}(k)$. The symbol of $C_{0}$ is the phase velocity $\hat{C}_{0}\left(k, D_{0}\right)=\sqrt{g \tanh \left(k D_{0}\right) / k}$. Note that since $\hat{C}_{0}$ is a real, even function of $k$, the operator $C_{0}$ is real and symmetric in $L_{2}$-inner product. 
The Hamilton equations for linear waves above flat bottom are given by

$$
\begin{aligned}
& \partial_{t} \eta=-\frac{1}{g} \partial_{x}\left(C_{0}^{2} u\right) \\
& \partial_{t} u=-\partial_{x}(g \eta)
\end{aligned}
$$

which leads to the well known dispersion relation for time harmonic solutions

$$
\omega^{2}=k^{2} \hat{C}_{0}^{2}=g k \tanh k D_{0} .
$$

\subsubsection{Second order accurate approximation above bathymetry}

To include nonlinearity in the model, we take the total depth $H(x, t)=D(x)+\eta(x, t)$ in the expression for the nonlinear phase operator. With the kinetic energy as

$$
K=\frac{1}{2 g} \int(C(\eta) u)^{2} d x
$$

the action of $C(\eta)$ is given by

$$
C(\eta) u \hat{=} \hat{C}(k, H(x, t)) \hat{u}(k) .
$$

where the symbol of the nonlinear phase velocity is given by

$$
\hat{C}(k, H(x, t))=\sqrt{g \tanh (k H(x, t)) / k} .
$$

Note that this is the correct expressions for the two limiting cases SWE and linear waves. The action of $C(\eta)$ can also be written in full as an Fourier integral operator (FIO) with kernel $\tilde{C}(x, y)$

$$
C(\eta) u(x)=\int \tilde{C}(x, y) u(y) d y=\frac{1}{2 \pi} \iint \hat{C}(k, H(x)) u(y) e^{i k(x-y)} d k d y .
$$

This operator is not symmetric; the adjoint $C^{*}(\eta)$ has kernel $\tilde{C}(y, x)$ that is given by

$$
C^{*}(\eta) u(x)=\int \tilde{C}(y, x) u(y) d y=\frac{1}{2 \pi} \iint \hat{C}(k, H(y)) u(y) e^{-i k(x-y)} d k d y .
$$

The normal derivative at the surface is then given by the result of the variational derivatives

$$
\delta_{u} K=\frac{1}{g} C(\eta)^{*} C(\eta) u
$$

in which $C(\eta)^{*} C(\eta)$ is a symmetric operator. The variational derivative with respect to $\eta$ is somewhat more complicated:

$$
\delta_{\eta} K=\frac{1}{g} C(\eta) u .\left(\partial_{\eta} C\right) u
$$


where the operator $\left(\partial_{\eta} C\right) u \hat{=} \frac{g}{2} \hat{C}^{-1}(k, H(x, t))\left(1-\tanh ^{2}(k H(x, t))\right) \hat{u}(k)$.

Observe that since $\eta$ itself determines 'short' spatial variations, this nonlinear phase velocity operator is not slowly varying, so this is not a quasi-homogeneous approximation. Nevertheless, it easily seen that in the limit for long waves, $C(\eta)$ approaches the correct nonlinear expression of SWE. But more importantly, with this expression for $C(\eta)$ we get a wave model that is accurate in second order. This will now be shown.

Expanding the Fourier integral operator up to second order in $\eta$ gives

$$
C(\eta) u=C_{0} u+\eta C_{1} u .
$$

where $C_{0}, C_{1}$ are pseudo-differential operators for a constant depth $D$ and expressed by

$$
C_{0} u \hat{=} \hat{C}(k, D) \hat{u}(k) ; \quad C_{1} u \hat{=} \hat{C}^{\prime}(k, D) \hat{u}(k) .
$$

The first derivative of the symbol $\hat{C}_{1}$ with respect to $\eta$ is

$$
\hat{C}^{\prime}(k, D)=\frac{g}{2} \hat{C}_{0}^{-1}\left(1-\tanh ^{2}(k D)\right) .
$$

Substituted in the kinetic energy Eq. 3.10, the result can be written up to third order with the terms of the same order grouped together in square brackets as

$$
K=\frac{1}{2 g} \int\left[C_{0} u\right]^{2}+\left[2 C_{0} u . \eta C_{1} u\right] d x .
$$

The second and third order terms in Eq. 3.13 are the same as the second and third kinetic energy terms in Kurnia and van Groesen, 2014a. However, higher order terms turn out to be different than in Kurnia and van Groesen, 2014a]. Hence, it must be concluded that the expansion via the phase velocity agrees in second order and third order of the kinetic energy.

A remark about the numerical implementation of pseudo-differential operators (PDO) and Fourier integral operators (FIO) concerns the efficiency. A PDO can be handled efficiently using a single FFT; for a Fourier operator, a FFT is required for each spatial grid point, which is very expensive in total. However, the computation time is substantially reduced (to a few FFT's) by using a piecewise constant approximation, or by an interpolation method; see Kurnia and van Groesen, 2014a, van Groesen and van der Kroon, 2012] for more details.

\subsubsection{Wave breaking and bottom friction}

Dissipation models due to wave breaking $\left(R_{b}\right)$ and bottom friction $\left(R_{f}\right)$ can be applied by adding specific terms in the momentum equation as

$$
\partial_{t} u=\cdots+R_{b}+R_{f}
$$

The breaking process includes energy dissipation, an initiation and an termination mechanism. The method described in detail in Kurnia and van Groesen, 2014a 
takes an energy dissipation mechanism based on eddy viscosity, and the initiation and termination mechanism are based on a kinematic criterion. The method is a generalization of [Zelt, 1991, Kennedy et al., 2000] for dispersive equations. 2001.

The bottom friction term $\left(R_{f}\right)$ is given by a Chezy expression Bühler and Jacobson,

$$
R_{f}=-\frac{C_{f}}{D+\eta} u|u|
$$

where $C_{f}$ is the bottom friction coefficient, typically in the range of $\left(10^{-3} ; 10^{-2}\right)$, depending on the Reynolds number and bottom condition [Lynett et al., 2002].

\subsubsection{Internal flow and pressure}

In Boussinesq type of models, the internal flow that should satisfy the Laplace equation is not calculated but approximated so that the dynamics can be formulated in the surface variables only. This section shows how the internal flow can be calculated directly in the time dynamics (at each time step) or can be recovered in a post-processing step.

Given the explicit expression of the fluid potential $\Phi(x, z, t)$ that is found from a nonlinear extension of the Airy potential as

$$
\Phi(x, z, t)=\int \hat{\phi}(k) \frac{\cosh k(z+D(x))}{\cosh k(\eta(x, t)+D(x))} e^{i k x} d k,
$$

the velocity and acceleration components can be calculated in time and at horizontal and vertical spatial positions. The horizontal and vertical velocity which are expressed by $\partial_{x} \Phi$ and $\partial_{z} \Phi$, respectively, are given by the expressions below, in which we use a recurrent expression denoted by $\Gamma$

$$
\begin{aligned}
\Gamma= & \int \hat{\phi}(k, t) \frac{\cosh k(z+D(x))}{\cosh k(\eta(x, t)+D(x))}[-k \tanh k(\eta(x, t)+D(x))] e^{i k x} d k \\
\partial_{x} \Phi= & \int i k \hat{\phi}(k, t) \frac{\cosh k(z+D(x))}{\cosh k(\eta(x, t)+D(x))} e^{i k x} d k \\
& \quad+\partial_{x}(\eta(x, t)+D(x)) \cdot \Gamma+\partial_{x} D \cdot \partial_{z} \Phi \\
\partial_{z} \Phi= & \int \hat{\phi}(k, t) \frac{k \sinh k(z+D(x))}{\cosh k(\eta(x, t)+D(x))} e^{i k x} d k .
\end{aligned}
$$

The accelerations can be calculated either analytically or numerically by differentiating the velocities in time.

The dynamic pressure $-\partial_{t} \Phi$, is given by

$$
\partial_{t} \Phi=\int \partial_{t} \hat{\phi}(k, t) \frac{\cosh k(z+D(x))}{\cosh k(\eta(x, t)+D(x))} e^{i k x} d k+\partial_{t} \eta \cdot \Gamma
$$

with the aid of which the total pressure

$$
\mathcal{P}=-\partial_{t} \Phi-g z-\frac{1}{2}\left(\left(\partial_{x} \Phi\right)^{2}+\left(\partial_{z} \Phi\right)^{2}\right) .
$$


can easily be calculated.

Observe that the dynamic pressure requires the time derivative of surface variables $\left(\partial_{t} \eta\right.$ and $\left.\partial_{t} \phi\right)$. These terms can be obtained without additional computations and without loss of accuracy if the results of calculating the right hand sides of the dynamic equations are being stored to be called in the next time step.

Data of interior flow can for instance be used as initial condition in CFD codes that take viscous effects into account for a detailed modelling of the wave-structure interactions, as has been shown for instance in Bunnik and van Groesen, 2008].

\subsection{Localization}

Different from local discretization methods like finite differences or finite elements, special care has to be taken for local structures when dealing with the Fourier Integral operators as in this paper. Sudden changes, as for instance happens with flow reversal because of the collision at a wall, have to be modelled very explicitly in the governing equation instead of by using boundary conditions. In the first subsection we show how a partially or fully reflecting wall can be modelled with an abrupt density (porosity) change. A uniform value behind the wall will reflect all incoming waves with the same factor independent of the wave length. The peculiar advantage of the explicit appearance of the phase velocity in our Fourier modelling will makes it very easy to model a wall where the reflection depends on the incoming wave by making the density dependent on the wave length in a desired prescribed way; this would be much more difficult to achieve in local discretization schemes. In the third subsection it is shown that a moving shore line can be modelled as a moving fully reflecting wall.

\subsubsection{Partially reflecting wall}

Linear wave theory describes the reflection coefficient $R$ of waves running in a medium with phase speed $C_{-}$that abruptly changes into phase speed $C_{+}$by the formula

$$
R=\frac{C_{-}-C_{+}}{C_{-}+C_{+}}
$$

To model a wall at position $x=0$, say, we invoke such reflection by changing the density in the kinetic energy with a quantity 1 before the wall $(x<0)$ to a quantity $\rho^{2}<1$ for $x>0$. The corresponding kinetic energy is then

$$
\frac{1}{2 g} \int^{0}(C u)^{2} d x+\frac{\rho^{2}}{2 g} \int_{0}(C u)^{2} d x .
$$

This corresponds to a change of phase speed with a factor $\rho$ and the linear reflection relation is given by

$$
R=\frac{1-\rho}{1+\rho}
$$

The calculated reflection from simulation results in the next section will confirm this relation although nonlinear waves show a small deviation from the relation for linear waves. 
Conversely, if a desired reflection coefficient $R$ is given, the required value of $\rho$ is found from inversion of the explicit relation:

$$
\rho=\frac{1-R}{1+R}
$$

\subsubsection{Frequency dependent reflecting wall}

The idea of the previous subsection can now be generalized for frequency or wave length dependent reflection, by multiplying the phase velocity with a quantity $\rho(k)$ resulting in the kinetic energy after the wall as

$$
K(u, \eta)=\frac{1}{2 g} \int_{0}\left|C_{\rho} u\right|^{2} d x \text { with } \hat{C}_{\rho}=\rho(k) \hat{C} .
$$

By prescribing the reflection coefficient depending on frequency $\omega, 0 \leq R(\omega) \leq 1$, the quantity $\bar{\rho}(\omega)$ is given by

$$
\bar{\rho}(\omega)=\frac{1-R(\omega)}{1+R(\omega)}
$$

Then the corresponding quantity $\rho$ depending on the wavenumber $k$ can be obtained by

$$
\rho(k)=\bar{\rho}\left(\Omega_{-}^{-1}(\omega)\right)
$$

with $\Omega_{-}$the dispersion relation at the left, $\Omega_{-}(k)=k C_{-}(k)$.

In the next section we illustrate this for the case that the longest waves are completely reflected while other wave lengths are reflected only partially; this may serve as a simple model for the complete refection of infragravity waves and partial reflection of shorter waves when coastal waves bounce against a break water.

\subsubsection{Run-up on coast}

In the modelling of the run-up of waves on a coast, a spatial truncation is applied in the total energy. Then the Hamiltonian is taken to be

$$
\mathcal{H}(\eta, u)=\frac{1}{2} \int\left[g \eta^{2}+\frac{1}{g}(C u)^{2}\right] \chi d x .
$$

and leads to the nonlinear Hamilton equations

$$
\begin{aligned}
& \partial_{t} \eta=-\frac{1}{g} \partial_{x}\left(C^{*}(C u \cdot \chi)\right) \\
& \partial_{t} u=-\partial_{x}\left(g \eta \chi+\frac{1}{g} C u \cdot\left(\partial_{\eta} C\right) u \cdot \chi\right) .
\end{aligned}
$$

The simulation interval is changing due to the moving shoreline. The governing dynamic equations (Eq. 3.25) hold on the wet side or active domain of the changing 
simulation interval. It turns out that a moving Heaviside function $\chi(H)$ can be used to define the wet and dry domain as defined by

$$
\chi(H)=\left\{\begin{array}{ll}
0 & \text { if } H-H_{\min }<0 \\
1 & \text { if } H-H_{\min } \geq 0
\end{array} .\right.
$$

Taking $\chi(H)$ with $H(x, t)=\eta(x, t)+D(x), H_{\text {min }}$ is the minimum total depth that can be simulated depending on the maximal wave number used in the simulation. The minimum total depth is taken to be $H_{\min }=\left(\nu / k_{\text {cut }}\right)^{2} / g$ where $\nu$ the peak frequency and $k_{\text {cut }}=\max (k) / 4$. The $k_{\text {cut }}$ is motivated in the Fourier method to prevent aliasing as described in Kurnia and van Groesen, 2014a, van Groesen and van der Kroon, 2012].

\subsection{Simulation results}

In this section we show the performance of the accurate dispersive models with spatial-spectral implementation in the following inhomogeneous problems: waves over an underwater slope, waves breaking over a bar, breaking waves running up the coast, wave reflection against walls and the dam-break problem.

The simulations results of waves over an underwater slope, wave breaking over a bar and the dam-break problem are obtained by the second order model ABHS2 as presented in Kurnia and van Groesen, 2014a. The simulation results of breaking waves running up the coast and wave reflection against walls are obtained by the second order equation as presented in this paper. The third and fourth order models ABHS3,4 from Kurnia and van Groesen, 2014a] give comparable, slightly better, results, at the cost of somewhat larger computation times.

Area influxing is used as described in Lie et al., 2014], and to avoid periodic looping damping zones are employed at both ends of the interval.

\subsubsection{Irregular waves running up a slope}

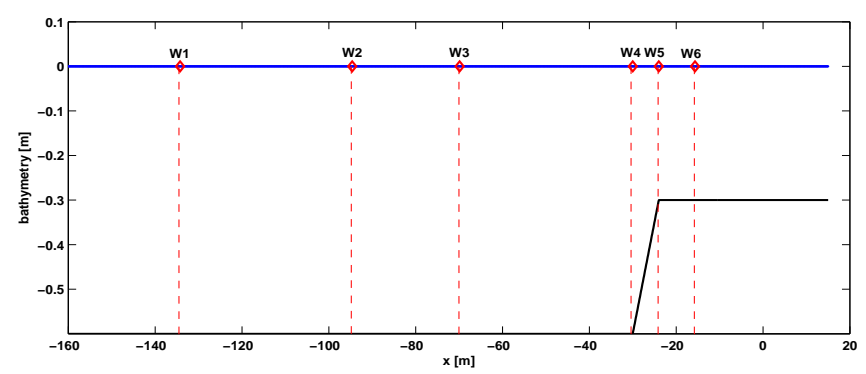

Figure 3.1: Lay out of the experiment of MARINbench 103001 with the location of wave gauges indicated. 


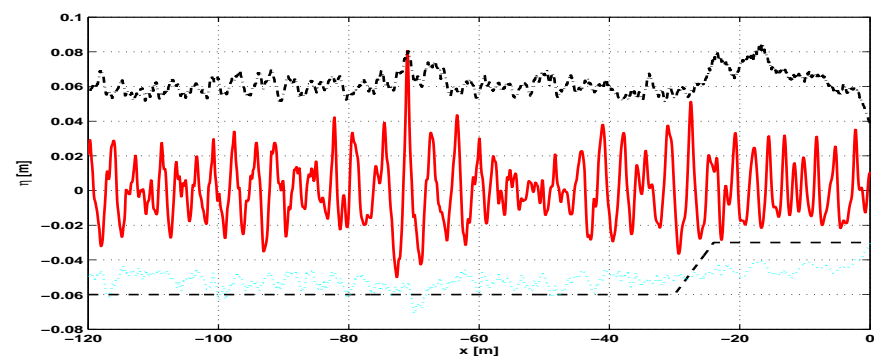

Figure 3.2: Shown are the spatial wave profiles at $(t \approx 282.5 \mathrm{~s})$ at which an extreme crest height is obtained (red, solid-line), the maximum temporal crest (black, dashed-dot) and the minimum temporal trough (cyan, dots) after $700 \mathrm{~s}$; the bathymetry is shown in a scale of (1:10) (dashed-line, black).

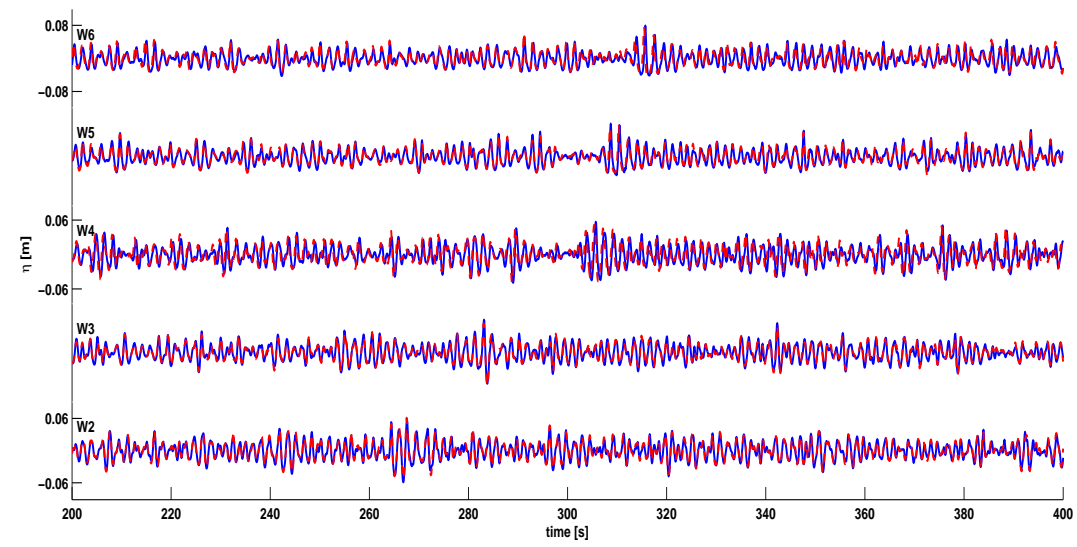

Figure 3.3: Elevation time traces at positions $W 2, x=-94.6 \mathrm{~m}, \mathrm{~W} 3, x=-69.9 \mathrm{~m}, \mathrm{~W} 4, x=-$ $30 \mathrm{~m}, \mathrm{~W} 5, x=-24 \mathrm{~m}$, and $W 6, x=-15.7 \mathrm{~m}$ are shown for the measurement (blue, solid-line) and the simulation (red, dashed-line) of MARINbench 103001.

The test case is a wave that after generation travels above a deep area at depth $0.6 \mathrm{~m}$, then runs-up on an uniform slope (1:20) and continues above a flat shallow part with depth $0.3 \mathrm{~m}$. This is a simplified geometry in laboratory scale (spatial factor 50) for the run-up of waves from the deeper sea to the shallower coast.

The wave is an irregular wave with spectrum of JONSWAP-type and random phases, with peak period $\left(T_{p}\right) 1.7 \mathrm{~s}$ and significant wave height $\left(H_{s}\right) 0.062 \mathrm{~m}$. This wave corresponds to wind waves entering the coastal area with peak period $12 \mathrm{~s}$ and significant wave height $3.1 \mathrm{~m}$. The experiment was performed in MARIN (Maritime Research Institute in Netherlands) and registered as MARINbench 103001. Fig. 3.1 shows the lay out of the experiment and location of measurements. For the simulation, the measurement data at $\mathrm{W} 1$ is used as influx signal.

Simulation results for this case have been presented before in 
van Groesen and van der Kroon, 2012, Adytia and van Groesen, 2012 using BiAB and OVBM equations. Fig. 3.2 shows the spatial wave profile at which an extreme crest is obtained in the approximately 400 wave long wave train. An extreme or freak like wave is usually defined as a wave with wave height more than $2 H_{s}$. The freak-like wave is observed at $\mathrm{t} \approx 282.5 \mathrm{~s}$ and at a position close to measurement at W3. Fig. 3.3 shows the time traces of simulations and measurement. The wave shapes are well reproduced during propagation above the deep area (W2, W3), during run-up from the foot of the slope (W4) to the top (W5) and above the flat shallow part (W6).

The correlation between simulation and measurement gives quantitative information about the accuracy of the simulation. Deviations from the maximal value 1 of the correlation measures especially the error in phase, a time shift of the simulation; for correlation -1 the simulation is in counter phase with the measurement. In the present simulation, the correlation has been calculated in the time interval $(100 ; 700) \mathrm{s}$ at $\mathrm{W} 2, \mathrm{~W} 3, \ldots, \mathrm{W} 6$ to be $0.95,0.94,0.66,0.90,0.81$. The correlation at W4 gives the lowest value as was also obtained in van Groesen and van der Kroon, 2012, Adytia and van Groesen, 2012.

\subsubsection{Irregular wave breaking over a bar}

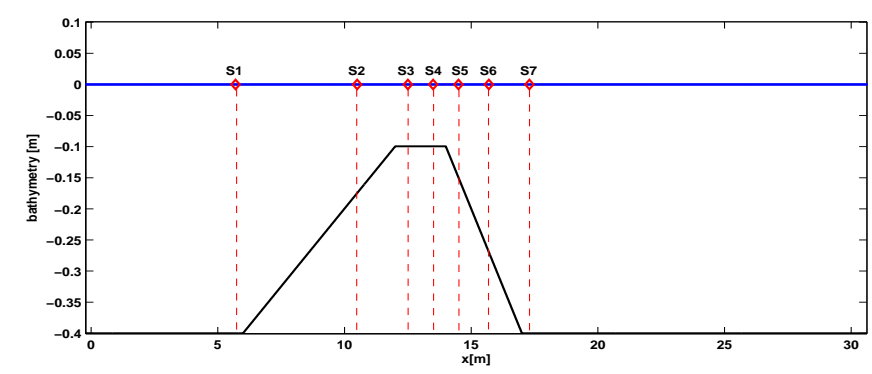

Figure 3.4: Lay out of the experiments of [Beii and Batties, 1994], with the location of the wave gauges.

Beji and Batties [1994, 1993] conducted a series of experiments concerning the propagation of regular and irregular waves over a submerged trapezoidal bar, corresponding to either non-breaking, spilling breaking or plunging breaking waves. In this section we show simulation of an irregular spilling breaking wave, with peak period $1.7 \mathrm{~s}$ and significant wave height $0.035 \mathrm{~m}$. Simulations using ABHS2,3 equations for regular wave plunging breaking have been shown in Kurnia and van Groesen, 2014a].

The bathymetry is presented in Fig. 3.4 the water depth varies from $0.4 \mathrm{~m}$ in the deeper region to $0.1 \mathrm{~m}$ over the top of the bar. In the experiment the wave height is measured at seven positions: S1, S2, $\cdots, \mathrm{S} 7$. Position S2 is at the upslope area (a 1:20 slope), positions S3, S4 are at the top of the bar and positions S5, S6 are at the downslope area (a 1:10 slope) and position $\mathrm{S} 7$ at the flat bottom close to 


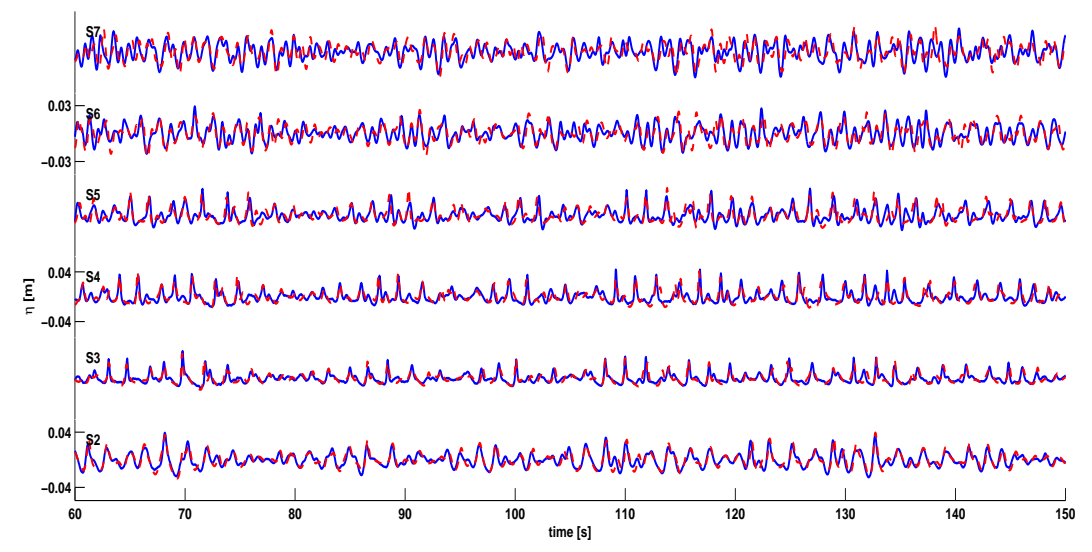

Figure 3.5: Elevation time traces at positions S2, S3, $\cdots$, S7 are shown for the measurement (blue, solid-line) and the simulation (red, dashed-line) of the irregular breaking wave propagating over a bar.

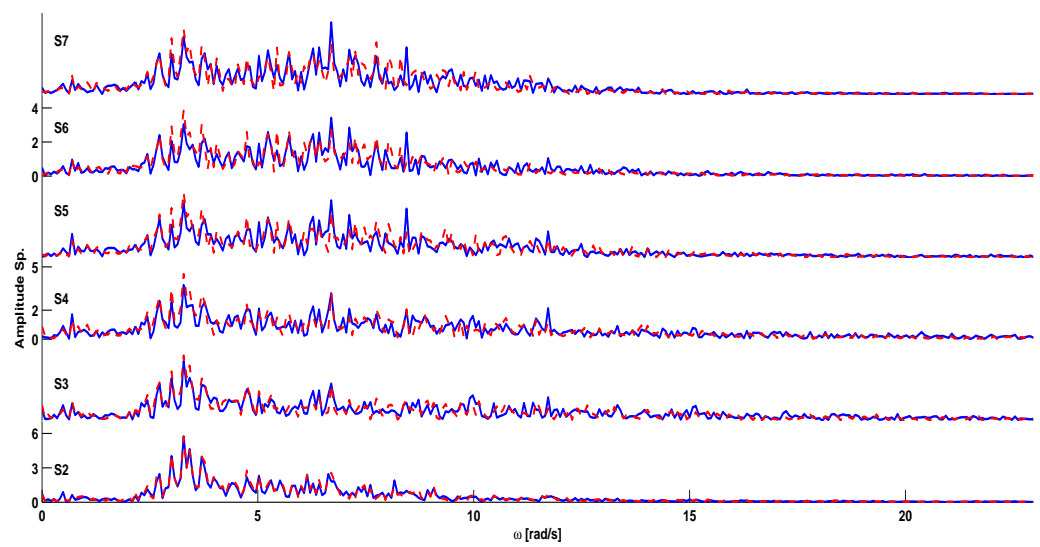

Figure 3.6: Corresponding spectra of the time traces in Fig. 3.5.

the downslope area. The measured surface elevation at S1 at the foot of the slope is used as influx signal for our simulation.

In Fig. 3.5 we show the elevation time traces at all measurement positions in the time interval $(60 ; 150) \mathrm{s}$. The simulated surface elevation is in good agreement with the measurement. The wave transformation, the shoaling process (at S2), breaking at the top of the bar (at S3 and S4) and then the wave decomposition (at S5, S6, S7) are all well reproduced. The main discrepancy between measurement and simulation is observed at the downslope area (S5, S6 and S7). The corresponding spectra are shown in Fig. 3.6.

In Fig. 3.7, we show at the top-left the spatial evolution of the maximum tem- 

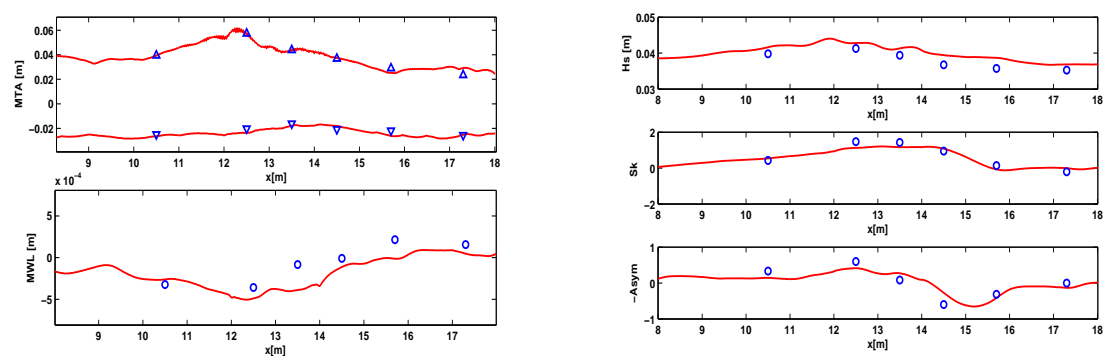

Figure 3.7: Spatial evolution of wave characteristics as computed (red, lines) and measured (blue, symbols) of irregular breaking waves propagating over a bar. At the top-left $\triangle:$ maximum temporal crest, $\nabla:$ minimum temporal trough, at the bottom-left, o: mean water level. At the right, Hs: significant wave height (top), Sk: skewness (middle), As: asymmetry (bottom).

poral crest and the minimum temporal trough (left), at the bottom-left the mean water level; at the right, the significant wave height (top), skewness (middle) and asymmetry (bottom). The skewness $S k$ measures the crest-trough shape, and the asymmetry $A s$ measures the left-right differences in a wave, defined as:

$$
S k=\frac{\left\langle(\eta-\bar{\eta})^{3}\right\rangle}{\left\langle(\eta-\bar{\eta})^{2}\right\rangle^{3 / 2}} \quad A s=\frac{\left\langle\mathbb{H}(\eta-\bar{\eta})^{3}\right\rangle}{\left\langle(\eta-\bar{\eta})^{2}\right\rangle^{3 / 2}}
$$

where $\bar{\eta}$ is the wave-averaged surface elevation, \langle\rangle is time averaging operator and $\mathbb{H}$ the Hilbert transform. The plots show that the simulation captures the main wave characteristics in a good way.

\subsubsection{Harmonic breaking wave running up a coast}

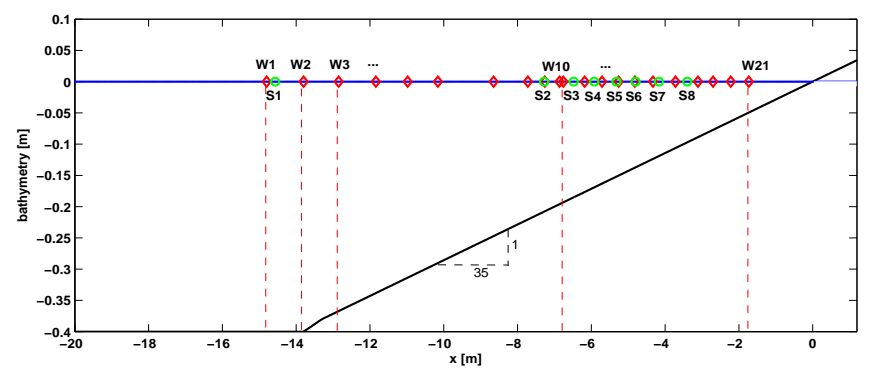

Figure 3.8: Lay out of the experiments of [Tinq and Kirby, 1994]. The location of the wave gauges for surface elevation are indicated by $\diamond$ (red). The interior horizontal velocity is measured at 8 positions, indicated by $\circ$ (green), every $0.01 \mathrm{~m}$ in the vertical direction.

In this section we show simulation results of a harmonic wave running up a coast. Except for the surface elevation also the interior horizontal velocity is compared to 

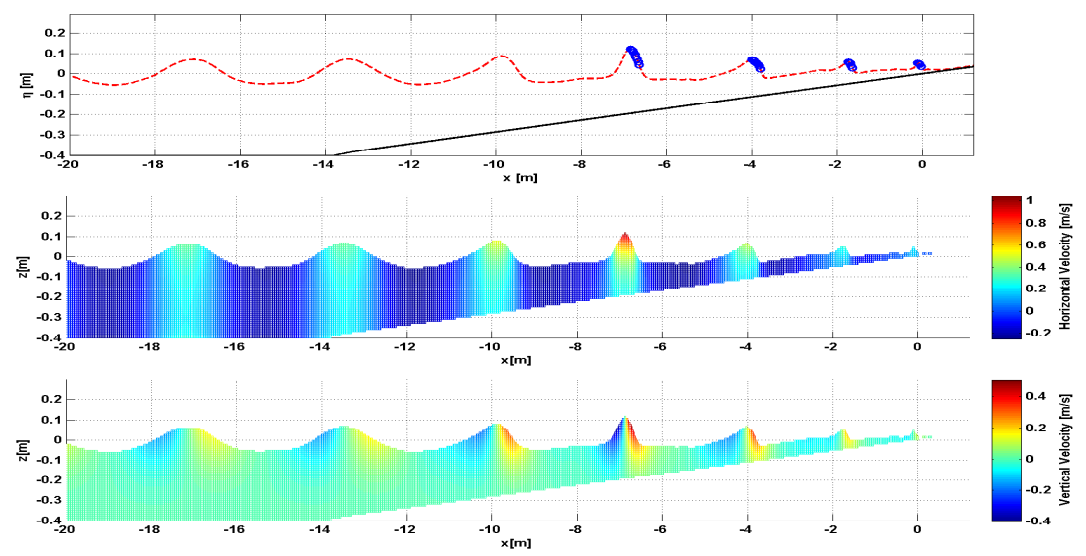

Figure 3.9: Simulation results of breaking waves during run-up; shown are the spatial surface elevation (top), the interior horizontal velocity (middle) and the interior vertical velocity (bottom) at $t \approx 60 \mathrm{~s}$. Breaking nodes are indicated by circles (blue).

data of laboratory experiments conducted by Ting and Kirby [1994]. Harmonic waves were generated above a flat bottom with depth $0.4 \mathrm{~m}$ and propagate over a 1:35 sloping coast. In this section we show only the spilling breaker case, for which the wave period is $T=2.0 \mathrm{~s}$ and the incident wave height $H=0.125 \mathrm{~m}$.

The bathymetry is presented in Fig. 3.8, indicating 21 positions of wave elevation measurements and 8 positions of interior horizontal velocity measurements, measured every $0.01 \mathrm{~m}$ in the vertical direction.

Fig. 3.9] shows results of the simulation: the spatial surface elevation, the interior horizontal velocity and the vertical velocity at $t \approx 60 \mathrm{~s}$. Breaking in the simulation starts at $x \approx-6.9 \mathrm{~m}$ which agrees with the measurement, and then continues till the shore. The initiation of breaking in the simulation was obtained by the kinematic criterion $U / C>=0.55$ in which $U$ is the crest speed and $C$ is the phase speed at the crest. The corresponding interior horizontal velocity has maximum value in the crest when the breaking starts. Correspondingly, the interior vertical velocity has maximum value in the front of the wave.

Fig. 3.10 at the left shows time traces of surface elevation of simulation and measurement at W1 (deep area) to W21 (close to the shore). The wave shape is well reproduced and in phase during shoaling and breaking which takes place at W10. At positions close to shore, the simulation overestimates the wave crest as shown also in the maximum temporal crest plot and skewness plot in Fig. 3.10. However the simulation captures the main wave characteristics in a quite good way.

Fig. 3.11 shows the variations of the time-mean horizontal velocity at different depths at several locations indicated in Fig. 3.8. The time-mean horizontal velocity $\bar{u}$ is normalized by the wave celerity $(C=\sqrt{g D})$. In agreement with the measurement, the calculated time-mean horizontal velocity is in the onshore direction near the surface and in the offshore direction below the trough level. The main discrepancy between the calculated and the measured value is at positions close to the shore. 

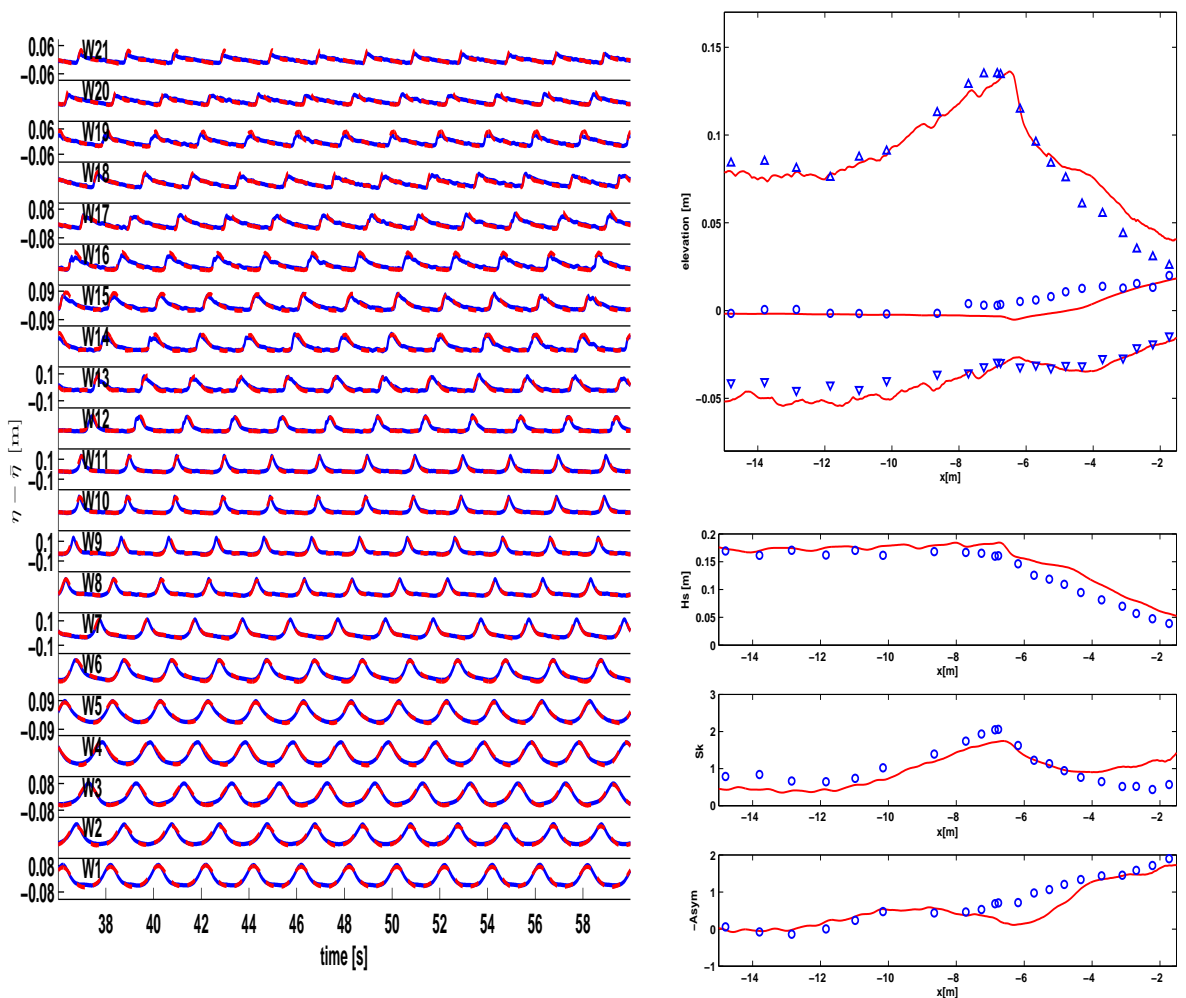

Figure 3.10: For the harmonic breaking wave running up a coast, at the left the elevation of simulation and experiment as in Fig. 3.5 and at the right same information as in Fig. 3.7 .

\subsubsection{Wave-wall interactions}

In the first subsection we deal with a uniformly partially reflecting wall, i.e. all waves are reflected with the same fraction $R$. In the second subsection we show results for frequency dependent reflection at the wall.

\section{Fully or partially reflecting walls}

In this section we show simulation of harmonic waves colliding at a fully or partially reflecting wall. The simulation results are compared with analytic solutions.

The relation between the reflection coefficient $R$ and the constant $\rho$ in Eq. 3.20 is confirmed by simulation results as shown in Fig. 3.12. The simulation results are for harmonic waves with period $2 \mathrm{~s}$ which corresponds to wavelength $\approx 6.3 \mathrm{~m}$, initial wave height $0.1 \mathrm{~m}$, propagating above a flat bottom with depth $5 \mathrm{~m}$ and colliding against a wall (at $x=150 \mathrm{~m}$ ), simulated by the linear Hamilton equations and the second order Hamilton equations for different values of $\rho$.

In Fig. 3.13 we show the spatial wave profile at $t=522.2 \mathrm{~s}$ and time trace at 

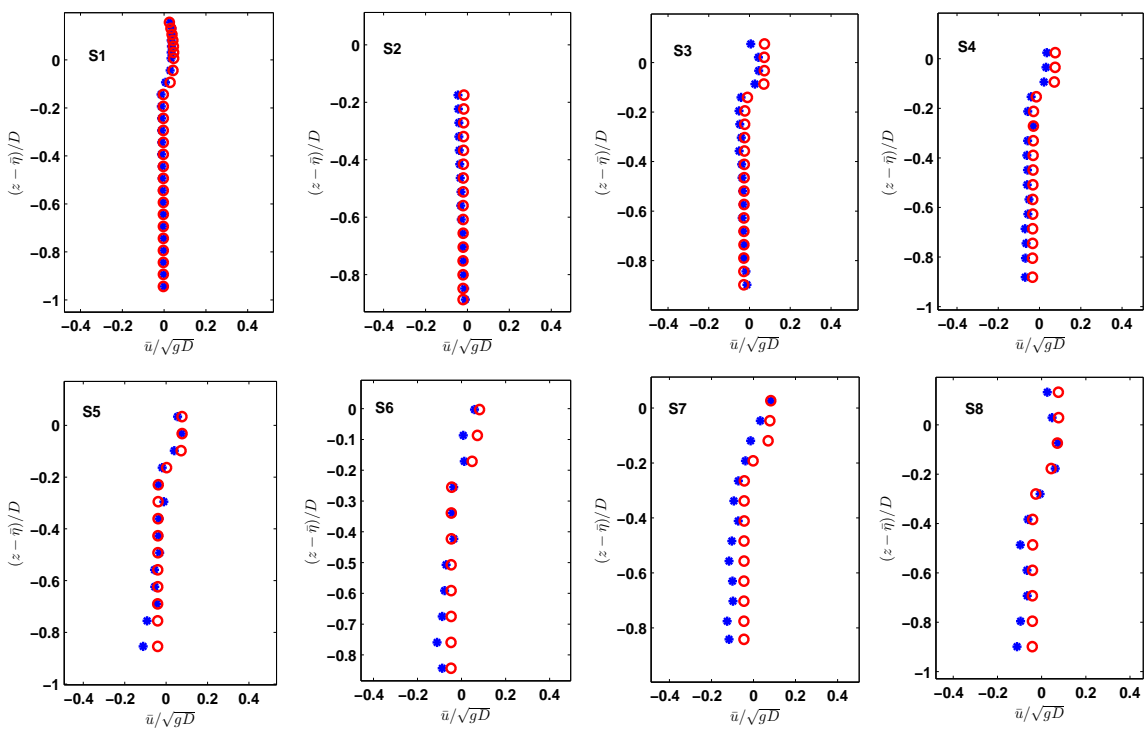

Figure 3.11: Shown are the time-mean horizontal velocity of calculation $\circ$ (red) and measurement * (blue) below horizontal spatial points S1, S2 $\cdots$, S8 illustrated in Fig. 3.8.

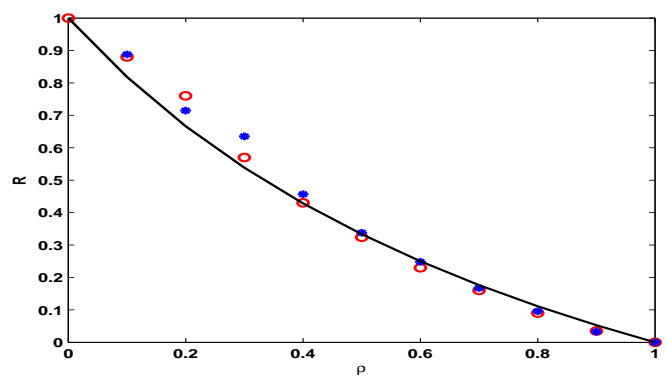

Figure 3.12: Relation between the reflection coefficient $R$ and $\rho$. Simulation results by the linear Hamilton equations $\circ$ (red), the nonlinear Hamilton equations * (blue) are compared to the exact relation Eq. 3.20 (solid-line, black).

the wall position $x=150 \mathrm{~m}$ of the linear simulation of the harmonic waves with period $2 \mathrm{~s}$. It shown that the reflected wave height is as desired 2 times the initial wave height for full reflection and 1.5 times for $50 \%$ reflection. In Fig. 3.14 we show the nonlinear simulations with the same initial wave characteristics. The nonlinear effects lead to a small modulation in the wave elevation.

Fig. 3.15 shows linear simulations with different wave periods $4 \mathrm{~s}$ and $6 \mathrm{~s}$. The harmonic waves with period $4 \mathrm{~s}$ corresponds to wavelength $\approx 22.2 \mathrm{~m}$ and period $6 \mathrm{~s}$ corresponds to wavelength $\approx 38.1 \mathrm{~m}$. These are long wave compared with the depth of $5 \mathrm{~m}$. The reflected wave height is approximately 2 times the initial wave height for full reflection; the wave with period $6 \mathrm{~s}$ shows a slightly higher amplitude at the 

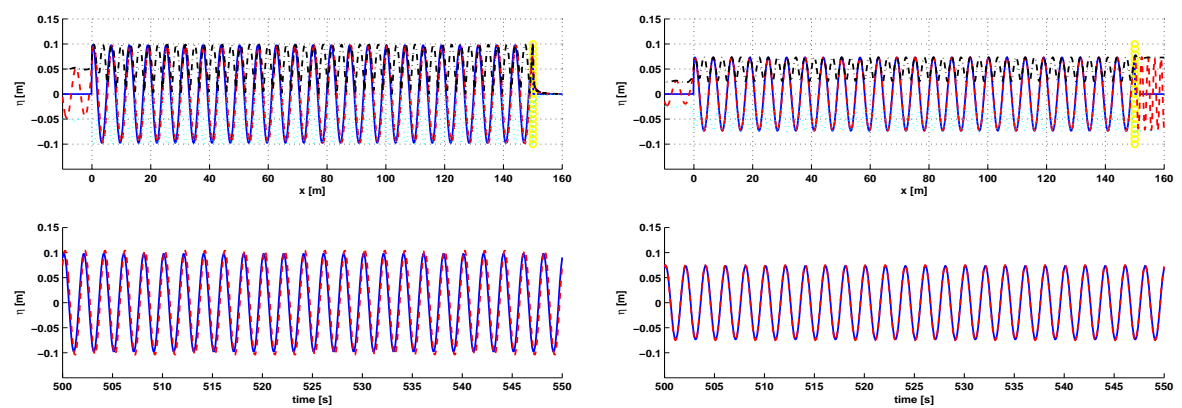

Figure 3.13: Reflected harmonic wave profiles at $t=522.2 \mathrm{~s}$ (at the top) and time trace at the wall position (at the bottom) for waves with period $2 \mathrm{~s}$ with $\lambda / D=1.3$. At the left and at the right are simulation results for $100 \%$ and $50 \%$ reflecting wall, respectively. Simulations using the linear Hamilton equations are indicate by dashed-line (red), the linear analytic solution by solid-lines (blue), the maximum temporal crest by dashed-dot (black), the minimum temporal trough by dots (cyan) and the wall at $x=150 \mathrm{~m}$ by vertical line (yellow)
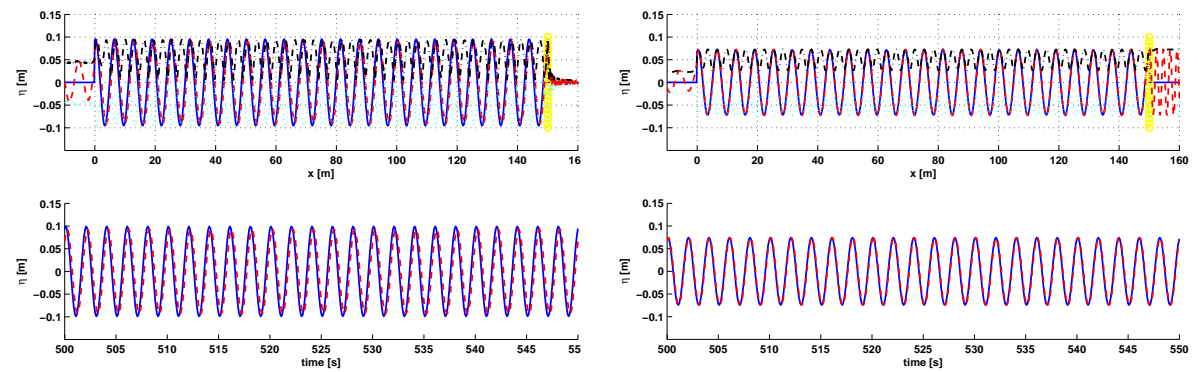

Figure 3.14: Same as in 3.13. Now for simulations (dashed-line, red) using the the second order Hamilton equations compared to the linear analytic wave (solid-line, blue)
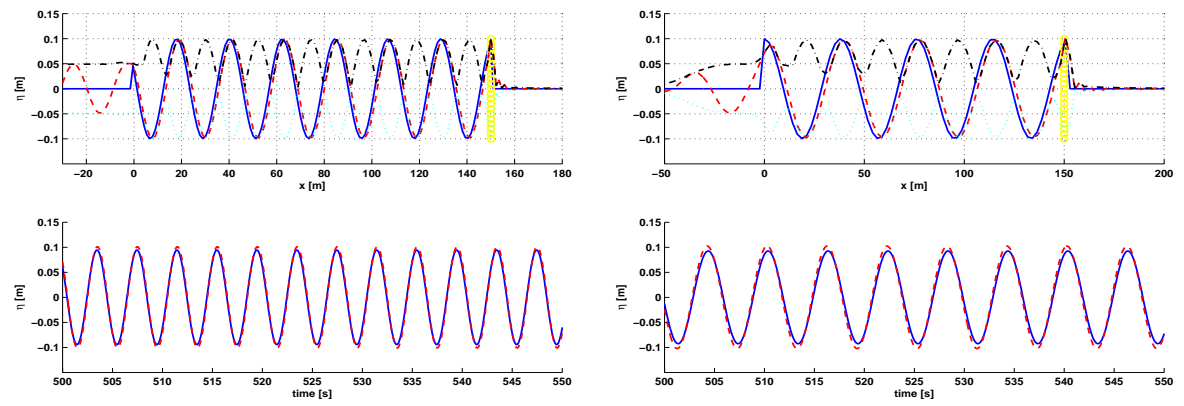

Figure 3.15: Same as in 3.13, Now for simulations with period $4 \mathrm{~s}$ (left) and $6 \mathrm{~s}$ (right) corresponding to $\lambda / D=4.4$ and 7.6 respectively. 
wall while small shifts in phase are also observed.

\section{Frequency dependent reflecting wall}
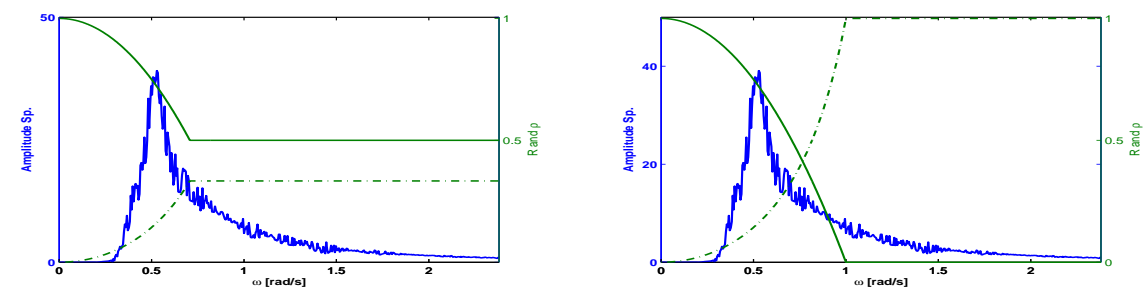

Figure 3.16: Shown are the spectrum of the influx signal (solid-line, blue), the prescribed reflection coefficient $R(\omega)$ (solid-line, green) and the calculated $\bar{\rho}(\omega)$ (dashed-dot, green). At the left, $\bar{\rho}_{1}$ for $R_{1}=1-\min \left(\omega^{2}, 0.5\right)$, and at the right, $\bar{\rho}_{2}$ for $R_{2}=1-\min \left(\omega^{2}, 1\right)$.
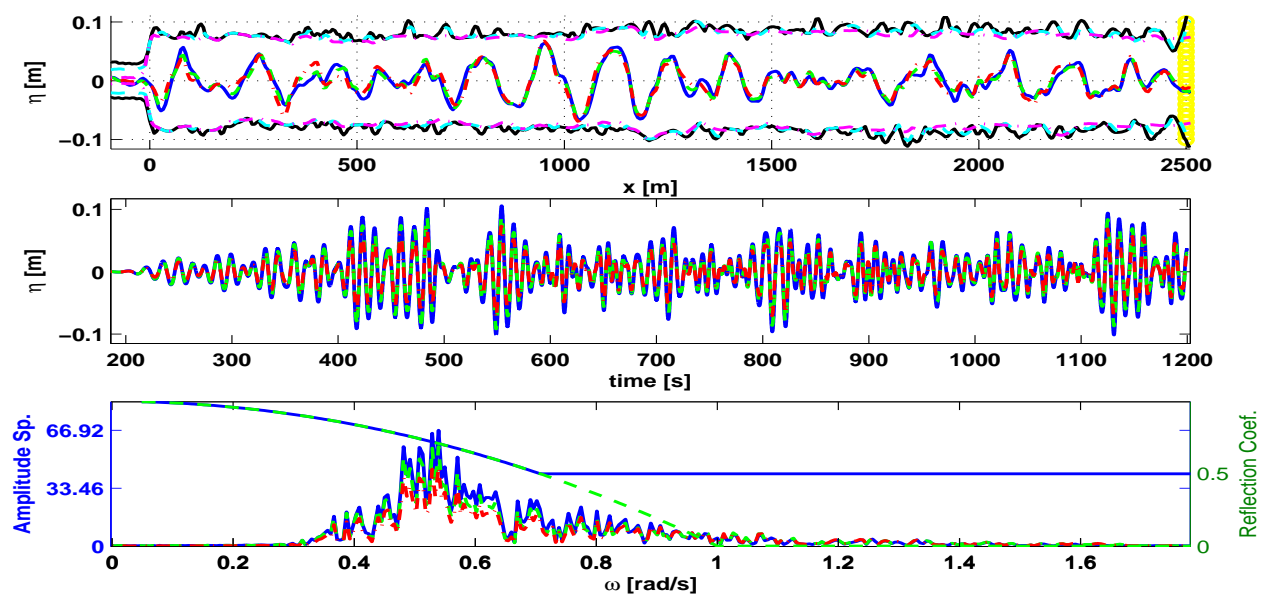

Figure 3.17: Shown are simulation results with the reflection coefficient $R_{1}$ (blue, solidline), $R_{2}$ (green, dashed-line) and without wall (red, dashed-dot). At the top, the wave profiles at time $t=1000 \mathrm{~s}$ and maximum temporal amplitudes for: simulation with the wall (black, solid-line for $R_{1}$ and cyan, dashed-line for $\left.R_{1}\right)$, without wall (magenta, dashed-dot) and the wall at $x=2500 \mathrm{~m}$ (yellow, vertical line). The middle plot shows time traces at the wall position. The bottom plot shows the spectrum at the wall position with at the left axis the amplitude spectra, and at the right axis the value of the reflection.

In this section we show simulation results of waves colliding at a frequency dependent reflecting wall. The waves are irregular waves with JONSWAP spectrum with gamma 3.3, significant wave height $0.1 \mathrm{~m}$, peak period $12 \mathrm{~s}$ above a flat bottom with depth $25 \mathrm{~m}$. We show comparison of the second order nonlinear simulation with and without wall, for walls with two reflection properties $R_{1}=1-\min \left(\omega^{2}, 0.5\right)$ and $R_{2}=1-\min \left(\omega^{2}, 1\right)$ as shown in Fig. 3.16. 
Fig. 3.17 shows that with $R_{1}$ the simulation shows the correct full reflection of long waves and partial reflection $R \in[0.5,1)$ for shorter waves. With $R_{2}$ the simulation shows partial reflection with $R \in(0,1]$ for $\omega<1$, and full transmission of shorter waves.

\subsubsection{Dam-break problem}
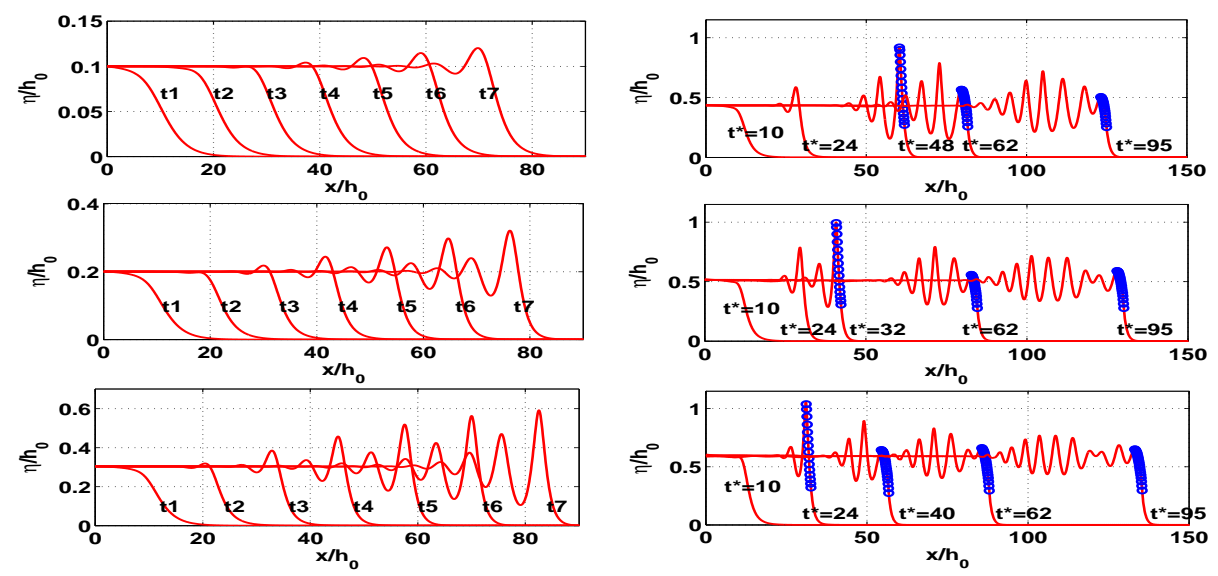

Figure 3.18: At the left are shown spatial surface elevations for undular bore simulations with initial height $\left(\eta / h_{0}\right) 0.1$ (top), 0.2 (middle), 0.3 (bottom) at various times: $t 1=10$, $t 2=20, t 3=30, \cdots, t 7=70$. At the right are shown the elevations for breaking bore simulations with initial height 0.43 (top), 0.52 (middle), 0.6 (bottom); the breaking nodes are indicated by $\circ$ (blue).

In this section we simulate undular bores propagating into constant-depth, still water. This case is also known as the dam break problem that has been widely studied as a standard illustration of competing effects of dispersion and nonlinearity.

In this testcase we reproduced and extended simulations of previous work by Wei et al. 1995]. The initial conditions describe a gentle transition between a uniform flow and still water

$$
u=\frac{1}{2} u_{0}[1-\tanh (x / a)], \quad \eta=u+\frac{1}{4} u^{2} .
$$

Here $\eta$ is the surface elevation and $u$ is the velocity. In our models the velocity is the tangential velocity, which is different from the horizontal velocity in Wei et al., 1995]. $u_{0}$ is the velocity of the uniform flow from the left boundary and $a$ is a number sufficiently large so that the initial motion can be described by Airy's theory. The same value $a=5$ is used and $u_{0}$ is chosen so that the surface elevation at the left boundary is as required $\eta_{0}=0.1,0.2,0.3$. The simulations as shown in Fig. 3.18 at the left are presented in the same scale as in Fig. 7 of Wei et al., 1995], $t^{*}=t / \sqrt{h_{0} / g}, x^{*}=x / h_{0}$ with $h_{0}=1$. Qualitatively the simulations using the Hamilton equations are similar with the simulations using the fully nonlinear potential flow presented in Fig. 7 [Wei et al., 1995]. 
To simulate more extreme cases, we increased the initial surface elevation to values $\eta_{0}=0.43 ; 0.52 ; 0.6$ that lead to breaking bores, shown in Fig. 3.18 at the right. The bores start to break at time $t^{*}=24,32$ and 40 at different positions for the simulations with three different initial conditions respectively. The waves are shown to break at the front.

\subsection{Conclusion}

It is quite remarkable that the method proposed here, the rather straightforward generalization given in Eq. 3.12 of the explicit expression of the phase velocity from Airy's linear theory, leads to a model and implementation that performs so well for even the most difficult cases as shown in Section 3.4 .

A major reason must be that the model has the exact asymptotic behaviour of the limit of linear waves above flat bottom, as well as the shallow water limit, both limits valid for all types of waves i.e. for all wave lengths; this despite the strict conclusion about the second order accuracy from the classical 'order' reasoning as presented in Section 3.2.3 which may be too pessimistic for the model that is inherently based on a non-algebraic formulation.

Provided the Fourier Integral Operators are correctly dealt with, in particular the symmetry properties, the Hamilton equations are obtained in a very explicit way. Consequently, the actual numerical implementation is very simple: using FFT's for the spatial discretization and an explicit time solver, the explicit formulas are literally copied without any adjustments or 'trics'.

For computational effectiveness, the interpolation of the symbols in the FIO's is essential; in all cases considered so far (including those in this paper), just 2 or 3 interpolants are enough to get accurate results. As a consequence, most simulations can be done in (essentially) less than $25 \%$ of the physical time in environmental geometries.

With the methods described in this paper in Section 3.3 we have overcome the localization problems that are typical for global methods such as Fourier methods. At this moment similar methods are used to model and simulate wave-ship interactions, results of which will be published elsewhere. Although the simulations are performed in a dimension reduced way, the calculation of the internal flow as described in Section 3.2.5 will be useful for various applications.

Simulations as shown in this paper can be performed with HAWASSI-AB software. 


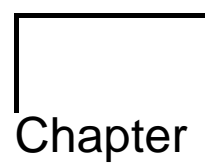

\section{4}

\section{Design of wave breaking experiments and a-posteriori simulations}

\section{Summary}

In this chapter, we present results of 30 wave breaking experiments that were designed by HAWASSI-AB software. The experiments were carried out in a wave tank of Technical University of Delft (TUD). The use of the efficient simulation code can optimize the experiments by designing the influx such that waves will break at a predefined position. The consecutive actual measurements agree well with the numerical design of the experiments. Using the measured elevation close by the wave maker as input, the software recovers the experimental data in great detail, even for rather short (up to $L / D=1$ ) and very steep breaking waves with steepness parameter $(a k)$ till 0.4 .

\subsection{Introduction}

For the design of fixed or flexible structures in the coastal area such as wind mills and oil platforms, knowledge of expected forces in extreme waves are of paramount importance. Experiments in wave tanks are often used to obtain impact data in well-controlled circumstances. Since the interest is mainly in extreme wave conditions, it is required to prepare the incoming wave such that the desired extreme behaviour takes place at the defined position of the structure. Limitations on the wave generator and the complicated distortions during the wave propagation towards the structure makes this a challenging task.

\footnotetext{
${ }^{1}$ The contents of the chapter is partly presented as a proceeding article Kurnia et al., 2015] and an internal report Kurnia and van Groesen, 2015a
} 
This chapter presents results of 30 wave breaking experiments conducted in the long wave tank of TU Delft, Department of Maritime and Transport Technology (6,7 and 10-12 March 2014). Simulations performed before the experiment to determine the required wave maker motion and a-posteriori simulations that use a measured time trace as influx for calculation further downstream are also presented.

The 30 different experiments cover a broad range of breaking waves of various types. Those are roughly grouped together as follows: 11 focussing waves, 7 bichromatic wave trains, 9 irregular waves, 2 cases of 'soliton on finite background', 1 harmonic wave with added focussing wave.

Characteristic for all cases is the rather broad spectrum (although restricted by wave maker properties). In each group, the cases differ in amplitude, period and steepness. The range of wavelength for these cases runs from 1 to 4 times the depth and the steepness parameter $(a k)$ till 0.4. 27 experiments showed breaking as designed; the harmonic focussing case, and the two test cases TUD1403Ir7 and TUD1403Foc12 were (designed to be) non-breaking.

The aim of this Chapter is to show that HAWASSI-AB, a Hamiltonian Boussinesq model with breaking mechanism, is sufficiently accurate to support the design task prior to the actual experiment. Moreover, the a-posteriori use of the measured elevation close to the wave maker to initiate the simulation, leads to reconstruction of the waves that is accurate at all measurement positions.

The organization of this chapter is as follows. Section 4.2 describes the laboratory experimental set-up. Section 4.3 gives a brief description of the simulation model. In Section 4.4, comparison of measurement results with the a-priori and a-posteriori simulation will be presented. Conclusions will finish this chapter.

\subsection{Experimental set up}

The wave tank at Technical University of Delft was used to perform the experiments. The tank is $142 \mathrm{~m}$ long, $4.22 \mathrm{~m}$ wide and the depth during the experiment was 2.13 $\mathrm{m}$. At the end of the tank, waves are absorbed by an artificial beach. Waves are generated by a flap type wavemaker. Fig. 4.1 shows a cross-section of the wave tank.

Resistance type of wave probes were used to measure surface elevation at various positions at the centre-line of the tank. The wave probes operate by measuring the current that flows between two stainless steel wires that are immersed in the water. This current is converted to an output voltage that is directly proportional to the immersed depth.

Calibration of a potentiometer on the wavemaker and the wave probes in the fluid has been done before the start of the experiments. This calibration has been used to determine the transfer function for optimized performance.

The same measurement positions were used for a total of 30 different experiments. The experiments were designed in such a way that most breaking waves could be caught at the same position, which then were selected as the measurement positions.

The position of breaking of the waves did not always coincide precisely with the wave probe positions. Nevertheless, from the recorded video and markers in 


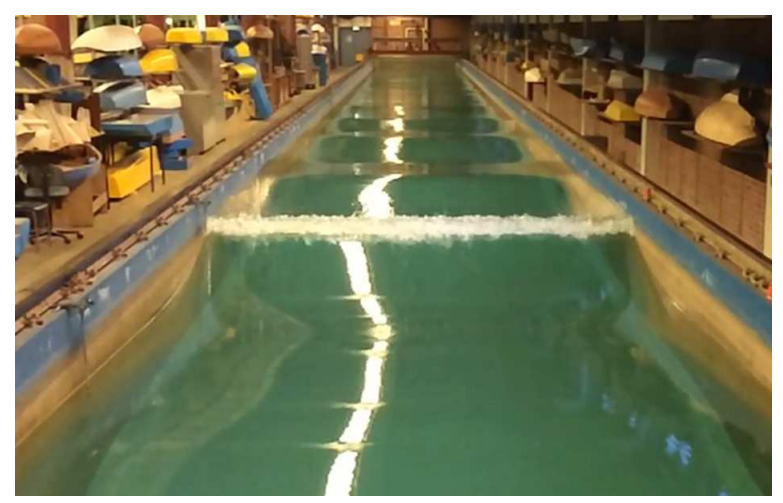

Figure 4.1: The wave tank of TUD. View from a carriage at approximately $70 \mathrm{~m}$ to wave maker.

the wavetank, the breaking position and breaking moment could be approximated afterwards. Comparison of observed and simulated breaking events will be shown in the next section on design and reconstruction.

A schematic lay-out is shown in Figure 4.2. The elevation is measured at positions $\mathrm{W} 1$ at $x=10.31 \mathrm{~m}, \mathrm{~W} 2$ at $x=40.57 \mathrm{~m}, \mathrm{~W} 3$ at $x=60.83 \mathrm{~m}, \mathrm{~W} 4$ at $x=65.57 \mathrm{~m}$, W5 at $x=70.31 \mathrm{~m}$ and W6 at $x=100.57 \mathrm{~m}$.

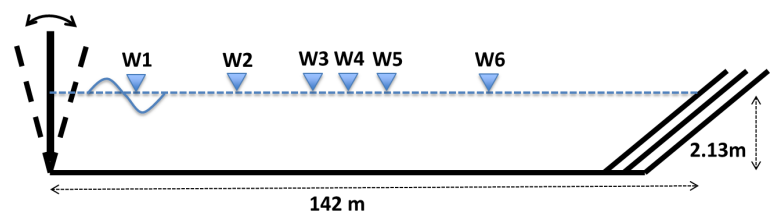

Figure 4.2: Layout of the experimental set-up.

In Appendix A.1 an overview of the experiments is given with all main wave parameters.

\subsection{Simulation model}

The model used for the numerical simulations is part of HAWASSI-AB, the Hamiltonian Wave Ship-Structure Interaction, using Analytic Boussinesq model. For the present cases only the wave facility has been used.

A full description of the code and the breaking model can be found in Kurnia and van Groesen, 2014a . The most characteristic properties of the model are now summarized.

The model is of Boussinesq type, which means that the interior fluid motion is not calculated but modelled so that a spatial reduction is obtained.

The model has a Hamiltonian structure, with main consequence that it is exactly energy conserving for non-breaking waves. 
The main disadvantage of most Boussinesq equations is overcome by using a spatial-spectral implementation so that dispersion is exact for all wave lengths in the order of nonlinearity of the equation.

For the experiments in this chapter, the third-order equation (ABHS3 in Kurnia and van Groesen, 2014a]) has been used.

Wave generation is done in an embedded way by influxing the given elevation signal at the influx position through a source in the continuity equation; in Lie, 2013] this generation is described in detail. Here area influxing has been used with an adaptation area to prevent the generation of spurious waves for the extreme influx heights used in some of the experiments.

For breaking the mechanism as proposed by Kurnia and van Groesen [2014a] is used; the initiation of breaking is determined by a kinematic breaking criterion.

Instead of the absorbing beach of the wave tank, the simulated wave is smoothly damped to avoid any reflections from the end.

Simulations with the HAWASSI-AB code are reasonably fast; depending on the specific case, computation times are 0.5 to less than 3 times the physical time. The simulations are also robust for change of parameters such as grid size, initiation of breaking, etc. as shown in Kurnia and van Groesen, 2014a].

\subsection{Design and reconstruction}

Of the 30 experiments listed in Appendix A.1 we select a few characteristic cases for presentation here. A full account of all simulations is available in Appendix A.2 and A.3.

In the first subsection we compare measurement results with the a-priori simulated wave; we call this the design case. It will be seen that there are some discrepancies between the designed waves and the experiments, but the differences are for most practical applications acceptable. In the second subsection we compare the measurements with the simulated waves that use the measured elevation at W1 as input for simulation; we call this the reconstruction. These simulation results compare better with the experiments. This indicates that the transfer function used to translate information from the designed waves to the wave maker motion must account for the differences.

In all cases, the waves were approximated with an analytic model choosing the steepness parameters such that breaking could be expected. Then the simulation using HAWASSI-AB gave a complete overview of the designed waves including the positions of breaking.

In the following plots of wave elevations, measurements are indicated by blue (solid) lines, and simulations by red (dashed) lines.

\subsubsection{Design cases}

To determine input for the wavemaker, the elevations signal at $10 \mathrm{~m}$ of the a-priori simulated wave has been taken. Using the transfer function of the the wavemaker, the actual wavemaker motion has been calculated. The range of frequencies for the 
input of the wave maker is $(2.4,6)[\mathrm{rad} / \mathrm{s}]$ which restricts the generation of waves to periods in between $1 \mathrm{~s}$ and $2.6 \mathrm{~s}$.

We now describe the results for two cases.

Experiment TUD1403Foc7 is a focussing wave often used in laboratories to generate very high waves as an effect of dispersive wave focussing, in this case with an amplification of about 2.73 .

As shown in Figure 4.3, qualitatively the agreement between design and experiment is good, but differences in the spectra further downstream are quite noticeable at higher frequencies. The breaking position in the design is $65 \mathrm{~m}$ and the observed breaking in the experiment takes place at $59 \mathrm{~m}$. The wave was designed to break at the focussing position but in the experiment the wave breaks one peak wavelength before the focussing.
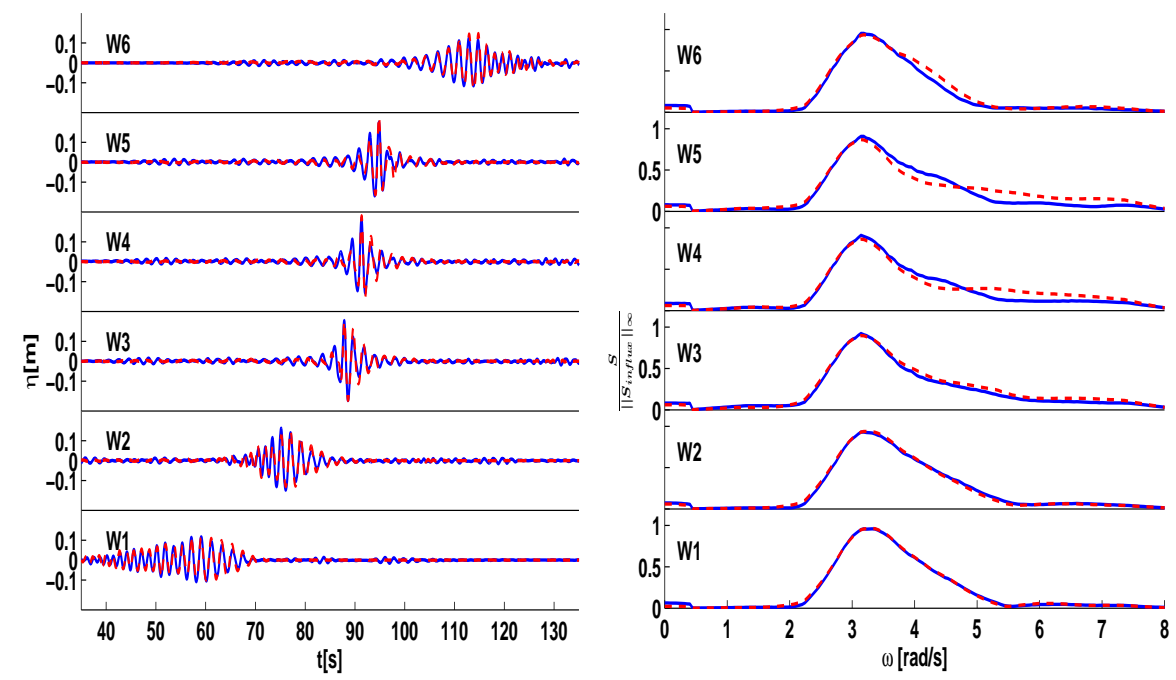

Figure 4.3: Elevation time traces (left) and normalized spectra (right) of TUD1403Foc7 at positions $W 1, \cdots, W 6$ are shown for the measurement (blue, solid) and for the simulation (red, dashed dot).

Experiment TUD1403Bi3 is a bichromatic wave; for specific parameters see Appendix A.1 Severe breaking takes place at multiple positions. Figure 4.4 shows plots of wave elevations and spectra. The spectrum shows substantial energy loss due to breaking; spectra and time traces at different positions show a gradual down-shift of the bi-chromatic pattern, with higher order waves developing and disappearing further downstream.

The spectrum of design and experiment agree quite well; the time traces at larger distances differ in the small wave separation regions between the wave groups. Figure 4.5 shows the positions and times of breaking in the simulation (solid dots, red). Breaking in the experiment as observed in reality and in a movie is indicated also (open dots, blue) but the observation of breaking events in the experiment is limited from $x \approx 50 \mathrm{~m}$ to $x \approx 70 \mathrm{~m}$. 

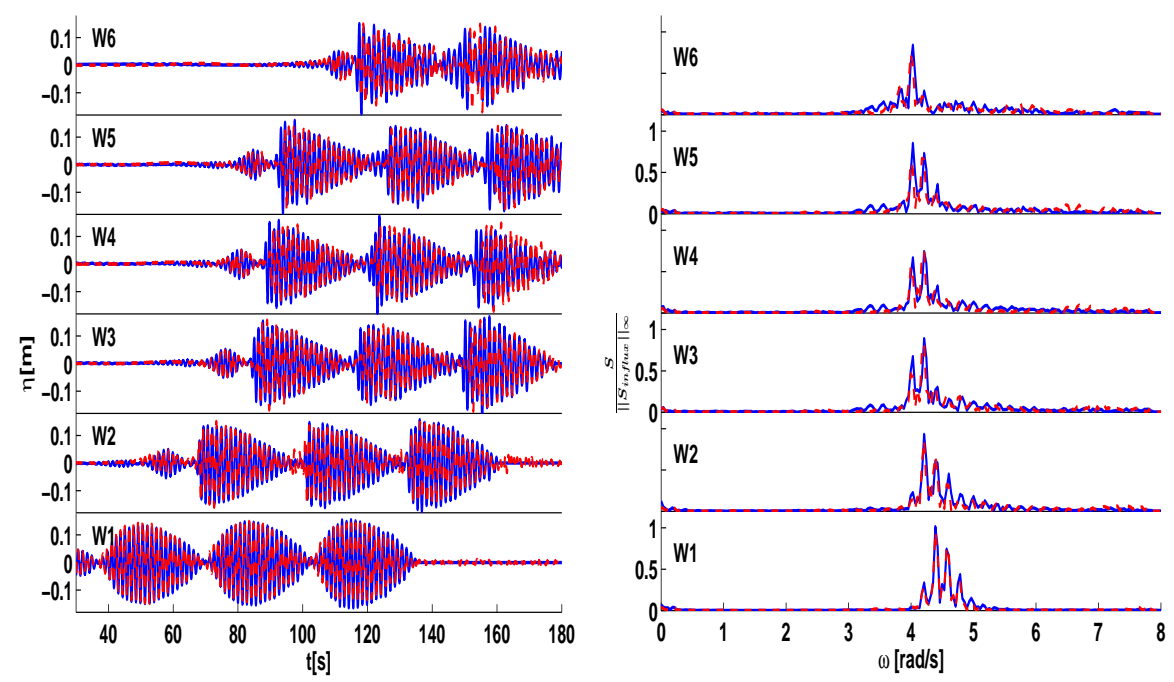

Figure 4.4: Same as Fig. 4.3. Now for TUD1403Bi3.

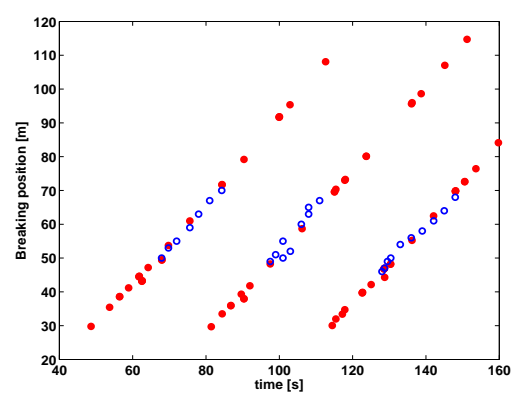

Figure 4.5: Wave breaking positions of TUD1403Bi3 for the simulation (red, solid dots) and the observation in reality and in a movie (blue, open dots).

\subsubsection{Reconstruction cases}

We now reconstruct the experiments by taking the measured elevation at W1 as input signal for the numerical code.

Experiment TUD1403Foc7 This focussing wave with $k a \approx 0.11$ shows single wave breaking. The elevation time traces and the spectra are well reconstructed as shown in Figure 4.6. The differences in the spectra further downstream are rather small. The wave breaks at $x=59.2 \mathrm{~m}$ in the simulation and at $x \approx 59.5$ in the experiment.

Experiment TUD1403Ir10 This irregular wave with $k a \approx 0.27$ shows successive wave breaking for a few waves. During propagation the spectrum becomes slowly broader, with limited energy decay. The spectra and time traces comparisons are shown in Figure 4.7 and the breaking positions in Figure 4.8 . 
Experiment TUD1403Bi3 This bichromatic wave shows abundant continued wave breaking of the steep waves with $k a \approx 0.3$. The elevation time traces and the spectra are well reconstructed as shown in Figure 4.9. Observe the substantial down-shift in the spectrum which may be caused by the breaking, and the substantial energy dissipation. The breaking position of simulation and the limited observation are shown in Figure 4.10 .
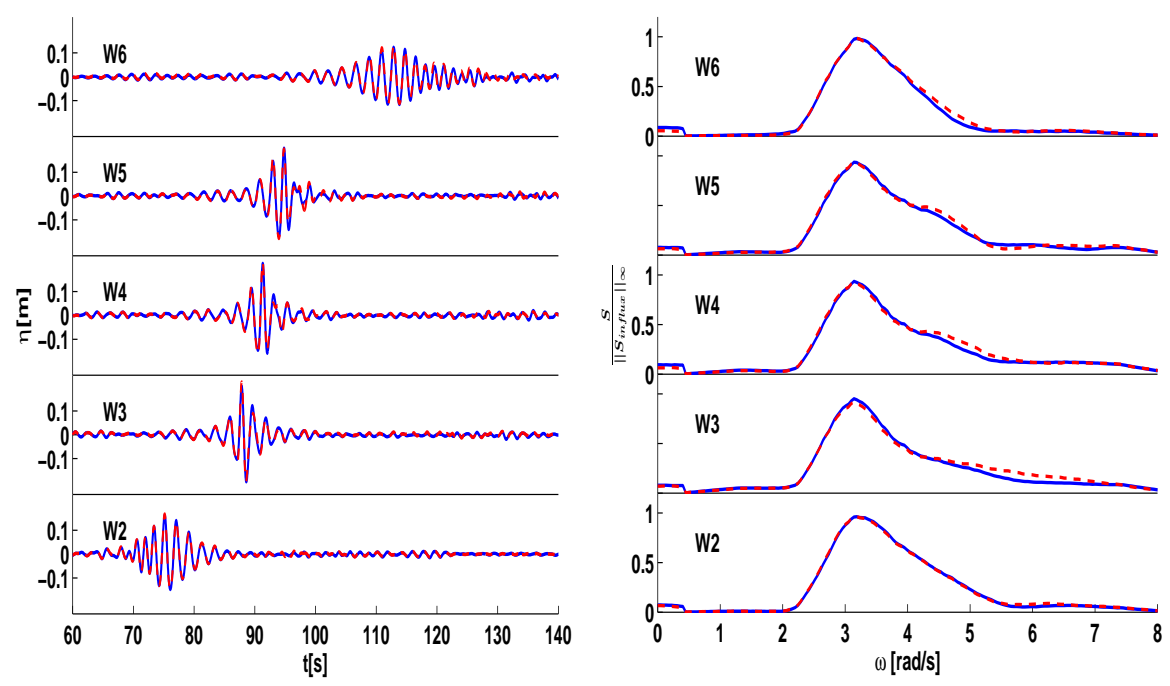

Figure 4.6: Same as Fig. 4.3. Now for reconstruction.

\subsection{Conclusions}

The use of an accurate numerical code can optimize the experiments. A comparison of the design simulations prior to the experiment and the measurements show that the time traces at the measurement positions are quite accurate; the substantial changes in the measured spectra at the successive positions compare reasonably well with the changes of the prior design simulations. However, there are some discrepancies between the designed waves and the corresponding experiments. In the focussing wave case (TUD1403Foc7), the actual breaking position takes place one peak wavelength in front of the designed position, but the focussing position is the same as designed a priori.

Taking the actual experimental wave elevation time trace at the first measurement point as input for the simulations for reconstruction a posteriori the experiment, both breaking and focussing positions of simulation and measurement are in good agreement. This indicates that the transfer function used to translate information from the designed waves to the wave maker accounts for the differences that were observed in the design cases. 

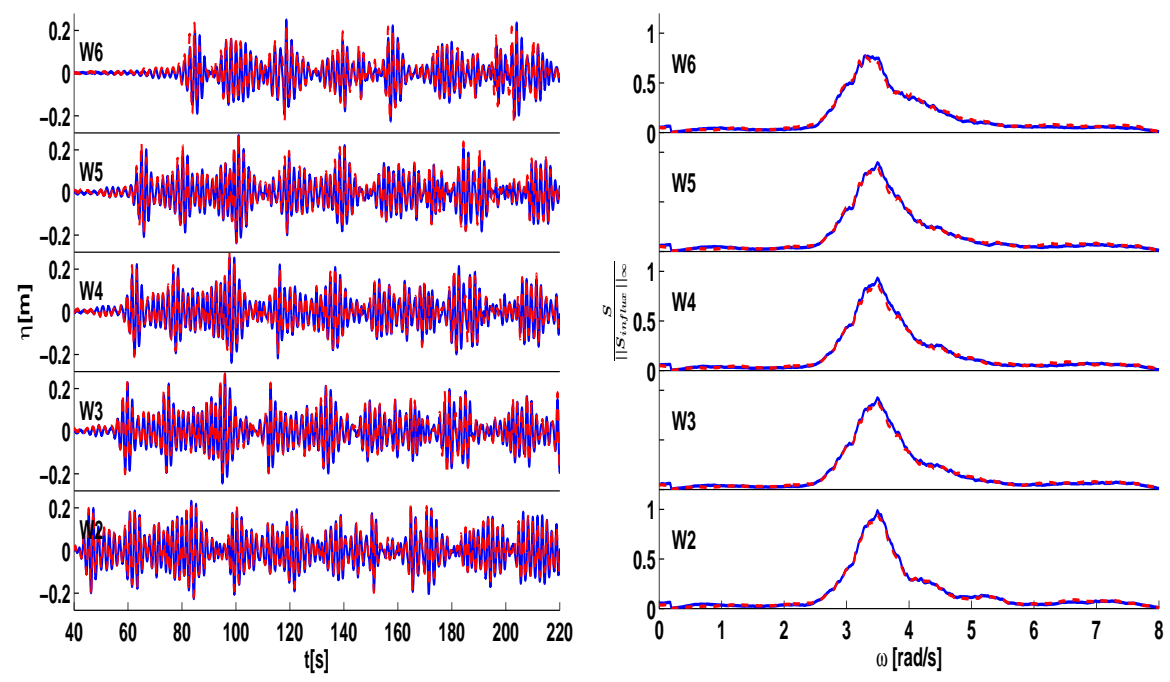

Figure 4.7: Same as Fig. 4.3. Now for TUD1403Ir10 (reconstruction).

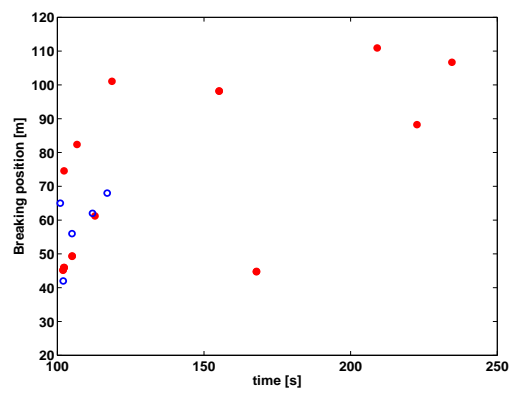

Figure 4.8: Same as Fig. 4.5. Now for TUD1403Ir10 (reconstruction).

The irregular wave case (TUD1403Ir10) showed successive wave breaking for a few waves leading to broadening of the spectrum during propagation with limited energy decay. The simulations showed quantitatively good correlations with measurements. The bichromatic wave case (TUD1403Bi3), showed abundant continued wave breaking of the steep waves leading to the substantial down-shift in the spectrum and the substantial energy decay.

In Table 4.1, we show the correlations at all measurement positions and the relative computation time $(\mathrm{Crel})$. The correlation between the time traces of the measurement and the simulation is defined as the inner product between the normalized signals. Deviations from the maximal value 1 of the correlation measures especially the error in phase, a time shift of the simulation; for correlation - 1 the simulation is in counter phase with the measurement. The relative computation time is defined as the cpu-time divided by the total time of the simulation; the calculations 

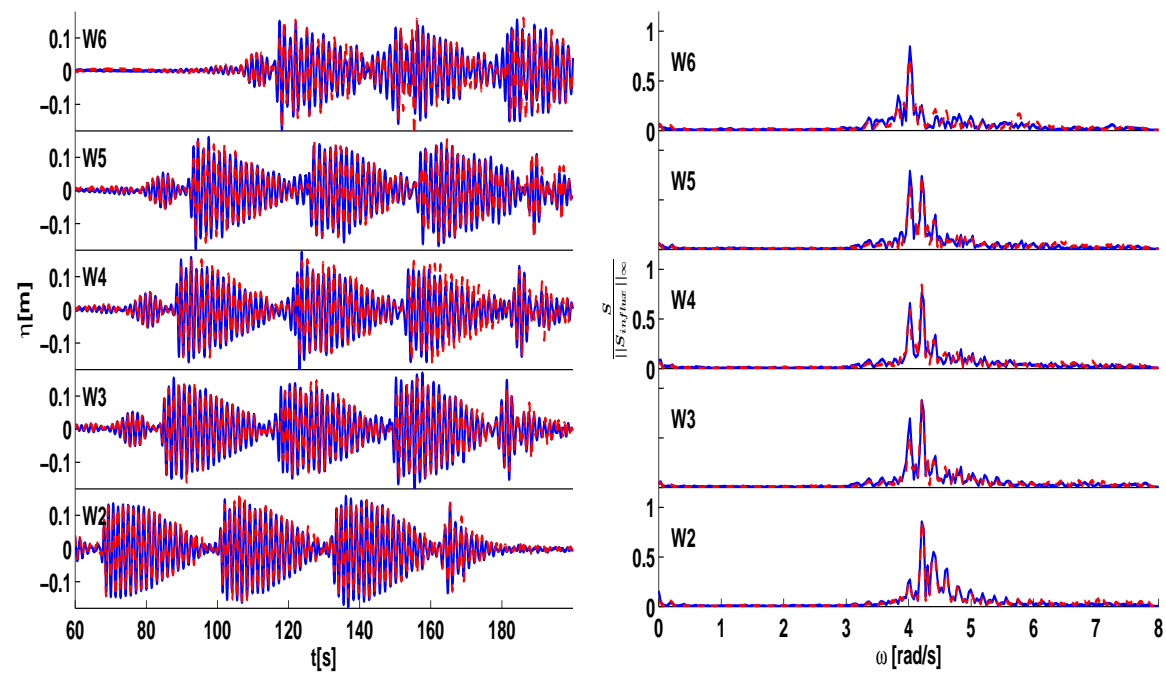

Figure 4.9: Same as Fig. 4.3. Now for TUD1403Bi3 (reconstruction).

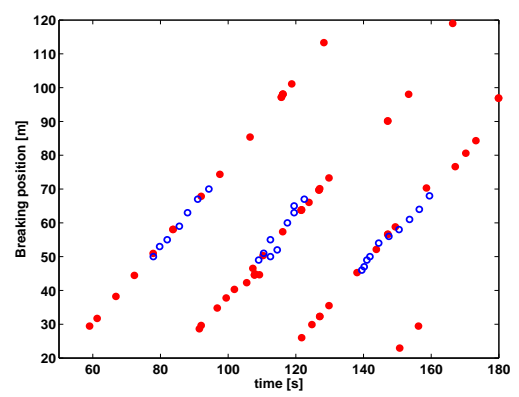

Figure 4.10: Same as Fig. 4.5. Now for reconstruction.

were performed on a desktop computer with $\mathrm{CPU}$ i7, $3.4 \mathrm{GHz}$ processor with 16 GB memory. The simulations show quantitatively good correlations in phase with measurements; and the calculation times rather short. 
Table 4.1: Correlations at measurement positions for the various test cases between simulations and measurements. D stands for the design cases (experiment after simulation) and $R$ for the reconstruction. Crel is the relative computation time.

\begin{tabular}{c|cc|cc|cr}
\hline & \multicolumn{2}{|c|}{ TUD1403Foc7 } & \multicolumn{2}{|c|}{ TUD1403Ir10 } & \multicolumn{2}{c}{ TUD1403Bi3 } \\
& D & R & D & R & D & R \\
\hline W1 & 0.93 & - & 0.94 & - & 0.97 & - \\
W2 & 0.91 & 0.99 & 0.92 & 0.97 & 0.86 & 0.96 \\
W3 & 0.88 & 0.97 & 0.85 & 0.96 & 0.74 & 0.90 \\
W4 & 0.87 & 0.98 & 0.84 & 0.95 & 0.68 & 0.87 \\
W5 & 0.89 & 0.98 & 0.86 & 0.94 & 0.67 & 0.84 \\
W6 & 0.92 & 0.97 & 0.81 & 0.86 & 0.63 & 0.78 \\
Crel & 1.14 & 1.13 & 2.48 & 2.43 & 1.83 & 3.2 \\
\hline
\end{tabular}




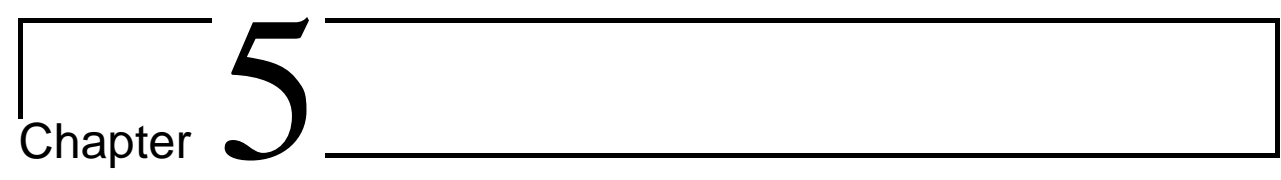

\section{Conclusions and recommendations}

\subsection{Conclusions}

In this dissertation various aspects of water wave modelling are considered. The main topic is the development of an accurate and efficient model that can simulate wave propagation in any range of wave lengths and in any water depth. Moreover, it is desired that the model can deal with various inhomogeneous problems such as bathymetry and walls, leading to wave structure interactions.

A challenge in the study of water waves is that the motion can exhibit qualitative differences at different scales such as deep water versus shallow water, long wavelength versus short wavelength, etc.. That leads to a restriction in the applicability of the existing wave models. It is remarkable that the accurate dispersive wave models, the AB (Analytic Boussinesq) models as presented in this dissertation, showed outstanding performance in terms of efficiency and accuracy. The AB models are uniformly valid for any depth, for large amplitudes and have wide applicability.

A major reason is that the AB models were derived consistently from the Hamiltonian formulation. The approximated Hamilton equations were expressed in the exact phase speed operators with correct order of non-linearity in wave elevation. Thanks to the spatial-spectral implementation the exact dispersion property can be retained without any approximation. The dispersion is essential to obtain highquality wave propagation results. This improves the properties of more classical Boussinesq type of equations. The Hamiltonian consistent approximation guarantees correct evolution of momentum and (approximated) energy.

Furthermore, the AB models can deal with various difficult cases such as breaking or non breaking waves that propagate over a submerged bar or a slope, run-up on the coast, with presence of partially or fully reflective walls, including the dam-break problem. To deal with breaking waves, an extended eddy viscosity breaking model that is applicable for fully dispersive waves was implemented. A kinematic breaking 
criterion that the wave will break when the horizontal particle speed exceeds (a fraction of) the crest speed was used as a trigger mechanism.

To deal with localized effects i.e walls, coasts, submerged bar, etc., a localization method was applied in the global Fourier integral operators that are associated with the nonlinear phase speed operator. It is known that Fourier expansion techniques, different from finite difference or finite element method, lead to some problem when complicated geometric structures need to be included. The inclusion of such fixed structures in a spatial-spectral setting has been shown in this dissertation, it serves perhaps as a first contribution in this topic.

In many applications such as the calculation of wave force on structures requires information of interior properties. A method to calculate or recover the internal flow in the time dynamic or post-processing step of the Boussinesq model has also been shown in this dissertation.

An extensive comparison with (at least 50) laboratory data has been performed. The previous Chapter presented the 30 measurement data of wave breaking experiments in TUD wave tank that were designed using the AB models. It was shown that an efficient and accurate code can optimize wave-tank experiments. Simulations as shown in this dissertation can be performed with HAWASSI-AB software. The demo version can be downloaded on http://hawassi.labmath-indonesia.org.

\subsection{Recommendations}

For further research the following outlook is given:

- In Chapter 3 a generalized AB model was derived. It has been indicated that the model has second order accuracy from the classical order reasoning. This can be improved by starting the derivation from the anzatz of nonlinear extension of the Airy potential and then substituted into the kinetic energy.

- In Chapter 2 the kinematic criterion requires that the wave will break when the horizontal particle speed exceeds (a fraction of) the crest speed. A universal value of this parameter is not known yet. Further investigations on the breaking criterion are much desired.

- The present models aim to simulate long crested waves. The simulation of short crested waves is expected to be a straightforward extension to two horizontal dimensions.

- The method to model wave interactions with fixed structures has been presented. An extension to deal with floating structures such as ships can be done in the future. A Hamiltonian formulation of wave-ship interaction has been derived recently by van Groesen and Andonowati 2015]. 
$\Gamma_{\text {Appendix }} \mathrm{A}$

\section{Supplementary files of the experiments}

This Appendix provides information of the wave breaking experiments that were presented in Chapter 4. The characteristic quantities of all main wave parameters are presented in Section A.1. The comparison between the experiments and apriori simulations is presented in Section A.2 The full comparison between the experiments and a-posteriori simulations is presented in Section A.3.

\section{A.1 The characteristic quantities of the designed waves}

The following table summarises the characteristic quantities of all the designed waves. The successive columns provide the following information: $t$ max: maximum time, Tp: peak period, $(T 0, d t): T 1=T 0+d t / 2, T 2=T 0-d t / 2$ (Bichromatic periods), $\lambda$ p: peak wavelength, kp.a: steepness, H0: maximum wave height of influx signal, MTT: maximum temporal trough, MTC: maximum temporal crest, $\mathrm{H} 1$ : maximum wave height of maximal signal, $\mathrm{H} 1 / \mathrm{H} 0$ : amplification, $\mathrm{xb}$ : successive breaking positions (separated by semicolon), or the range of breaking position as indication of many breaking events (denoted by $\mathrm{x} 1-\mathrm{x} 2$ ), and tb: the corresponding breaking time. The information is based on a-priori simulations. 


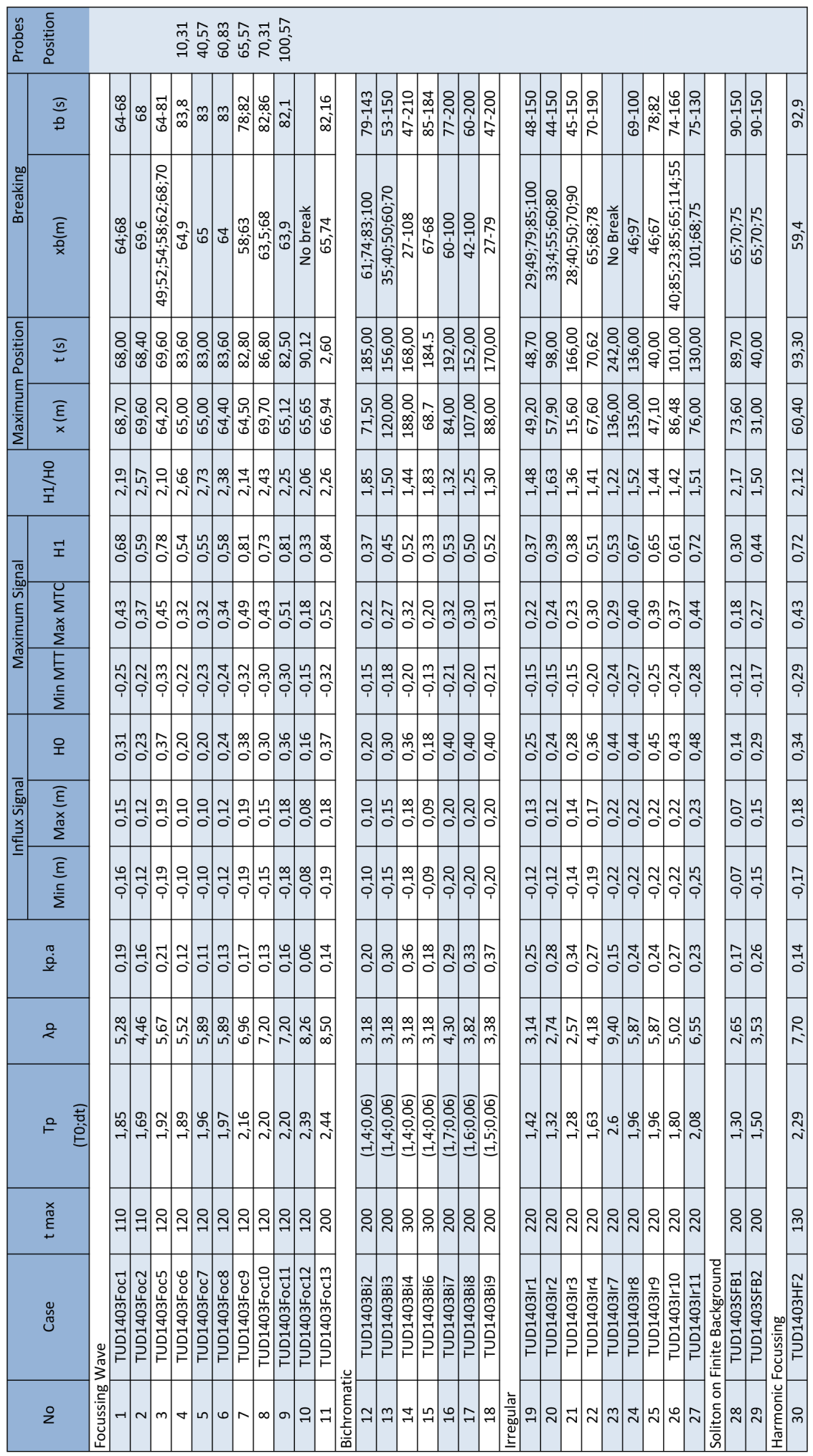




\section{A.2 Comparison of experiments and a-priori sim- ulations}

Bichromatic wave: TUD1403Bi3
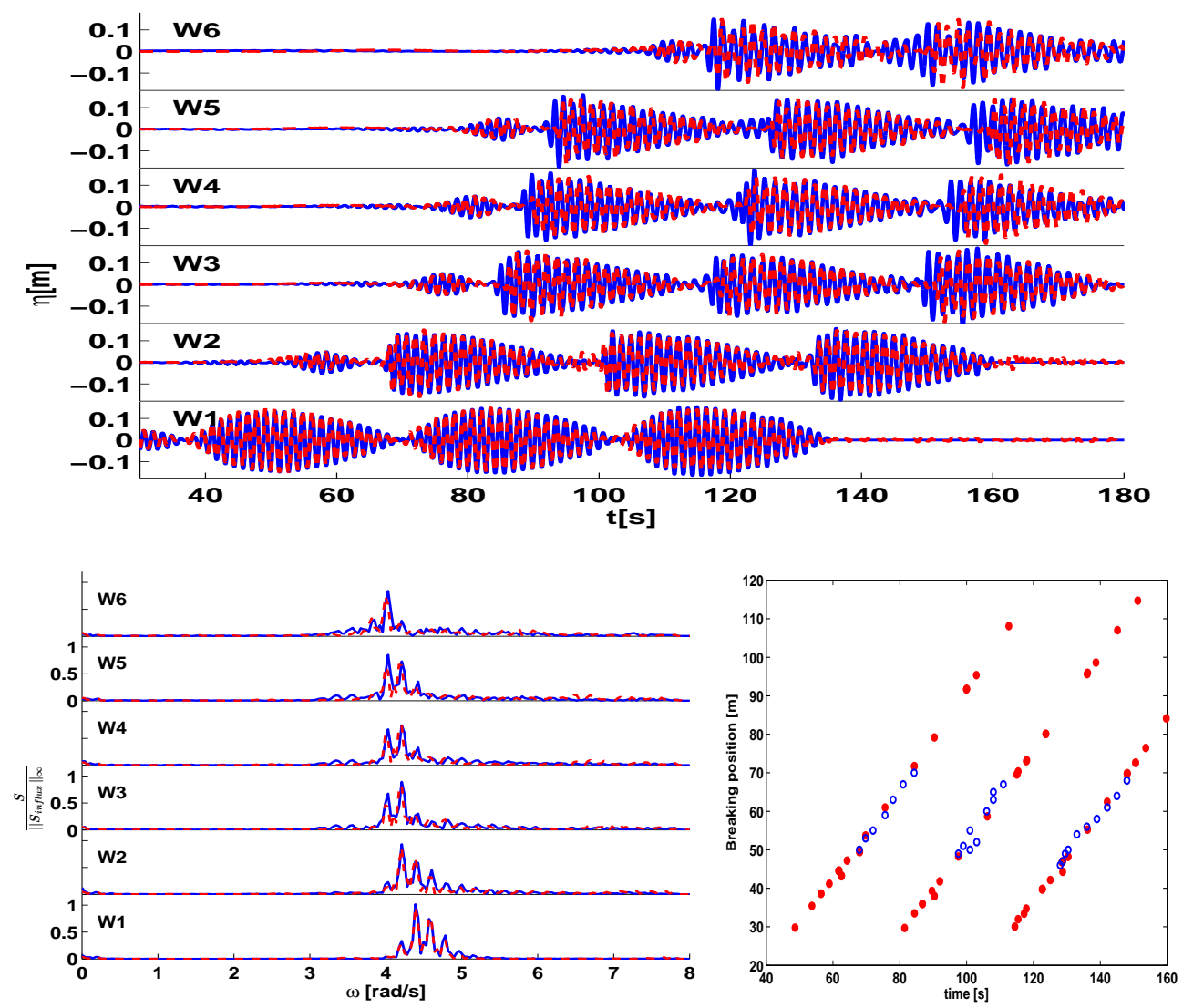

Figure A.1: Elevation time traces (top) and normalized amplitude spectra (left below) at positions W1, W2, W3, W4, W5, and W6 are shown for the measurement (blue, solid) and for the design simulation with model ABHS3 (red, dashed dot). At the right below, the positions of breaking of the design simulation (red, solid dots) and the limited observation $(x \in(50,70))$ in reality (in the movie, blue, open dots).

Table A.1: Wave properties: period, peak wavelength, steepness. Correlation at W1-W6 and relative computation time ( $\mathrm{Crel})$.

\begin{tabular}{|c|c|c|c|c|c|c|c|c|c|}
\hline$(\mathrm{T} 0, \mathrm{dt})$ & $\lambda_{p}$ & $k_{p} \cdot a$ & $\mathrm{~W} 1$ & $\mathrm{~W} 2$ & $\mathrm{~W} 3$ & $\mathrm{~W} 4$ & $\mathrm{~W} 5$ & W6 & Crel \\
\hline$(1.4,0.06)$ & 3.18 & 0.3 & 0.97 & 0.86 & 0.75 & 0.68 & 0.67 & 0.63 & 1.83 \\
\hline
\end{tabular}


Irregular wave: TUD1403Ir10
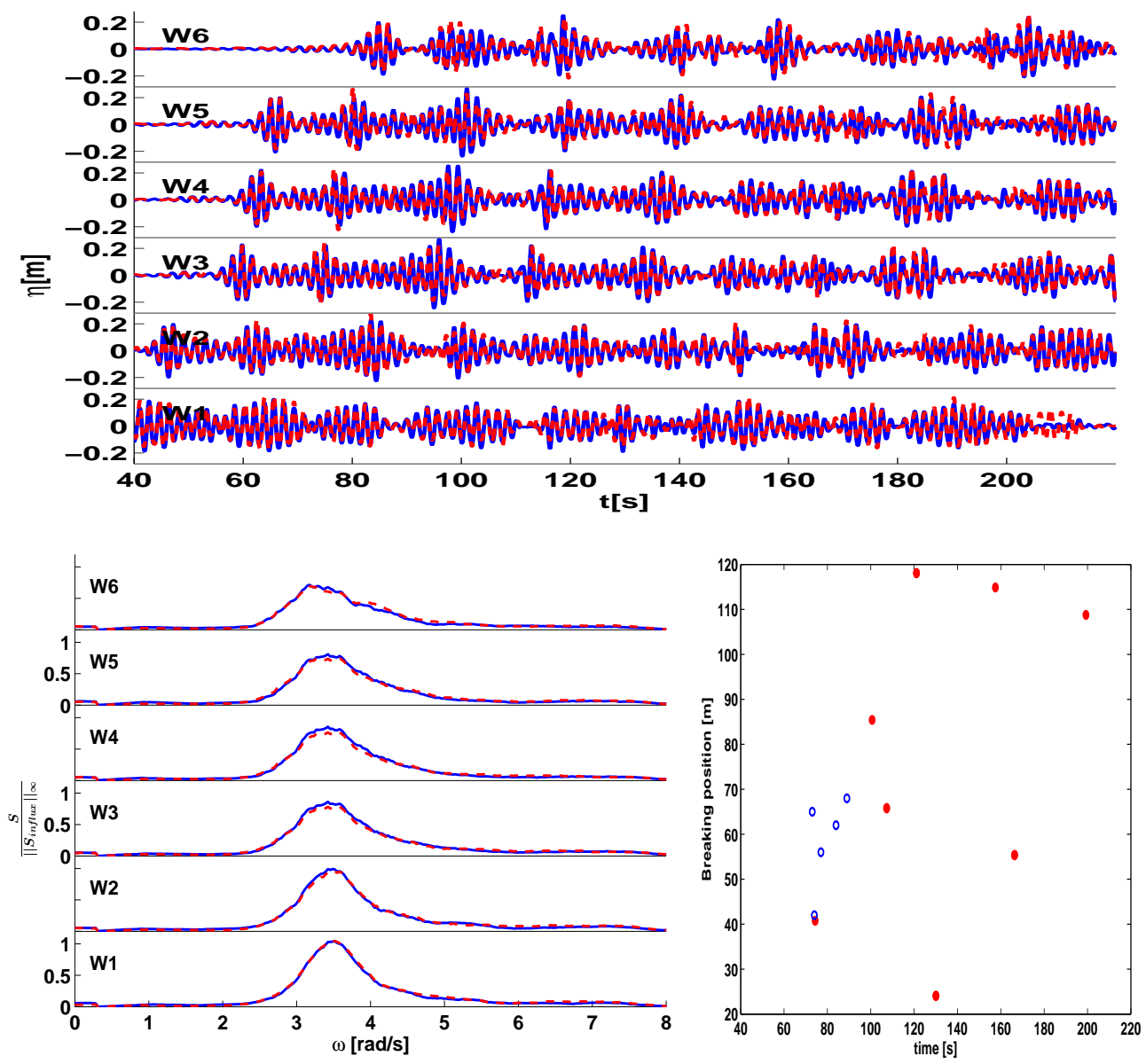

Figure A.2: Same as Fig. A.1 Now for TUD1403Ir10.

Table A.2: Same as Table A.1. Now for TUD1403Ir10.

\begin{tabular}{|c|c|c|c|c|c|c|c|c|c|}
\hline $\mathrm{Tp}$ & $\lambda_{p}$ & $k_{p} \cdot a$ & $\mathrm{~W} 1$ & $\mathrm{~W} 2$ & $\mathrm{~W} 3$ & $\mathrm{~W} 4$ & $\mathrm{~W} 5$ & $\mathrm{~W} 6$ & Crel \\
\hline 1.96 & 5.87 & 0.24 & 0.94 & 0.92 & 0.85 & 0.84 & 0.86 & 0.81 & 1.87 \\
\hline
\end{tabular}


Focussing wave: TUD1403Foc7
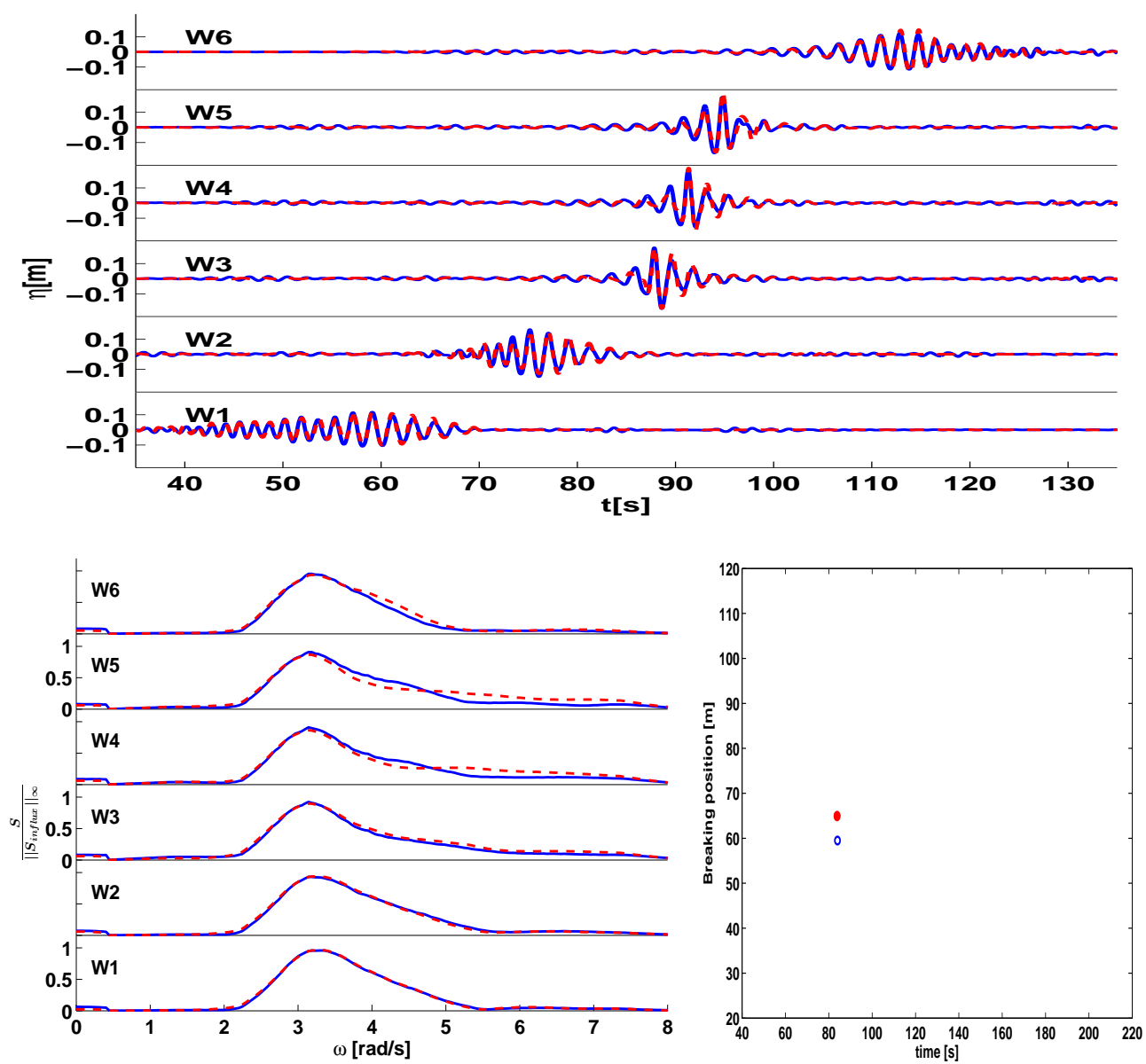

Figure A.3: Same as Fig. A.1, Now for TUD1403Foc'7 case.

Table A.3: Same as Table A.1. Now for TUD1403Foc'7 case.

\begin{tabular}{|c|c|c|c|c|c|c|c|c|c|}
\hline $\mathrm{Tp}$ & $\lambda_{p}$ & $k_{p} . a$ & $\mathrm{~W} 1$ & $\mathrm{~W} 2$ & $\mathrm{~W} 3$ & $\mathrm{~W} 4$ & $\mathrm{~W} 5$ & $\mathrm{~W} 6$ & Crel \\
\hline 1.96 & 5.89 & 0.11 & 0.93 & 0.91 & 0.88 & 0.87 & 0.88 & 0.92 & 1.5 \\
\hline
\end{tabular}




\section{A.3 Comparison of experiments and a-posteriori simulations}

Focussing wave group: TUD1403Foc1
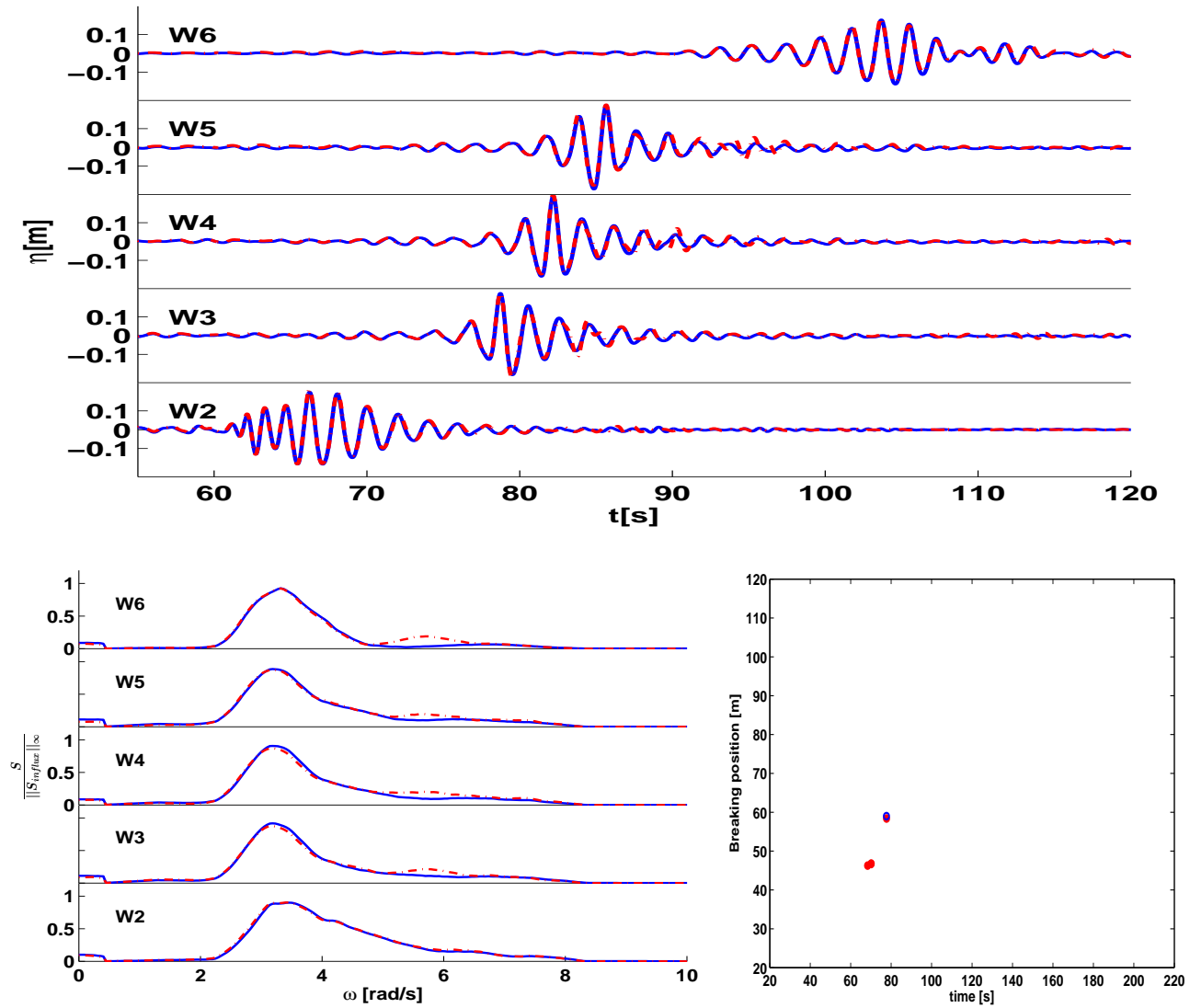

Figure A.4: Elevation time traces (top) and normalized amplitude spectra (left below) at positions W2, W3, W4, W5, and W6 are shown for the measurement (blue, solid) and for the a-posteriori simulation with model ABHS3 (red, dashed dot). At the right below, the positions of breaking of the design simulation (red, solid dots) and the limited observation $(x \in(50,70))$ in reality (in the movie, blue, open dots).

Table A.4: Wave properties: peak period, peak wavelength, steepness. Correlation at W2-W6 and relative computation time (Crel).

\begin{tabular}{|c|c|c|c|c|c|c|c|c|}
\hline $\mathrm{Tp}$ & $\lambda_{p}$ & $k_{p} \cdot a$ & $\mathrm{~W} 2$ & $\mathrm{~W} 3$ & $\mathrm{~W} 4$ & $\mathrm{~W} 5$ & $\mathrm{~W} 6$ & Crel \\
\hline 1.85 & 5.3 & 0.19 & 0.98 & 0.97 & 0.96 & 0.96 & 0.97 & 1.35 \\
\hline
\end{tabular}


Focussing wave group: TUD1403Foc2
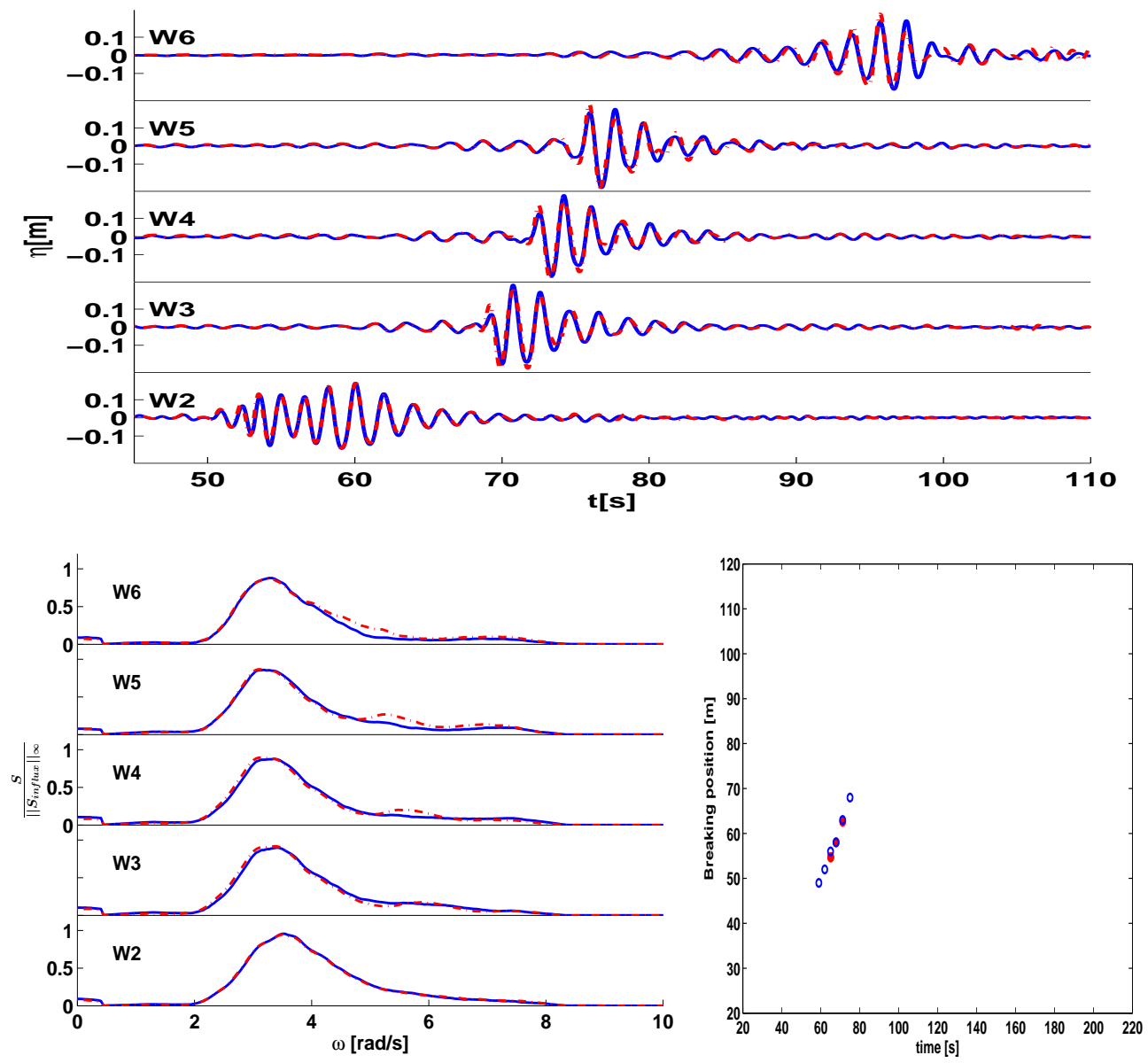

Figure A.5: Same as Fig. A.4, now for TUD1403Foc2 case.

Table A.5: Same as Table A.4 Now for TUD1403Foc2 case.

\begin{tabular}{|c|c|c|c|c|c|c|c|c|}
\hline $\mathrm{Tp}$ & $\lambda_{p}$ & $k_{p} \cdot a$ & $\mathrm{~W} 2$ & $\mathrm{~W} 3$ & $\mathrm{~W} 4$ & W5 & W6 & Crel \\
\hline 1.7 & 4.46 & 0.16 & 0.98 & 0.94 & 0.94 & 0.94 & 0.88 & 0.68 \\
\hline
\end{tabular}


Focussing wave group: TUD1403Foc5
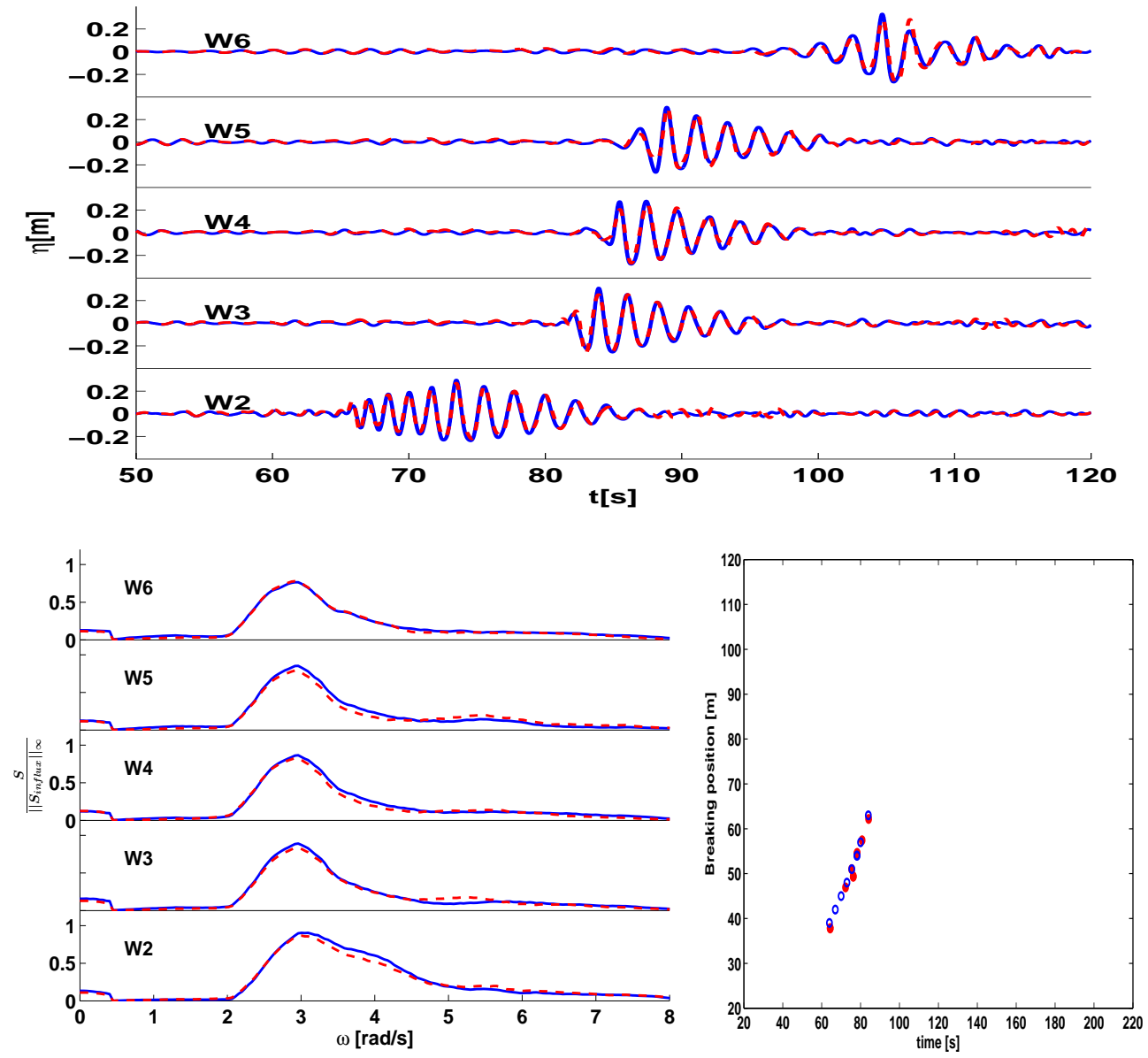

Figure A.6: Same as Fig. A.4, now for TUD1403Foc5 case.

Table A.6: Same as Table A.4 Now for TUD1403Foc5 case.

\begin{tabular}{|c|c|c|c|c|c|c|c|c|}
\hline $\mathrm{Tp}$ & $\lambda_{p}$ & $k_{p} \cdot a$ & $\mathrm{~W} 2$ & $\mathrm{~W} 3$ & $\mathrm{~W} 4$ & $\mathrm{~W} 5$ & W6 & Crel \\
\hline 1.92 & 5.7 & 0.21 & 0.96 & 0.96 & 0.96 & 0.94 & 0.93 & 0.92 \\
\hline
\end{tabular}


Focussing wave group: TUD1403Foc6
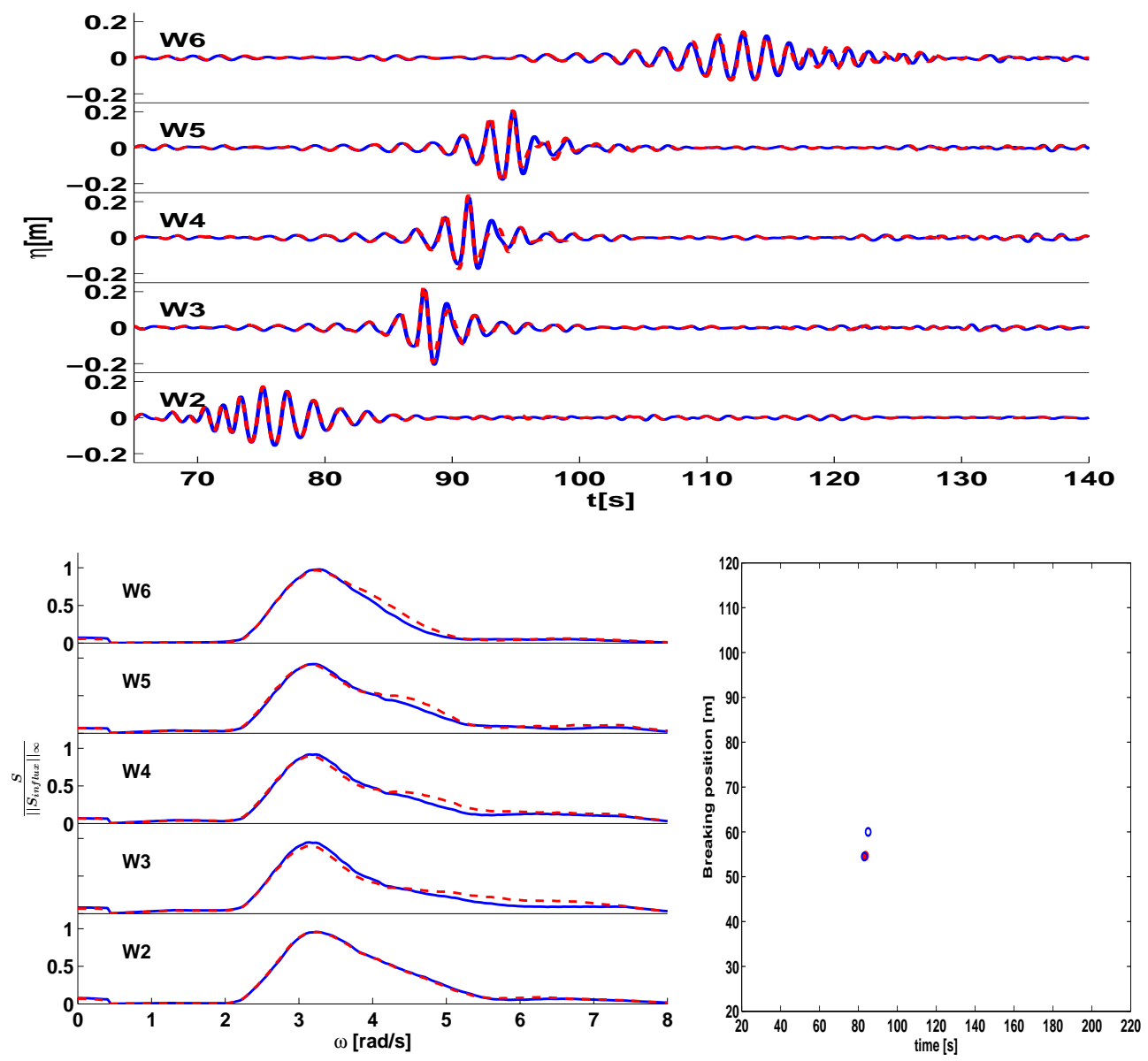

Figure A.7: Same as Fig. A.4, now for TUD1403Foc6 case.

Table A.7: Same as Table A.4 Now for TUD1403Foc6 case.

\begin{tabular}{|c|c|c|c|c|c|c|c|c|}
\hline $\mathrm{Tp}$ & $\lambda_{p}$ & $k_{p} \cdot a$ & $\mathrm{~W} 2$ & $\mathrm{~W} 3$ & $\mathrm{~W} 4$ & $\mathrm{~W} 5$ & $\mathrm{~W} 6$ & Crel \\
\hline 1.89 & 5.52 & 0.12 & 0.99 & 0.96 & 0.95 & 0.96 & 0.95 & 1.06 \\
\hline
\end{tabular}


Focussing wave group: TUD1403Foc7
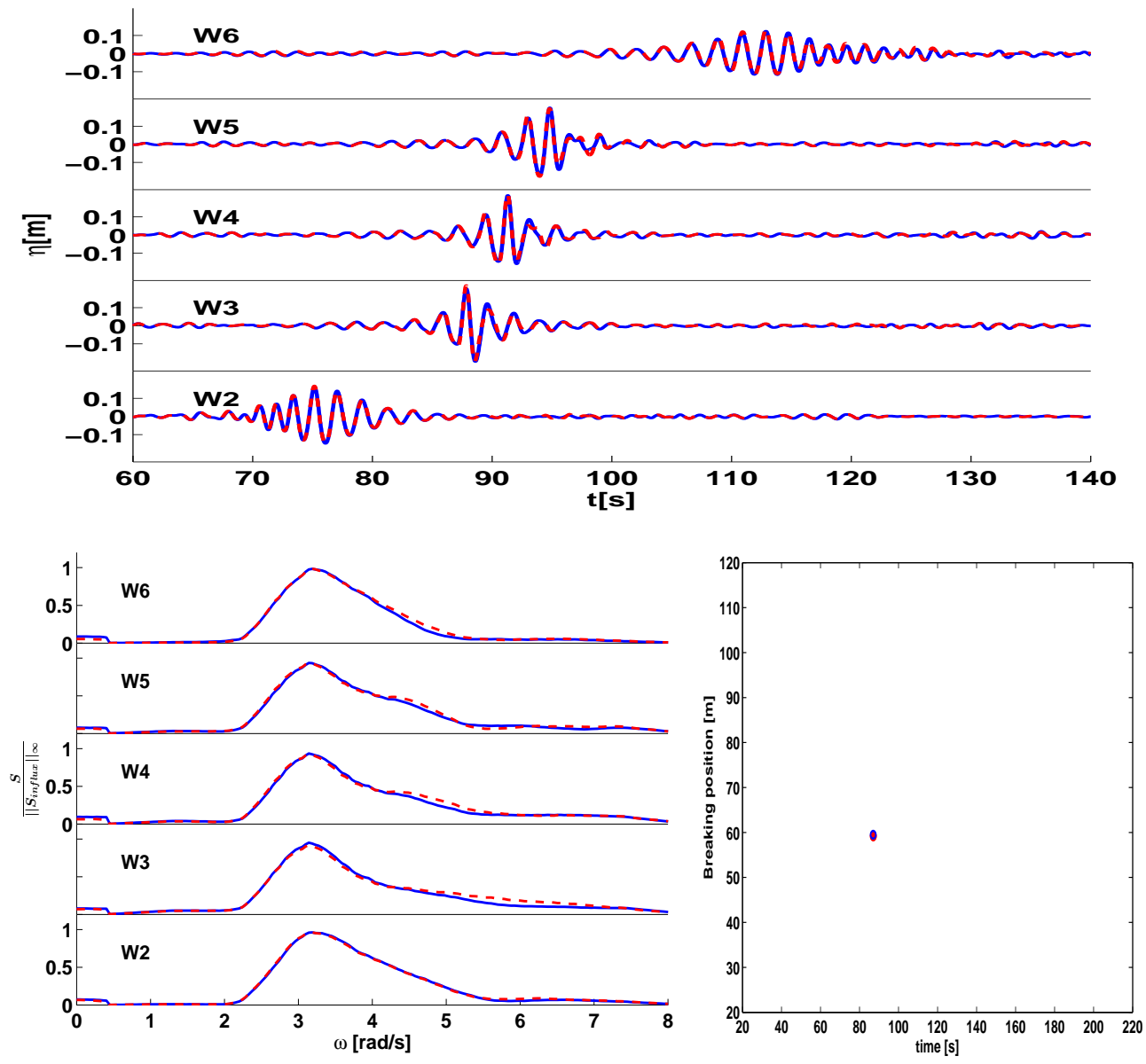

Figure A.8: Same as Fig. A.4, now for TUD1403Foc7 case.

Table A.8: Same as Table A.4 Now for TUD1403Foc'7 case.

\begin{tabular}{|c|c|c|c|c|c|c|c|c|}
\hline $\mathrm{Tp}$ & $\lambda_{p}$ & $k_{p} \cdot a$ & $\mathrm{~W} 2$ & $\mathrm{~W} 3$ & $\mathrm{~W} 4$ & $\mathrm{~W} 5$ & W6 & Crel \\
\hline 1.96 & 5.89 & 0.11 & 0.99 & 0.97 & 0.98 & 0.98 & 0.97 & 1.13 \\
\hline
\end{tabular}


Focussing wave group: TUD1403Foc8
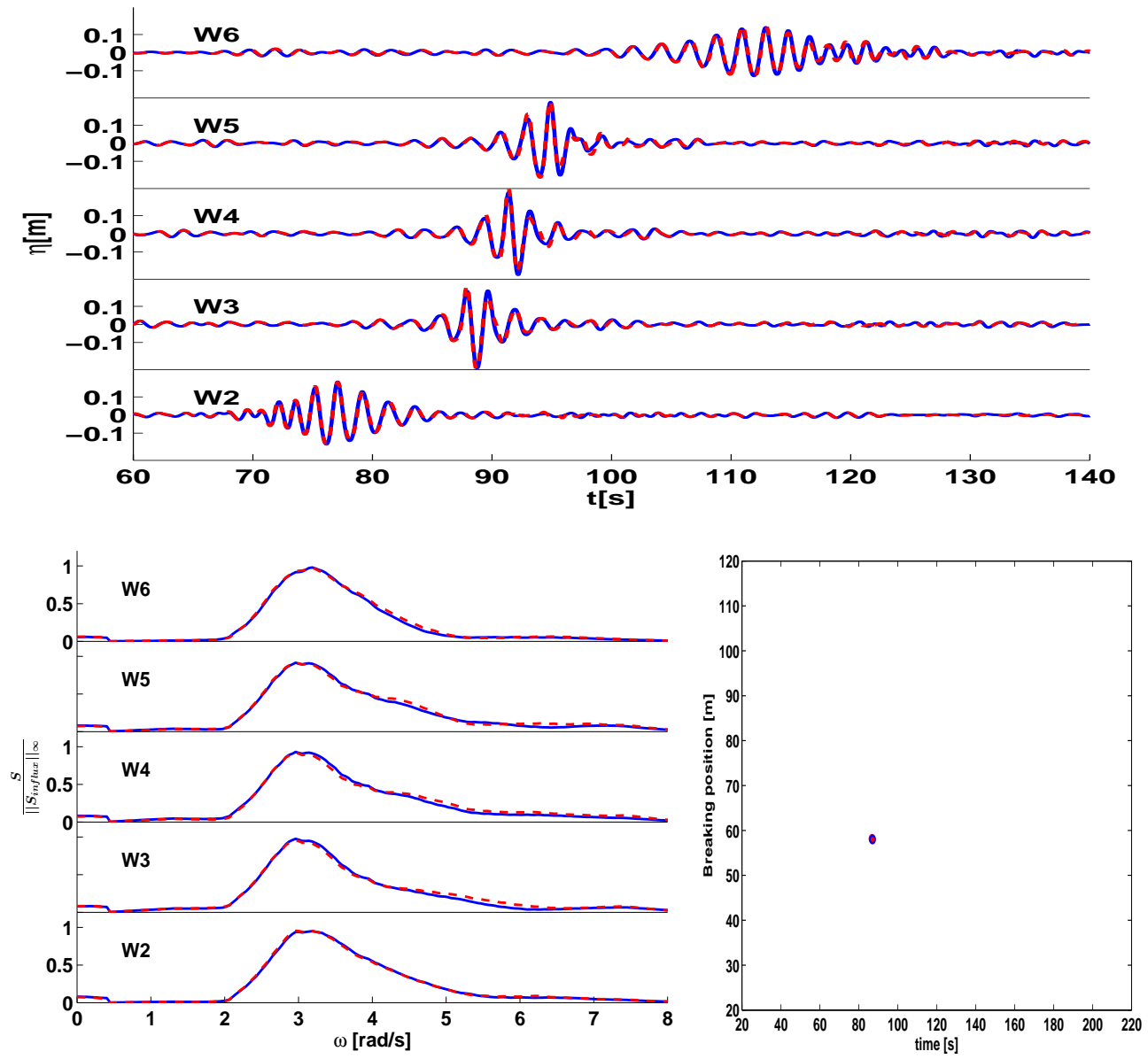

Figure A.9: Same as Fig. A.4, now for TUD1403Foc8 case.

Table A.9: Same as Table A.4 Now for TUD1403Foc8 case.

\begin{tabular}{|c|c|c|c|c|c|c|c|c|}
\hline $\mathrm{Tp}$ & $\lambda_{p}$ & $k_{p} \cdot a$ & $\mathrm{~W} 2$ & $\mathrm{~W} 3$ & $\mathrm{~W} 4$ & $\mathrm{~W} 5$ & $\mathrm{~W} 6$ & Crel \\
\hline 1.96 & 5.89 & 0.13 & 0.99 & 0.97 & 0.97 & 0.97 & 0.97 & 0.56 \\
\hline
\end{tabular}


Focussing wave group: TUD1403Foc9
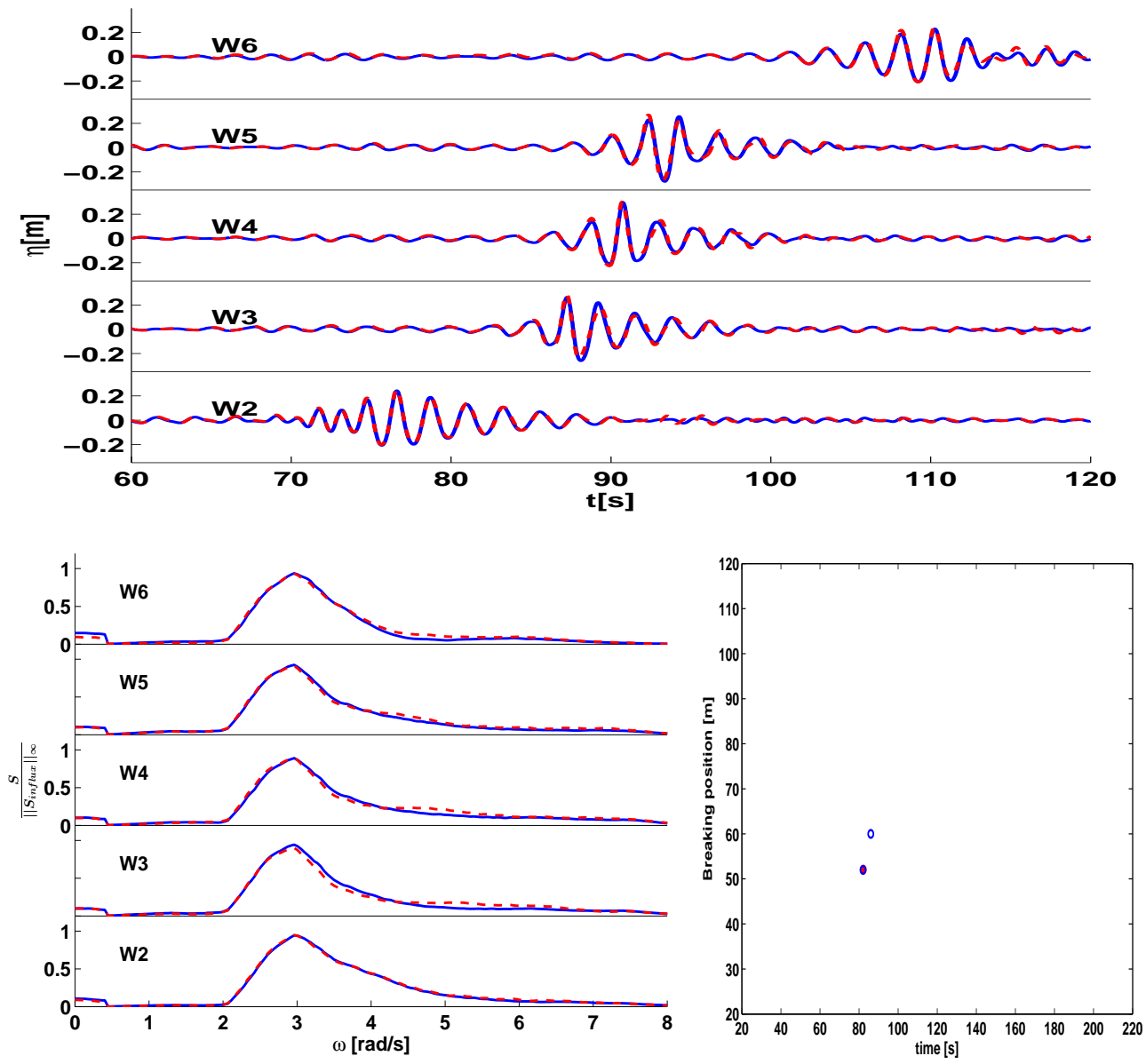

Figure A.10: Same as Fig. A.4 now for TUD1403Foc9 case.

Table A.10: Same as Table A.4. Now for TUD1403Foc9 case.

\begin{tabular}{|c|c|c|c|c|c|c|c|c|}
\hline $\mathrm{Tp}$ & $\lambda_{p}$ & $k_{p} \cdot a$ & $\mathrm{~W} 2$ & $\mathrm{~W} 3$ & $\mathrm{~W} 4$ & W5 & W6 & Crel \\
\hline 2.16 & 6.96 & 0.17 & 0.99 & 0.96 & 0.96 & 0.96 & 0.96 & 1.17 \\
\hline
\end{tabular}


Focussing wave group: TUD1403Foc10
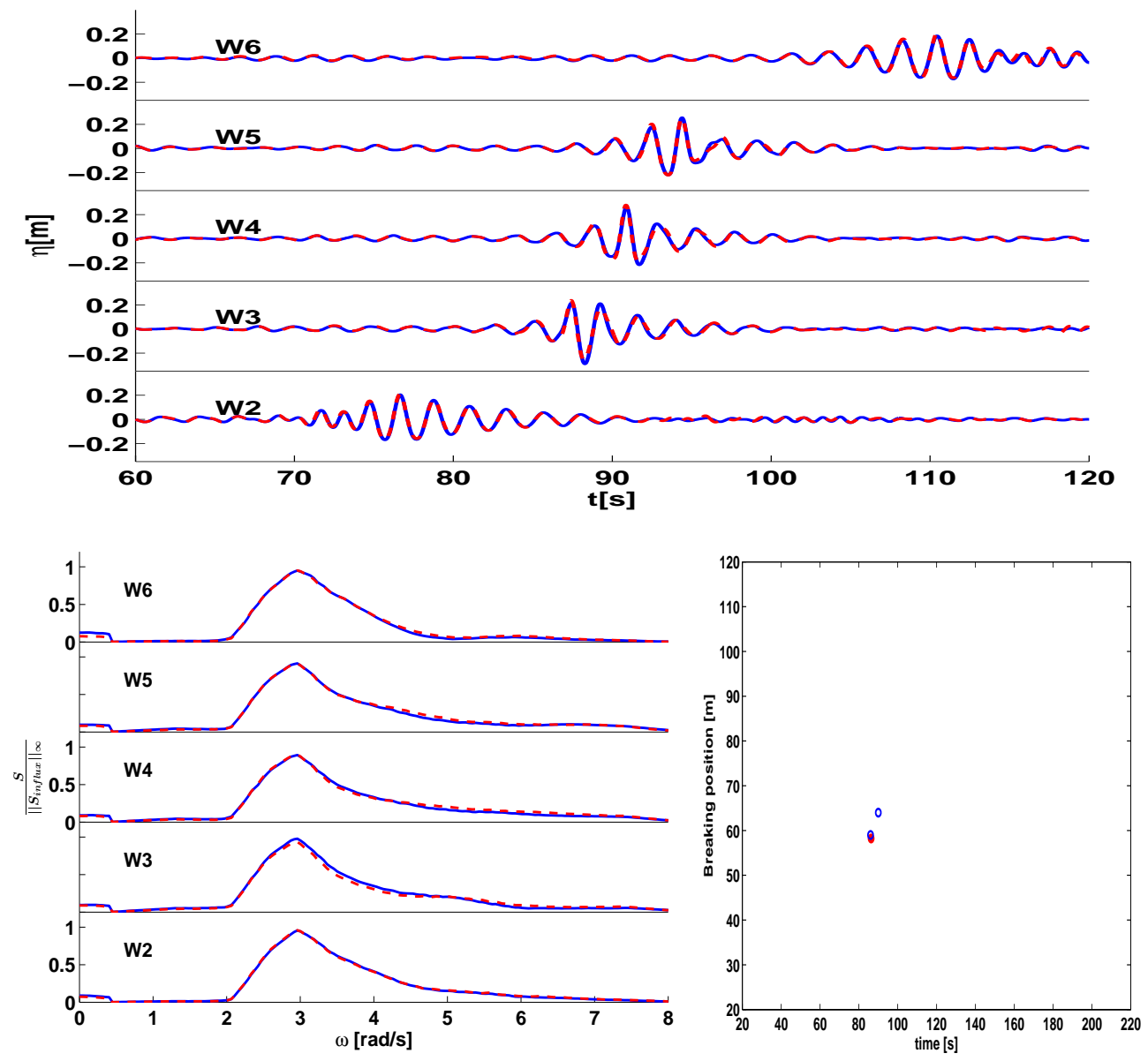

Figure A.11: Same as Fig. A.4, now for TUD1403Foc10 case.

Table A.11: Same as Table A.4. Now for TUD1403Foc10 case.

\begin{tabular}{|c|c|c|c|c|c|c|c|c|}
\hline $\mathrm{Tp}$ & $\lambda_{p}$ & $k_{p} \cdot a$ & $\mathrm{~W} 2$ & $\mathrm{~W} 3$ & $\mathrm{~W} 4$ & $\mathrm{~W} 5$ & W6 & Crel \\
\hline 2.2 & 7.2 & 0.13 & 0.99 & 0.97 & 0.97 & 0.98 & 0.97 & 1.08 \\
\hline
\end{tabular}


Focussing wave group: TUD1403Foc11
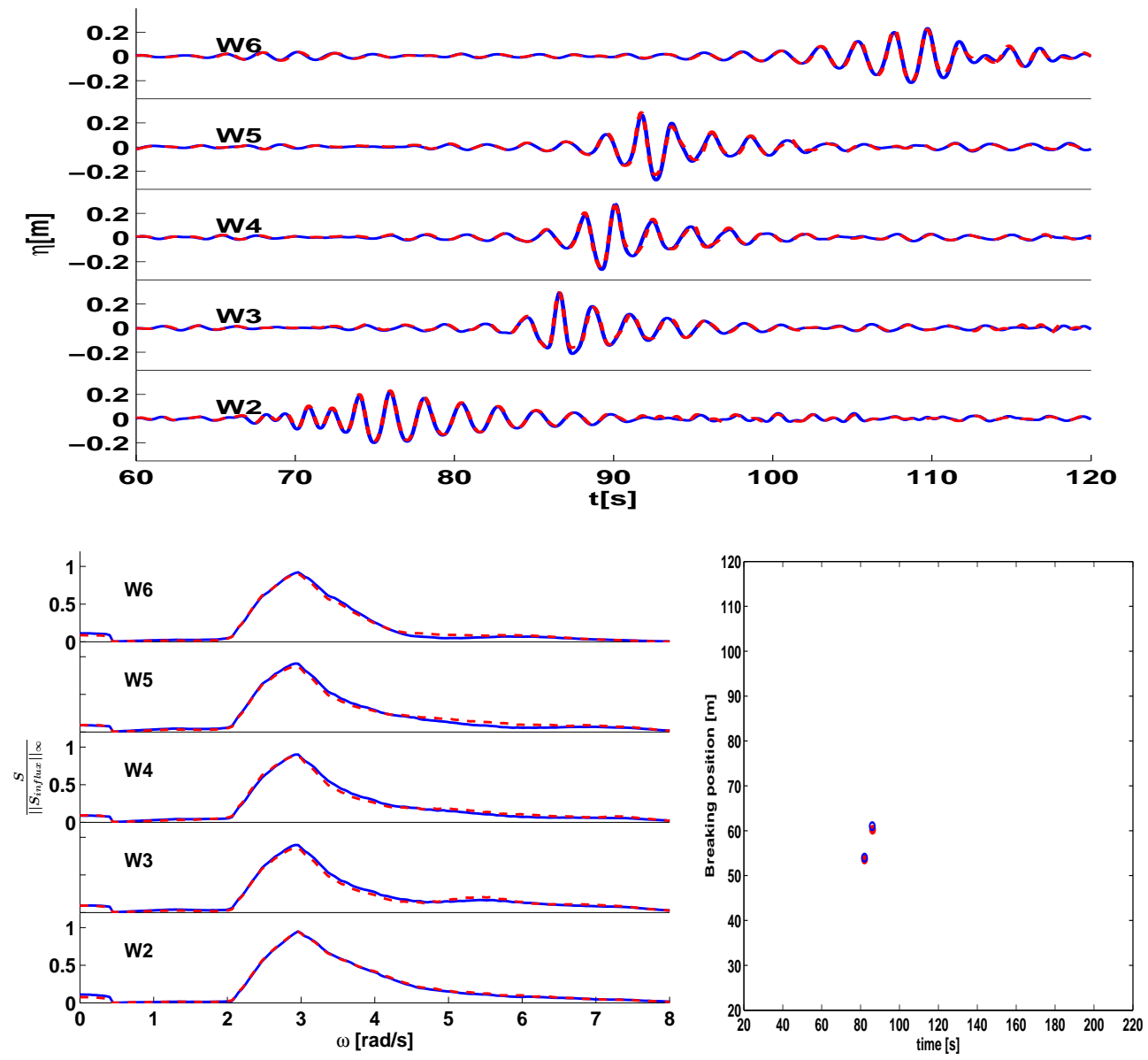

Figure A.12: Same as Fig. A.4 now for TUD1403Foc11 case.

Table A.12: Same as Table A.4 Now for TUD1403Foc11 case.

\begin{tabular}{|c|c|c|c|c|c|c|c|c|}
\hline $\mathrm{Tp}$ & $\lambda_{p}$ & $k_{p} \cdot a$ & $\mathrm{~W} 2$ & $\mathrm{~W} 3$ & $\mathrm{~W} 4$ & $\mathrm{~W} 5$ & W6 & Crel \\
\hline 2.2 & 7.2 & 0.16 & 0.99 & 0.97 & 0.98 & 0.98 & 0.98 & 1.21 \\
\hline
\end{tabular}


Focussing wave group: TUD1403Foc12 (non breaking)
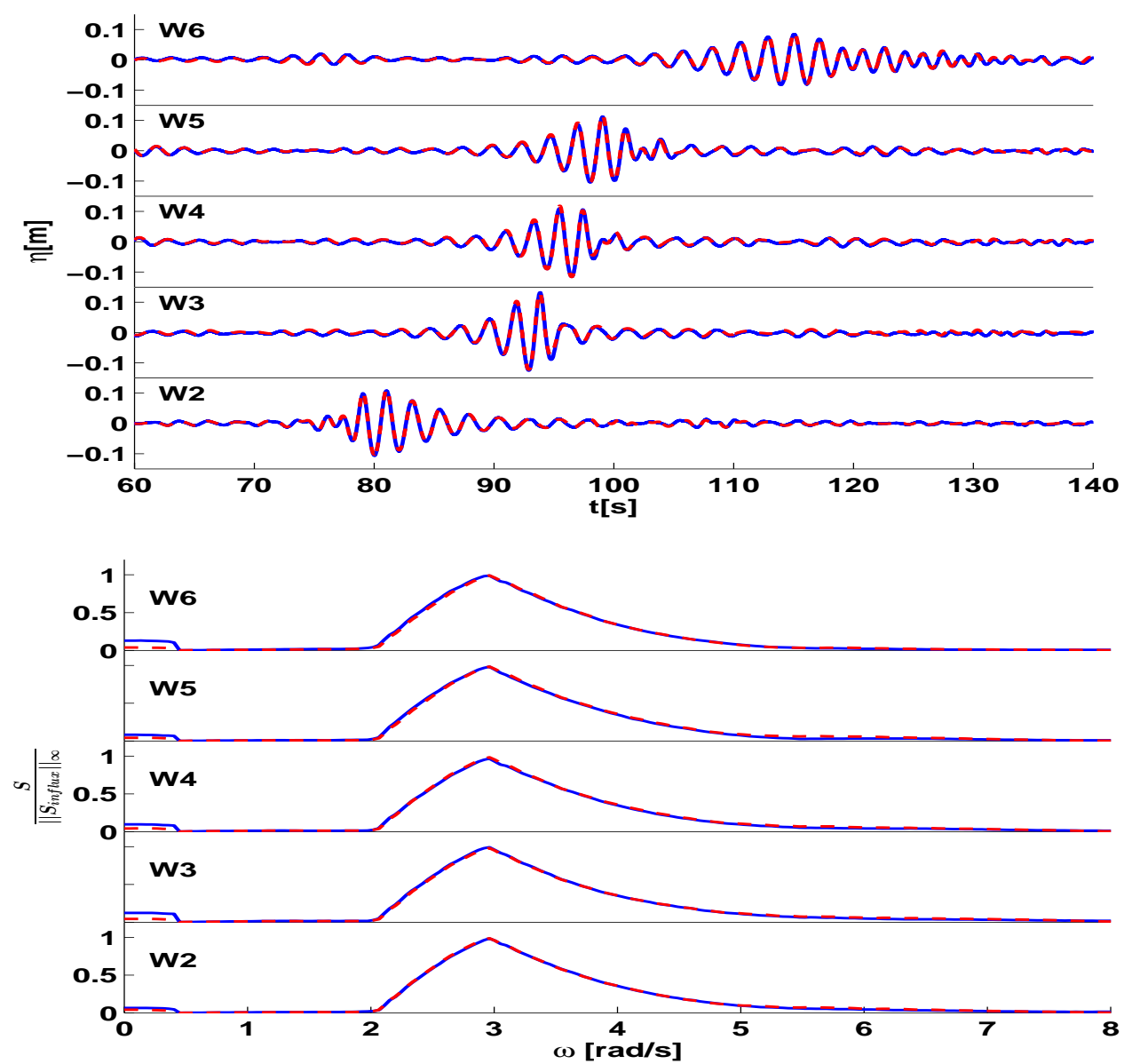

Figure A.13: Same as Fig. A.4, now for TUD1403Foc12 case.

Table A.13: Same as Table A.4. Now for TUD1403Foc12 case.

\begin{tabular}{|c|c|c|c|c|c|c|c|c|}
\hline $\mathrm{Tp}$ & $\lambda_{p}$ & $k_{p} \cdot a$ & $\mathrm{~W} 2$ & $\mathrm{~W} 3$ & $\mathrm{~W} 4$ & $\mathrm{~W} 5$ & $\mathrm{~W} 6$ & Crel \\
\hline 2.38 & 8.26 & 0.06 & 0.97 & 0.96 & 0.97 & 0.97 & 0.97 & 1.70 \\
\hline
\end{tabular}


Focussing wave group: TUD1403Foc13
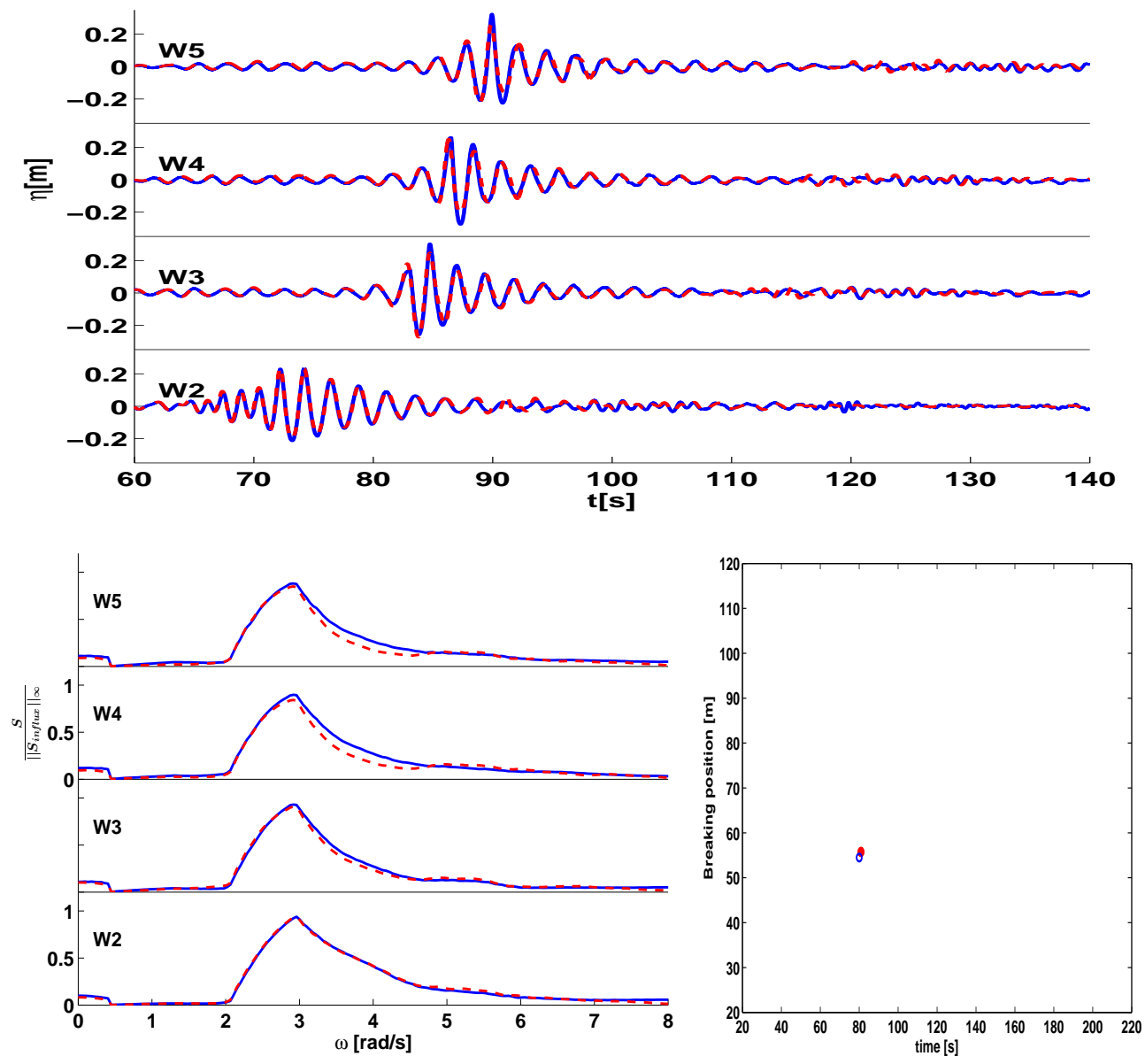

Figure A.14: Same as Fig. A.4 now for TUD1403Foc13 case.

Table A.14: Same as Table A.4 Now for TUD1403Foc13 case.

\begin{tabular}{|c|c|c|c|c|c|c|c|}
\hline $\mathrm{Tp}$ & $\lambda_{p}$ & $k_{p} \cdot a$ & $\mathrm{~W} 2$ & $\mathrm{~W} 3$ & $\mathrm{~W} 4$ & W5 & Crel \\
\hline 2.4 & 8.25 & 0.14 & 0.98 & 0.96 & 0.95 & 0.96 & 2.44 \\
\hline
\end{tabular}


Bichromatic wave: TUD1403Bi2
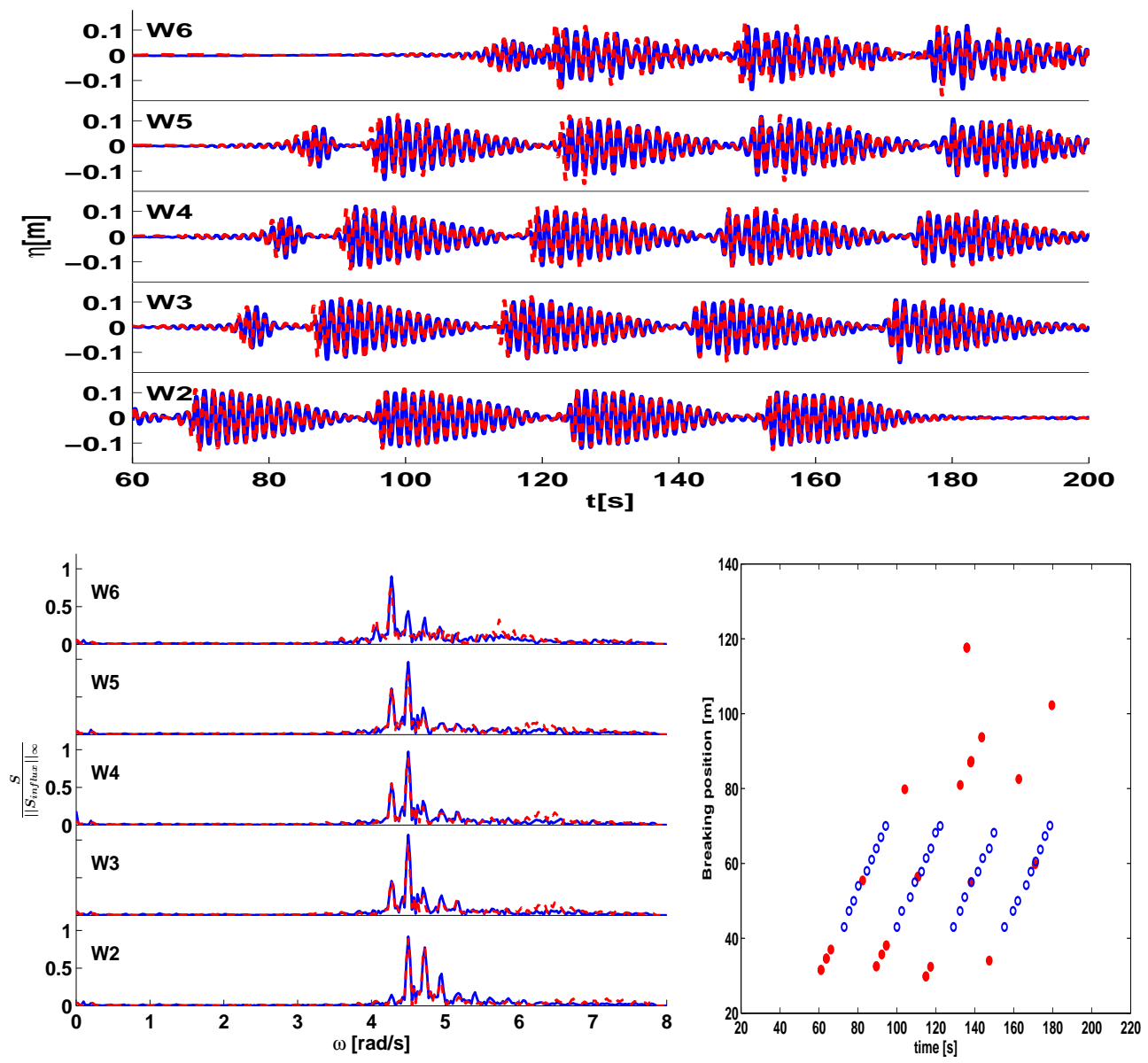

Figure A.15: Same as Fig. A.4 now for TUD1403Bi2 case.

Table A.15: Same as Table A.4 Now for TUD1403Bi2 case.

\begin{tabular}{|c|c|c|c|c|c|c|c|c|}
\hline (T0,dt) & $\lambda_{p}$ & $k_{p} . a$ & W2 & W3 & W4 & W5 & W6 & Crel \\
\hline$(1.4,0.06)$ & 3.18 & 0.20 & 0.93 & 0.88 & 0.86 & 0.83 & 0.67 & 2.3 \\
\hline
\end{tabular}


Bichromatic wave: TUD1403Bi3
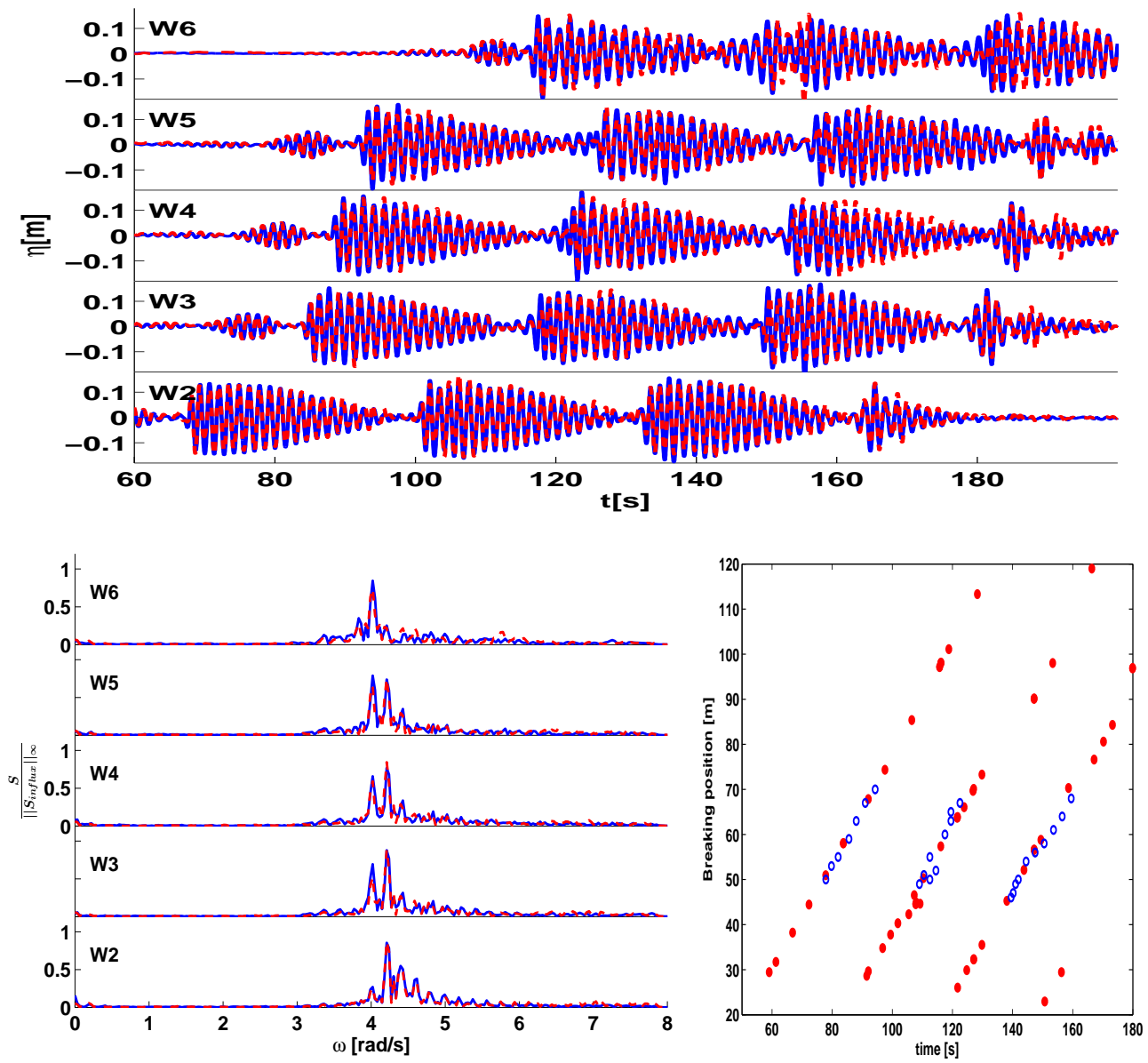

Figure A.16: Same as Fig. A.4 now for TUD1403Bi3 case.

Table A.16: Same as Table A.4 Now for TUD1403Bi3 case.

\begin{tabular}{|c|c|c|c|c|c|c|c|c|}
\hline (T0,dt) & $\lambda_{p}$ & $k_{p} \cdot a$ & W2 & W3 & W4 & W5 & W6 & Crel \\
\hline$(1.4,0.06)$ & 3.18 & 0.3 & 0.96 & 0.90 & 0.87 & 0.84 & 0.78 & 3.2 \\
\hline
\end{tabular}


Bichromatic wave: TUD1403Bi4
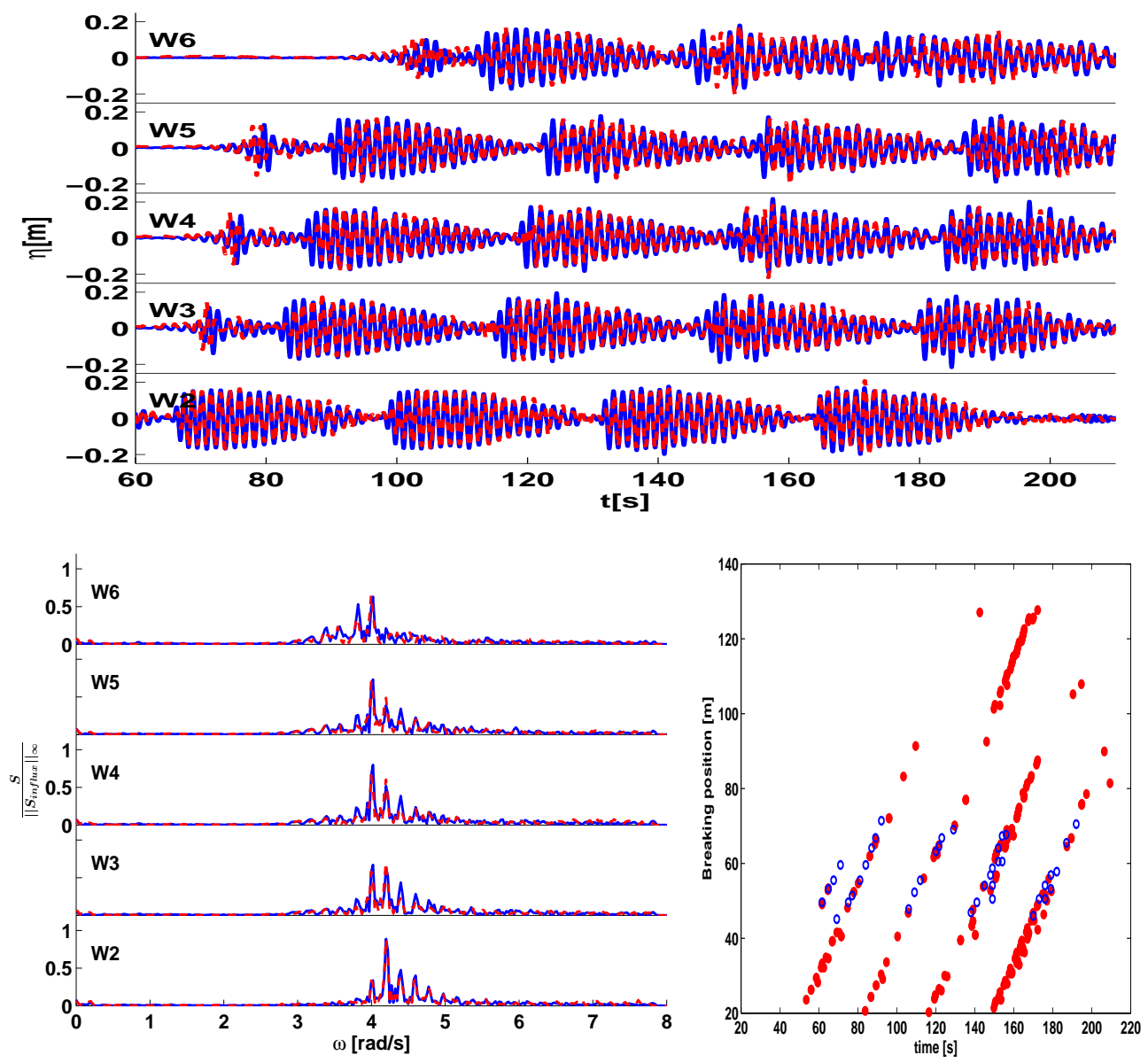

Figure A.17: Same as Fig. A.4 now for TUD1403Bi4 case

Table A.17: Same as Table A.4 Now for TUD1403Bi4 case.

\begin{tabular}{|c|c|c|c|c|c|c|c|c|}
\hline (T0,dt) & $\lambda_{p}$ & $k_{p} . a$ & W2 & W3 & W4 & W5 & W6 & Crel \\
\hline$(1.4,0.06)$ & 3.18 & 0.36 & 0.93 & 0.82 & 0.79 & 0.75 & 0.63 & 3.6 \\
\hline
\end{tabular}


Bichromatic wave: TUD1403Bi6
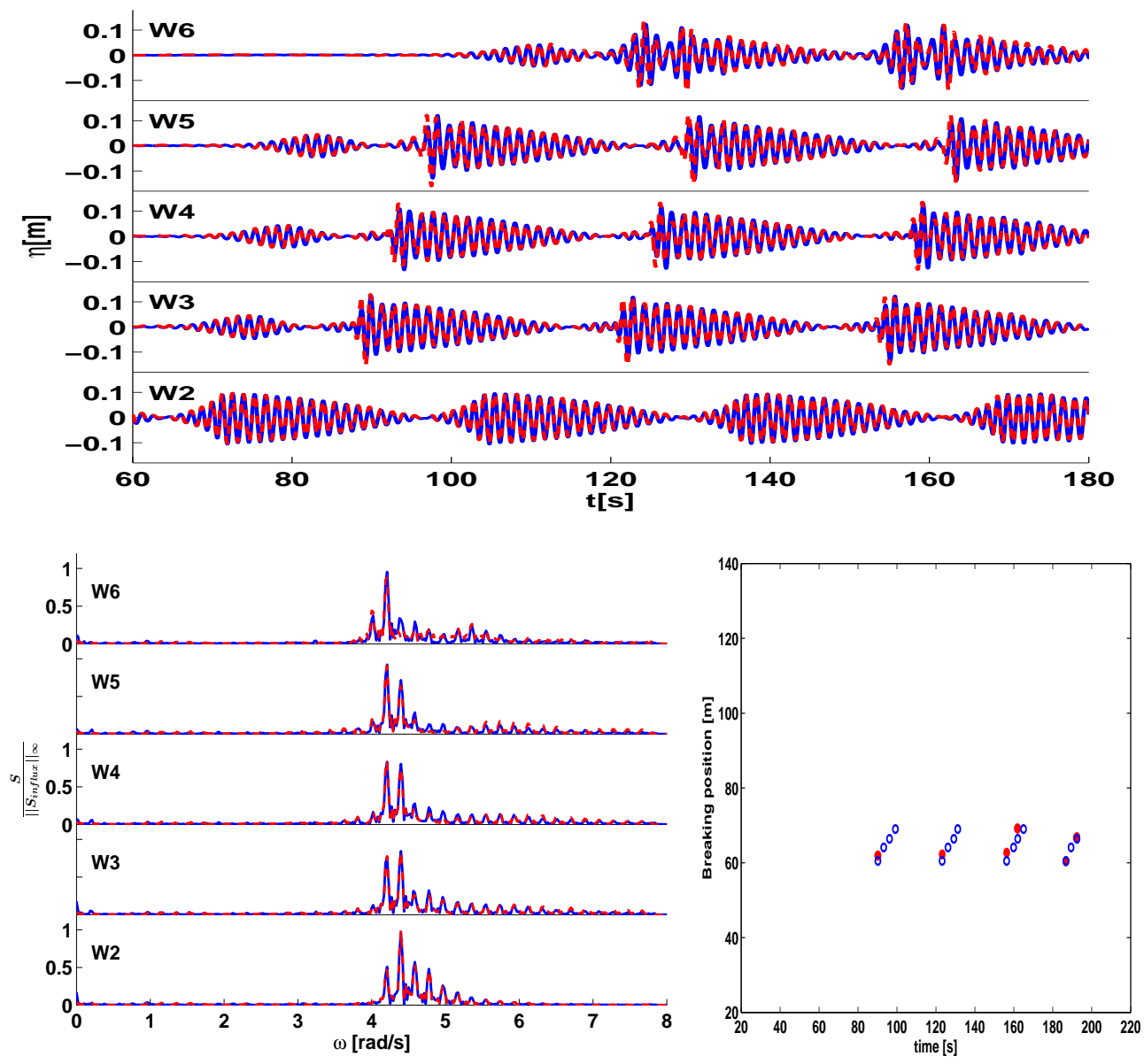

Figure A.18: Same as Fig. A.4 now for TUD1403Bi6 case

Table A.18: Same as Table A.4 Now for TUD1403Bi6 case.

\begin{tabular}{|c|c|c|c|c|c|c|c|c|}
\hline$(\mathrm{T} 0, \mathrm{dt})$ & $\lambda_{p}$ & $k_{p} \cdot a$ & $\mathrm{~W} 2$ & $\mathrm{~W} 3$ & $\mathrm{~W} 4$ & $\mathrm{~W} 5$ & W6 & Crel \\
\hline$(1.4,0.06)$ & 3.18 & 0.18 & 0.98 & 0.94 & 0.92 & 0.90 & 0.86 & 1.9 \\
\hline
\end{tabular}


Bichromatic wave: TUD1403Bi7
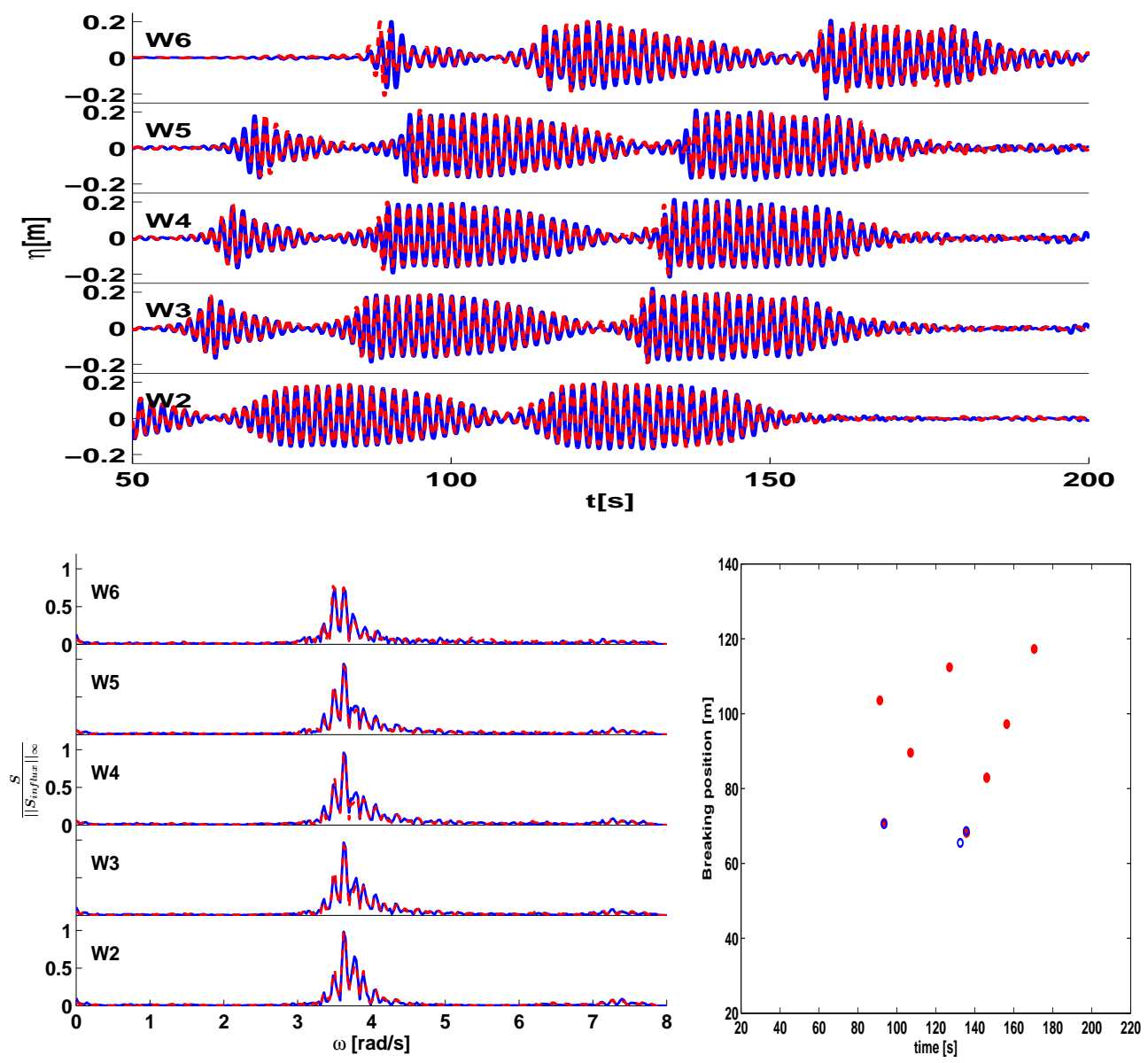

Figure A.19: Same as Fig. A.4 now for TUD1403Bit case

Table A.19: Same as Table A.4 Now for TUD1403Bi7 case.

\begin{tabular}{|c|c|c|c|c|c|c|c|c|}
\hline$(\mathrm{T} 0, \mathrm{dt})$ & $\lambda_{p}$ & $k_{p} \cdot a$ & $\mathrm{~W} 2$ & $\mathrm{~W} 3$ & $\mathrm{~W} 4$ & $\mathrm{~W} 5$ & W6 & Crel \\
\hline$(1.7,0.06)$ & 4.3 & 0.29 & 0.98 & 0.97 & 0.96 & 0.95 & 0.92 & 1.4 \\
\hline
\end{tabular}


Bichromatic wave: TUD1403Bi8
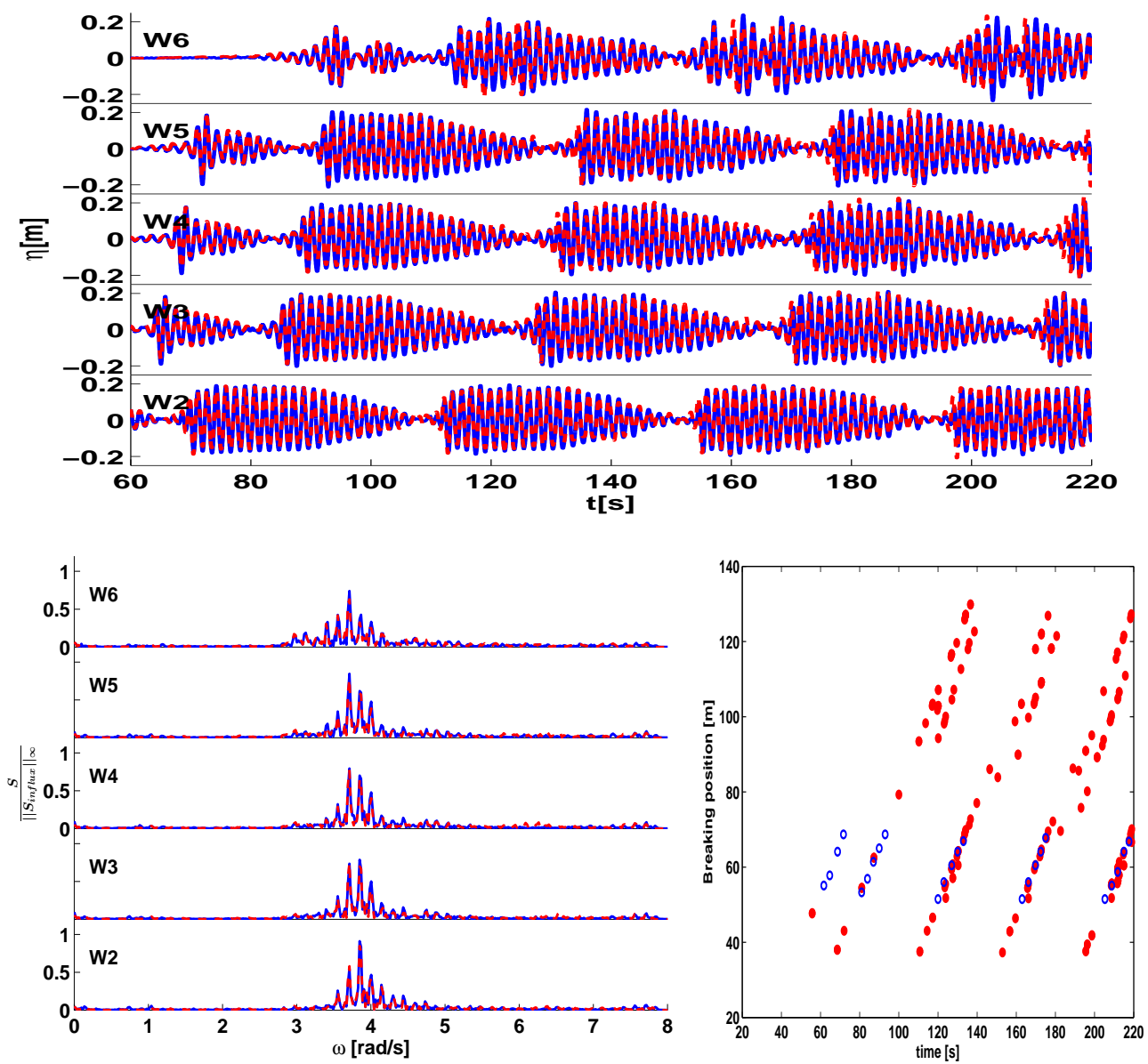

Figure A.20: Same as Fig. A.4 now for TUD1403Bi8 case.

Table A.20: Same as Table A.4 Now for TUD1403Bi8 case.

\begin{tabular}{|c|c|c|c|c|c|c|c|c|}
\hline (T0,dt) & $\lambda_{p}$ & $k_{p} \cdot a$ & W2 & W3 & W4 & W5 & W6 & Crel \\
\hline$(1.6,0.06)$ & 3.82 & 0.33 & 0.96 & 0.93 & 0.93 & 0.91 & 0.81 & 2.3 \\
\hline
\end{tabular}


Bichromatic wave: TUD1403Bi9
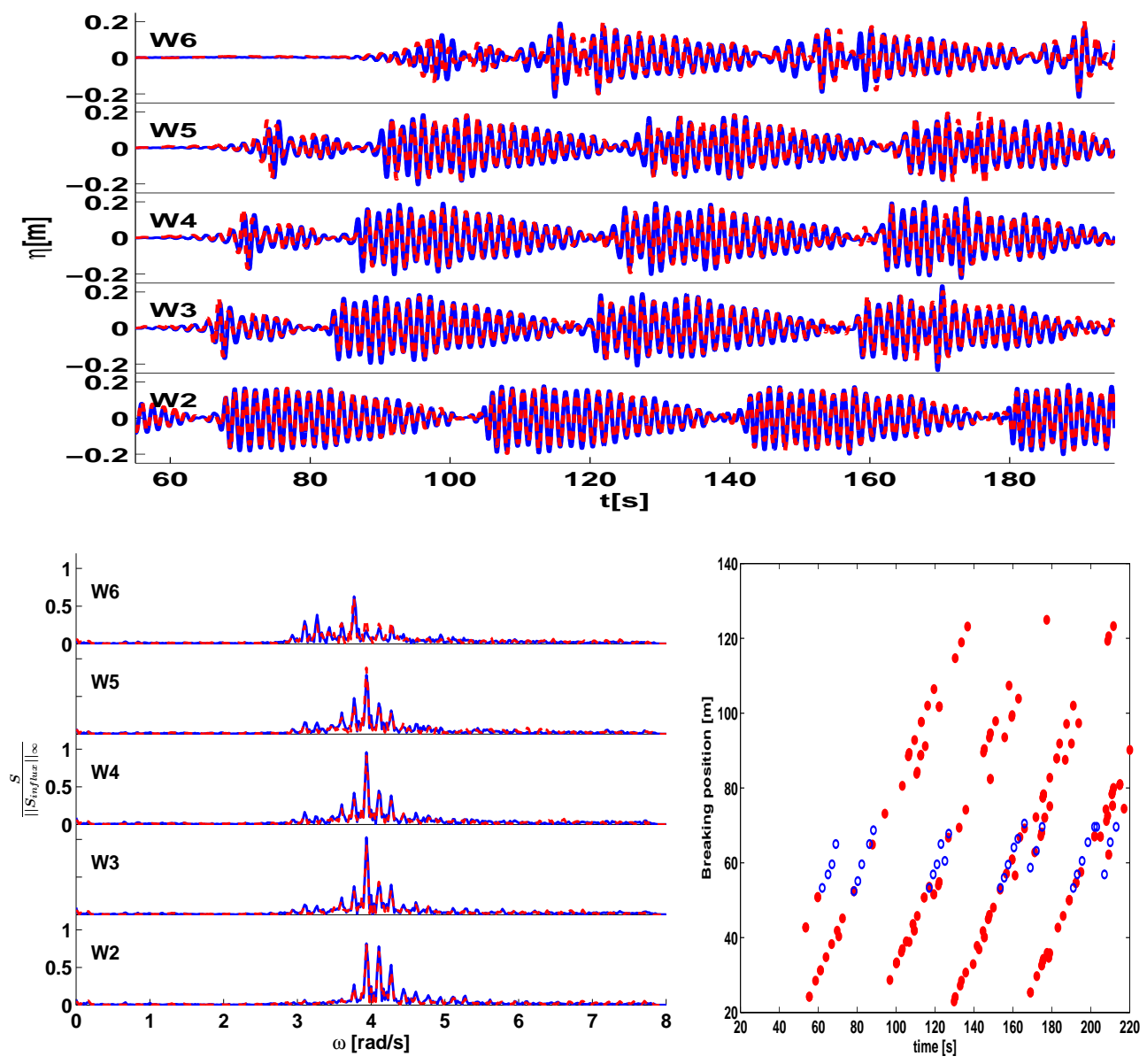

Figure A.21: Same as Fig. A.4 now for TUD1403Bi9 case.

Table A.21: Same as Table A.4 Now for TUD1403Bi9 case.

\begin{tabular}{|c|c|c|c|c|c|c|c|c|}
\hline (T0,dt) & $\lambda_{p}$ & $k_{p} . a$ & W2 & W3 & W4 & W5 & W6 & Crel \\
\hline$(1.5,0.06)$ & 3.38 & 0.37 & 0.95 & 0.93 & 0.91 & 0.88 & 0.79 & 3.7 \\
\hline
\end{tabular}


Irregular wave: TUD1403Ir1
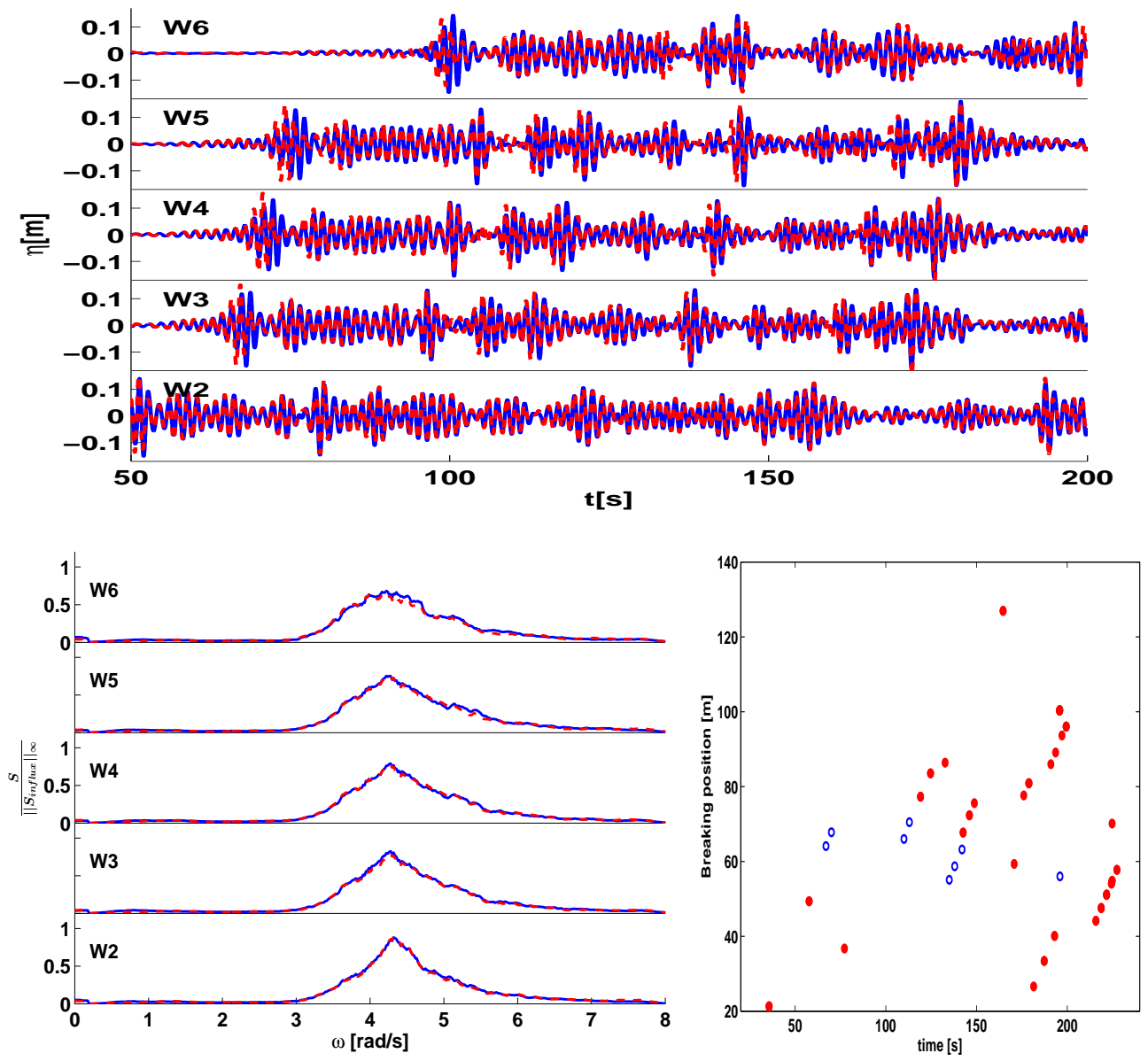

Figure A.22: Same as Fig. A.4 now for TUD1403Ir1 case.

Table A.22: Same as Table A.4 Now for TUD1403Ir1 case.

\begin{tabular}{|c|c|c|c|c|c|c|c|c|}
\hline $\mathrm{Tp}$ & $\lambda_{p}$ & $k_{p} \cdot a$ & $\mathrm{~W} 2$ & $\mathrm{~W} 3$ & $\mathrm{~W} 4$ & $\mathrm{~W} 5$ & W6 & Crel \\
\hline 1.4 & 3.14 & 0.25 & 0.95 & 0.92 & 0.91 & 0.88 & 0.89 & 7.5 \\
\hline
\end{tabular}


Irregular wave: TUD1403Ir2
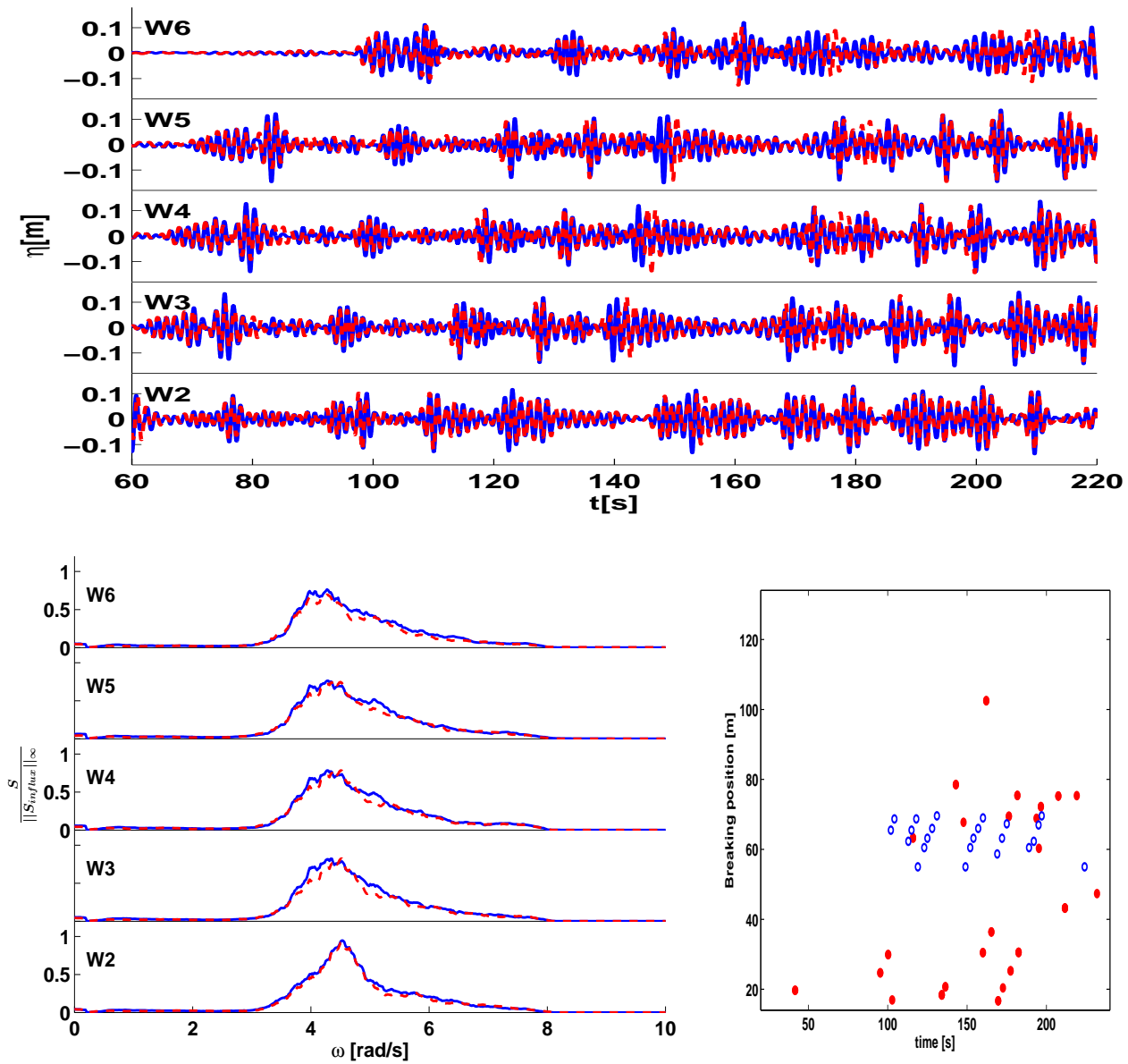

Figure A.23: Same as Fig. A.4 now for TUD1403Ir2 case.

Table A.23: Same as Table A.4 Now for TUD1403Ir2 case.

\begin{tabular}{|c|c|c|c|c|c|c|c|c|}
\hline $\mathrm{Tp}$ & $\lambda_{p}$ & $k_{p} \cdot a$ & $\mathrm{~W} 2$ & $\mathrm{~W} 3$ & $\mathrm{~W} 4$ & $\mathrm{~W} 5$ & $\mathrm{~W} 6$ & Crel \\
\hline 1.32 & 2.74 & 0.28 & 0.91 & 0.82 & 0.80 & 0.79 & 0.66 & 11 \\
\hline
\end{tabular}


Irregular wave: TUD1403Ir3
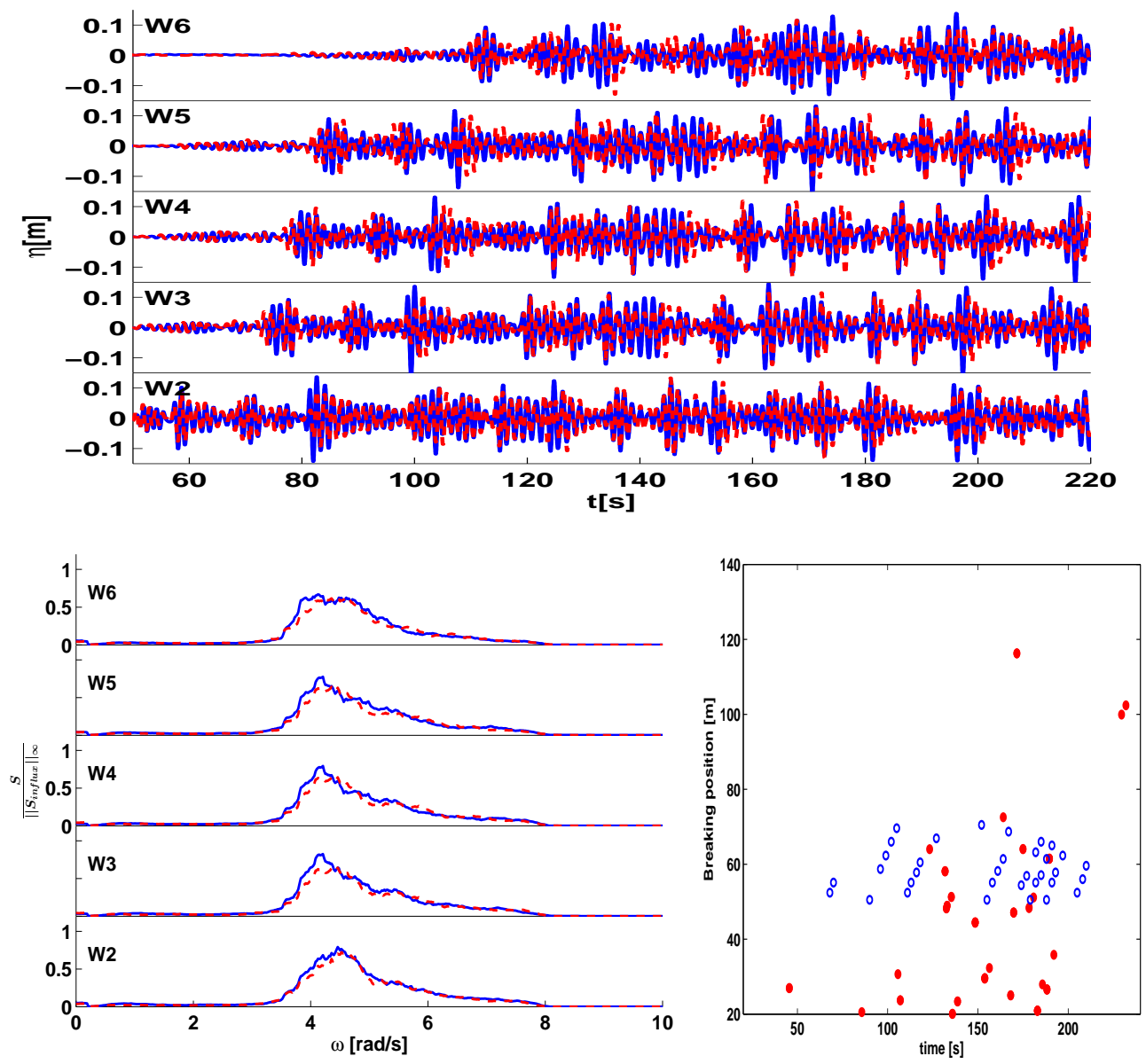

Figure A.24: Same as Fig. A.4 now for TUD1403Ir3 case.

Table A.24: Same as Table A.4 Now for TUD1403Ir3 case.

\begin{tabular}{|c|c|c|c|c|c|c|c|c|}
\hline $\mathrm{Tp}$ & $\lambda_{p}$ & $k_{p} \cdot a$ & $\mathrm{~W} 2$ & $\mathrm{~W} 3$ & $\mathrm{~W} 4$ & $\mathrm{~W} 5$ & $\mathrm{~W} 6$ & Crel \\
\hline 1.28 & 2.57 & 0.34 & 0.77 & 0.61 & 0.61 & 0.58 & 0.53 & 8.1 \\
\hline
\end{tabular}


Irregular wave: TUD1403Ir4
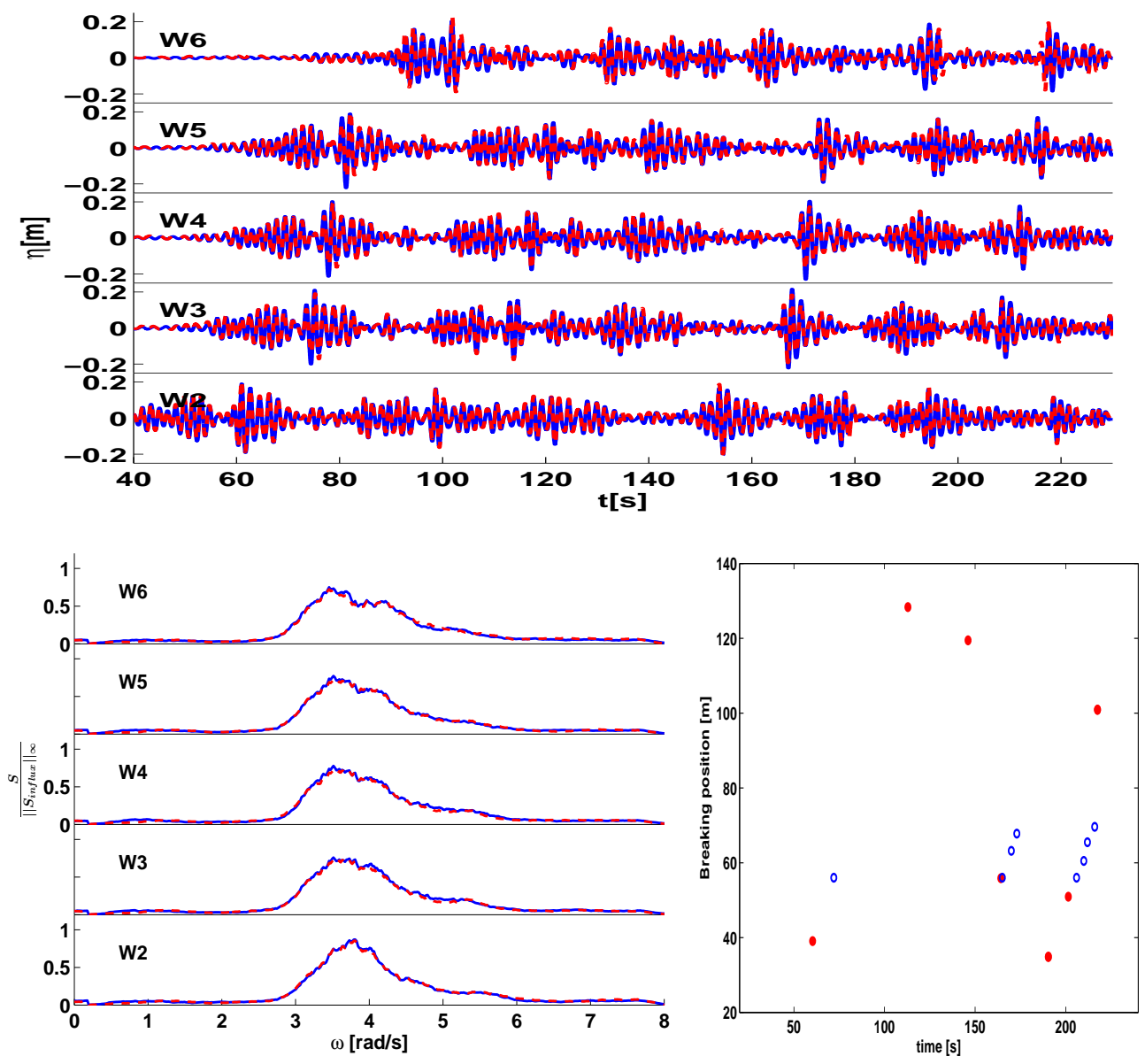

Figure A.25: Same as Fig. A.4 now for TUD1403Ir4 case.

Table A.25: Same as Table A.4 Now for TUD1403Ir4 case.

\begin{tabular}{|c|c|c|c|c|c|c|c|c|}
\hline $\mathrm{Tp}$ & $\lambda_{p}$ & $k_{p} \cdot a$ & $\mathrm{~W} 2$ & $\mathrm{~W} 3$ & $\mathrm{~W} 4$ & $\mathrm{~W} 5$ & $\mathrm{~W} 6$ & Crel \\
\hline 1.63 & 4.18 & 0.27 & 0.97 & 0.96 & 0.96 & 0.96 & 0.92 & 1.2 \\
\hline
\end{tabular}


Irregular wave: TUD1403Ir7 (non breaking)

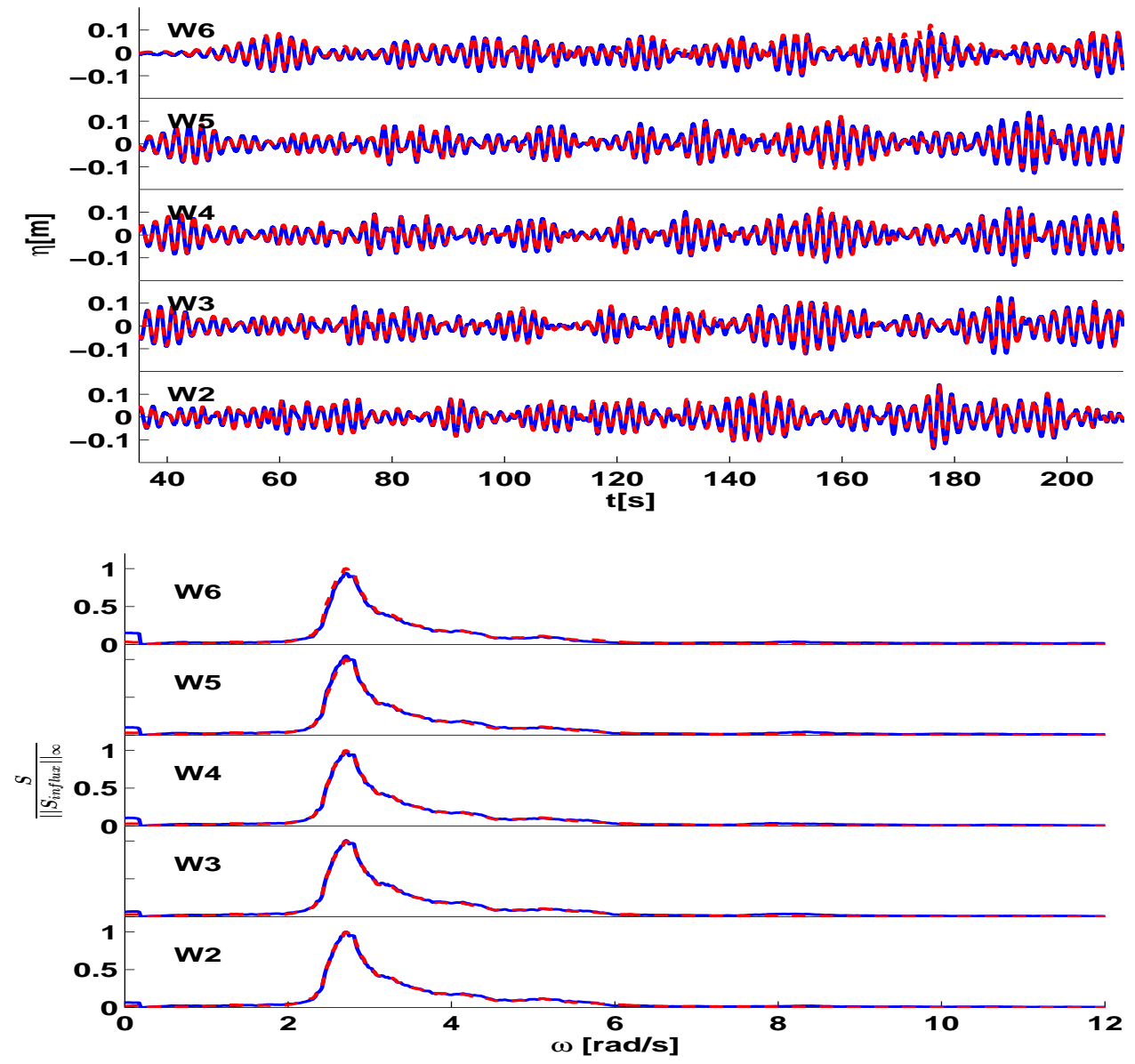

Figure A.26: Same as Fig. A.4 now for TUD1403Ir'7 case.

Table A.26: Same as Table A.4 Now for TUD1403Ir7 case.

\begin{tabular}{|c|c|c|c|c|c|c|c|c|}
\hline $\mathrm{Tp}$ & $\lambda_{p}$ & $k_{p} \cdot a$ & $\mathrm{~W} 2$ & $\mathrm{~W} 3$ & $\mathrm{~W} 4$ & $\mathrm{~W} 5$ & W6 & Crel \\
\hline 2.6 & 9.4 & 0.15 & 0.96 & 0.97 & 0.96 & 0.96 & 0.93 & 0.54 \\
\hline
\end{tabular}


Irregular wave: TUD1403Ir8
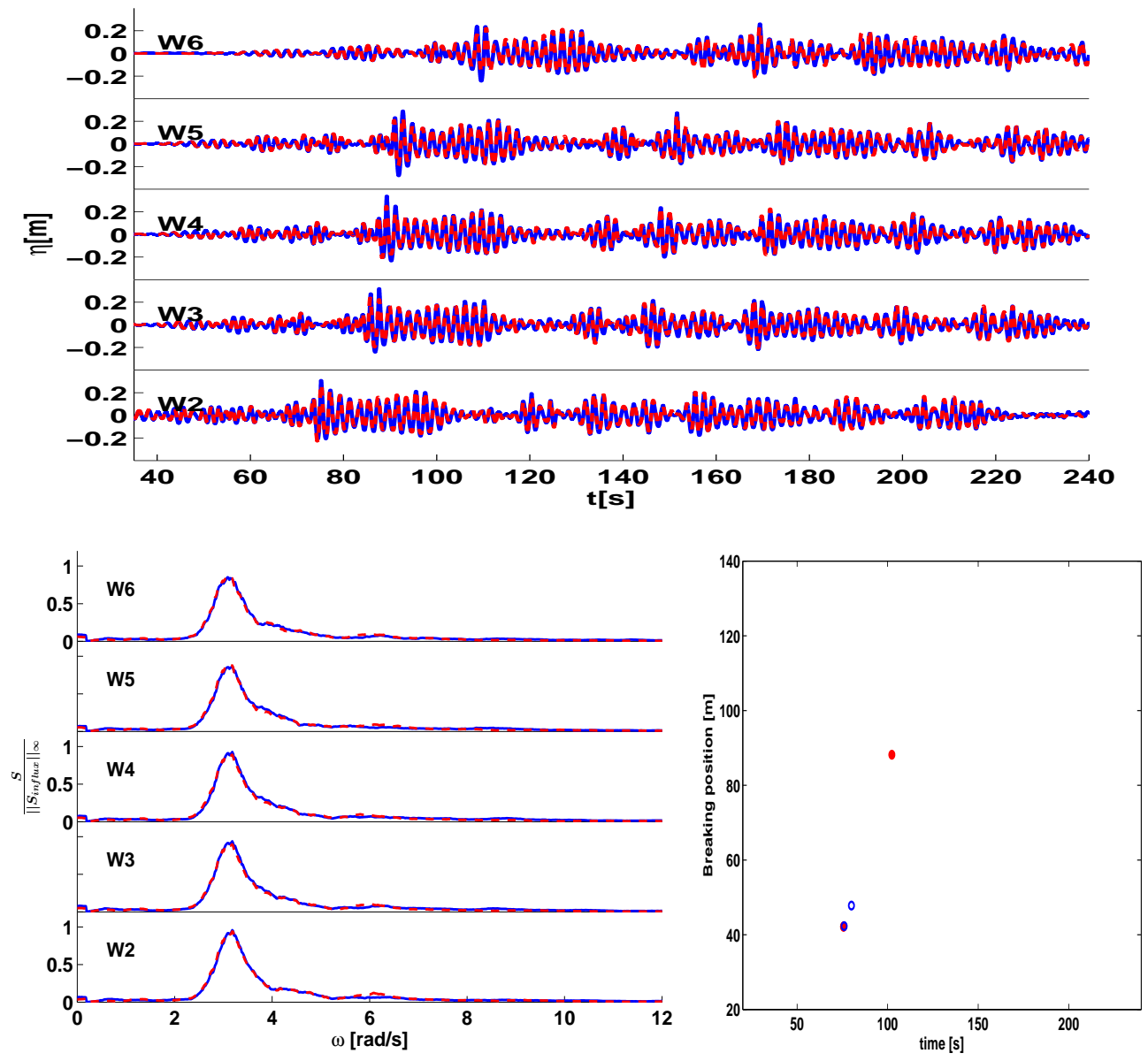

Figure A.27: Same as Fig. A.4 now for TUD1403Ir8 case.

Table A.27: Same as Table A.4 Now for TUD1403Ir8 case.

\begin{tabular}{|c|c|c|c|c|c|c|c|c|}
\hline $\mathrm{Tp}$ & $\lambda_{p}$ & $k_{p} \cdot a$ & $\mathrm{~W} 2$ & $\mathrm{~W} 3$ & $\mathrm{~W} 4$ & $\mathrm{~W} 5$ & $\mathrm{~W} 6$ & Crel \\
\hline 1.96 & 5.87 & 0.24 & 0.97 & 0.97 & 0.97 & 0.96 & 0.95 & 1.7 \\
\hline
\end{tabular}


Irregular wave: TUD1403Ir9
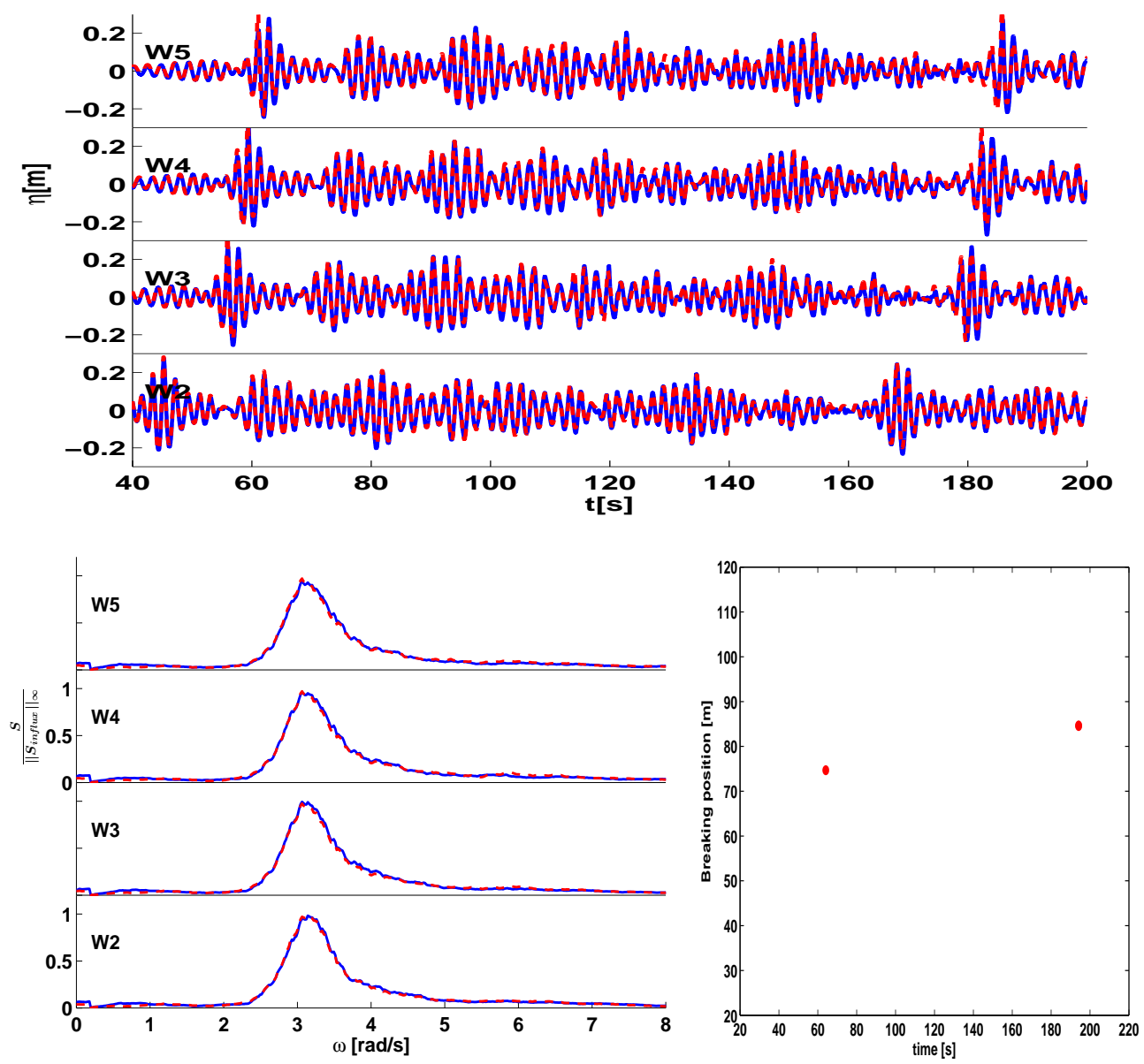

Figure A.28: Same as Fig. A.4 now for TUD1403Ir9 case. Info about breaking in experiment missing.

Table A.28: Same as Table A.4 Now for TUD1403Ir9 case.

\begin{tabular}{|c|c|c|c|c|c|c|c|}
\hline $\mathrm{Tp}$ & $\lambda_{p}$ & $k_{p} \cdot a$ & $\mathrm{~W} 2$ & $\mathrm{~W} 3$ & $\mathrm{~W} 4$ & W5 & Crel \\
\hline 1.96 & 5.87 & 0.24 & 0.98 & 0.97 & 0.96 & 0.96 & 1.87 \\
\hline
\end{tabular}


Irregular wave: TUD1403Ir10
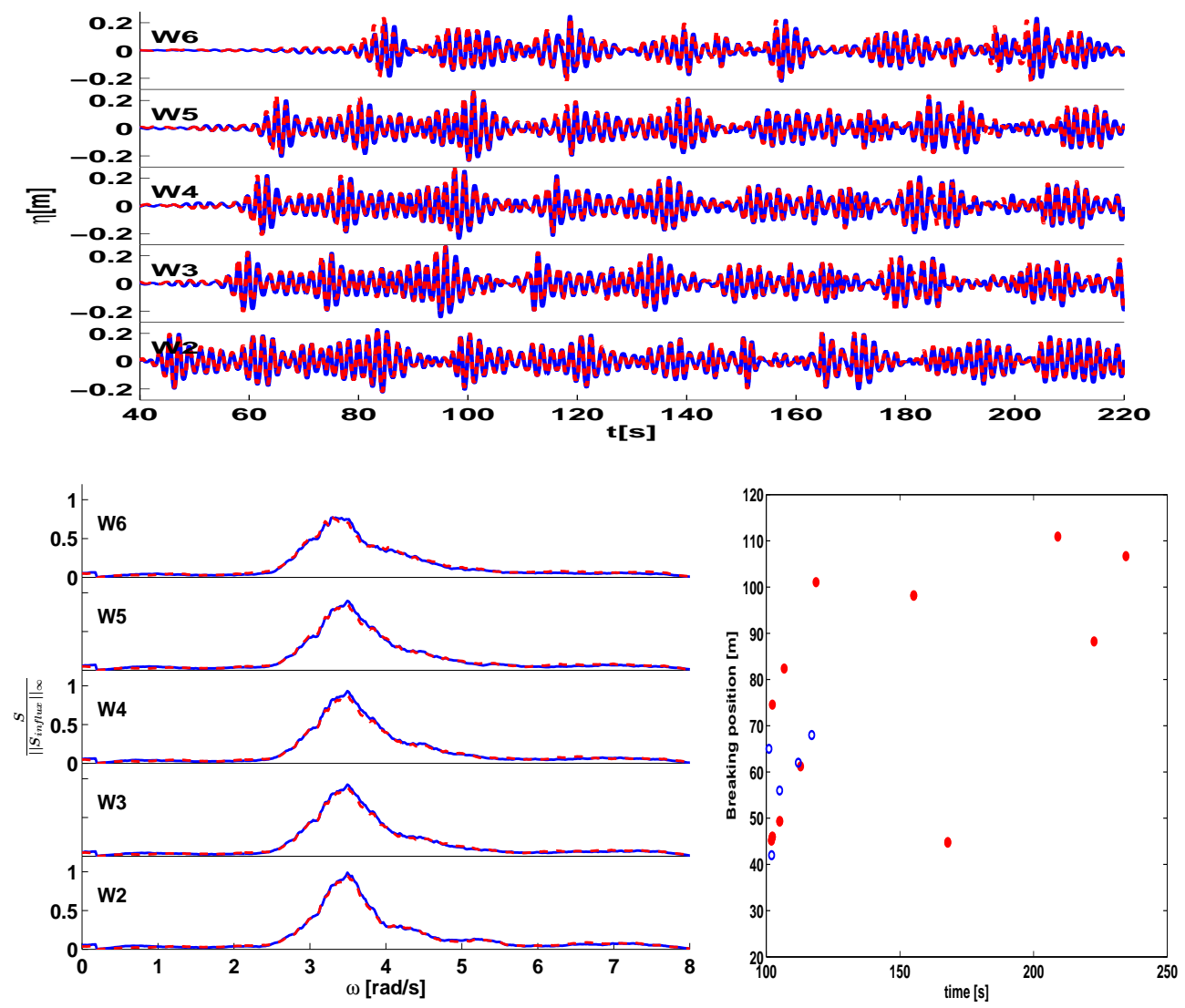

Figure A.29: Same as Fig. A.4, now for TUD1403Ir10 case.

Table A.29: Same as Table A.4 Now for TUD1403Ir10 case.

\begin{tabular}{|c|c|c|c|c|c|c|c|c|}
\hline $\mathrm{Tp}$ & $\lambda_{p}$ & $k_{p} \cdot a$ & $\mathrm{~W} 2$ & $\mathrm{~W} 3$ & $\mathrm{~W} 4$ & $\mathrm{~W} 5$ & W6 & Crel \\
\hline 1.8 & 5.02 & 0.27 & 0.97 & 0.96 & 0.95 & 0.94 & 0.86 & 1.11 \\
\hline
\end{tabular}


Irregular wave: TUD1403Ir11
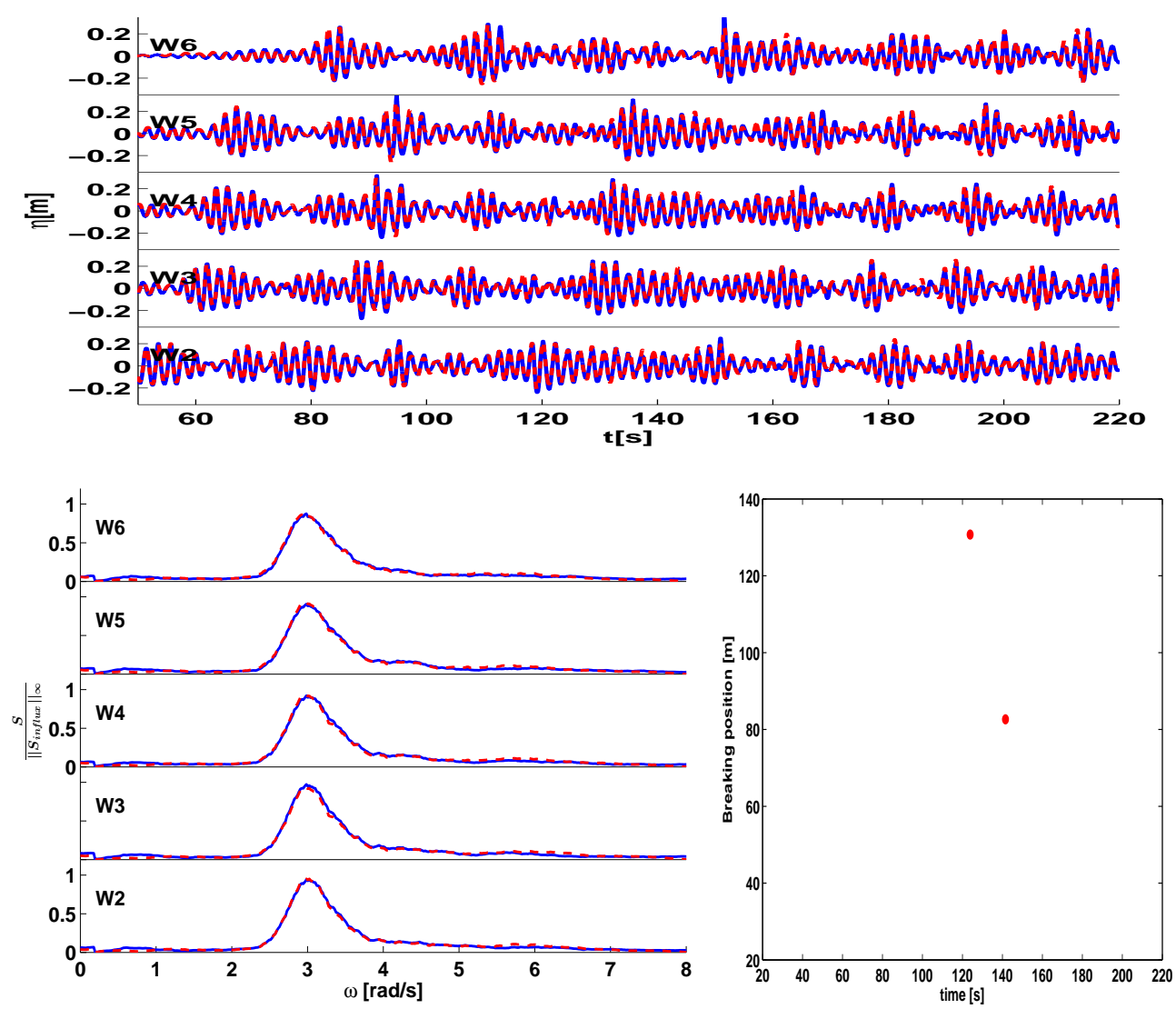

Figure A.30: Same as Fig. A.4 now for TUD1403Ir11 case. Info about breaking in experiment missing.

Table A.30: Same as Table A.4 Now for TUD1403Ir11 case.

\begin{tabular}{|c|c|c|c|c|c|c|c|c|}
\hline $\mathrm{Tp}$ & $\lambda_{p}$ & $k_{p} \cdot a$ & $\mathrm{~W} 2$ & $\mathrm{~W} 3$ & $\mathrm{~W} 4$ & $\mathrm{~W} 5$ & $\mathrm{~W} 6$ & Crel \\
\hline 2.08 & 6.55 & 0.23 & 0.97 & 0.96 & 0.96 & 0.95 & 0.94 & 1.8 \\
\hline
\end{tabular}


Soliton on Finite Background: TUD1403SFB1
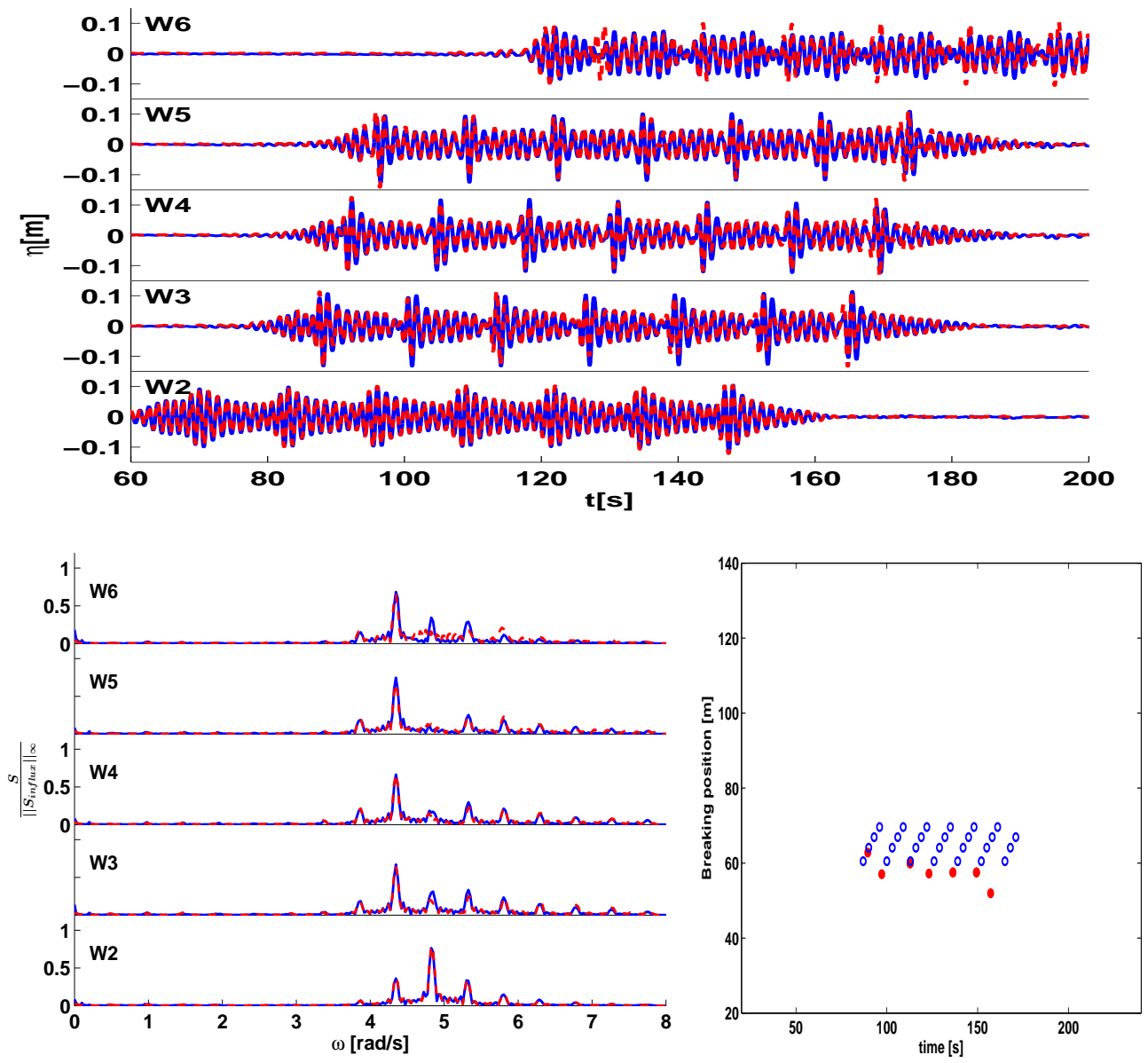

Figure A.31: Same as Fig. A.4 now for TUD1403SFB1 case.

Table A.31: Same as Table A.4 Now for TUD1403SFB1 case.

\begin{tabular}{|c|c|c|c|c|c|c|c|c|}
\hline $\mathrm{Tp}$ & $\lambda_{p}$ & $k_{p} \cdot a$ & $\mathrm{~W} 2$ & $\mathrm{~W} 3$ & $\mathrm{~W} 4$ & $\mathrm{~W} 5$ & W6 & Crel \\
\hline 1.3 & 2.65 & 0.17 & 0.98 & 0.88 & 0.89 & 0.89 & 0.77 & 1.9 \\
\hline
\end{tabular}


Soliton on Finite Background: TUD1403SFB2
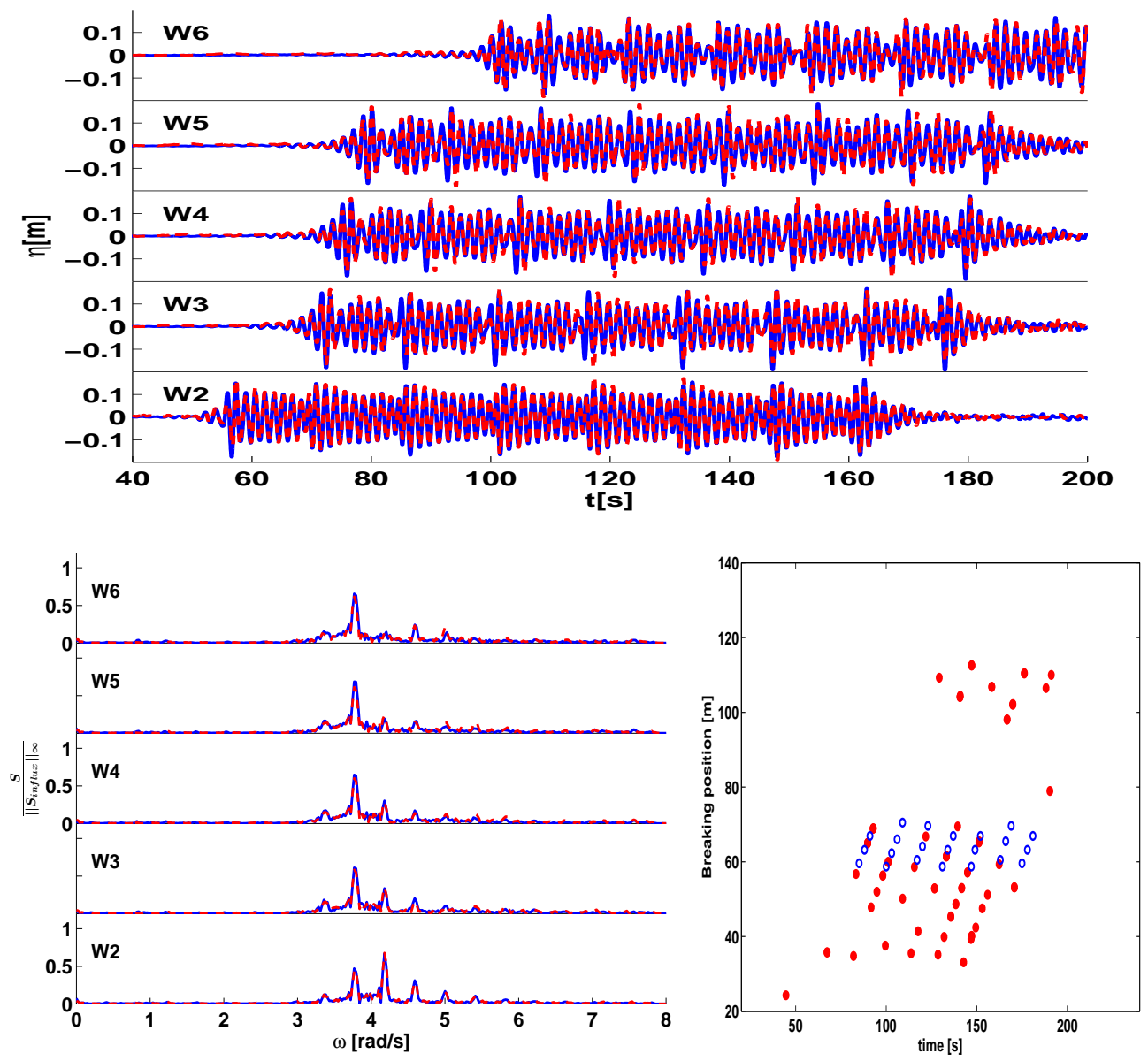

Figure A.32: Same as Fig. A.4 now for TUD1403SFB2 case.

Table A.32: Same as Table A.4. Now for TUD1403SFB2 case.

\begin{tabular}{|c|c|c|c|c|c|c|c|c|}
\hline $\mathrm{Tp}$ & $\lambda_{p}$ & $k_{p} \cdot a$ & $\mathrm{~W} 2$ & $\mathrm{~W} 3$ & $\mathrm{~W} 4$ & $\mathrm{~W} 5$ & W6 & Crel \\
\hline 1.5 & 3.53 & 0.26 & 0.97 & 0.91 & 0.91 & 0.89 & 0.88 & 3.2 \\
\hline
\end{tabular}


Harmonic focussing: TUD1403HF2 (non breaking)

-0.1.

- 0.1 .

E

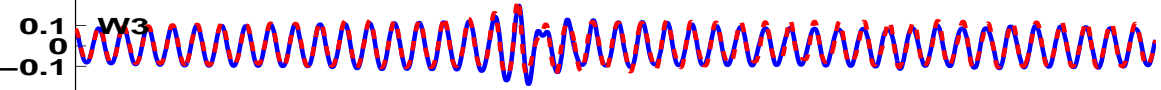

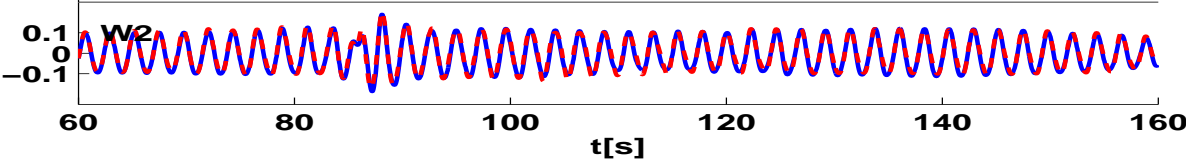

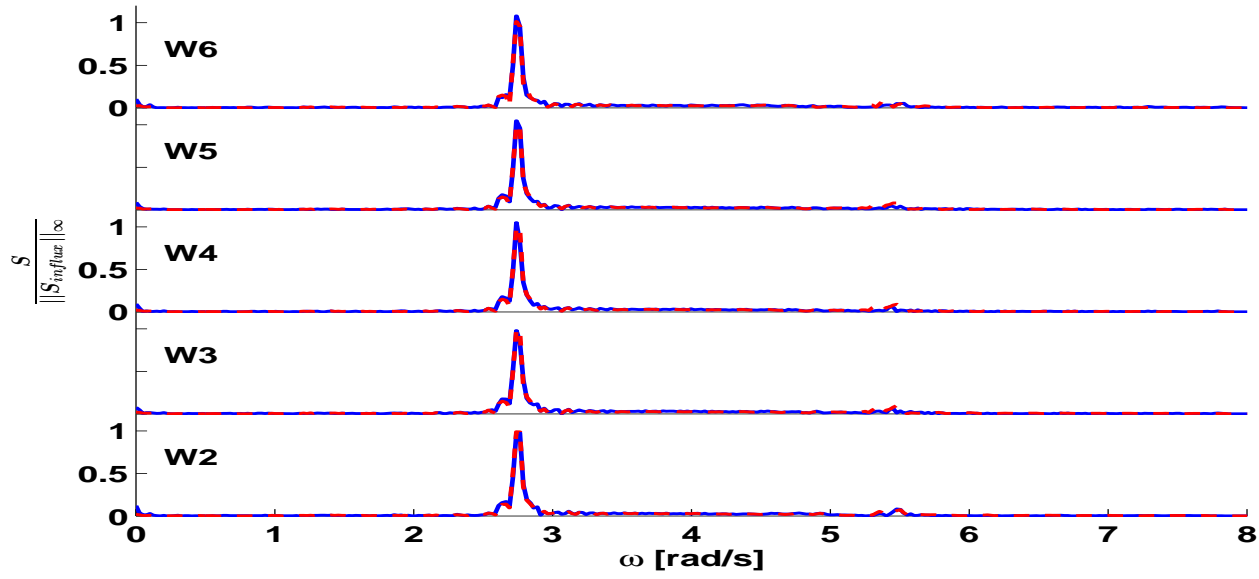

Figure A.33: Same as Fig. A.4 now for TUD1403HF2 case.

Table A.33: Same as Table A.4. Now for TUD1403HF2 case.

\begin{tabular}{|c|c|c|c|c|c|c|c|c|}
\hline $\mathrm{Tp}$ & $\lambda_{p}$ & $k_{p} \cdot a$ & $\mathrm{~W} 2$ & $\mathrm{~W} 3$ & $\mathrm{~W} 4$ & W5 & W6 & Crel \\
\hline 2.29 & 7.7 & 0.14 & 0.99 & 0.97 & 0.97 & 0.97 & 0.97 & 2.2 \\
\hline
\end{tabular}





\section{Bibliography}

T. A. A. Adcock, P. H. Taylor, S. Yan, Q. W. Ma, and P. A. E. M. Janssen. Did the draupner wave occur in a crossing sea? Proceedings of the Royal Society of London A: Mathematical, Physical and Engineering Sciences, 467(2134):3004-3021, 2011.

D. Adytia. Coastal zone simulations with variations Boussinesq modelling. $\mathrm{PhD}$ thesis, University of Twente, Enschede, May 2012.

D. Adytia and E. van Groesen. Optimized variational 1d boussinesq modelling of coastal waves propagating over a slope. Coastal Engineering, 64(0):139 - 150, 2012 .

M. L. Banner and W. L. Peirson. Wave breaking onset and strength for twodimensional deep-water wave groups. J. Fluid Mech., 585:93-115, 72007.

M. L. Banner and D. H. Peregrine. Wave breaking in deep water. Annu. Rev. Fluid Mech., 25(1):373-397, 1993.

H. Bateman. Notes on a differential equation which occurs in the two-dimensional motion of a compressible fluid and the associated variational problems. Proceedings of the Royal Society of London A: Mathematical, Physical and Engineering Sciences, 125(799):598-618, 1929.

S. Beji and J.A. Battjes. Experimental investigation of wave propagation over a bar. Coastal Engineering, 19(1-2):151 - 162, 1993.

S. Beji and J.A. Battjes. Numerical simulation of nonlinear wave propagation over a bar. Coastal Engineering, 23(1-2):1 - 16, 1994.

T. B. Benjamin, J. L. Bona, and J. J. Mahony. Model equations for long waves in nonlinear dispersive systems. Philosophical Transactions of the Royal Society of London A: Mathematical, Physical and Engineering Sciences, 272(1220):47-78, 1972 .

J. Boussinesq. Théorie des ondes et des remous qui se propagent le long d'un canal rectangulaire horizontal, en communiquant au liquide contenu dans ce canal des 
vitesses sensiblement pareilles de la surface au fond. J. Math. Pures Appl., 17(2): 55-108, 1872.

L. J. F. Broer. On the Hamiltonian theory of surface waves. Appl. Sci. Res., 29(1): 430-446, 1974.

B. Brunone and G. Tomasicchio. Wave kinematics at steep slopes: Second-order model. Journal of Waterway, Port, Coastal, and Ocean Engineering, 123(5):223$232,1997$.

O. Bühler and T. E. Jacobson. Wave-driven currents and vortex dynamics on barred beaches. Journal of Fluid Mechanics, 449:313-339, 122001.

T. Bunnik and E. van Groesen. Simulation of focused waves and impacts in a numerical wave basin. Proceeding of ICHD International Conference on Hydrodynamics, 2008.

R. Camassa and D. D. Holm. An integrable shallow water equation with peaked solitons. Phys. Rev. Lett., 71:1661-1664, Sep 1993.

J. H. E. Cartwright and H. Nakamura. What kind of a wave is hokusai's great wave off kanagawa? Notes and Records of the Royal Society, 63(2):119-135, 2009.

K. An Chang and P. L. F. Liu. Velocity, acceleration and vorticity under a breaking wave. Phys. Fluids, 10(1):327-329, 1998.

D. T. Cox and N. Kobayashi. Identification of intense, intermittent coherent motions under shoaling and breaking waves. Journal of Geophysical Research: Oceans, 105 (C6):14223-14236, 2000.

W. Craig and C. Sulem. Numerical simulation of gravity waves. J. Comput. Phys., 108(1):73-83, 1993.

A. D. D. Craik. The origins of water wave theory. Annual Review of Fluid Mechanics, $36(1): 1-28,2004$.

F. D'Alessandro and G. R. Tomasicchio. The BCI criterion for the initiation of breaking process in Boussinesq-type equations wave models. Coast. Eng., 55(12): $1174-1184,2008$.

J. C. Doering and M. A. Donelan. Acoustic measurements of the velocity field beneath shoaling and breaking waves. Coastal Engineering, 32(4):321 - 330, 1997.

J. M. Dudley, V. Sarano, and F. Dias. On hokusai's great wave off kanagawa: localization, linearity and a rogue wave in sub-antarctic waters. Notes and Records of the Royal Society, 67(2):159-164, 2013.

K. B. Dysthe. Note on a modification to the nonlinear schrodinger equation for application to deep water waves. Proceedings of the Royal Society of London A: Mathematical, Physical and Engineering Sciences, 369(1736):105-114, 1979. 
A. E. Green and P. M. Naghdi. A derivation of equations for wave propagation in water of variable depth. Journal of Fluid Mechanics, 78:237-246, 111976.

K. L. Heitner and G. W. Housner. Numerical model for tsunami run-up. J. Waterw. Harbors Coast. Eng. Div., 96(3):701-719, 1970.

Y. Kawata, Y. Tsuji, Y. Sugimoto, H. Hayashi, H. Matsutomi, Y. Okamura, I. Hayashi, H. Kayane, Y. Tanioka, K. Fujima, F. Imamura, M. Matsuyama, T. Takahashi, N. Maki, and S. Koshimura. Comprehensive analysis of the damage and its impact on coastal zones by the 2004 Indian Ocean tsunami disaster. 2005. URL www .tsunami .civil.tohoku .ac.jp/sumatra2004/report.html.

A. B. Kennedy, Q. Chen, J. T. Kirby, and R. A. Dalrymple. Boussinesq modeling of wave transformation, breaking, and runup. I: 1D. J. Waterw. Port Coast. Ocean Eng., 126(1):39-47, 2000.

O. Kimmoun and H. Branger. A particle image velocimetry investigation on laboratory surf-zone breaking waves over a sloping beach. J. Fluid Mech., 588:353-397, 2007.

G. Klopman, E. van Groesen, and M. W. Dingemans. A variational approach to boussinesq modelling of fully nonlinear water waves. Journal of Fluid Mechanics, 657:36-63, 82010.

D. J. Korteweg and G. de Vries. On the change of form of long waves advancing in a rectangular canal, and on a new type of long stationary waves. Philos. Mag., 39 (240):422-443, 1895 .

R. Kurnia and E. van Groesen. High order Hamiltonian water wave models with wave-breaking mechanism. Coastal Engineering, 93(0):55 - 70, 2014a.

R. Kurnia and E. van Groesen. Spatial-spectral Hamiltonian Boussinesq wave simulations. In Proceedings of International Conference on Computational and Experimental Marine Hydrodynamics (MARHY) Chennai, India, volume 2, pages 19-24, 2014b.

R. Kurnia and E. van Groesen. Design of wave breaking experiments and a-posteriori simulations. Memorandum 2042, Department of Applied Mathematics, University of Twente, Enschede, 2015a.

R. Kurnia and E. van Groesen. Localization in spatial-spectral methods for water wave applications. In Lecture Notes in Computational Science and Engineering (Proceedings of ICOSAHOM 2014, Salt Lake City, USA). Springer, 2015b.

R. Kurnia and E. van Groesen. Localization for spatial-spectral implementations of 1D Analytic Boussinesq equations. Submitted for publication, 2015c.

R. Kurnia, T. van den Munckhov, C. P. Poot, P. Naaijen, R. H. M. Huijsmans, and E. van Groesen. Simulations for design and reconstruction of breaking waves in a wavetank. In ASME 34th International Conference on Ocean, Offshore and Arctic Engineering (OMAE), St John's, NL, Canada. ASME, 2015. OMAE2015-41633. 
I. Lakhturov, D. Adytia, and E. van Groesen. Optimized Variational 1D Boussinesq Modelling for broad-band waves over flat bottom. Wave Motion, 49(2):309 - 322, 2012 .

A. L. Latifah and E. van Groesen. Coherence and predictability of extreme events in irregular waves. Nonlinear Processes in Geophysics, 19(2):199-213, 2012.

S. L. Lie. Mathematical modelling of generation and forward propagation of dispersive waves. PhD thesis, University of Twente, Enschede, May 2013.

S. L. Lie and E. van Groesen. Variational derivation of improved KP-type of equations. Phys. Lett. A, 374(3):411-415, 2010.

S. L. Lie, D. Adytia, and E. van Groesen. Embedded wave generation for dispersive surface wave models. Ocean Eng., 80(0):73 - 83, 2014.

J. C. Luke. A variational principle for a fluid with a free surface. J. Fluid Mech., 27:395-397, 11967.

P. J. Lynett, Tso-Ren Wu, and P. L.-F. Liu. Modeling wave runup with depthintegrated equations. Coastal Engineering, 46(2):89 - 107, 2002.

P. A. Madsen, O. R. Sørensen, and H. A. Schäffer. Surf zone dynamics simulated by a Boussinesq type model. Part I. Model description and cross-shore motion of regular waves. Coast. Eng., 32(4):255 - 287, 1997.

W. K. Melville and P. Matusov. Distribution of breaking waves at the ocean surface. Nature, 417(6884):58-63, 2002.

J. W. Miles. On Hamilton's principle for surface waves. J. Fluid Mech., 83(1): 153-158, 1977.

K. Mimura, N.and Yasuhara, S. Kawagoe, H. Yokoki, and S. Kazama. Damage from the great east japan earthquake and tsunami - a quick report. Mitigation and Adaptation Strategies for Global Change, 16(7):803-818, 2011.

I. Nikolkina and I. Didenkulova. Rogue waves in 20062010. Natural Hazards and Earth System Science, 11(11):2913-2924, 2011.

T. Okamoto and D. R. Basco. The Relative Trough Froude Number for initiation of wave breaking: Theory, experiments and numerical model confirmation. Coast. Eng., 53(8):675 - 690, 2006.

R. Paris, F. Lavigne, P. Wassmer, and J. Sartohadi. Coastal sedimentation associated with the December 26, 2004 tsunami in Lhok Nga, west Banda Aceh (Sumatra, Indonesia). Marine Geology, 238(14):93 - 106, 2007.

D. H. Peregrine. Water waves, nonlinear schrdinger equations and their solutions. The Journal of the Australian Mathematical Society. Series B. Applied Mathematics, 25:16-43, 71983. 
M. Perlin, J. He, and L. P. Bernal. An experimental study of deep water plunging breakers. Phys. Fluids, 8(9):2365-2374, 1996.

M. Perlin, W. Choi, and Z. Tian. Breaking waves in deep and intermediate waters. Annual Review of Fluid Mechanics, 45(1):115-145, 2013.

B. Riemann. Theorie der Abel'schen Functionen. Journal für die Reine und Angewandte Mathematik, 54:115-155, 1857.

H. A. Schäffer, P. A. Madsen, and R. Deigaard. A Boussinesq model for wave breaking in shallow water. Coast. Eng., 20(3-4):185 - 202, 1993.

F. Serre. Contribution à l'étude des écoulements permanents et variables dans les canaux. La Houille Blanche, 8:374-388, 1953.

L. Shemer. On kinematics of very steep waves. Natural Hazards and Earth System Science, 13(8):2101-2107, 2013.

P. Stansell and C. MacFarlane. Experimental investigation of wave breaking criteria based on wave phase speeds. J. Phys. Oceanogr., 32(5):1269-1283, 2002.

G. G. Stokes. On the theory of oscillatory waves. Transactions of the Cambridge Philosophical Society, 8:441-455, 1847.

I. A. Svendsen, K. Yu, and J. Veeramony. A Boussinesq breaking wave model with vorticity. Proc. 25th Int. Conf. Coastal Eng., ASCE, NewYork, pages 1192-1204., 1996.

Z. Tian, M. Perlin, and W. Choi. Energy dissipation in two-dimensional unsteady plunging breakers and an eddy viscosity model. J. Fluid Mech., 655:217-257, 2010.

F. C. K. Ting and J. T. Kirby. Observation of undertow and turbulence in a laboratory surf zone. Coastal Engineering, 24(1-2):51-80, 1994.

M. Tissier, P. Bonneton, F. Marche, F. Chazel, and D. Lannes. A new approach to handle wave breaking in fully non-linear Boussinesq models. Coastal Engineering, 67(0):54-66, 2012.

G. R. Tomasicchio. Capabilities and limits for ADVP measurements of breaking waves and bores. Coastal Engineering, 53(1):27 - 37, 2006.

E. van Groesen and Andonowati. Variational derivation of KdV-type models for surface water waves. Phys. Lett. A, 366(3):195 - 201, 2007.

E. van Groesen and Andonowati. Fully dispersive dynamic models for surface water waves above varying bottom, Part 1: Model equations. Wave Motion, 48(7):658 $-667,2011$.

E. van Groesen and Andonowati. Hamiltonian Boussinesq formulation of Wave-Ship interactions, Part 1: Evolution equations. Submitted, 2015. 
E. van Groesen and I. van der Kroon. Fully dispersive dynamic models for surface water waves above varying bottom, Part 2: Hybrid spatial-spectral implementations. Wave Motion, 49(1):198 - 211, 2012.

E. van Groesen, Andonowati, S. L. Lie, and I. Lakhturov. Accurate modelling of uni-directional surface waves. Journal of Computational and Applied Mathematics, 234(6):1747 - 1756, 2010.

J. Veeramony and I. A. Svendsen. The flow in surf-zone waves. Coast. Eng., 39(2-4): $93-122,2000$.

G. Wei, J. T. Kirby, S. T. Grilli, and R. Subramanya. A fully nonlinear Boussinesq model for surface waves. Part 1 . Highly nonlinear unsteady waves. Journal of Fluid Mechanics, 294:71-92, 71995.

R. Wilson. A rogue wave hits a tanker. NOAA photo library, 1993. URL www.photolib.noaa.gov/htmls/wea03621.htm

V. E. Zakharov. Stability of periodic waves of finite amplitude on the surface of a deep fluid. J. Appl. Mech. Tech. Phys., 9(2):190-194, 1968.

J. A. Zelt. The run-up of nonbreaking and breaking solitary waves. Coast. Eng., 15 (3):205-246, 1991. 


\section{Acknowledgments}

The research presented in this dissertation has been carried out over the past 4 years in the Applied Analysis (AA) group, Department of Applied Mathematics, University of Twente (UT). It is largely inspired and encouraged by my supervisor, family, friends and colleagues whom I would like to acknowledge.

First and foremost, I would like to express sincere gratitude to my supervisor, Prof. E. (Brenny) van Groesen, who I am indebted to for many things. I met Brenny for the first time when I applied an internship position at LabMath-Indonesia (LMI) in June 2011. At that time, i was finishing my master thesis. After I graduated, He offered me a position to pursue PhD degree at University of Twente and never thought before that the opportunity would bring me this far. I am thankful to him for the attentive guidance, fruitful discussions and the continuous support. His significant role helped me in finishing this dissertation. Again, I am grateful to him for giving me an opportunity to work as a post-doctoral researcher at UT and LMI starting from 1 February 2016.

I would like to pronounce my sincere thanks to Prof. Stephan van Gils, the chair of AA group, for the opportunity to work in his group, and also his willingness to be one of my graduation committee.

I would also like to thank other members of my graduation committee: Prof. Frederic Dias, Prof. Rene Huijsmans, Prof. Arthur Veldman, Prof. Bayu Jayawardhana, and Dr. Tim Bunnik for agreeing in the committee and for reviewing my thesis. I would also thank Prof. Peter Apers as the chairperson and the secretary of my graduation committee.

I would also like to express my gratitude to Prof. Rene Huijsmans for giving me an excellent opportunity to conduct the wave breaking experiments in a wave tank at Technical University of Delft. All support during the experiments from his group member: Peter Poot, Peter Naaijen and Toni van den Munckhof are greatly appreciated.

I thank MARIN for the use of the data, and in particular Dr. Tim Bunnik for discussions about the results and the organisation of the meetings.

I wish to express my thanks to Dr. Andonowati for providing me a nice research atmosphere during short visits at LMI. In LMI, I met many people with whom I can discuss about everything. I thank Didit, Andreas, Hafizh, Liam, Mourice, Meirita, 
Andy, Nugrahinggil, Abrari, Lia, Januar, Lawrance and others.

Many friends and colleagues in the Department of Applied Mathematics have shown a great help to my academic life at campus. A special word of thanks goes to the secretaries Marielle and Linda for all the administrative arrangements. I thank Gerard Jeurnink for involving me in educational activities. Thanks to everyone who I have shared my office with: Arnida, Anastasia, Elena, Devashish, Jurgen, Leonie, Freekjan. I would also like to thank the other members of the group: Huan, Lulu, Deepak, Edo, Bettina, Edson, Felix, Wilbert, Tatyana, and others.

I am really grateful for being surrounded by many warmhearted people during my stay in Enschede. I thank tante Soefiyati Hardjosumarto and Inggrid Proost for their kindness and for countless invitation to their house. I wish to express my thanks to Erwin vonk, Esther, Wisnu, Wenny, and Nida. I am also very thankful to all member of Indonesian Student Association in Enschede (PPIE) whom I cannot name one by one, which made my stay in Enschede enjoyable.

I am grateful to my family especially my sisters: Chiely, Inalia and Dian for their support. I am truly grateful to my parents, Handreas Syamdiputra and Herliana Taniman for their unconditional love, prayer and support. Finally, I thank my wife, Erika Tivarini for her love and support. Above all, I thank God for His grace and guidance in my life. 


\section{About the author}

Ruddy Kurnia was born on the $1^{\text {th }}$ of May 1987 in Bandung, Indonesia. He obtained a degree of Bachelor of Science from Physics department of Institut Teknologi Bandung (ITB), Indonesia in July 2009 on a subject of electromagnetic method for geophysics exploration.

In August 2009, he started his Master's study on double degree program of computational science at Kanazawa University, Japan and Institut Teknologi Bandung, Indonesia. He finished his study in August 2011, on a subject of particle methods for computational fluid dynamics.

From June 2011 to January 2012, he worked as an internship student at LabmathIndonesia on a subject of water wave modelling.

In February 2012, he started his Ph.D research in Applied Analysis group, Department of Applied Mathematics, University of Twente. After 4 years, he finished his doctoral study which the result of his research is presented in this dissertation.

Starting from 1 February 2016, he works as a post-doctoral researcher at University of Twente and Labmath-Indonesia. In this project, he is extending the applicability of the HAWASSI software. 